\title{
Satzbaupläne als Zeichen: die semantischen Rollen des Deutschen in Theorie und Praxis
}

\begin{abstract}
Thema des Beitrags sind Satzbaupläne als komplexe syntaktisch-semantische Sprachzeichen. An der Schnittstelle von Valenztheorie (GTA 2017; Ágel 2015, 2000) und Konstruktionsgrammatik (Goldberg 1995; Schneider 2014) werden Satzbaupläne nicht als formale Zeichen wie in traditionellen Satzbauplankonzepten, sondern als abstrakte Zeichen mit einer Formseite (Satzglieder) und einer Inhaltsseite (signifikativ-semantische Rollen) aufgefasst (GTA 2017; Höllein 2019). Die Satzbauplanzeichen sind dabei vollständig abstrakte Zeichen, die kein konkretes phonologisches oder morphologisches Material enthalten. Die Inhaltsseiten der Satzbauplanzeichen werden mithilfe signifikativ-semantischer Rollen modelliert. Die signifikative Semantik und die in diesem Theorierahmen gewonnenen semantischen Rollen unterscheiden sich radikal von existierenden Konzepten semantischer Rollen. Diese werden von Welke (2019, 2011, 2005, 1988), dem Begründer der signifikativen Semantik, als denotativ-semantische Rollen bezeichnet, weil sie die (außereinzelsprachliche) Bezeichnung zentrieren, während die signifikative Semantik die Bedeutung einzelsprachlich und prototypentheoretisch fokussiert. Im empirischen Teil des Beitrags werden alle Satzbauplanzeichen eines kompletten Zeitungsartikels ermittelt und die signifikativ-semantische Analyse einer traditionellen denotativ-semantischen gegenübergestellt. Somit hebt sich dieser Beitrag empirisch auch von denotativ-semantischen Beiträgen insofern ab, als die Überprüfung des Ansatzes nicht an einzelnen Beispielsätzen, sondern an einem ungekürzten und nichtvereinfachten Text erfolgt. Der theoretisch-empirische Ertrag des Beitrags ist einerseits die Aufstellung eines kompletten semantischen Rollensets, andererseits eine umfassende Beschreibung der Satzbaupläne des Gegenwartsdeutschen.
\end{abstract}

Anmerkung: Die bibliographischen Angaben des analysierten Zeitungstextes sind im Anhang zu finden.

Vilmos Ágel, Institut für Germanistik, Universität Kassel

Dagobert Höllein, Institut für Germanistik, Universität Kassel 


\section{Einleitung}

Ziel des Aufsatzes ist es zu zeigen, dass die überwiegende Mehrheit syntaktischer Grundstrukturen (Satzbaupläne) des Gegenwartsdeutschen sich auch semantisch interpretieren lässt und somit komplexe syntaktisch-semantische Sprachzeichen darstellt. Unter Letzteren verstehen wir die Zuordnung von grammatischen Werten (Satzgliedern) zu semantischen. ${ }^{1}$ Die Voraussetzung für diese Auffassung ist allerdings, dass die semantischen Werte, d.h. die Beschreibung der Inhaltsseite von Satzbauplänen, signifikativ-semantisch modelliert werden und nicht wie traditionell denotativ-semantisch. ${ }^{2}$ Signifikativ-semantische Rollen bekommen nämlich im Gegensatz zu den als universal gesetzten denotativ-semantischen ihren Wert (valeur) einzelsprachlich-systemintern.

Erstaunlicherweise hat es die denotative Semantik nicht geschafft, ein theoretisch abgesichertes Set von Rollen zu präsentieren, und das, obwohl die stipulierten Rollen nicht einmal an natürlich-sprachlichem Material eingeführt wurden. Hätte man versucht, die Rollen wenigstens an kurzen kohärenten Textsequenzen zu erproben, wären die Probleme dieser Rollen schnell offen zu Tage getreten.

Wir möchten im Folgenden einerseits zeigen, dass ein komplettes, kohärentes Set von (signifikativ-)semantischen Rollen, mit denen man auch größere Texte beschreiben kann, möglich ist. Dazu wird zuerst in Kap. 2 das System der gegenwartsdeutschen signifikativ-semantischen Rollen in Abgrenzung zu den denotativ-semantischen entwickelt (zu den Rollensets siehe Kap. 4.2). Verbindet man dieses System mit einem etablierten Set von grammatischen Satzbauplänen, erhält man die Satzbauplanzeichen der deutschen Gegenwartssprache (siehe Kap. 5.2). Diese stellen prototypisch organisierte wertbezogene Schemata dar.

Andererseits werden die Vorteile dieser neuen Auffassung im Rahmen einer empirischen Untersuchung demonstriert. Dazu wird im Beitrag eine denotativ-semantische und eine signifikativ-semantische Rollenanalyse des Die Zeit-

1 Die Architektur der Grammatik wird in der GTA (2017: 18-38) in Analogie zu der FunktionArgument-Wert-Formel von Allwood, Andersson \& Dahl (1973: 8-11) beschrieben. Daraus ergeben sich grammatische Werte auf drei Analyseebenen: Textglieder (Makroebene), Satzglieder (Mesoebene) und Wortgruppenglieder (Mikroebene). Um Missverständnissen vorzubeugen: Zeichen verfügen im Saussure'schen Sinn untrennbar über Ausdruck- und Inhaltsseite. Unter Zuordnung wird hier die Analyse und Verbindung beider Seiten verstanden.

2 Der Terminus denotativ-semantisch stammt von Fleischmann (1985) und Welke (2019, 1988), die damit die existierenden bezeichnungszentrierten, auf die außersprachliche Wirklichkeit zielenden Ansätze zu semantischen Rollen benennen, um sie vom neuen signifikativsemantischen, bedeutungszentrierten Ansatz abzugrenzen (siehe Kap. 2). 
Artikels Vorsicht, Brennpunkt! vorgenommen (siehe Kap. 4.3). ${ }^{3}$ In der Kontrastierung beider Ansätze zeigt sich, dass die signifikative Semantik in der Lage ist, ganze Texte semantisch adäquat und exhaustiv zu analysieren. Die Voraussetzung hierfür ist eine textbasierte Syntaxtheorie, mit der sich die grammatischen Grundstrukturen von/in Texten identifizieren lassen (Kap. 4.1). Gleichzeitig wird offenbar, dass die denotative Rollensemantik es in 50 Jahren nicht geschafft hat, ein Modell vorzulegen, mit dem man einen durchschnittlichen Zeitungstext lückenlos und widerspruchsfrei analysieren könnte. Dies hat mit vielen Faktoren (unterschiedlichen Gewichts) zu tun: mit der Auffassung, dass Grundstrukturen keine Zeichen seien, sondern dass Syntax und Semantik autonom - und als solche aufeinander zu beziehen - seien, mit der Verwechslung von Bedeutung und Bezeichnung, von Einzelsprache und Welt, mit der Nichtunterscheidung von aktiven und inaktiven Rollen, mit Ad hoc-Listenansätzen, die jederzeit ergänzbar, reduzierbar, revidierbar oder ersetzbar sind, mit der fehlenden Rekonstruktion eines Prädikatsbegriffs, mit dem ungeklärten Status des Prädikativs, mit der Konzentration auf Subjekt und Direktes Objekt und mit einer fehlenden Theorie von Präpositionalobjekten. ${ }^{4}$ Daraus ergeben sich die erwähnten Lücken und Widersprüche, die auftreten, sobald man versucht, mit einem denotativ-semantischen Modell zu arbeiten.

\section{Semantische Rollen: denotativ- oder signifikativ-semantisch?}

Das denotativ-semantische Mainstream-Modell semantischer Rollen entspricht trotz einiger Neuerungen wie dem Protorollenansatz von Dowty (1991) - nach wie vor maßgeblich dem 50 Jahre alten denotativ-semantischen Kasusrollenkonzept Fillmores (1968), und das obwohl seit den 70er Jahren die Schwächen der Konzeption bekannt sind (Helbig 1992: 24-25, 1979: 67-68). Die Kritik ist - mögli-

3 Der Zeitungsartikel samt bibliographischen Angaben ist im Anhang abgedruckt.

4 Ziem \& Lasch (2013: 118-119) und Lasch (2016: 32-33) erwähnen eine Aussage Fillmores, in der Fillmore die Suche nach einem fixen Rollenset als „gescheitert“ (Ziem \& Lasch 2013: 118) bzw. „problematisch“ (Lasch 2016: 32) ansehe und deshalb in Framenet auf ein offenes Rollenset setze. (In beiden Literaturverzeichnissen findet sich keine zur zitierten Seitenzahl passende Publikation Fillmores, mutmaßlich verweisen sie auf Fillmore (2006: 216).) Aus theoretischer Perspektive ist die Öffnung des Rollensets problematisch, da damit die Kernannahme der Rollensemantik, mit einem festen Set von Rollen syntaktische Strukturen inhaltlich zu beschreiben, aufgegeben wird. 
cherweise wegen des großen Erfolgs des Rollenkonzepts - weitgehend ungehört geblieben. Von den Vertretern der denotativen Semantik wird die ungewisse Gesamtanzahl der Rollen als Problem gesehen (Levin \& Rappaport Hovav 2008). Ein Problem, das man mit Dowtys Reduktion auf zwei prototypische Rollen weitgehend gelöst zu haben glaubt (Primus 2012, 1999). So richtig Dowtys Ansatz in Bezug auf die Prototypik u. E. auch ist, er löst nicht das Hauptproblem der denotativen Semantik, sondern verdeckt dieses vielmehr.

Aus signifikativ-semantischer Perspektive ist das Hauptproblem der denotativen Semantik, dass keine einzelsprachlichen Strukturen analysiert werden, sondern die Bezeichnung (das Denotat) (Fleischmann 1985; Starosta 1988, 1978; Welke 2019, 2011; GTA 2017; Höllein 2019). ${ }^{5}$ Denn die denotativ-semantische Konzeption zentriert explizit oder implizit die außersprachliche Wirklichkeit und weist von dieser ausgehend sprachlichen Strukturen semantische Rollen zu. Dies wird an folgenden Beispielen aus Levin \& Rappaport Hovav (2008: 28) bzw. aus Levin (1999: 224) deutlich, die hier in deutscher Übersetzung wiedergegeben werden:

(1) Der Ingenieur zerstört die Brücke. (PATIENT/CONSUMED OBJECT)

(2) Der Ingenieur bemalt die Brücke. (INCREMENTAL THEME)

(3) Der Ingenieur bewegt die Brücke. (THEME)

(4) Der Ingenieur baut die Brücke. (EFFECTED OBJECT/FACTITIVE)

(5) Der Ingenieur wäscht die Brücke. (LOCATION/SURFACE)

(6) Der Ingenieur überquert die Brücke. (РАтн)

(7) Der Ingenieur sieht die Brücke. (Stimulus/OBJect OF PERCEPTION)

Levin (1999: 224) weist den Akkusativobjekten in den Beispielsätzen (1)-(7) jeweils unterschiedliche denotativ-semantische Rollen zu. Der Grund ist, dass die Situationen (in der außersprachlichen Wirklichkeit) und nicht die einzelsprachlich entworfenen Sachverhalte analysiert werden. Levins im Original zwölf Beispiele (und zwölf unterschiedliche Rollen) umfassende Liste ist in Levin \& Rappaport Hovav (2008) mit dem Hinweis versehen, dass „even these examples do not capture the full semantic breadth of the notion ,object' in English“" (Levin \& Rappaport Hovav 2008: 28). Die Liste würde sogar - analysierte man denotativ-semantisch konsequent - unendlich länger, da es so viele Rollen geben müsste, wie es Situationen in der Welt gibt.

5 In Höllein (2019: 4-28) findet sich eine ausführliche Darstellung zur signifikativen Semantik. Dort werden - am Beispiel der Präpositionalobjekte - auch spezifischere Fragen wie die nach der Rollenhomonymie erläutert (Höllein 2019: 80-82). 
Überspitzt formuliert ist die denotative Semantik aus signifikativ-semantischer Perspektive überhaupt keine Semantik, sondern eine nicht sprachwissenschaftliche Sachkunde (Coseriu 1970: 4; Ullmann 1973: 74f.). Denn es werden bewusst oder unbewusst - nicht die Bedeutungen einzelsprachlicher Strukturen (Signifikate) analysiert, sondern die Bezeichnungen (Denotate). Ein Beispiel denotativer Semantik aus dem lexikalischen Bereich ${ }^{6}$ ist Löbners (2018: 194) „Bleistift-Frame“, der die Bedeutung beschreiben soll, aber weitgehend die Bezeichnung erfasst:

Ein „grüner Bleistift“ ist kein monochrom grünes Objekt. Wenn es sich um einen konventionellen Bleistift aus Holz mit einer üblichen Bleistiftmine handelt, sind weder das Holz noch die Mine grün, sondern das Holz ist grün lackiert. Wahrscheinlich hat der Bleistift auch noch Schrift, Firmensymbole etc. auf den Schaft aufgetragen; wir wollen das vernachlässigen. Das Prädikat „grün“ verlangt von seinem Argument, dass es als Ganzes grün oder nicht grün ist.

(Löbner 2018: 194)

Nur der letzte Satz erfasst die Bedeutung, der Rest ist eine Beschreibung der Bezeichnung. Löbner konstatiert zwar, dass der „Bleistift-Frame [...] Weltwissen ab[bildet]“ und „,[d]er lexikalische Bedeutungsframe [.. .] wesentlich schlanker [wäre]“ (Löbner 2018: 193), begründet diese Aussage aber wie folgt:

denn er [= der Bleistift-Frame VÁ/DH] müsste auf alle möglichen und historischen Erscheinungsformen von Bleistiften zutreffen, auf Bleistifte mit einem Holzschaft ebenso wie auf Bleistifte mit Fallminen und einem Kunststoff- oder Metallgehäuse, auf Bleistifte, die nur aus einer Mine bestehen, oder auf Bleistifte mit Minen aus anderem Material. Vermutlich sind nur der Bestandteil „Mine“ und der Affordanzkomplex als Bestandteile des Lexikonframes anzusehen.

(Löbner 2018: 193)

An dieser Begründung wird deutlich, dass Löbner in einer - nicht einmal prototypentheoretischen - Bezeichnungslehre verhaftet ist, die davon ausgeht, dass die Bedeutung eines Lexems dann richtig beschrieben ist, wenn sie alle vom Lexem bezeichneten Dinge in der außersprachlich gedachten Welt $\mathrm{zu}$ fassen in der Lage ist. Abgesehen davon, dass u. E. die Annahme einer außersprachlichen Wirklichkeit nicht zielführend - präziser: unmöglich - ist (Höllein 2019: 18), muss eine signifikativ-semantische Bedeutungsbeschreibung grundsätzlich anders gestaltet sein: Sie ist zunächst prototypensemantisch, da sie sich ganz im Sinne von Köpcke/Hinze (2011: 68) dazu eignet, „Bedeutungszusammenhänge zu modellieren“. Vor allem aber ist sie im Saussure'schen Sinn differentiell gefasst. D. h., die Bedeutung des Lexems Bleistift

6 In der denotativen Semantik finden sich prototypischerweise ,Bedeutungsanalysen“ auf Lexemebene und hier wiederum solche von Konkreta. 
ist ihre Differenz zu den Bedeutungen anderer Lexeme, mit denen es ein Wortfeld bildet. Jede positive Bedeutungsbeschreibung kann nur Hilfsmittel sein, nicht aber (semantische) Beschreibung. Saussure ahnt jedoch bereits die Schwierigkeiten, die die Semantik mit seinem Bedeutungsbegriff haben würde und behielt (leider) Recht:

Man wird das rein differentielle Wesen jedes der Elemente [. . .], denen wir [. . .] zuschreiben, daß sie existieren, nie ausreichend verinnerlicht haben: Es gibt in keiner Ordnung auch nur irgendeines, das diese unterstellte Existenz aufweist - wenn wir auch vielleicht, ich gestehe es ein, zugeben müssen, daß der Geist ohne diese Fiktion [. . . ] nicht im Stande wäre, eine derartige Menge von Differenzen zu beherrschen, wo es nirgends [. . . ] einen positiven und festen Anhaltspunkt gibt.

(Saussure 2003: 129-130)

Die signifikative Semantik analysiert deshalb Bedeutungen oder sprachliche Inhalte ausgehend von einzelsprachlichen Strukturen. Auf die Beispielreihe angewendet bedeutet das Folgendes: Die Akkusativobjekte in den Beispielsätzen (1) - (7) tragen signifikativ-semantisch dieselbe Rolle, da sie lexikalische Realisierungen derselben gegenwartsdeutschen syntaktischen Struktur sind und sich auf der Ebene signifikativ-semantischer Rollen sprachlich nicht voneinander unterscheiden. Alle sind Instanzen der semantischen Rolle HANDLUNGSGEGENSTAND. ${ }^{7}$

Handlungsgegenstand ist die Rolle, die Dowtys (1991) Proto-Patient entspricht. D. h. in Bezug auf das Analyseergebnis ist Dowtys Konzept - im konkreten Fall - identisch mit der signifikativ-semantischen Analyse. Der Weg zur identischen Analyse weicht allerdings stark ab: Signifikativ ist die Begründung der Analyse, dass die einzelsprachliche Leistung der Akkusativobjekte - ihre Bedeutung - so viel Ähnlichkeit aufweist, dass sie unter der gleichen Rolle subsumierbar sind. Dowtys zwar ebenfalls prototypischer, aber ansonsten denotativer Ansatz analysiert die Akkusativobjekte als PATIENS, weil die dem Satz (nach Dowtys Auffassung) zugrundeliegende Instanz in einer konkreten außersprachlichen Situation mit einer Instanz in einer prototypischen außersprachlichen Situation abgeglichen wird. D.h., auch der Dowty'schen Rollenanalyse liegt keine sprachliche Analyse, sondern lediglich ein Abgleich von konkreten außersprachlichen Situationen mit prototypischen zugrunde.

Formale Analyseprobleme entstehen bei Dowtys Konzept, sobald dreiwertige Sachverhalte analysiert werden sollen. Denn nach dem Theta-Kriterium (Chomsky 1993: 36) darf jede Rolle nur einmal pro Satz vergeben werden.

7 Die Beispiele (1)-(7) stellen also aus signifikativ-semantischer Sicht alle HANDLUNGen dar. In Kap. 4.2 werden sie aufgegriffen und signifikativ-semantisch eingeordnet. 
Dowty (1991) sieht nach allgemeiner Lesart aber nur zwei vor und kann deshalb Sätze wie (8) nicht (vollständig) analysieren:

(8) Ich gebe dir das Buch.

Dowty sieht dieses Problem selbst und begegnet ihm mit der „Corollary 2“, die sich für ihn aus seinem „Argument-Selection-Principle“ (Dowty 1991: 576) ergibt und die hier wörtlich zitiert werden soll:

With a three-place predicate, the nonsubject argument having the greater number of entailed Proto-Patient properties will be lexicalized as the direct object and the nonsubject argument having fewer entailed Proto-Patient properties will be lexicalized as an oblique or prepositional object (and if two nonsubject arguments have approximately equal numbers of entailed P-Patient properties, either or both may be lexicalized as direct object). （Dowty 1991: 576)

Wie man dem Zitat entnehmen kann, gibt es einen sehr deutlichen Unterschied zwischen Dowtys eigenem Konzept und dessen Standard-Verständnis. Denn Dowty weist den genannten Satzgliedern subject, direct object und oblique/prepositional object überhaupt keine semantischen Rollen zu. Vielmehr geht es ihm um die Emergenz dieser Satzglieder mithilfe von semantischen Rollen: Die Satzglieder bekommen ihre Satzgliedwerte erst über die Anzahl der ihnen zugeordneten Proto-AgEns/PATIENS-Merkmale. Dieser Ansatz wurde in der formalen Linguistik verständlicherweise ,übersehen‘. Hier wurde Dowty so verstanden, dass er über prototypische AGENS- und PATIENS-Merkmale semantische Rollen vergibt (Primus 1999: 36-47).

Unsere Kritik bleibt allerdings bestehen: Dowty muss entweder zwei Satzgliedern die gleiche Rolle zuweisen, was zu Rollensynonymie und einem Verstoß gegen das Theta-Kriterium führt; oder er kann das dritte Argument nicht analysieren, was angesichts vieler dreiwertiger Verben eine nicht $\mathrm{zu}$ unterschätzende theoretische Schwäche des Ansatzes darstellt. Im Deutschen könnten insbesondere der ausdrucksseitige Satzbauplan ,Subjekt - Prädikat - Dativobjekt - Akkusativobjekt‘ und die 17 dreiwertigen Präpositionalobjekts-Satzbaupläne ,Subjekt - Prädikat - Akkusativobjekt - Präpositionalobjekt‘ nicht vollständig analysiert werden (zu diesen siehe Kap. 5.2).

Um die Schwächen von Dowtys Ansatz zu beheben, postuliert Primus (2012, 2004: 379, 1999: 54-55) mehr Proto-Rollen, wie „Proto-Recipient“ (Primus 1999: 54). Bei Licht betrachtet stellt dieses Vorgehen jedoch nur einen Neuanfang der denotativen Semantik dar: Wie bereits in der Nachfolge Fillmores wird ein unzulängliches Set mehr und mehr erweitert. Solche Versuche verdeutlichen die eingangs geäußerte Kritik, nach der Dowtys Prototypenkonzept zwar in Bezug auf die Prototypik leistungsstark ist, das Kernproblem der deno- 
tativen Semantik - anstelle der Sprache die Welt zu analysieren - jedoch nur verdeckt und nicht löst.

Satz (8) ist aus den geschilderten Gründen ein Problem für Dowtys Konzept, signifikativ-semantisch jedoch unauffällig. Signifikativ-semantisch entwirft das Prädikat gebe einen HANDLungs-Sachverhalt. Das Subjekt trägt die Rolle HANDLUNGSTRÄGER, das Akkusativobjekt die bereits genannte Rolle HANDLUNGSGEGENSTAND und das Dativobjekt die Rolle HANDLUNGSBETROFFENER (GTA 2017: 7, 507).

Zur Absicherung bzw. Fundierung der Rollen kommen die Kriterien Produktivität und Alternation - in dieser Rangfolge - zur Anwendung, die sich an der Rolle des HANDLUNGSBETROFFENEN illustrieren lassen:

1. Produktivität: Eine semantische Rolle gilt als nachgewiesen, wenn sie produktiv belegt werden kann. D. h., wenn mindestens ein Beleg gefunden wird, in dem diese Rolle in der Umgebung eines Verbs realisiert wird, das die Rolle nicht in seiner Grundvalenz kodiert (GTA 2017: 269-279; Höllein 2019: 95-105). Für das Verb geben in (8) ist der HANDLUNGSBETROFFENE in der Grundvalenz vorgesehen, nicht aber für das Verb waschen:

(9) Die Beschaulichkeit der Schweiz hatte ihn wieder, niemand, der ihm während der Trainings das Auto wusch. (NZZ04/DEZ.01948 Neue Zürcher Zeitung, 11.12.2004, S. 62)

In Satz (9) wird das Verb waschen jedoch in ein dreiwertiges Satzbauplanzeichen mit dem Prototyp geben koerziert und produktiv mit einem Dativobjekt, das die Rolle HANDLUNGSBETROFFENER nachweist, realisiert. ${ }^{8}$ Wie restriktiv die Testvariante ist, illustriert folgender Beleg ([S153] des Korpustextes):

(10) sie sagt auf die Frage, was ihr in der Schule am besten gefalle

Das Verb sagen in Verbindung mit Präpositional ${ }_{\text {auf }+\mathrm{AKK}}$-objekt - weitere Beispiele sind antworten und reagieren - ist keine Instanz eines Satzbauplanzeichens (Höllein 2019: 179), sondern lediglich des nichtzeichenhaften Satzbauplans Subjekt/ TÄTIGKEITSTRÄGER - Prädikat/TÄTIGKEIT - Präpositional ${ }_{\text {auf+AKK-objekt/INAKTIV, da }}$ kein produktiver Beleg vorliegt, der die Bedeutung der Präpositionalobjektsrolle nachweisen könnte (die Rolle ist also inaktiv, siehe Kap. 3). Selbst der Korpusbe-

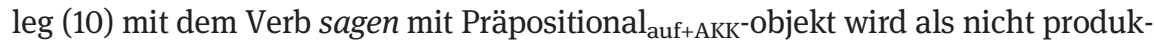

8 Der Begriff der Koerzion ist dabei eng an das Konzept von Goldberg (1995: 159) angelehnt (vgl. Höllein 2019: 40-50). 
tiv gewertet, da er in Müller (2012: 1568), einem korpusbasierten Präpositionswörterbuch, dessen Ziel es ist, die Grenzen des deutschen Sprachgebrauchs zu dokumentieren (Müller 2012: XIV), als Rektion gelistet ist. ${ }^{9}$

2. Alternation: Eine semantische Rolle gilt als nachgewiesen, wenn eine Alternation zweier Satzbauplanzeichen möglich ist. In der GTA (2017: 510) wird dies an folgenden Belegen exemplifiziert:

(11) Die Beamten backen der Redaktion einen Kuchen.

(12) Die Beamten backen für die Redaktion einen Kuchen.

Die Alternation von (11) vs. (12) verdeutlicht, dass der HANDLUNGSBETROFFENE in (11) die „signifikativ-semantisch offene Alternante“ (GTA 2017: 510) (d. h. die allgemeinere Bedeutung), die Rolle BENEFACTUM in (12) dagegen die „signifikativsemantisch spezifische, geschlossene Alternante“ (d.h. die spezifischere Bedeutung) verkörpert. Anders formuliert hat der durch das Dativobjekt realisierte

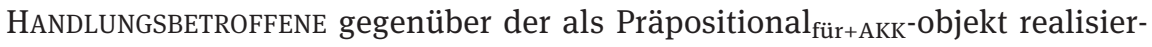
ten Rolle BENEFACTUM die allgemeinere Bedeutung.

Dass die Rollen durch die Kriterien Produktivität und Alternation fundiert werden, hat folgenden Grund: Produktivität ist ein Hinweis darauf, dass die semantische Rolle aktuell Quelle semantischer Kreativität ist, und Alternation darauf, dass zwischen produktiven Strukturen eine semantische Wahlmöglichkeit besteht. Somit ist Produktivität das übergeordnete Kriterium.

Da prototypische Adverbiale weder mit Objekten alternieren noch produktiv in dem Sinn sein können, dass sie einen Lesartwechsel erzwingen, sind Adverbiale nicht Träger signifikativ-semantischer Rollen. Adverbiale werden ohnehin semantisch klassifiziert, insofern sind sie zu Satzgliedern mit signifikativ-semantischen Rollen komplementär. ${ }^{10}$ Soweit Adverbiale in Satzbauplänen als Komplemente vorkommen, tragen sie nicht zur Produktivität des Satzbauplanzeichens bei.

9 Die Präpositionalobjektstheorie sowie die Methode zur Erhebung produktiver Belege sind in Höllein (2019) ausgeführt. Dort werden für Präpositionalobjekts-Präpositionen signifikativ-semantische Rollen angenommen, die durch produktive Belege wie im Text ausgeführt, nachgewiesen werden. Die Idee signifikativ-semantischer Rollen geht dabei auf Lerot (1982), Rostila (2007, 2005), Welke (1988) und die GTA 2017 zurück.

10 Eine signifikativ-semantische Neuklassifikation des bislang weitgehend denotativ-semantisch gefassten Adverbialsystems steht aus. Erste methodische und theoretische Vorschläge dazu sind z. B. von Coseriu (1970: 14) eingebracht worden. Für eine Zusammenfassung siehe Höllein (2019: 134-137). 


\section{Satzbaupläne als Zeichen (und Nichtzeichen)}

Die Annahme grammatischer (syntaktischer) einzelsprachlicher Grundstrukturen - terminologisch: Satz(bau)pläne, Satzmuster, Satzmodelle, Satztypen, Grundmodelle, Satzschemata, Valenzrealisierungsmuster, Kasusrahmen, Argumentstrukturmuster usw. - hat eine lange Forschungstradition, die in Deutschland nach Brinkmann (1971: 520) auf Berthold Delbrück und Karl Bühler, nach Engelen (1975/1: 23) gar auf Hermann Paul und Otto Behaghel zurückgeht.

Nach frühen Versuchen, grammatische Grundstrukturen auch inhaltlich zu motivieren (z. B. Admoni 1974 und 1982 [1. Aufl. 1960]; Brinkmann 1971: 519-606 und vor allem Engelen 1975/1-2), ging man im Sog der sich rasant entwickelnden Valenztheorie dazu über, Satzbaupläne auf Verbvalenzbasis zu entwerfen (Engel 1970; Schumacher 1975; KVL 1978; Götze 1979: 137-284; Erben 1980: 257-267; Tarvainen 1981: 102-112; Helbig 1982: 66-87) und sie als rein grammatische Grundstrukturen aufzufassen. Dabei wurde nicht nur das großartige und immer noch aktuelle Modell von Engelen (1975/1-2) ,verschüttet', sondern es blieb auch der beeindruckende, die Konstruktionsgrammatik vorwegnehmende Ansatz von Ickler (1990) weitgehend unberücksichtigt. Was Ickler (1990: 9) nachweisen konnte, war „daß der Kasusrahmen einen gewissen Teil der Satzsemantik eigenständig, d. h. unabhängig vom Verb signalisiert.“ Daraus, wie übrigens auch aus dem Ausdrucksmodell-Konzept von Feilke (1996: 211-256), folgt, dass die denotative Semantik, die - unverständlicherweise - auch von allen Schulen der Konstruktionsgrammatik praktiziert wird, per se zum Scheitern verurteilt ist. Denn in der denotativen Semantik ,werden die semantischen Rollen unabhängig von konkreten einzelsprachlichen Kriterien definiert: statt Kasusrahmen werden Sachverhalte klassifiziert.“ (Ickler 1990: 14). M. a. W., ein einzelsprachunabhängiges System semantischer Grundstrukturen ließe sich nur dann etablieren, wenn die einzelsprachlichen Systeme grammatischer Grundstrukturen keinen (signifikativ-semantischen) Beitrag zur Satzbedeutung leisten würden.

Doch auch die rein valenzgrammatische Satzbauplan-Forschung kommt in die Bredouille: „Wenn ein Satzbauplan nichts anderes ist als die Realisierung eines von der Valenz des Verbs bestimmten Programms, dann ist die Valenz primär, und der Begriff des Satzbauplans kann [. . . ] in der Theorie [. . .] als redundant angesehen und somit ohne Weiteres entbehrt werden." (Vuillaume 2003: 496)

Eine (im doppelten Sinne) sinnvolle Modellierung von Satzbauplänen bedeutet also die Begründung eines Systems einzelsprachlicher grammatischer Grundstrukturen, die alle einen (signifikativ-)semantischen Differenzwert ( $v a$ leur) haben. Lässt sich einer grammatischen Grundstruktur ein (signifikativ-)semantischer Differenzwert zuordnen, der diese grammatische Grundstruktur von 
allen anderen grammatischen Grundstrukturen desselben Systems semantisch unterscheidet, könnte möglicherweise bereits von Satzbauplanzeichen, d.h. von „complex and mostly schematic“ Konstruktionen (Croft 2001: 17) bzw. von „komplexe(n) Zeichenschemata im Sinne Saussures“ (Schneider 2014: 364), gesprochen werden.

Doch, wie in Kap. 2 ausgeführt, bevorzugen wir einen strengeren, da empirisch zweifelsfrei nachweisbaren Zeichenbegriff für Grundstrukturen als komplexe grammatisch-semantische Zeichenschemata: Nur wenn sich semantische Rollen durch Produktivität und Alternation nachweisen lassen, d. h. nur wenn sie aktiv sind, sprechen wir von zeichenhaften Satzbauplänen, also von ,echten“ Satzbauplanzeichen. In Ausnahmefällen, z. B. bei Ergativität (Typ: Mich friert/ hungert, siehe unten), sind nämlich semantische Rollen inaktiv (= nicht mehr oder noch nicht aktiv) oder ihre Produktivität lässt sich empirisch nicht nachweisen. In diesen Fällen sprechen wir von nichtzeichenhaften Satzbauplänen. ${ }^{11}$ Im Folgenden soll diese Auffassung kurz vorgestellt werden (mehr dazu in GTA 2017 und Höllein 2019).

Die grammatisch-semantischen Grundstrukturen des Gegenwartsdeutschen werden auf der Grundlage der GTA 2017 modelliert (siehe auch Kap. 4.1): Nach dieser Syntaxtheorie besteht die grammatische Grundstruktur eines Satzes aus dem Hauptprädikat (= Prädikat eines einfachen Satzes oder Hauptsatzprädikat eines Satzgefüges) und dessen Komplementen. Semantisch (= signifikativ-semantisch) stellt ein Satz einen qua Hauptprädikat entworfenen und qua Hauptprädikat und Komplementen realisierten einzelsprachlich perspektivierten Sachverhalt (Welke 2005: 92-98), ein Szenario (Fischer 2003: 28-30), dar.

Betrachten wir nun den folgenden Satz (kursiviert) und eine mögliche alternative Formulierung mit der Beschreibung ihrer Grundstrukturen: ${ }^{12}$

(13) Ich schicke voraus, daß ich kein Kunstkenner bin, sondern Laie. Ich habe oft bemerkt, daß mich der Inhalt eines Kunstwerkes stärker anzieht als dessen formale und technische Eigenschaften, auf welche doch der Künst-

11 Entsprechend werden in Kap. 5.2 die Satzbauplanzeichen getrennt von den nichtzeichenhaften Satzbauplänen gelistet.

12 Die semantische Grundstruktur (= semantisches Prädikat + signifikativ-semantische Rollen) wird durch Kapitälchen gekennzeichnet. Signifikativ-semantische Grundstrukturen - inkl. der semantischen Subjektrollen HANDLUNGSTRÄGER, VORGANGSTRÄGER und ZUSTANDSTRÄGER - wurden bereits in Fleischmann (1985) entwickelt und angewandt, ohne dass dies in der Linguistik rezipiert worden wäre. Welke, der die signifikative Semantik begründet und bereits in Welke (1994) die Rollen VORGANGSTRÄGER und ZUSTANDSTRÄGER eingesetzt hat, scheint Fleischmann auch nicht zu kennen. Die nachfolgenden Analysen inkl. der semantischen Rollen sind alle der GTA (2017) entnommen. 
ler in erster Linie Wert legt. Für viele Mittel und manche Wirkungen der Kunst fehlt mir eigentlich das richtige Verständnis. Ich muß dies sagen, um mir eine nachsichtige Beurteilung meines Versuches zu sichern. (Freud Moses, S. 172)

$\begin{array}{lll}\text { Dativobjekt } & \text { - Prädikat }- & \text { Subjekt } \\ \text { ZUSTANDSBETROFFENER } & - \text { ZUSTAND }- & \text { ZUSTANDSTRÄGER } \\ \text { mir } & \text { fehlt } & \text { das richtige }\end{array}$

(13') Für viele Mittel und manche Wirkungen der Kunst bringe ich eigentlich kein richtiges Verständnis auf.

$\begin{array}{lll}\text { Subjekt } & \text { - Prädikat }- & \text { Akkusativobjekt } \\ \text { HANDLUNGSTRÄGER } & \text { - HANDLUNG }- & \text { HANDLUNGSGEGENSTAND } \\ \text { ich } & \text { bringe. . . auf } & \text { kein richtiges Verständnis für. . . }\end{array}$

Im Originalbeleg nimmt Freud keine Handlungsperspektive ein. Mit Hilfe des FEHLEN-Szenarios und der grammatischen Grundstruktur ,Dativobjekt-Prädikat-Subjekt' positioniert er sich als ZUSTANDSBETROFFENER, als jemand, der sich beobachtend feststellen muss, dass er über eine bestimmte innere Disposition nicht verfügt, dass er eben kein ZUSTANDSTRÄGER ist.

Diese Szenierung (= einzelsprachliche Sachverhaltsperspektivierung) passt gut zur Captatio Benevolentiae, die hier von Freud angestrebt wird. Die alternative Handlungsperspektivierung mit Hilfe des AUFBRINGEN-Szenarios hätte weniger gut zur Textsorte gepasst.

Szenierungsalternativen lassen sich nicht nur lexikalisch - durch Hauptprädikatswechsel - , sondern auch grammatisch herstellen:

(14) Jetzt hat sich die Witwe sofort wieder beruhigt. (Haas Silentium, S. 80)

$\begin{array}{ll}\text { Subjekt } & \text { - Prädikat - } \\ \text { ENDO-VORGANGSTRÄGER } & \text { - ENDO-VORGANG - } \\ \text { Die Witwe } & \text { beruhigt sich }\end{array}$

(14') Der Brenner beruhigt die Witwe.

$\begin{array}{lll}\text { Subjekt } & \text { - Prädikat } & \text { - Akkusativobjekt } \\ \text { HANDLUNGSTRÄGER } & \text { - HANDLUNG } & \text { HANDLUNGSGEGENSTAND } \\ \text { Der Brenner } & \text { beruhigt } & \text { die Witwe }\end{array}$


(14") Die Witwe wird (vom Brenner) beruhigt.

$\begin{array}{lll}\text { Subjekt } \quad- & \text { Prädikat }- & \\ \text { EXO-VORGANGSTRÄGER }- & \text { EXO-VORGANG }- & \text { (EXO-VORGANGSAUSLÖSER) } \\ \text { Die Witwe } & \text { wird beruhigt } & \text { (vom Brenner })\end{array}$

Die drei Szenarios - das SiCH BERUHIGEN-, das BERUHIGEN- und das BERUHIGT-WERDEN-Szenario - stellen drei verschiedene Perspektivierungen derselben Situation dar. Je nachdem, welche semantische Rolle das Subjekt indiziert, wird die Situation aus der Perspektive des HANDLUNGSTRÄGERS oder aus der von zwei verschiedenen VORGANGSTRÄGERN szeniert. ${ }^{13}$

Wie die Beispiele andeuten, lässt sich signifikativ-semantisch das Potenzial grammatischer Grundstrukturen gewissermaßen kontrastiv beschreiben. Es geht nicht, wie bei der denotativen Semantik, um die grammatische Umordnung derselben außer(einzel)sprachlichen semantischen Rollen, mit denen die Autonomie der Semantik quasi vorgetäuscht wird, sondern um das System einzelsprachlich realisierbarer semantischer Differenzwerte: Die semantische Leistung jedes einzelnen Satzbauplans einer Einzelsprache unterscheidet sich von der jedes anderen.

Wie erwähnt, reicht uns der nachweisbare semantische Differenzwert aber nicht aus, um von einem Satzbauplanzeichen zu sprechen. Denn die semantische Differenz zwischen einem Satzbauplan A und einem Satzbauplan B bedeutet noch nicht, dass A oder B zeichenhaft wären. Von einem Satzbauplanzeichen lässt sich erst sprechen, wenn ein Satzbauplan die Zielstruktur von Innovationen oder gar von Sprachwandel darstellt. Anders gesagt: Gäbe es keine Satzbauplanzeichen, könnte es weder satzbauplanbezogene Innovationen noch satzbauplanbezogenen Sprachwandel geben.

13 Um die Unterschiede zwischen passivischer und medialer Realisierung zu erfassen, wurden in der GTA 2017 in Anlehnung an Haspelmath (1993: 108) zwei Typen von sprachlichen Perspektivierungen von Zustandsveränderungen eingeführt: exo- und endoaktiv: In ,exoaktiver Perspektive (kurz: EXO) wird eine Zustandsveränderung als äußere Einwirkung, als die Einwirkung einer externen Kraft (eines ,Auslösers') dargestellt. Dies ist per definitionem der Fall in Handlungssätzen (mit AGENS-Subjekt), aber auch in passivischen Vorgangssätzen. Umgekehrt heißt ,endoaktiv‘ (kurz: ENDO), dass eine Zustandsveränderung einzelsprachlich als ein spontanes Geschehen, als ein Geschehen ohne äußere Einwirkung, wiedergegeben wird. Bei ENDO-Vorgängen kann es also keinen VORGANGSAUSLÖSER geben. Um die Textanalyse und die Beschreibung der Satzbauplanzeichen nicht unnötig zu verkomplizieren, wird im vorliegenden Beitrag auf die Unterklassifizierung von VoRGANG verzichtet. 
Betrachten wir hierzu den folgenden Vers von Georg Trakl mit transitivem schweigen (Beleg nach Sauermann 1985: 337):

(15) Frühlingsgewölke steigen über die finstere Stadt, Die der Mönche edlere Zeiten schweigt

Zum konventionalisierten Wortschatz im heutigen Deutsch gehört das intransitive Verb schweigen und $\mathrm{zu}$ den konventionalisierten gegenwartsdeutschen Satzbauplänen gehört der im Trakl-Vers realisierte transitive Satzbauplan ,Subjekt/HANDLUNGSTRÄGER - Akkusativobjekt/HANDLUNGSGEGENSTAND“. „Die Innovation, der Normverstoß besteht lediglich in der normwidrigen Zuordnung eines normalen VT [= Valenzträgers] zu einer normalen Grundstruktur. Dadurch, dass der Makrorealisierung des Zweitaktanten (!) gar kein Zweitaktant in der Valenzpotenz von schweigen entspricht, wird bei der Leserin / beim Leser der Eindruck (strukturell) erzwungen, dass der VT zweiwertig ist. Der Zweitaktant wird nicht via Valenz, sondern via Valenzrealisierungsstruktur ,zugewiesen', die Innovation geht aus dem Konflikt zwischen ,Strukturerwartung“ und ,Strukturrealität‘ (Welke 1995: 171) hervor.“ (Ágel 2000: 270)

Innovationen beschränken sich keinesfalls auf die Dichtersprache, sondern kommen auch im sprachlichen Alltag vor:

(16) Im Gegensatz zum Medellin-Kartell war das Cali-Kartell vertikal organisiert und definierte sich auf gemeinsame Kooperationen. (http://de.wikipedia. org/wiki/Cali-Kartell, 09.12.2014)

Das Hauptprädikat sich definieren bedient sich hier des Satzbauplans, dem konventionell Verben wie hoffen oder sich freuen $a u f_{+\mathrm{AKK}}$ angehören. Entsprechend

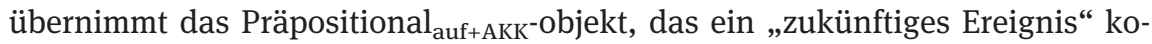
diert, die semantische Rolle PRospectum (Höllein 2019: 180).

Scheinbar derselbe Fall liegt im folgenden Beleg vor:

(17) $\operatorname{Er}[=$ Krüger, VÁ/DH] fuhr $z u$ den Ziegelwerkern hinaus. »Ihr macht hier viel, aber schlechte Ziegel, meine Freunde.« »Hoho, was für Weisheiten!« die Ziegler erboten sich, gute, dafür aber weniger Ziegel zu machen. »Alle Tage, Sportsfreund!« Krüger bestand auf viel Ziegel und gute Ziegel, aber in dieses Paradies schien kein Weg zu führen. (Strittmatter Bienkopp, S. 264)

Doch hier geht es nicht um eine okkasionelle Innovation, sondern um Sprach-

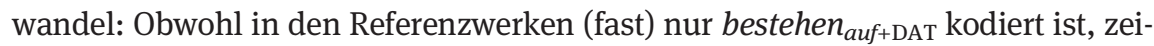
gen Tests mit jungen Probandinnen und Probanden, dass bestehen auf $+\mathrm{DAT}_{\text {nicht }}$ 
nur veraltet ist, sondern von der jungen Generation mehrheitlich sogar abgelehnt wird. ${ }^{14}$ Der Wechsel des Satzbauplans bedeutet dabei nicht nur einen grammatischen, sondern auch einen semantischen Wandel: Das Präpositional ${ }_{\text {auf }+ \text { DAT }}$-objekt der älteren Generation trägt die semantische Rolle BASIS (Höllein 2019: 182-185, 189-192), deren Bedeutung als „Grundlage des Szenarios“ paraphrasiert wird, das Präpositional ${ }_{\mathrm{auf}+\mathrm{AKK}}$-objekt der jüngeren Generation die semantische Rolle PRosPectum. Der Wandel der grammatischen Grundstruktur mit Präpositional ${ }_{\text {auf }+ \text { DAT-Objekt }}>$ Präpositional $_{\text {auf }+A K K}$-objekt indiziert also lediglich den semantischen Wandel BASIS $>$ PROSPECTUM. ${ }^{15}$

Sowohl der Beleg mit sich definieren ${ }_{\text {auf }+ \text { AKK }}$ als auch der Sprachwandel mit bestehen $_{\text {auf }+\mathrm{AKK}}$ zeigen, dass es Satzbauplanzeichen gibt: Grundstrukturen, die aktiv (produktiv) sind und deshalb mögliche Zielstrukturen von Innovation und Wandel darstellen.

Kein Satzbauplanzeichen liegt dagegen bei der sog. umpolenden Verwendung des Empfindungsverbs frieren vor: ${ }^{16}$

(18) Mich friert.

Akkusativobjekt - Prädikat -

ZUSTANDSGEGENSTAND - ZUSTAND -

Mich friert

$14 \mathrm{Zu}$ Details des 2012 in Kassel mit 23 muttersprachlichen Studierenden durchgeführten Tests siehe GTA (2017: 537-538). Der Kasseler Test wurde 2018 in Leipzig mit 18 muttersprachlichen Studierenden wiederholt. Die Ergebnisse waren im Wesentlichen identisch (siehe auch Ágel 2019). Daraus kann man vorsichtig schließen, dass zumindest in der Standardsprache ein Sprachwandel vorliegt. Wir können aber nicht ausschließen, dass in bestimmten Dialekten das Präpositional ${ }_{\text {auf }+\mathrm{AKK}}$-objekt älter ist. Wir danken einem/r anonymen GutachterIn für den Hinweis auf das Schwäbische.

15 Diese Wandelphänomene von semantischen Präpositionalobjektsrollen (Nischen) werden in Höllein (2019: 82-89) detaillierter über das Konzept des Nischentransits erfasst. Mit diesem Konzept sind Fälle scheinbarer Rollensynonymie - wie z. B. bei der Rolle BASIs, die sowohl mit Präpositional ${ }_{\text {auf }+\mathrm{AKK}}$-objekt als auch mit Präpositional ${ }_{\text {auf }+\mathrm{DAT}}$-objekt steht, erklärbar: Es handelt sich nicht um systematische Synonymie, sondern um eine historisch-temporäre Überschneidung der Bedeutung.

16 Sprachenübergreifend unterscheidet Bossong (1992: 109-110) zwei Grundtechniken der grammatischen Kodierung von Empfindungsprädikaten: Umpolung, bei der man „ihrer spezifischen Semantik dadurch Rechnung tragen (kann), daß man sie nicht mehr als Subjekte, sondern als Objekte kodiert“ und Generalisierung, bei der man „von ihrer spezifischen Semantik abstrahieren (kann) und sie dem herrschenden Prototyp der Handlungsverben angleichen [. . .].“ (Bossong 1992: 109). 
Zwar steht die umpolende Verwendung in Opposition zu der sog. generalisierenden Verwendung von frieren:

(19) Ich friere.

$\begin{array}{ll}\text { Subjekt } & \text { - Prädikat - } \\ \text { TÄTIGKEITSTRÄGER } & \text { - TÄTIGKEIT - } \\ \text { Ich } & \text { friere }\end{array}$

Doch ist die umpolende Verwendung - im Gegensatz zur generalisierenden zweifelsohne nicht mehr aktiv. ${ }^{17}$ Der Satzbauplan von mich friert ist demnach nicht zeichenhaft, während ich friere die Realisierung eines Satzbauplanzeichens darstellt. ${ }^{18}$

Der aktuelle Status eines Satzbauplans ist einerseits natürlich immer eine historische Momentaufnahme, andererseits aber auch eine, die den Forschungsstand widerspiegelt. Denn einerseits besteht der satzbauplanbezogene Sprachwandel darin, dass nichtzeichenhafte Satzbaupläne zeichenhaft werden oder umgekehrt. ${ }^{19}$ Andererseits kann durch neue empirische Forschungen die Zeichenhaftigkeit eines nichtzeichenhaft geglaubten Satzbauplans nachgewiesen werden.

\section{Theorievergleichende Textanalyse}

\subsection{Aufbereitung des Textes: von Textgliedern zu Grundstrukturen}

Um syntaktisch-semantische Grundstrukturen analysieren zu können, muss man sie zuerst in Texten identifizieren. Es sei denn, man ist der Auffassung, dass eigene Beispielsätze reichen. Da die 50-jährige Geschichte der erfolglosen

17 Im Sinne unserer obigen Definition liegt hier keine Alternation vor, weil die umpolende Verwendung des Empfindungsverbs frieren nicht mehr produktiv ist.

18 Im Unterschied zu (transitiven) Verben, die im Aktiv ein Tun, durch das etwas bewirkt/verursacht wird, d.h. eine HANDLUNG, kodieren, beschreiben (intransitive) Verben im Aktiv ein ,bloßes‘ Tun, ohne etwas zu bewirken, d. h. eine TäTigKeIT (Welke 2005: 179-188). Im Passiv drücken beide VORGANG aus. In Kap. 4.2 werden auch die signifikativ-semantischen Prädikatsrollen definiert und exemplifiziert.

19 Deshalb endet die Grammatikalisierung von Adverbialbestimmungen zu Präpositionalobjekten nicht mit der Herausbildung von Präpositionalobjekten. Denn je nach Status des Präpositionalobjekts (+/-aktiv) kann durch Weitergrammatikalisierung dessen Satzbauplan zum Zeichen werden oder eben seinen Zeichenstatus einbüßen (GTA 2017: 528-532). 
denotativen Rollenanalysen jedoch sehr deutlich gegen die eigenen Beispielkonstruktionen spricht und da unser Ansatz sowieso textbasiert ist, müssen wir an dieser Stelle den Weg vom Textganzen zu den syntaktisch-semantischen Grundstrukturen gehen.

Die theoretische Grundlage unserer Analyse bildet die GTA 2017, eine deszendente Syntax des Gegenwartsdeutschen. Syntaxen und generell Grammatiken sind traditionell aszendent, ,von unten nach oben' gerichtet: Einer Wortgrammatik folgt eine Satzgrammatik und dieser evtl. eine Textgrammatik. In der GTA 2017 wird das grammatische System ,von oben nach unten“ - von der Text- (Textglieder) über die Satz- (Satzglieder) zur Wortgruppenebene (Wortgruppenglieder) modelliert. ${ }^{20}$

Um die syntaktisch-semantischen Grundstrukturen eines Textes identifizieren zu können, sind folgende methodische Schritte notwendig:

1) Segmentierung des Textes in Textglieder, darunter in Sätze;

2) Identifizierung aller Haupt- und Nebenprädikate und deren syntaktischer Umgebung (Komplemente und Supplemente);

3) Bestimmung der Satzgliedwerte;

4) Bestimmung der signifikativ-semantischen Rollen.

Wir gehen hier nur auf 1) und 2) ein. Die Bestimmungen der Satzgliedwerte und der signifikativ-semantischen Rollen sind Kap. 4.2 und $4.3 \mathrm{zu}$ entnehmen.

Ad 1): In der GTA werden drei Typen von Textgliedern angenommen und ausführlich begründet (GTA 2017: 24-28 und 61-246): Satz, Nichtsatz und Kohäsionsglied. ${ }^{21}$

Das Textglied ,Satz‘ enthält ein einziges Hauptprädikat (= Prädikat eines einfachen Satzes oder Hauptsatzprädikat eines Satzgefüges) und hat qua Klammerbildungspotenzial des Hauptprädikats eine Felderstruktur. ${ }^{22}$ Das Textglied ,Nichtsatz hat kein Hauptprädikat und keine Klammerstruktur. Das Textglied ,Kohäsionsglied' stellt einen syntaktisch-semantischen Relator zwischen zwei Sätzen, zwischen zwei Nichtsätzen, zwischen einem Satz und einem Nichtsatz oder eben zwischen ganzen Textsequenzen dar, die sich wiederum als Satz- und Nichtsatzverbindungen bzw. Textverkettungen beschreiben lassen. Topologisch stehen Ko-

20 Die GTA ist also keine Textgrammatik, sondern eine ,auf den Kopf gestellte“ Syntax, die auf der Textebene ansetzt. Die wichtigsten Theoriebausteine der GTA wurden in Ágel (2019) kurz zusammengefasst.

21 Unter ,Satz' (ohne Attribuierung) wird immer der grammatische Satz verstanden. Von diesem zu unterscheiden ist der orthographische Satz (Ganzsatz).

22 Nebensätze habe keine Klammer- und Felderstruktur (GTA 2017: 104-114). Klammerbildung ist das Markenzeichen von Hauptprädikaten. 
häsionsglieder in der sog. Zwischenstelle zwischen Sätzen und/oder Nichtsätzen (GTA 2017: 79-82), oder sie sind in die topologische Struktur des jeweiligen Satzes integriert.

Betrachten wir folgende Belege aus dem analysierten Text: ${ }^{23}$

(20) Dass Ylva nun direkt um die Ecke eingeschult wurde, ist alles andere als selbstverständlich, denn sie wohnt in Neukölln.

(21) Spätestens seitdem im Jahr 2006 die Lehrer der Rütli-Schule öffentlich um Hilfe riefen und ihre Schule so deutschlandweit in die Schlagzeilen brachten, steht der Stadtteil Neukölln für ein Umfeld, in dem Eltern sich ihr Kind lieber nicht vorstellen möchten. Das Schlagwort, das ihre Ängste konzentriert, lautet: Brennpunktschule. Nun stehen viele dieser Brennpunktschulen ausgerechnet dort, wo junge Eltern mittlerweile gerne wohnen.

(22) Vorsicht, Brennpunkt!

Beleg (20) enthält zwei Sätze - ein Satzgefüge und einen einfachen Satz und das Kohäsionsglied denn in der Zwischenstelle. Dagegen ist das Kohäsionsglied nun in Beleg (21), das den zweiten mit dem dritten Satz(gefüge) verbindet, in das Vorfeld des dritten Satzes integriert. Zwar steht es also im dritten Satz, es ist jedoch kein Satz-, sondern ein Textglied. ${ }^{24}$ Schließlich enthält Beleg (22) zwei Nichtsätze. Kohäsionsglieder und Nichtsätze spielen für die weitere Analyse keine Rolle. Übrig bleiben die Sätze: einfache Sätze und Satzgefüge.

Ad 2): Bei einfachen Sätzen, die per definitionem nur ein Hauptprädikat enthalten, bedarf die Identifizierung von dessen syntaktischer Umgebung keiner besonderen Rekonstruktion: ${ }^{25}$

[S10] sie wohnt in Neukölln

23 In der GTA werden folgende Markierungskonventionen verwendet, um die drei Sorten von Textgliedern optisch leicht identifizieren zu können: Bei Sätzen werden die Hauptprädikate fett markiert. Nichtsätze sind Punkt-Strich unterstrichen, Kohäsionsglieder unterstrichen. 24 Das Wort nun kommt im Text nicht nur als Kohäsionsglied, sondern auch als Temporaladverbial vor: Beispielsweise in Beleg (20) oder auch im folgenden Beleg: Viele Eltern aus BerlinNeukölln schulen ihre Kinder lieber in anderen Vierteln ein. Eine Initiative versucht nun, dagegen anzukämpfen.

25 Die folgende Nummerierung entspricht der Satznummerierung des analysierten Textes (siehe Kap. 4.3). [S10] z. B. ist der 10. Satz im Text, [S9a] das rekonstruierte Nebenprädikat von [S9] mit seiner syntaktischen Umgebung usw. 
Bei Satzgefügen wird zuerst das Hauptprädikat mit seiner syntaktischen Umgebung, d. h. der Satz selbst, angeführt: ${ }^{26}$

[S9] Dass Ylva nun direkt um die Ecke eingeschult wurde, ist alles andere als selbstverständlich,

[S15] Spätestens seitdem im Jahr 2006 die Lehrer der Rütli-Schule öffentlich um Hilfe riefen und ihre Schule so deutschlandweit in die Schlagzeilen brachten, steht der Stadtteil Neukölln für ein Umfeld, in dem Eltern sich ihr Kind lieber nicht vorstellen möchten.

[S16] Das Schlagwort, das ihre Ängste konzentriert, lautet: Brennpunktschule.

[S17] viele dieser Brennpunktschulen stehen ausgerechnet dort, wo junge Eltern mittlerweile gerne wohnen.

Anschließend werden die Nebenprädikate mit ihrer syntaktischen Umgebung rekonstruiert:

[S9a] Ylva wurde nun direkt um die Ecke eingeschult

[S15a] Eltern möchten sich ihr Kind in dem [Umfeld] lieber nicht vorstellen

[S15b] die Lehrer der Rütli-Schule riefen im Jahr 2006 öffentlich um Hilfe

[S15c] die Lehrer der Rütli-Schule brachten ihre Schule so deutschlandweit in die Schlagzeilen

[S16a] Das [Schlagwort] konzentriert ihre Ängste

[S17a] junge Eltern wohnen wo [dort] mittlerweile gerne

Der analysierte Zeitungstext enthält insgesamt 156 Sätze. Zu deren Hauptprädikaten mit ihrer syntaktischen Umgebung kommen 115 Rekonstruktionen von Nebenprädikaten mit ihrer syntaktischen Umgebung hinzu. Unsere Ergebnisse hinsichtlich des Vergleichs des Potenzials von denotativ- vs. signifikativ-semantischen Rollen basieren also auf insgesamt 271 analysierten Satzbauplanrealisierungen.

26 Bei [S17] enthält die Rekonstruktion das integrierte Kohäsionsglied nun ja nicht mehr, weil nun trotz seiner topologischen Integration keinen grammatischen Wert auf Satz-, sondern nur auf Textebene hat. 


\subsection{Liste der denotativ- vs. signifikativ-semantischen Rollen}

Eine theorievergleichende Textanalyse, wie sie in Kap. 4.3 vorgelegt wird, setzt je eine Liste denotativ-semantischer und signifikativ-semantischer Rollen voraus. Für die denotativ-semantische Analyse des Zeitungstextes haben wir mit dem Rollenset von Fillmore (2003: 464) gearbeitet. ${ }^{27}$ Dabei wurden seine englischen Beispiele ins Deutsche übersetzt (siehe Tab. 1):

Tab. 1: Denotativ-semantische Rollen (nach Fillmore 2003: 464).

\begin{tabular}{lll}
\hline Rollenbezeichnung & Paraphrase & Beispiele \\
\hline AGENT & ,the instigator of an event & John schrie. \\
\hline INSTRUMENT & ,the entity directly instrumental in causing a & $\begin{array}{l}\text { Der Stein hat den } \\
\text { Kotflügel eingedrückt. }\end{array}$ \\
\hline STIMULUS & ,the impetus or stimulus of a mental event & $\begin{array}{l}\text { Der Lärm machte den } \\
\text { Kindern Angst. }\end{array}$ \\
\hline PATIENT & $\begin{array}{l}\text {,the entity which, according to the verb’s } \\
\text { meaning, is treated as being in a state or as } \\
\text { undergoing a change of state }\end{array}$ & Die Statue schmolz. \\
\hline THEME & $\begin{array}{l}\text {,an entity to which the verb's meaning assigns a a } \\
\text { location or a change of location }\end{array}$ & Die Statue blieb dort. \\
\hline EXPERIENCER & $\begin{array}{l}\text {,the psychological locus of a sensory, cognitive } \\
\text { or emotional experience }\end{array}$ & Der Junge liebt Käse. \\
\hline
\end{tabular}

27 Eine Alternative wäre die semantische Rollenliste von von Polenz (2008: 170-172) gewesen, die von Lasch und Ziem in verschiedenen Publikationen (Ziem \& Lasch 2013: 124-125; Lasch 2016: 39-40) genutzt und um weitere denotativ-semantische Rollen ergänzt worden ist. Wir haben uns für Fillmore entschieden, da seine Publikation erstens neuer ist, zweitens sein Ansatz nicht nur der Prototyp, sondern nach wie vor Orientierungspunkt für die Forschung ist. Uns ist klar, dass zum einen Rollensets unter anderem von Fillmore (2006: 616) selbst, aber auch von anderen (Levin \& Rappaport Hovav 2008: 38-41), generell kritisiert werden und zum anderen immer wieder Vorschläge für neue denotative Rollen abseits der Sets gemacht worden sind. U. E. stellen solche Erweiterungen aber nur punktuelle Modifikationen dar, die an den systematischen Problemen der denotativen Semantik nichts ändern. Außerdem betrachten wir es nicht als unsere Aufgabe, ein denotativ-semantisches Rollenset unter Nutzung der denotativ-semantischen Fachliteratur zu entwerfen. 
Tab. 1 (fortgesetzt)

\begin{tabular}{lll}
\hline Rollenbezeichnung & Paraphrase & Beispiele \\
\hline CONTENT & ,the content of a cognitive state or event & $\begin{array}{l}\text { Wir erinnern ihre } \\
\text { Telefonnummer. }\end{array}$ \\
\hline BENEFICIARY & ,the entity on whose behalf an action is & $\begin{array}{l}\text { Wir haben alles für } \\
\text { dich getan. }\end{array}$ \\
\hline pourformed by the AGENT & ,the starting point of a motion“ & Er verließ das Haus. \\
\hline GOAL & ,the destination of a motion“ & Er kam nach Hause. \\
\hline PATH & ,the trajectory of a motion‘ & $\begin{array}{l}\text { Wir gingen den Bach } \\
\text { entlang. }\end{array}$ \\
\hline
\end{tabular}

Für die signifikativ-semantische Analyse des Zeitungstextes konnten wir im nichtpräpositionalen Bereich zwar auf Vorarbeiten zurückgreifen (Fleischmann 1985; GTA 2017, Ágel 2019; Höllein 2017; Welke 2005, 2011), die Rollendefinitionen stammen jedoch von uns. Die Modellierung des präpositionalen Bereichs erfolgt nach Höllein (2019: 288-290). ${ }^{28}$ Da das komplette Set der signifikativ-semantischen Rollen neu ist, werden sie nicht nur benannt, sondern auch definiert und ausführlich exemplifiziert. Dabei werden, um die beiden Rollensets wenigstens ansatzweise vergleichbar zu machen, alle obigen Beispiele aus Fillmore 2003 bzw. die Beispiele aus Levin/Rappaport Hovav (2008: 28) und aus Levin (1999: 224), die in Kap. 2 zitiert wurden, signifikativ-semantisch eingeordnet. Die übrigen Beispiele stammen mehrheitlich aus dem analysierten Text.

Der Übersichtlichkeit halber präsentieren wir das Set der signifikativsemantischen Rollen in zwei Tabellen: Tabelle $2 \mathrm{~b}$ enthält die Präpositional (objekts)rollen, Tabelle 2a alle anderen Rollen, die wir, als Verlegenheitslösung, Kernrollen genannt haben:

28 Köpcke hat mit Hinze bereits 2011 klar formuliert, dass die herrschende Auffassung von der semantischen Leere der PO nicht zutreffend ist: „Das [. . .] Problem, das [. . . ] hinsichtlich der Abgrenzung gegenüber den POs entsteht, betrifft die unzulässige Schlussfolgerung, Adverbiale hätten eine spezifische Semantik und POs eben nicht. Diese Verallgemeinerung trifft, wenn überhaupt, nur für die jeweiligen Prototypen zu.“ (Köpcke/Hinze 2011: 62). 
Tab. 2a: Signifikativ-semantische Kernrollen.

\begin{tabular}{|c|c|c|}
\hline Rollenbezeichnung & Paraphrase & Beispiele \\
\hline HANDLUNG & $\begin{array}{l}\text {,die eigenaktive (+CONTR) } \\
\text { und dynamische (+DYN) } \\
\text { semantische Rolle eines } \\
\text { transitiven Prädikats‘ }\end{array}$ & $\begin{array}{l}\text { Der Stein hat den Kotflügel eingedrückt. } \\
\text { Der Lärm stört die Kinder. } \\
\text { Der Junge liebt Käse. } \\
\text { Wir erinnern ihre Telefonnummer. } \\
\text { Er verließ das Haus. } \\
\text { Der Ingenieur zerstört/bemalt/bewegt/ } \\
\text { baut/wäscht/überquert/sieht die } \\
\text { Brücke. } \\
\text { Die Hitze schmolz die Statue. }\end{array}$ \\
\hline HANDLUNGSTRÄGER & $\begin{array}{l}\text {,gegenstandsbezogene } \\
\text { semantische Rolle des } \\
\text { Subjekts eines } \\
\text { Handlungsprädikats، }\end{array}$ & $\begin{array}{l}\text { Der Stein hat den Kotflügel eingedrückt. } \\
\text { Der Lärm stört die Kinder. } \\
\text { Der Junge liebt Käse. } \\
\text { Wir erinnern ihre Telefonnummer. } \\
\text { Er verließ das Haus. } \\
\text { Der Ingenieur zerstört/bemalt/bewegt/ } \\
\text { baut/wäscht/überquert/sieht die } \\
\text { Brücke. } \\
\text { Die Hitze schmolz die Statue. }\end{array}$ \\
\hline HANDLUNGSGEGENSTAND & $\begin{array}{l}\text {,gegenstandsbezogene } \\
\text { semantische Rolle des } \\
\text { Akkusativobjekts eines } \\
\text { Handlungsprädikats، }\end{array}$ & $\begin{array}{l}\text { Der Stein hat den Kotflügel eingedrückt. } \\
\text { Der Lärm stört die Kinder. } \\
\text { Der Junge liebt Käse. } \\
\text { Wir erinnern ihre Telefonnummer. } \\
\text { Er verließ das Haus. } \\
\text { Der Ingenieur zerstört/bemalt/bewegt/ } \\
\text { baut/wäscht/überquert/sieht die } \\
\text { Brücke. } \\
\text { Die Hitze schmolz die Statue. }\end{array}$ \\
\hline HANDLUNGSBETROFFENER & $\begin{array}{l}\text {,gegenstandsbezogene } \\
\text { semantische Rolle des } \\
\text { Dativobjekts eines } \\
\text { Handlungsprädikats‘ }\end{array}$ & $\begin{array}{l}\text { Der Ingenieur baut seiner Frau eine } \\
\text { Brücke. }\end{array}$ \\
\hline HANDLUNGSVERMITTLER & $\begin{array}{l}\text {,gegenstandsbezogene } \\
\text { semantische Rolle des } \\
\text { Akkusativobjekts eines Acl- } \\
\text { Handlungsprädikats“ }\end{array}$ & Ich sehe ihn die Einfahrt ausbessern. \\
\hline
\end{tabular}


Tab. 2a (fortgesetzt)

\begin{tabular}{|c|c|c|}
\hline Rollenbezeichnung & Paraphrase & Beispiele \\
\hline HANDLUNGSAUSLÖSER & $\begin{array}{l}\text {,gegenstandsbezogene } \\
\text { semantische Rolle des } \\
\text { Präpositional } \\
\text { einon+DAT-objekts } \\
\text { eindlungsprädikats‘ }\end{array}$ & Ich lasse von ihm das Auto holen. \\
\hline HANDLUNGSSACHVERHALT & $\begin{array}{l}\text {,sachverhaltsbezogene } \\
\text { semantische Rolle des } \\
\text { Subjekts oder des } \\
\text { Akkusativobjekts eines } \\
\text { Handlungsprädikats“ }\end{array}$ & $\begin{array}{l}\text { Ständig dagegen anzukämpfen, macht } \\
\text { ihn müde. } \\
\text { Eine Initiative versucht nun, dagegen } \\
\text { anzukämpfen. } \\
\text { Schulflucht nennt er es, wenn Eltern ihre } \\
\text { Kinder außerhalb ihrer Wohnbezirke } \\
\text { einschulen, . . . } \\
\text { Wer in ihrer Klasse das Problemkind ist, } \\
\text { weiß man nach zehn Minuten Unterricht. }\end{array}$ \\
\hline TÄTIGKEIT & $\begin{array}{l}\text {,die eigenaktive (+CONTR) } \\
\text { und dynamische (+DYN) } \\
\text { semantische Rolle eines } \\
\text { intransitiven Prädikats‘ }\end{array}$ & $\begin{array}{l}\text { John schrie. } \\
\text { Er kam nach Hause. } \\
\text { Wir gingen den Bach entlang. } \\
\text { Der Lärm machte den Kindern Angst. }\end{array}$ \\
\hline TÄTIGKEITSTRÄGER & $\begin{array}{l}\text {,gegenstandsbezogene } \\
\text { semantische Rolle des } \\
\text { Subjekts eines } \\
\text { Tätigkeitsprädikats‘ }\end{array}$ & $\begin{array}{l}\text { John schrie. } \\
\text { Er kam nach Hause. } \\
\text { Ich glaube/helfe/vertraue dir. } \\
\text { Der Lärm machte den Kindern Angst. }\end{array}$ \\
\hline TÄTIGKEITSBETROFFENER & $\begin{array}{l}\text {,gegenstandsbezogene } \\
\text { semantische Rolle des } \\
\text { Dativobjekts eines } \\
\text { Tätigkeitsprädikats‘ }\end{array}$ & $\begin{array}{l}\text { Ich glaube/helfe/vertraue dir. } \\
\text { Der Lärm machte den Kindern Angst. }\end{array}$ \\
\hline VORGANG & $\begin{array}{l}\text {,die nicht eigenaktive } \\
\text { (-CONTR) und dynamische } \\
\text { (+DYN) semantische Rolle } \\
\text { eines intransitiven } \\
\text { Prädikats‘ }\end{array}$ & $\begin{array}{l}\text { Die Statue schmolz. } \\
\text { Das Rad dreht sich. } \\
\text { Das Rad wird gedreht. }\end{array}$ \\
\hline VORGANGSTRÄGER & $\begin{array}{l}\text {,gegenstandsbezogene } \\
\text { semantische Rolle des } \\
\text { Subjekts eines } \\
\text { Vorgangsprädikats، }\end{array}$ & $\begin{array}{l}\text { Die Statue schmolz. } \\
\text { Das Rad dreht sich. } \\
\text { Das Rad wird gedreht. }\end{array}$ \\
\hline VORGANGSAUSLÖSER & $\begin{array}{l}\text {,gegenstandsbezogene } \\
\text { semantische Rolle des } \\
\text { Präpositional } \\
\text { einen+DAT-objekts }\end{array}$ & $\begin{array}{l}\text { Ein Junge wird von zwei Jugendlichen } \\
\text { geschlagen. }\end{array}$ \\
\hline
\end{tabular}


Tab. 2a (fortgesetzt)

\begin{tabular}{|c|c|c|}
\hline Rollenbezeichnung & Paraphrase & Beispiele \\
\hline VORGANGSBETROFFENER & $\begin{array}{l}\text {,gegenstandsbezogene } \\
\text { semantische Rolle des } \\
\text { Dativobjekts eines } \\
\text { Vorgangsprädikats، }\end{array}$ & Die Statue wird der Stadt gestiftet. \\
\hline VORGANGSSACHVERHALT & $\begin{array}{l}\text {,sachverhaltsbezogene } \\
\text { semantische Rolle des } \\
\text { Subjekts eines } \\
\text { Vorgangsprädikats، }\end{array}$ & $\begin{array}{l}\text { Dass es auch Familien gibt, wird } \\
\text { vergessen. } \\
\text { Für manch eine Mutter ist es schon } \\
\text { schwer genug auszuhalten, wenn ihr } \\
\text { sechsjähriges Kind mittags nach Hause } \\
\text { kommt... }\end{array}$ \\
\hline ZUSTAND & $\begin{array}{l}\text {,die nicht eigenaktive } \\
\text { (-CONTR) und statische } \\
\text { (-DYN) semantische Rolle } \\
\text { eines intransitiven } \\
\text { Prädikats، }\end{array}$ & $\begin{array}{l}\text { Dass Ylva nun direkt um die Ecke } \\
\text { eingeschult wurde, ist alles andere als } \\
\text { selbstverständlich, . . . } \\
\text { Wasser besteht aus Wasserstoff und } \\
\text { Sauerstoff. } \\
\text { Die Uhr gehört dem Juwelier. }\end{array}$ \\
\hline ZUSTANDSTRÄGER & $\begin{array}{l}\text {,gegenstandsbezogene } \\
\text { semantische Rolle des } \\
\text { Subjekts eines } \\
\text { Zustandsprädikats، }\end{array}$ & $\begin{array}{l}\text { Wasser besteht aus Wasserstoff und } \\
\text { Sauerstoff. } \\
\text { Die Uhr gehört dem Juwelier. }\end{array}$ \\
\hline ZUSTANDSBETROFFENER & $\begin{array}{l}\text {,gegenstandsbezogene } \\
\text { semantische Rolle des } \\
\text { Dativobjekts eines } \\
\text { Zustandsprädikats‘ }\end{array}$ & Die Uhr gehört dem Juwelier. \\
\hline ZUSTANDSSACHVERHALT & $\begin{array}{l}\text {,sachverhaltsbezogene } \\
\text { semantische Rolle des } \\
\text { Subjekts eines } \\
\text { Zustandsprädikats، }\end{array}$ & $\begin{array}{l}\text { Dass Ylva nun direkt um die Ecke } \\
\text { eingeschult wurde, ist alles andere als } \\
\text { selbstverständlich, . . }\end{array}$ \\
\hline
\end{tabular}

Tab. 2b: Signifikativ-semantische Präpositionalrollen.

\begin{tabular}{lll}
\hline AdReSSATUm (an + Akk) & ,Zielgröße der Übergabe & $\begin{array}{l}\text { Er schreibt an seine } \\
\text { Schwester. }\end{array}$ \\
\hline AfFIZIERT (an + Dat) & ,partiell Erfasstes & $\begin{array}{l}\text { Der Hund nagt an seinem } \\
\text { Knochen. }\end{array}$ \\
\hline DeteCtUM (an + Dat) & ,Maßgröße der Klassifikation“ & $\begin{array}{l}\text { Das Label misst den Erfolg } \\
\text { an Verkaufszahlen. }\end{array}$ \\
\hline
\end{tabular}


Tab. 2b (fortgesetzt)

\begin{tabular}{|c|c|c|}
\hline PROSPECTUM (auf + Akk) & ,zukünftiges Ereignis‘ & Er freut sich auf den Urlaub. \\
\hline DESTINATUM (auf + Akk) & ,Fokuspunkt der Aufmerksamkeit‘ & Er achtet auf die Kinder. \\
\hline BASIS (auf + Akk) & ,Grundlage des Szenarios“ & $\begin{array}{l}\text { Sie baut auf ihre } \\
\text { Mitarbeiter. }\end{array}$ \\
\hline BASIS (auf + Dat) & ,Grundlage des Szenarios“ & $\begin{array}{l}\text { Das Geheimnis lastet auf } \\
\text { seiner Frau. }\end{array}$ \\
\hline MATERIAL (aus + Dat) & ,Grundstoff“ & $\begin{array}{l}\text { Er bastelt ein Flugzeug aus } \\
\text { Pappe. }\end{array}$ \\
\hline BENEFACTUM (für + Akk) & ,Nutznießer‘ & $\begin{array}{l}\text { Wir haben alles für dich } \\
\text { getan. } \\
\text { Ich kaufe Blumen für dich. }\end{array}$ \\
\hline OFFICIUM (für + Akk) & $\begin{array}{l}\text {,der Gegenleistung zugrundeliegende } \\
\text { Leistung، }\end{array}$ & $\begin{array}{l}\text { Er bestraft die Kinder für } \\
\text { ihr Verhalten. }\end{array}$ \\
\hline $\begin{array}{l}\text { OPPONENTUM (gegen + } \\
\text { Akk) }\end{array}$ & ,Gegengröße/Antipode“ & $\begin{array}{l}\text { Er wendet sich gegen die } \\
\text { Regierung. }\end{array}$ \\
\hline $\begin{array}{l}\text { TRANSFORMATUM }(\text { in }+ \\
\text { Akk) }\end{array}$ & $\begin{array}{l}\text {,Ergebnis einer erfolgten Umwandlung/ } \\
\text { Metamorphose“ }\end{array}$ & Er verwandelt Blei in Gold. \\
\hline DISZIPLIN (in + Dat) & $\begin{array}{l}\text {,abstrakter, im weiteren Sinn der } \\
\text { Entwicklung zugehöriger } \\
\text { Betätigungsraum‘ }\end{array}$ & Er übt in Mathe. \\
\hline RESPONS (in + Dat) & ,das Erkannte‘ & $\begin{array}{l}\text { Er erkennt seinen Bruder in } \\
\text { dem Fremden. }\end{array}$ \\
\hline IDENTIFICATUM (mit + Dat) & ,hat die Eigenschaft‘ & $\begin{array}{l}\text { Mit der Platte nimmt er ein } \\
\text { Meisterwerk auf. }\end{array}$ \\
\hline QUAESITUM (nach + Dat) & ,das Gesuchte، & Er sucht nach Fehlern. \\
\hline $\begin{array}{l}\text { KRITERIUM (nach + Dat) } \\
\text { INTELLEKTUELLES THEMA } \\
(u ̈ b e r+\text { Akk })\end{array}$ & $\begin{array}{l}\text { „Maßgröße‘ } \\
\text {,inhaltlicher Gegenstand der } \\
\text { intellektuellen Aktivität‘ }\end{array}$ & $\begin{array}{l}\text { Er urteilt nach dem Schein. } \\
\text { Er diskutiert über Goethe. }\end{array}$ \\
\hline INFERIOR (über + Akk) & ,ein hierarchisch Niedrigeres“ & $\begin{array}{l}\text { Der König herrscht über } \\
\text { seine Untertanen. }\end{array}$ \\
\hline OKKUPATION (über + Dat) & $\begin{array}{l}\text {,intensive, einnehmende und leicht } \\
\text { negativ konnotierte Aktivität‘ }\end{array}$ & $\begin{array}{l}\text { Sie vergisst die Arbeit über } \\
\text { dem Erzählen. }\end{array}$ \\
\hline ACCIPIENDUM (um + Akk) & ,ein zu Erhaltendes‘ & Sie kämpfen um den Pokal. \\
\hline SEPARATUM (von + Dat) & ,das abstrakt Entnommene“ & Sie trennt sich von ihm. \\
\hline
\end{tabular}


Tab. 2b (fortgesetzt)

\begin{tabular}{lll}
\hline $\begin{array}{l}\text { PUNKTUELLES Thema } \\
\text { (von + Dat) }\end{array}$ & $\begin{array}{l}\text {,inhaltlicher Gegenstand der } \\
\text { oberflächlichen Aktivität }\end{array}$ & Er faselt von Liebe. \\
\hline SWARM (von + Dat) & ,raumfüllende Entität & $\begin{array}{l}\text { Der Garten wimmelt von } \\
\text { Bienen. }\end{array}$ \\
\hline MALUS (vor + Dat) & ,negativ attribuierte Entität & $\begin{array}{l}\text { Beide fliehen vor dem } \\
\text { hartnäckigen Killer. }\end{array}$ \\
\hline SWARM (vor + Dat) & ,raumfüllende Entität & $\begin{array}{l}\text { Der Garten wimmelt vor } \\
\text { Bienen. }\end{array}$ \\
\hline RESULTATUM (zu + Dat) & ,Ergebnis“ & Die Taktik verhilft zum Sieg. \\
\hline
\end{tabular}

\subsection{Vergleichende Textanalyse: denotativ- vs. signifikativ-semantisch}

Die grammatischen Grundstrukturen des Textes wurden mit Hilfe der in Kap. 4.1 vorgestellten Methode linear, d.h. entsprechend der Reihenfolge ihres Vorkommens, identifiziert und syntaktisch beschrieben. Die syntaktische Beschreibung (= SYN) orientiert sich dabei an den Satzgliedwerten der GTA (2017: 249-687). Anschließend erfolgte die semantische Analyse mit Hilfe der in Kap. 4.2 vorgestellten Rollensets: denotativ-semantisch (= DEN) vs. signifikativ-semantisch (= SIG).

Naturgemäß ist eine solche Textanalyse (s. Tab. 3) lang, und sicher kann man sie auch überblättern. Aus der Sicht unserer Argumentation ist jedoch die vergleichende Textanalyse zentral, da sie die theoretische Überlegenheit des signifikativ-semantischen Modells empirisch dokumentiert und die Schlüsse, die in Kap. 5.1 gezogen werden, begründet. Außerdem bildet sie die empirische Grundlage für eine neue Satzbauplansystematik des Gegenwartsdeutschen (Kap. 5.2).

Um jedoch wenigstens die Präsentation der Textanalyse zu kürzen, wird die Analyse im Folgenden nicht linear, sondern gebündelt vorgestellt. Die Struktur dieser Bündelung sieht wie folgt aus: ${ }^{29}$

29 Bei der Planung der Struktur der Bündelung mussten wir feststellen, dass als Leitstrukturen nur die signifikativ-semantischen Grundstrukturen in Frage kommen. Denn bei den denotativsemantischen Grundstrukturen gibt es zahlreiche Lücken und Fragezeichen (siehe hierzu Kap. 5.1), während die syntaktischen Strukturbeschreibungen, die Komplemente wie Supplemente enthalten, viel zu facettenreich sind, um eine kürzere und übersichtlichere Präsentation zu ermöglichen. 


\begin{tabular}{l} 
signifikativ-semantische Grundstruktur Nr. 1 \\
der erste dazugehörige Beleg \\
syntaktische Grundstruktur des Belegs \\
\hline denotativ-semantische Grundstruktur des Belegs \\
\hline der zweite dazugehörige Beleg \\
\hline syntaktische Grundstruktur des Belegs \\
\hline denotativ-semantische Grundstruktur des Belegs \\
\hline$\ldots$ \\
\hline signifikativ-semantische Grundstruktur Nr. 2 \\
\hline der erste dazugehörige Beleg \\
\hline syntaktische Grundstruktur des Belegs \\
\hline denotativ-semantische Grundstruktur des Belegs \\
\hline$\ldots$ \\
\hline
\end{tabular}

\section{Abkürzungen}

\begin{tabular}{ll}
\hline ADV & $=$ Adverbial \\
\hline DIR & $=$ Direktivum \\
\hline kom & $=$ Komitativ(adverbial) \\
\hline KOMM & $=$ Mikrosubjekt (siehe Kap. 5.1) \\
\hline MIKROSUB & $=$ Objekt \\
\hline OBJ & $=$ Frädikat \\
PRÄD & $=$ Valenz- und Rollensimulation (siehe Kap. 5.1) \\
\hline PRÄDI & $=$ Subjekt \\
\hline simul & $=$ unterspezifiziert \\
\hline SUB & $=$ Vermittlung(sadverbial) (siehe [S135a]) \\
\hline unterspez & \\
\hline verm &
\end{tabular}




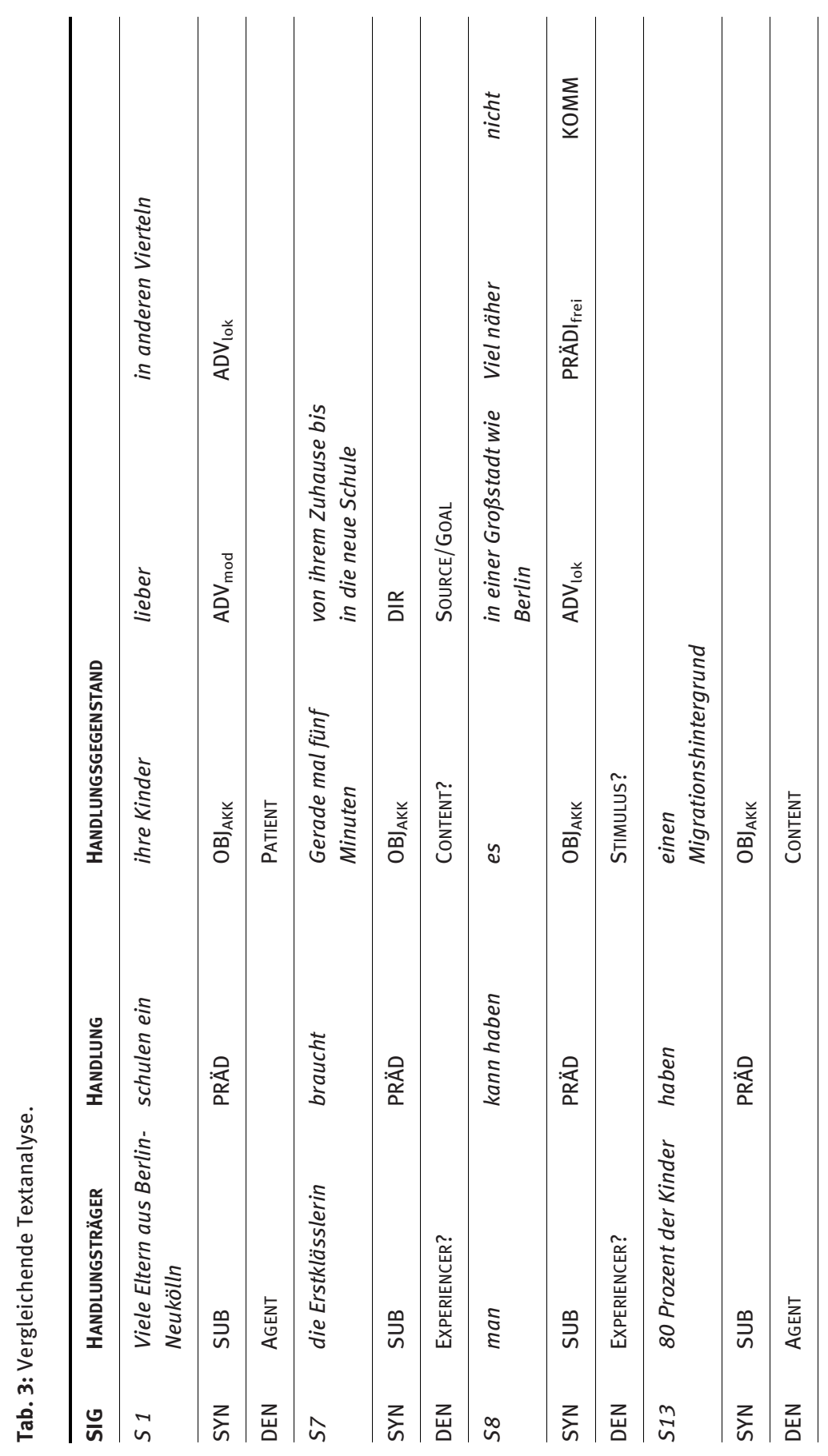




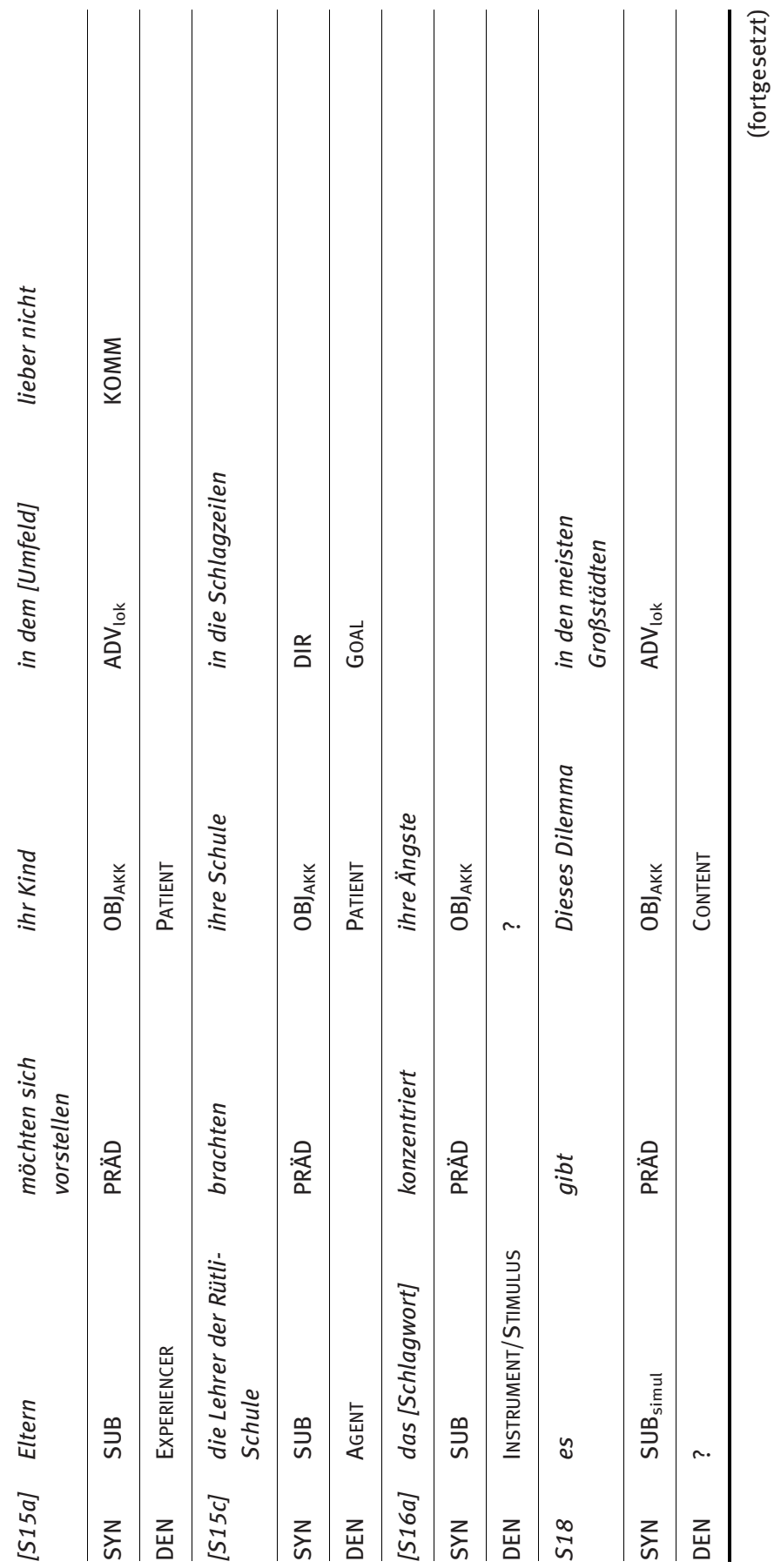




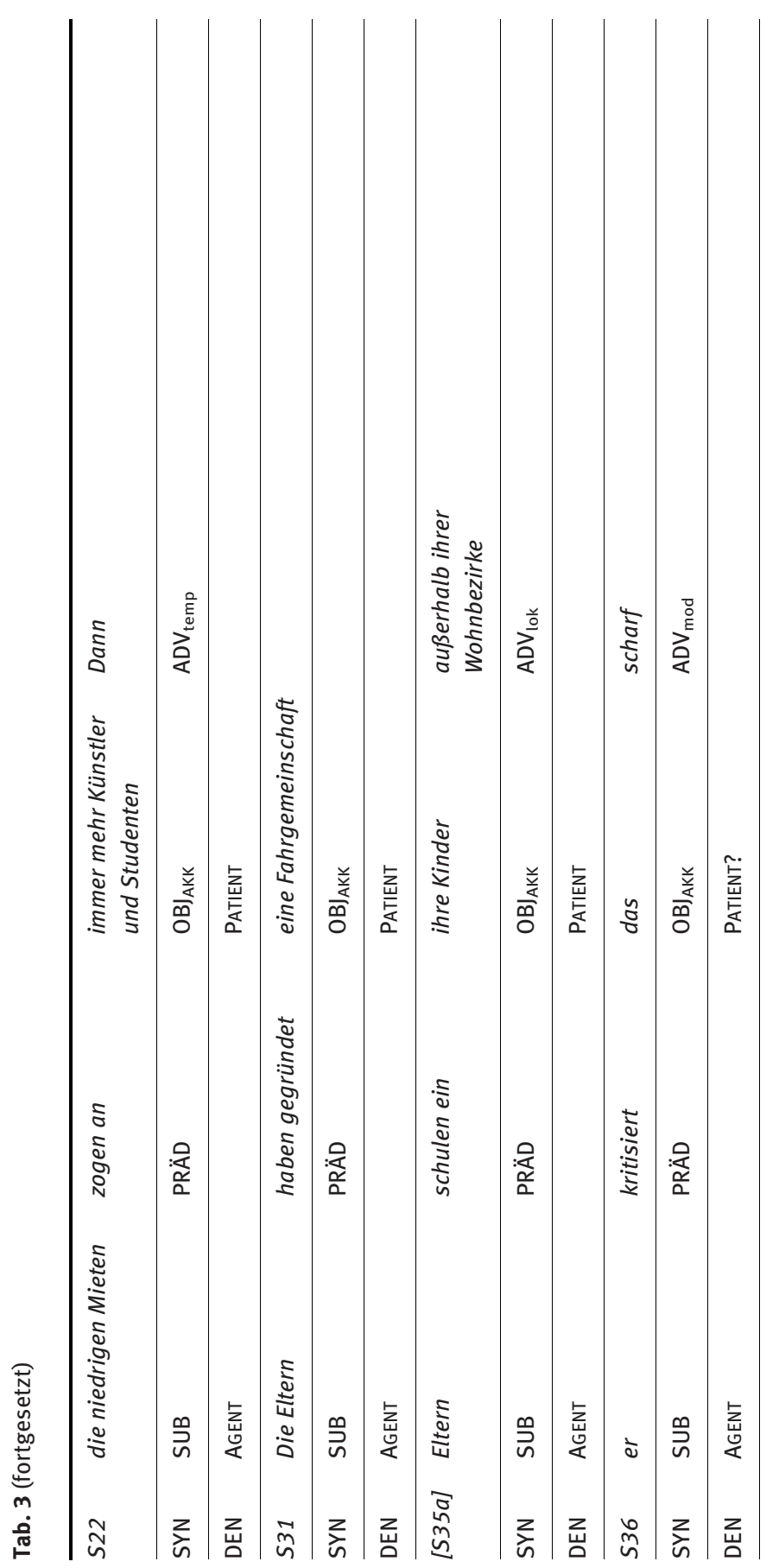




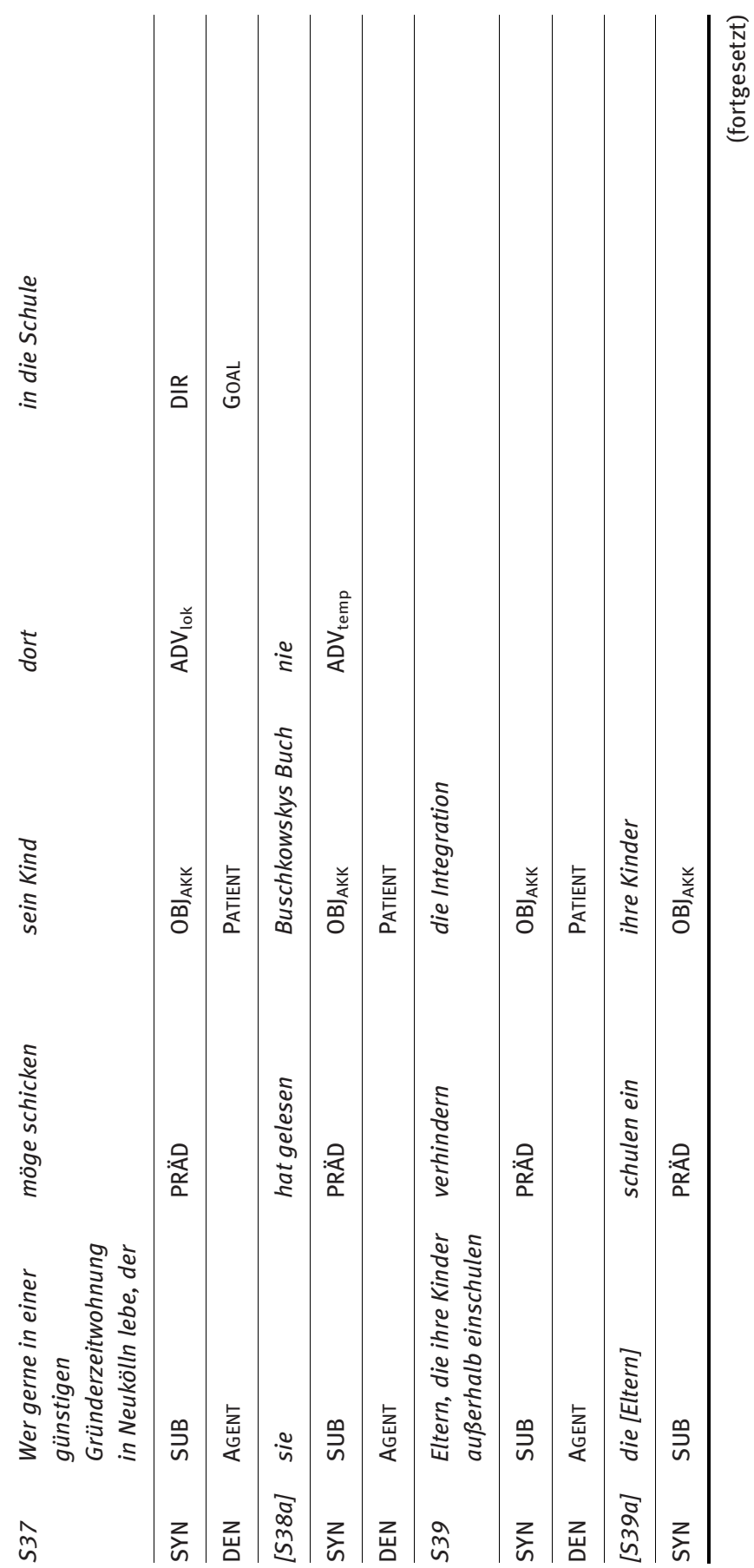




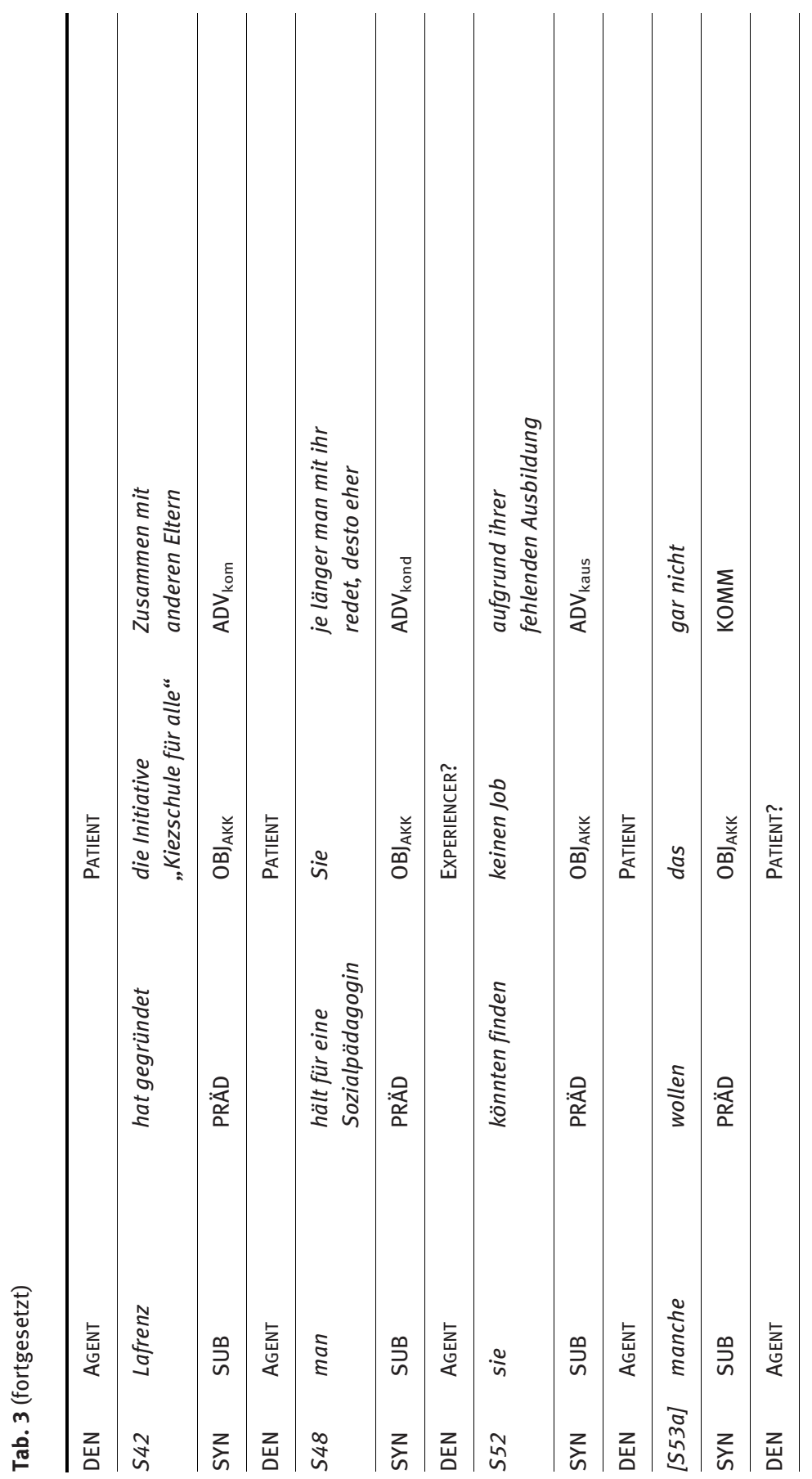




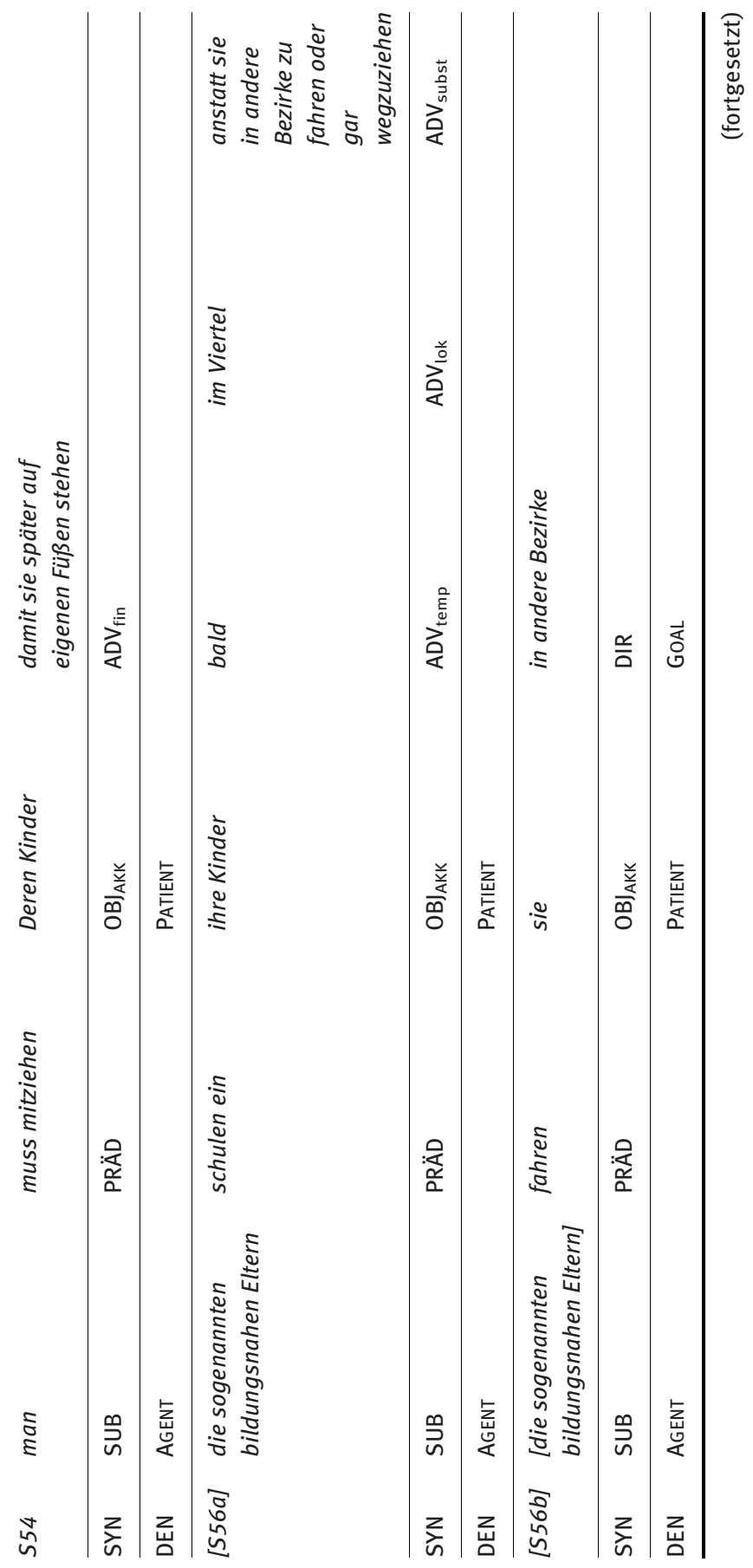




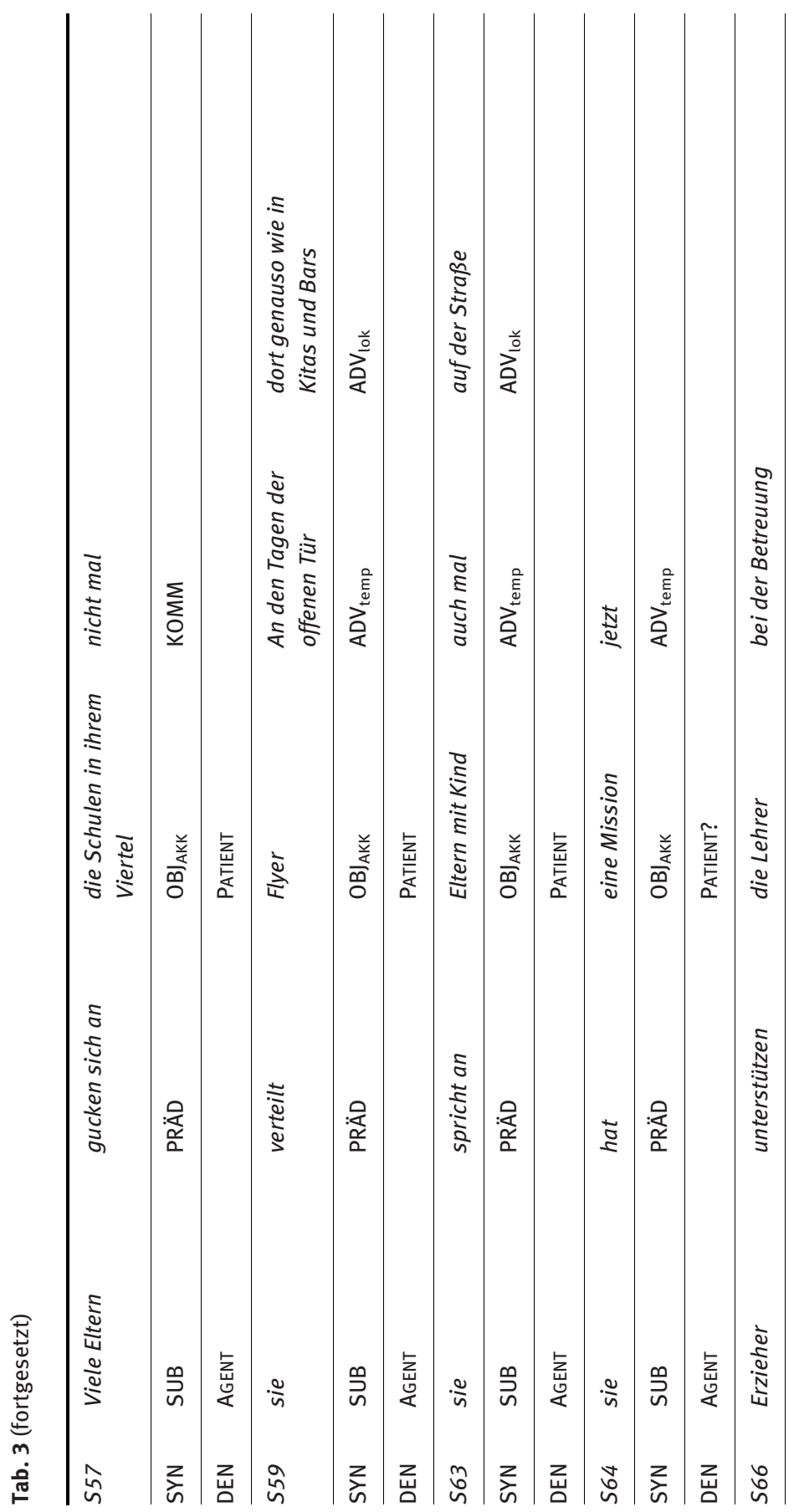




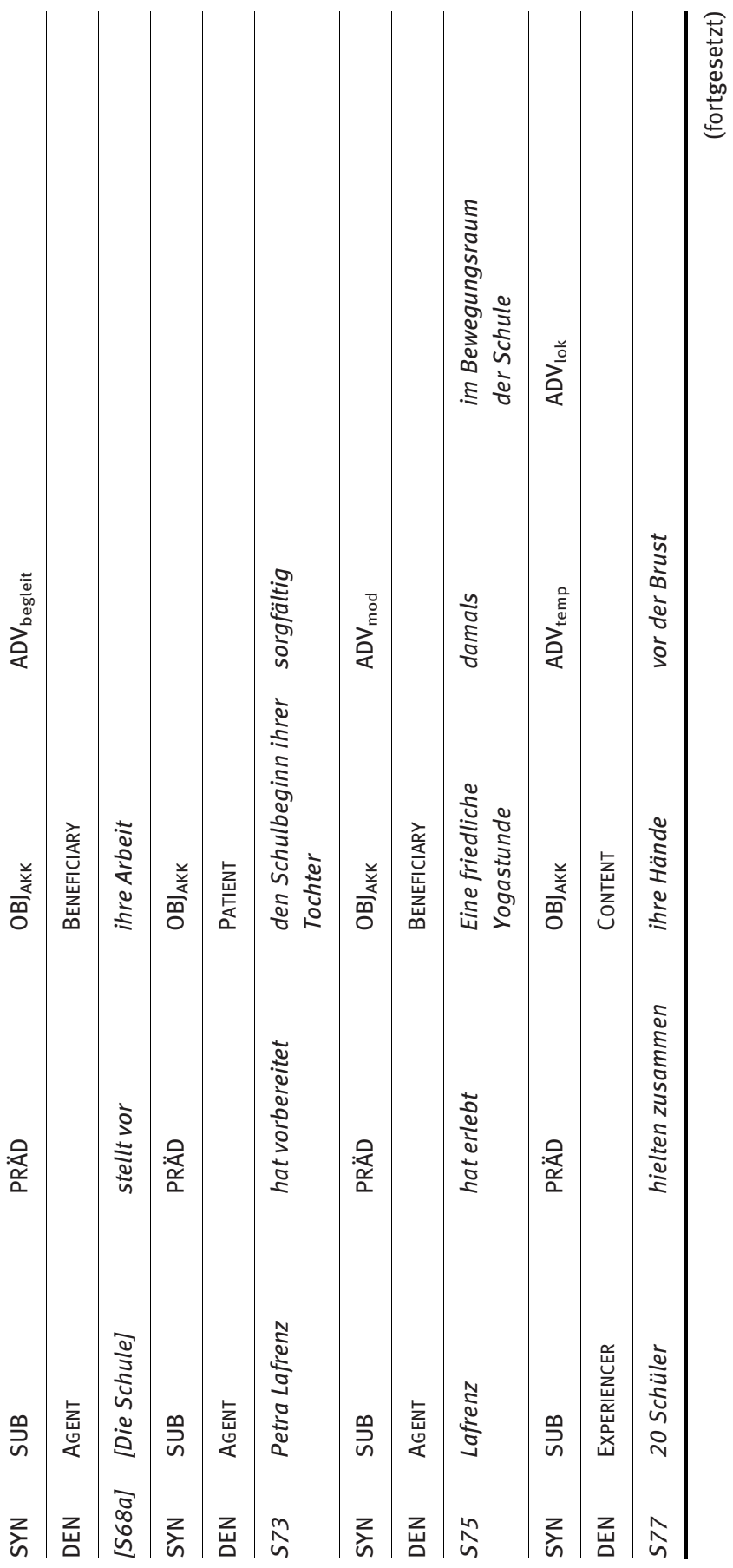




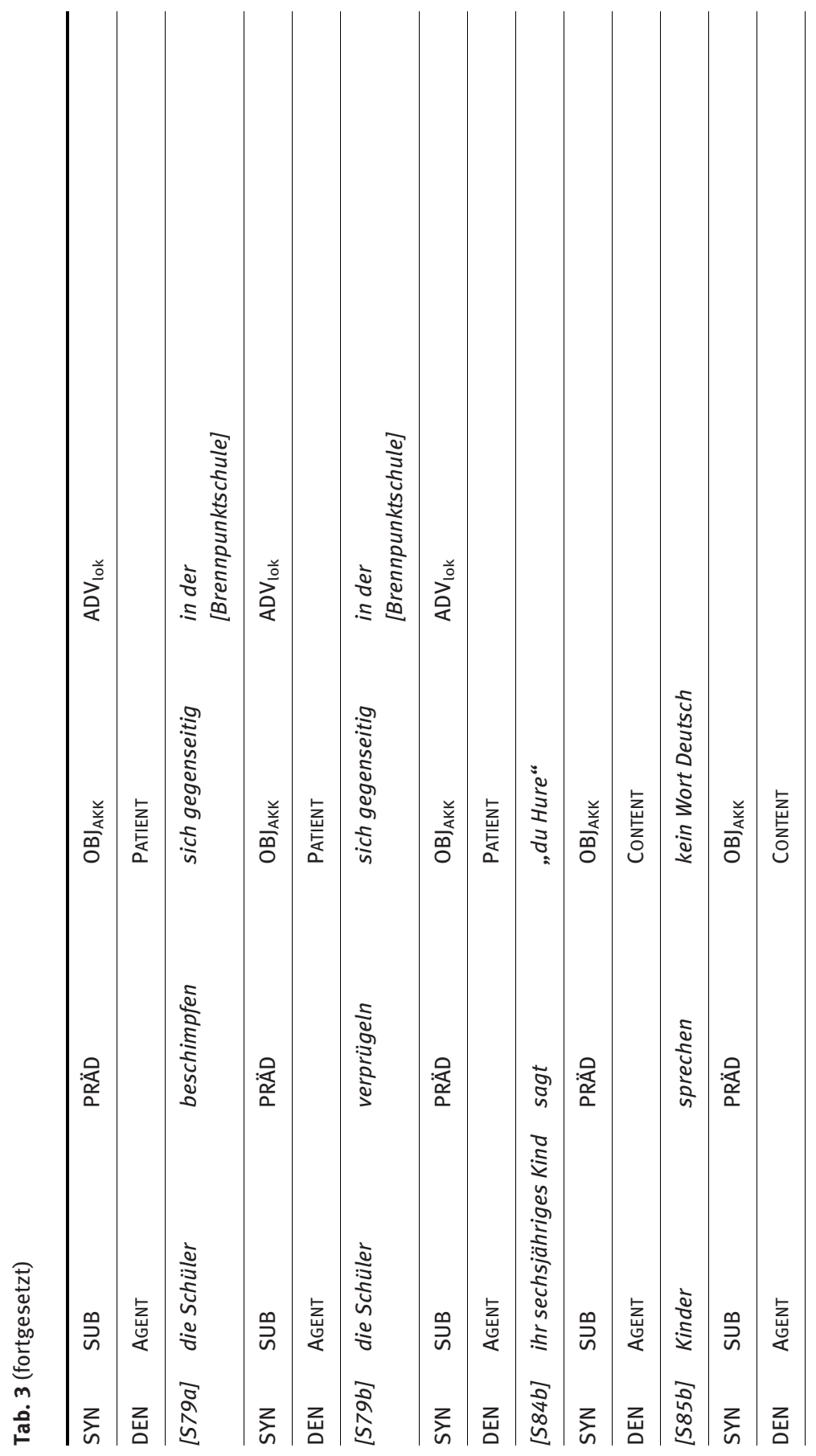




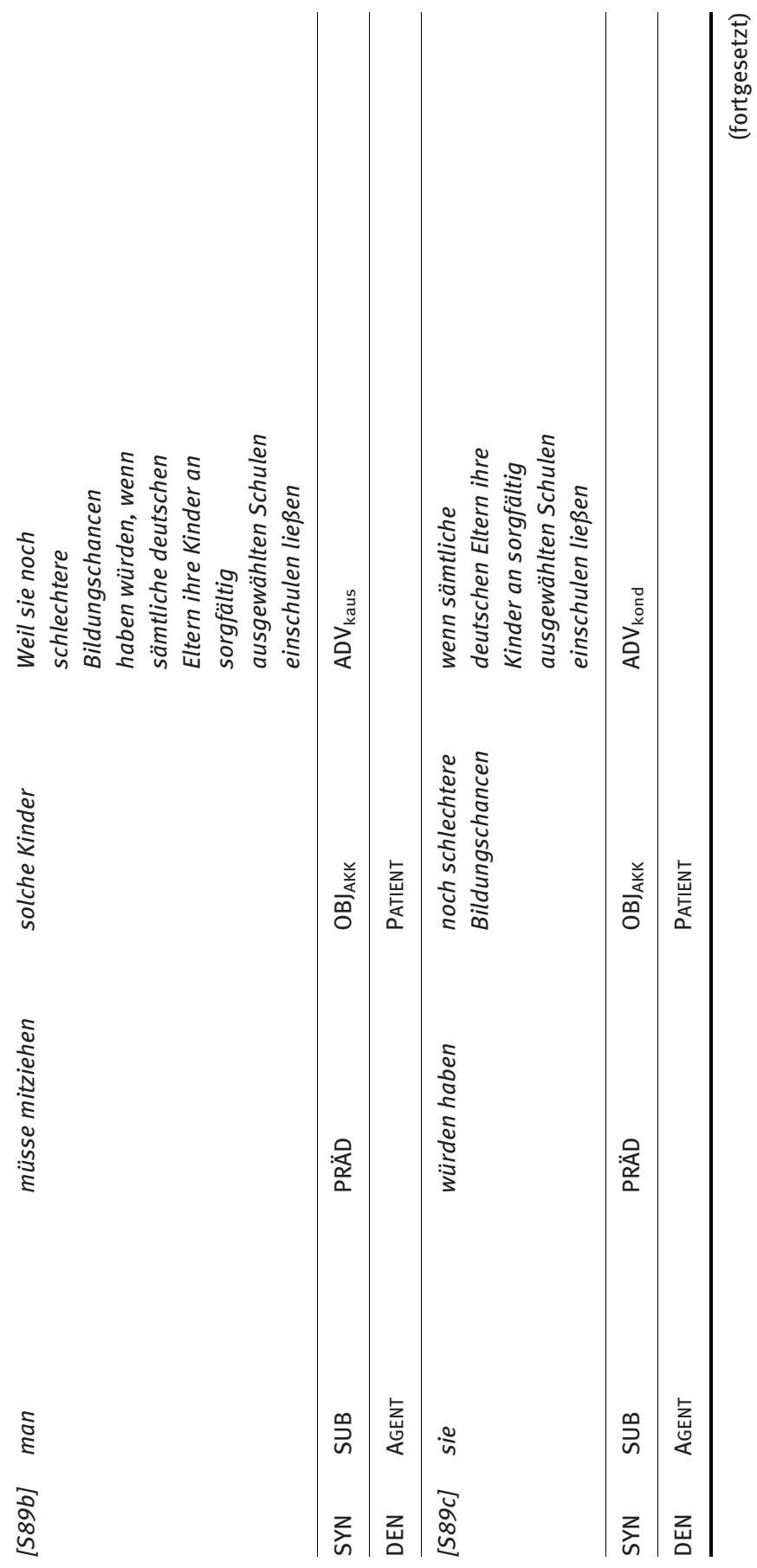




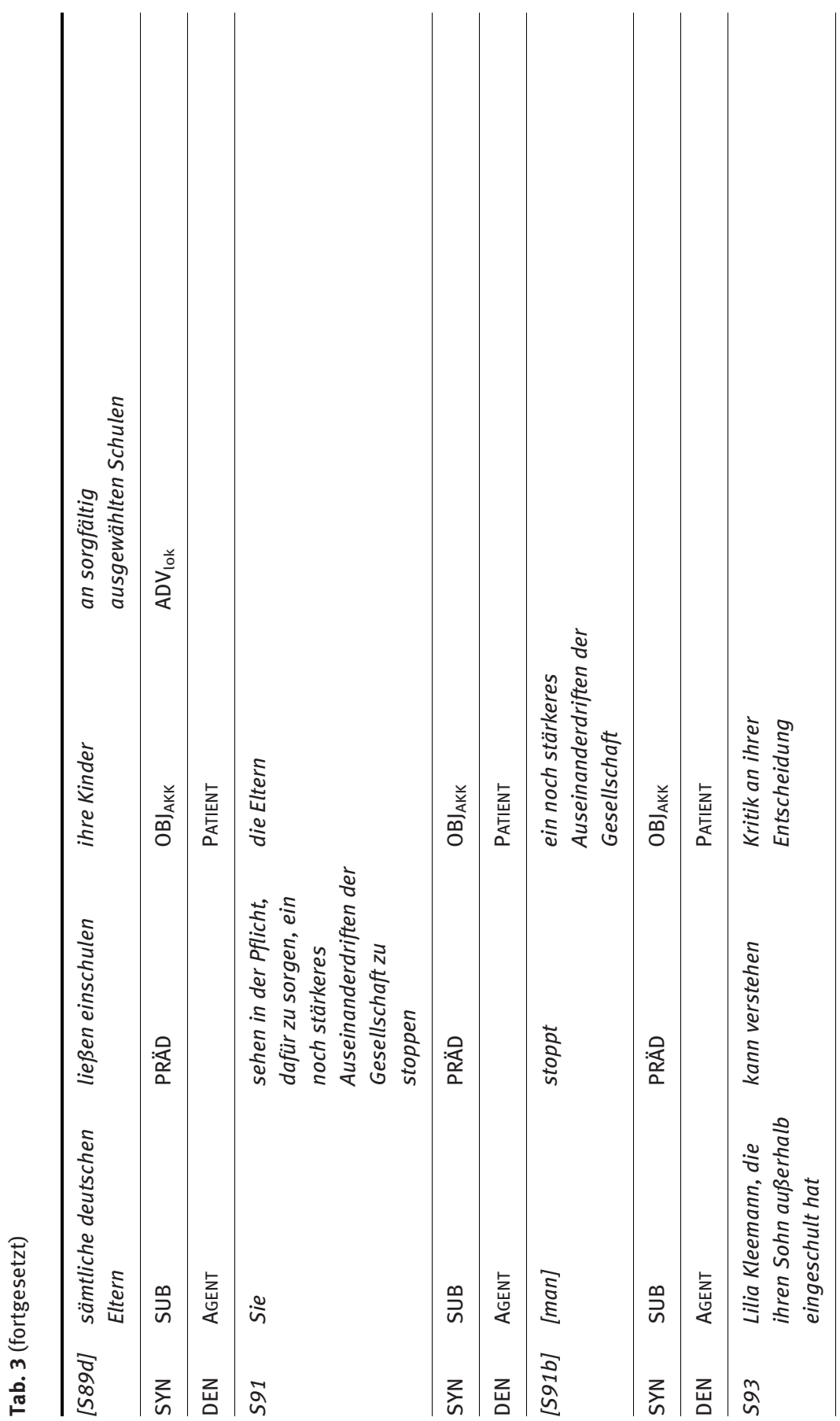




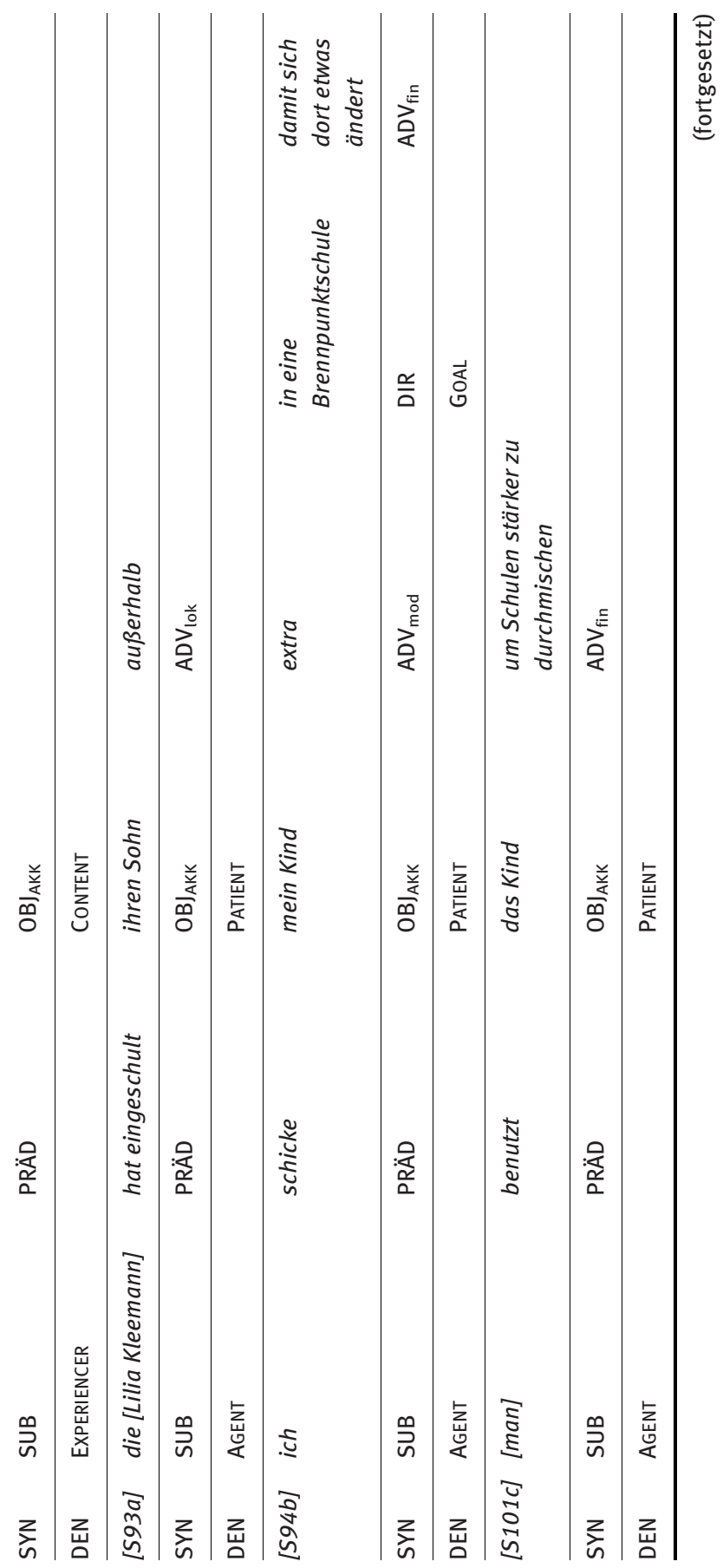




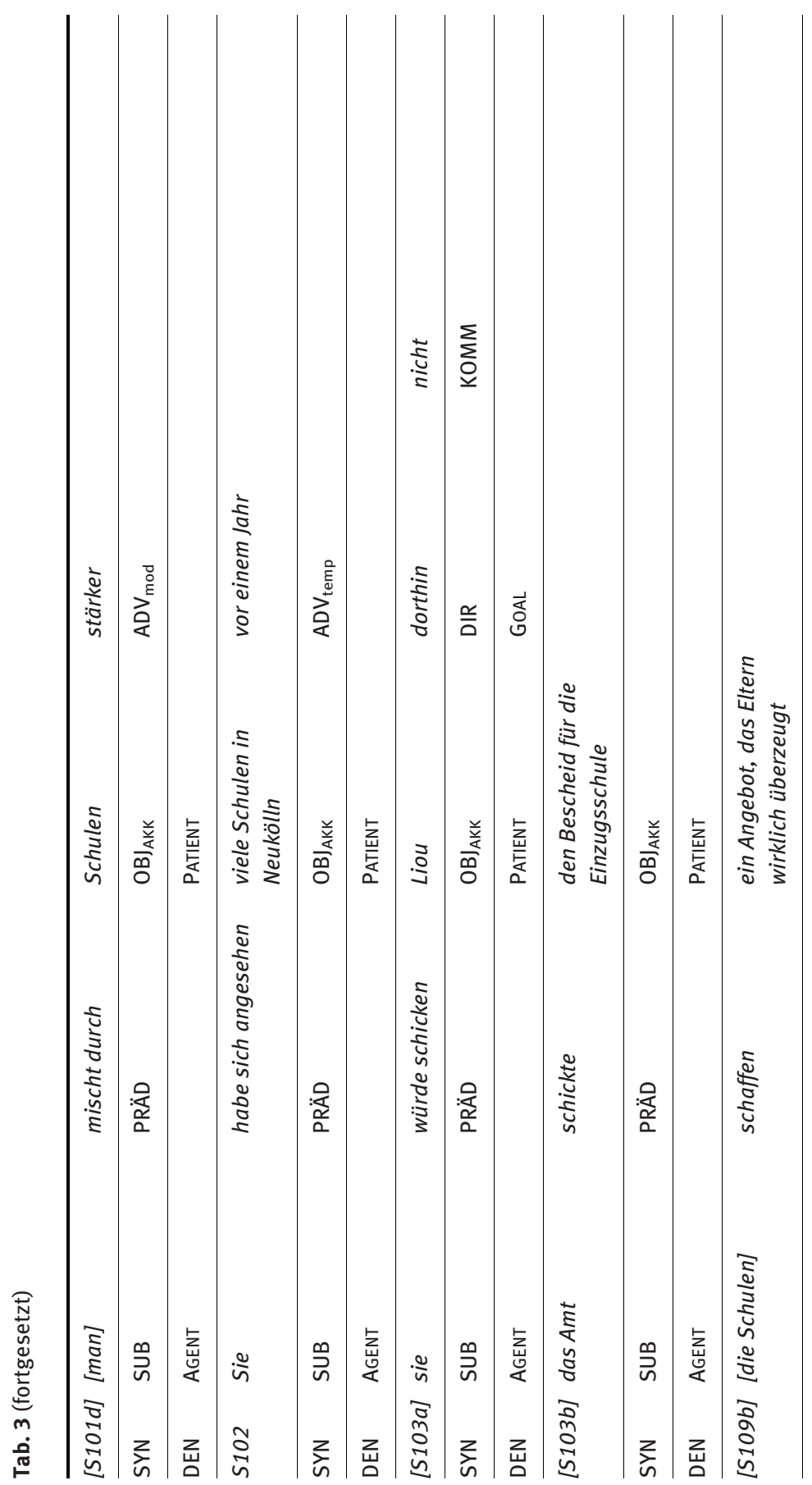




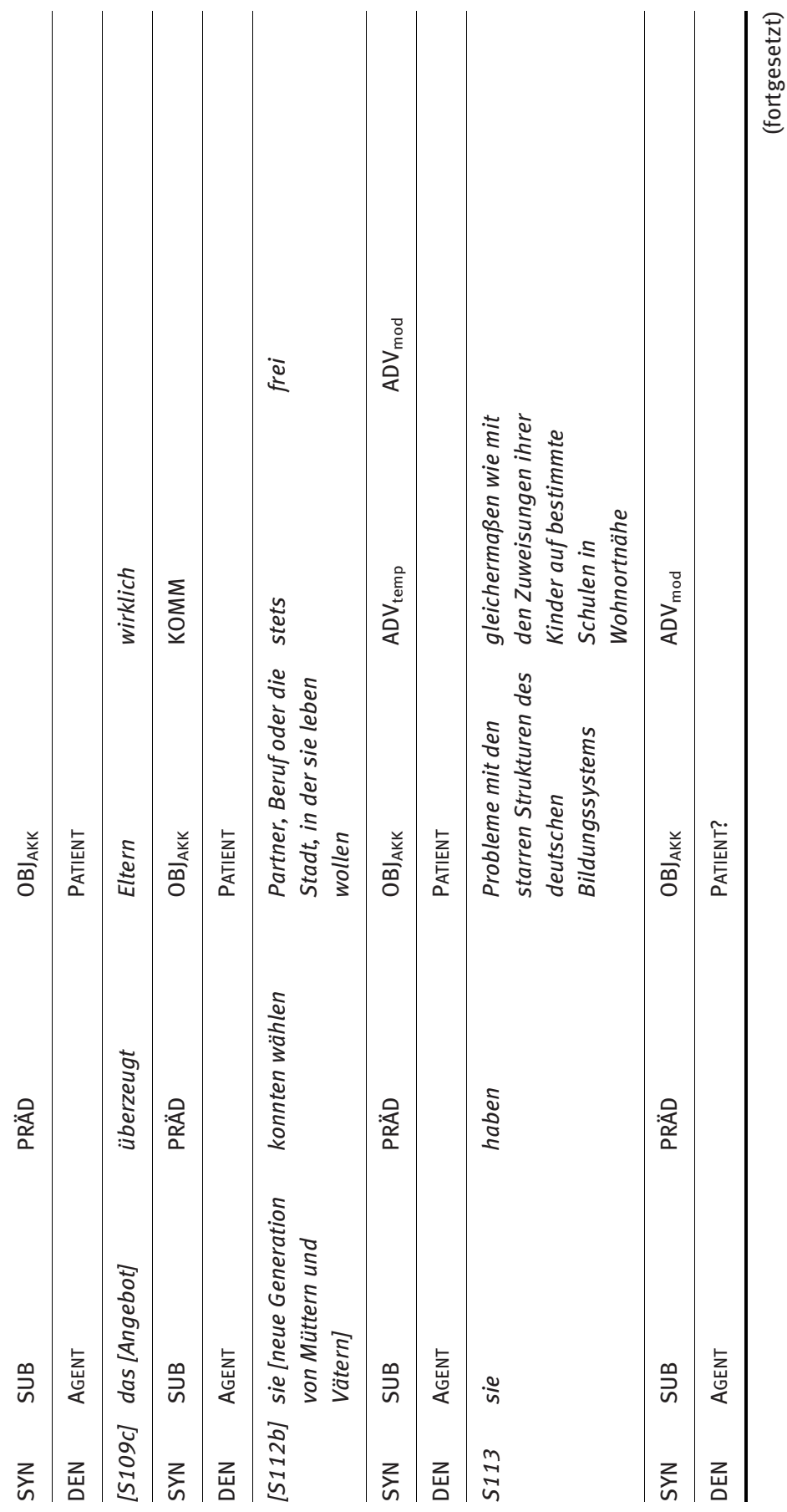




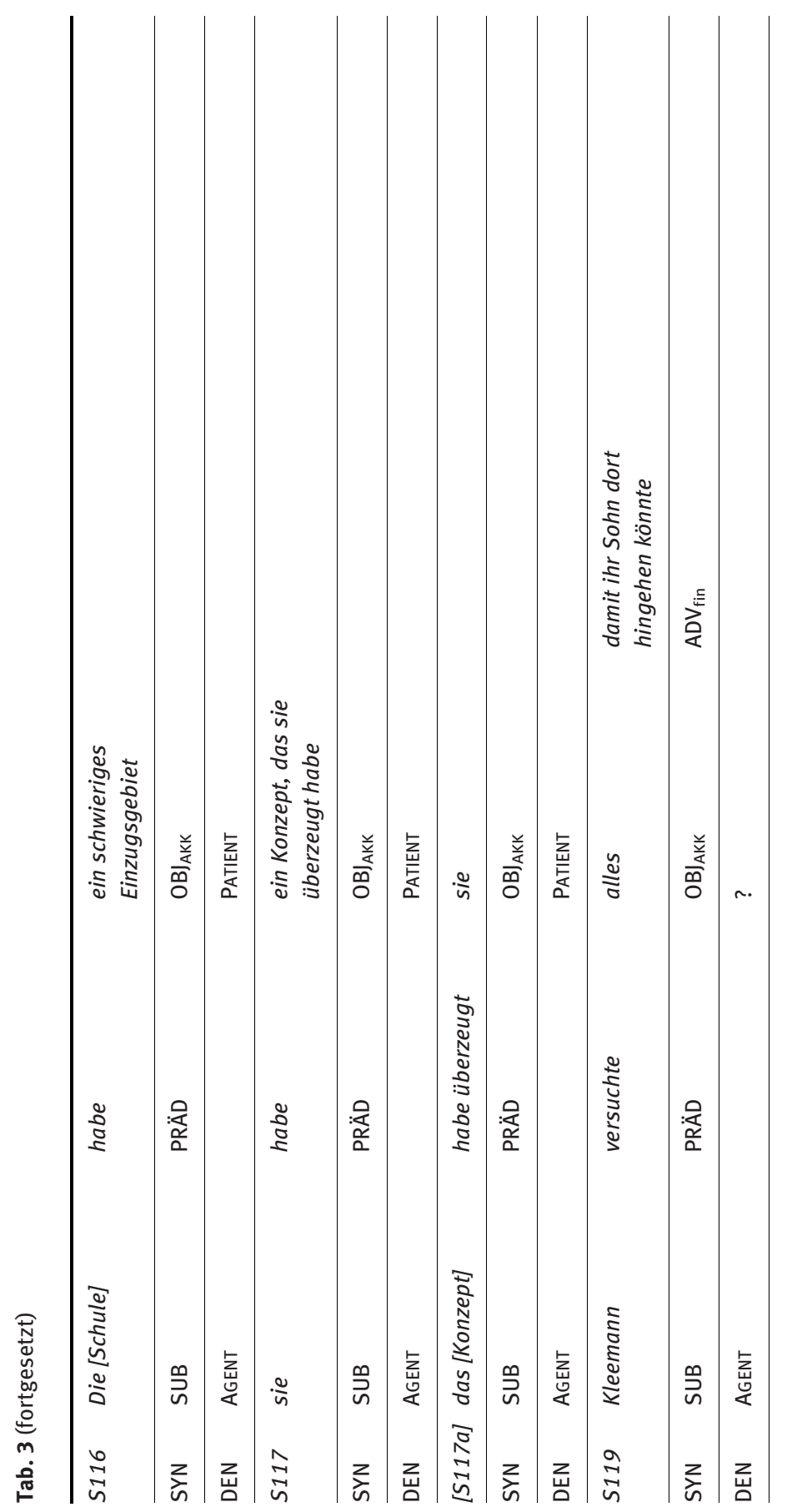




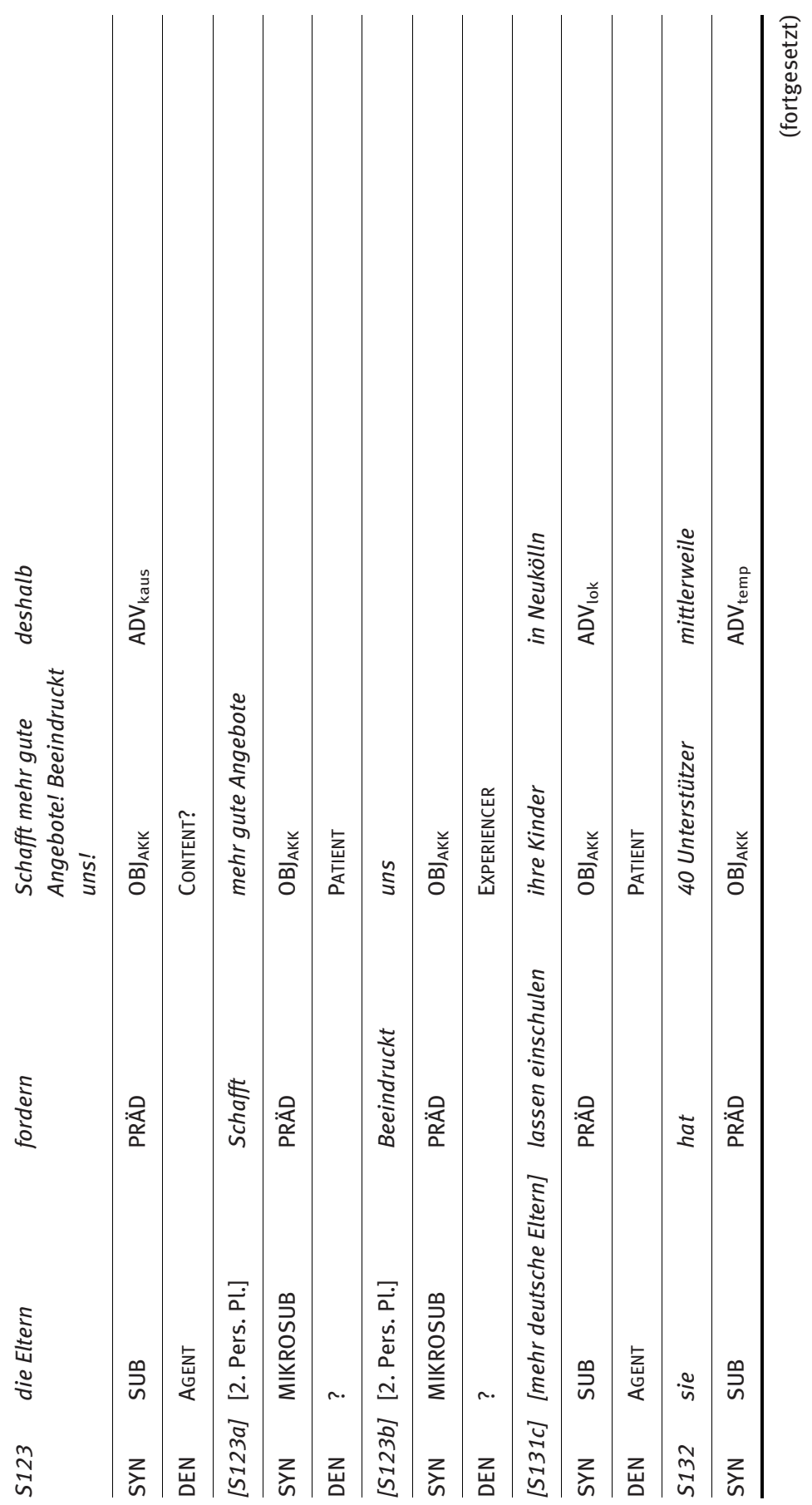




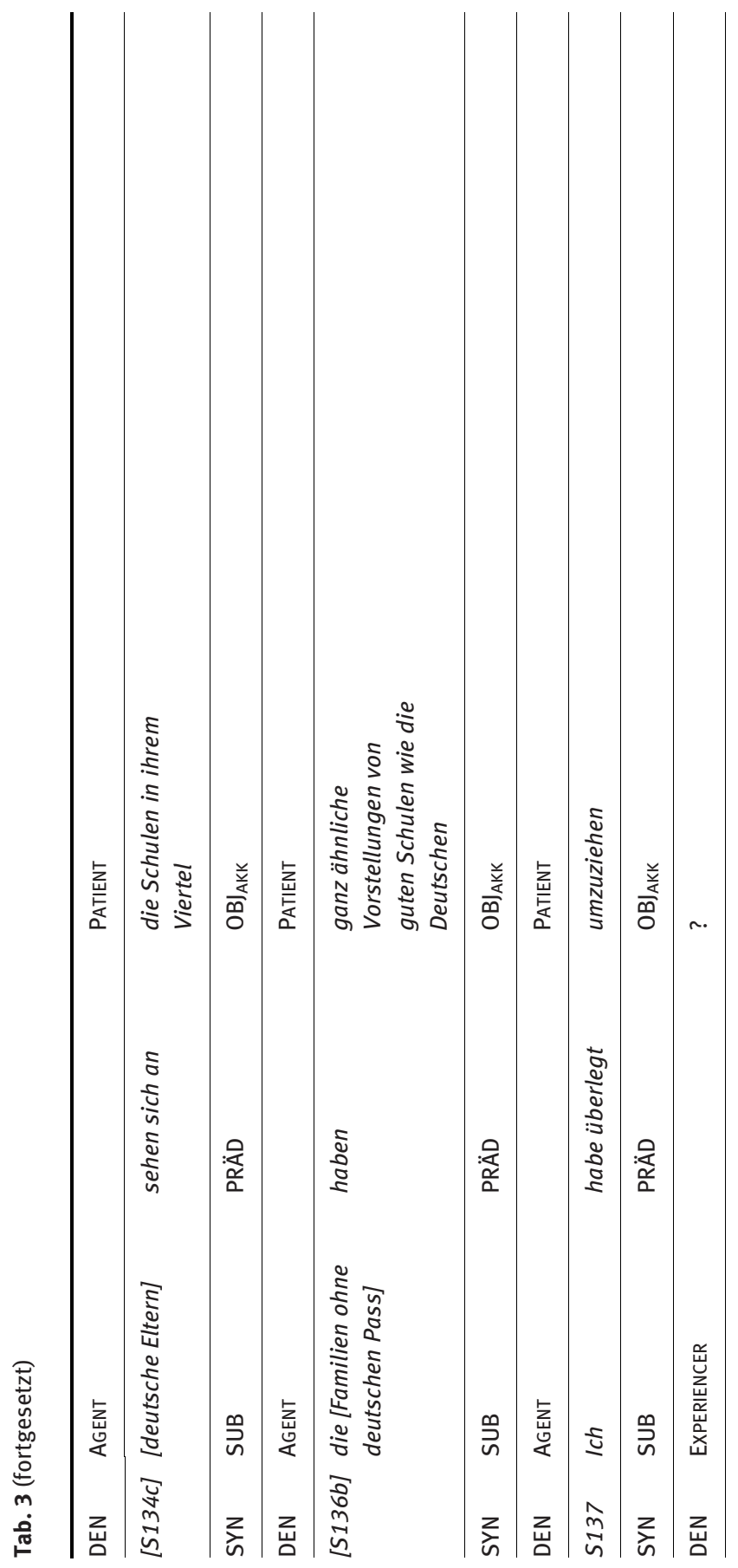




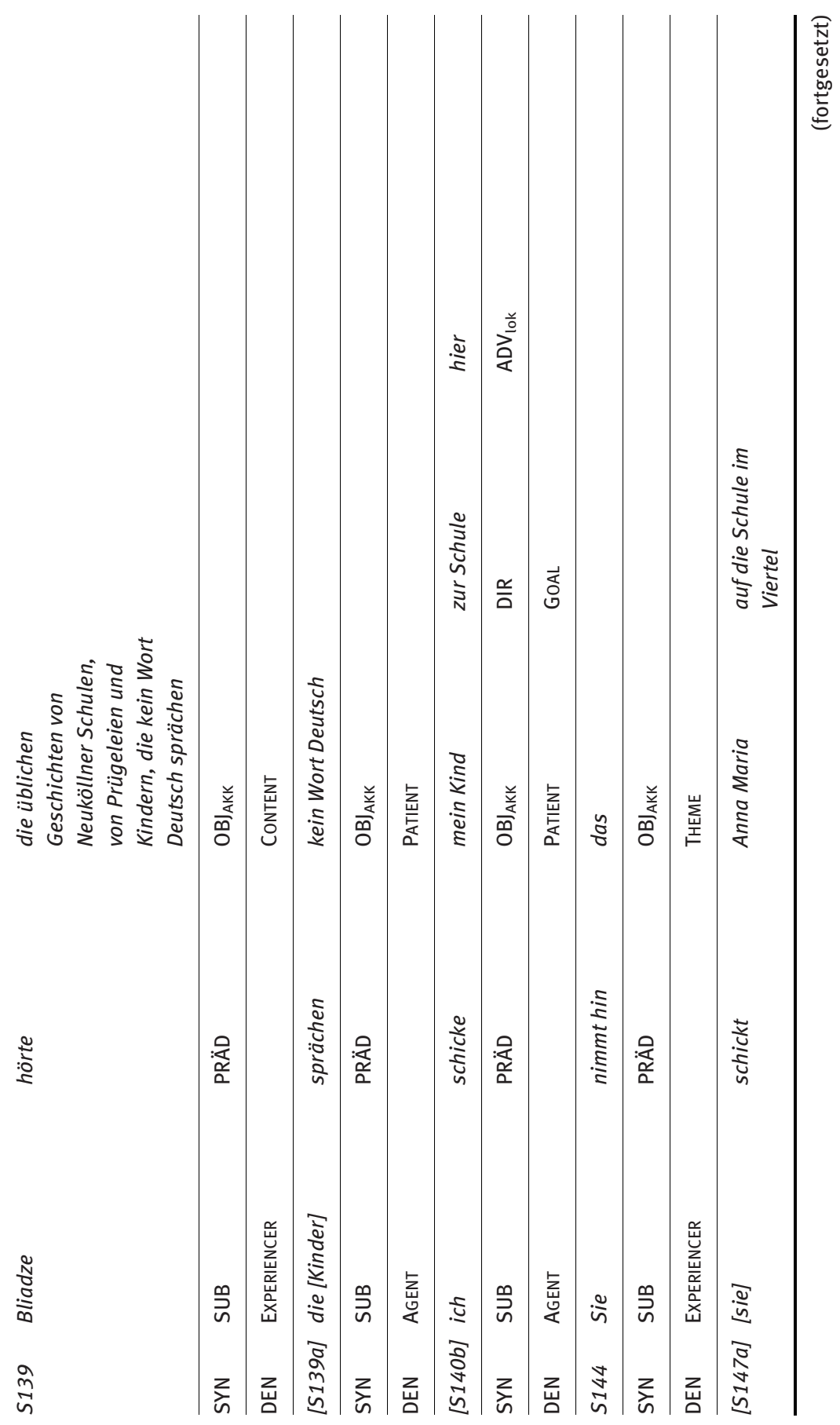




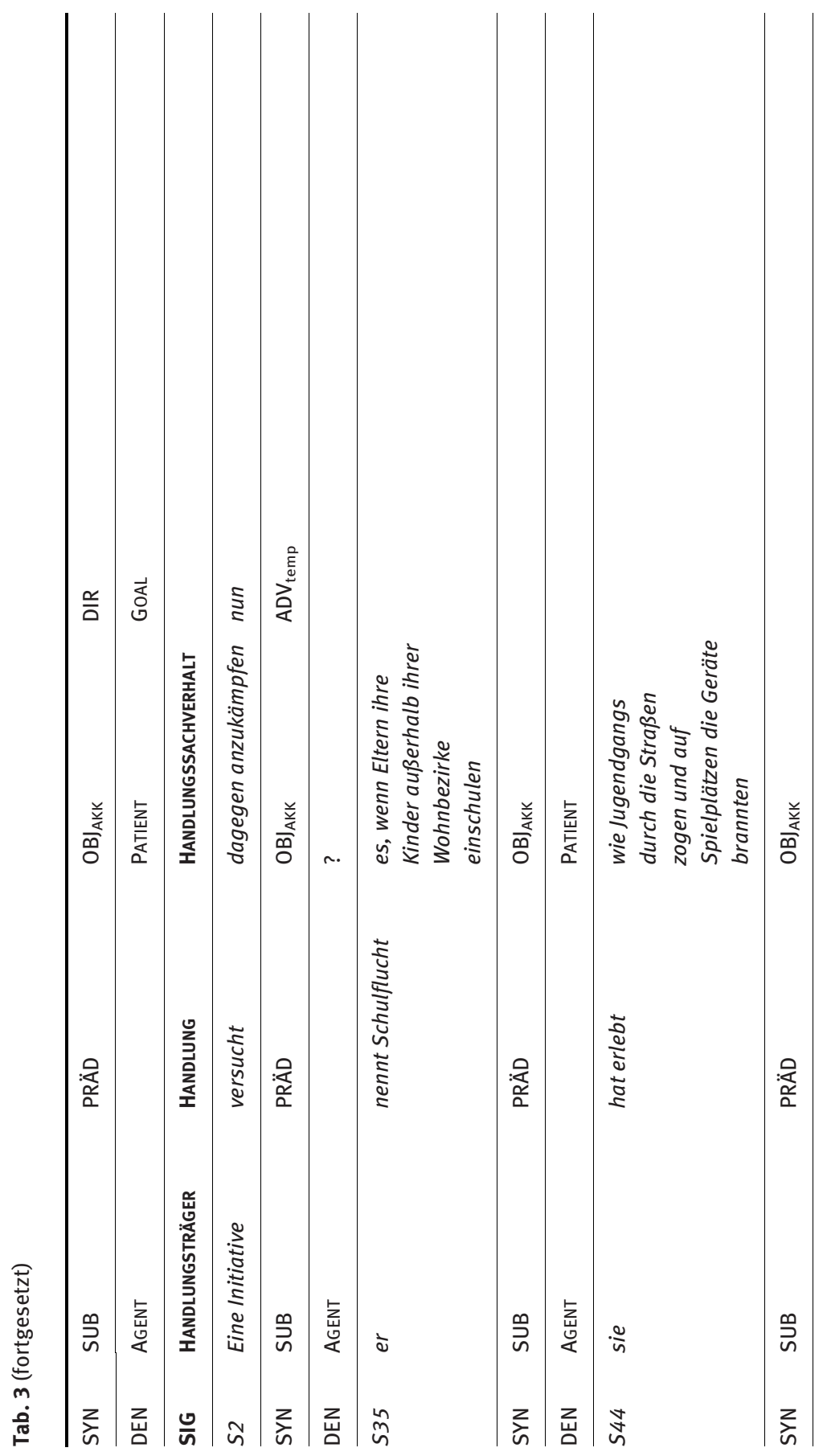




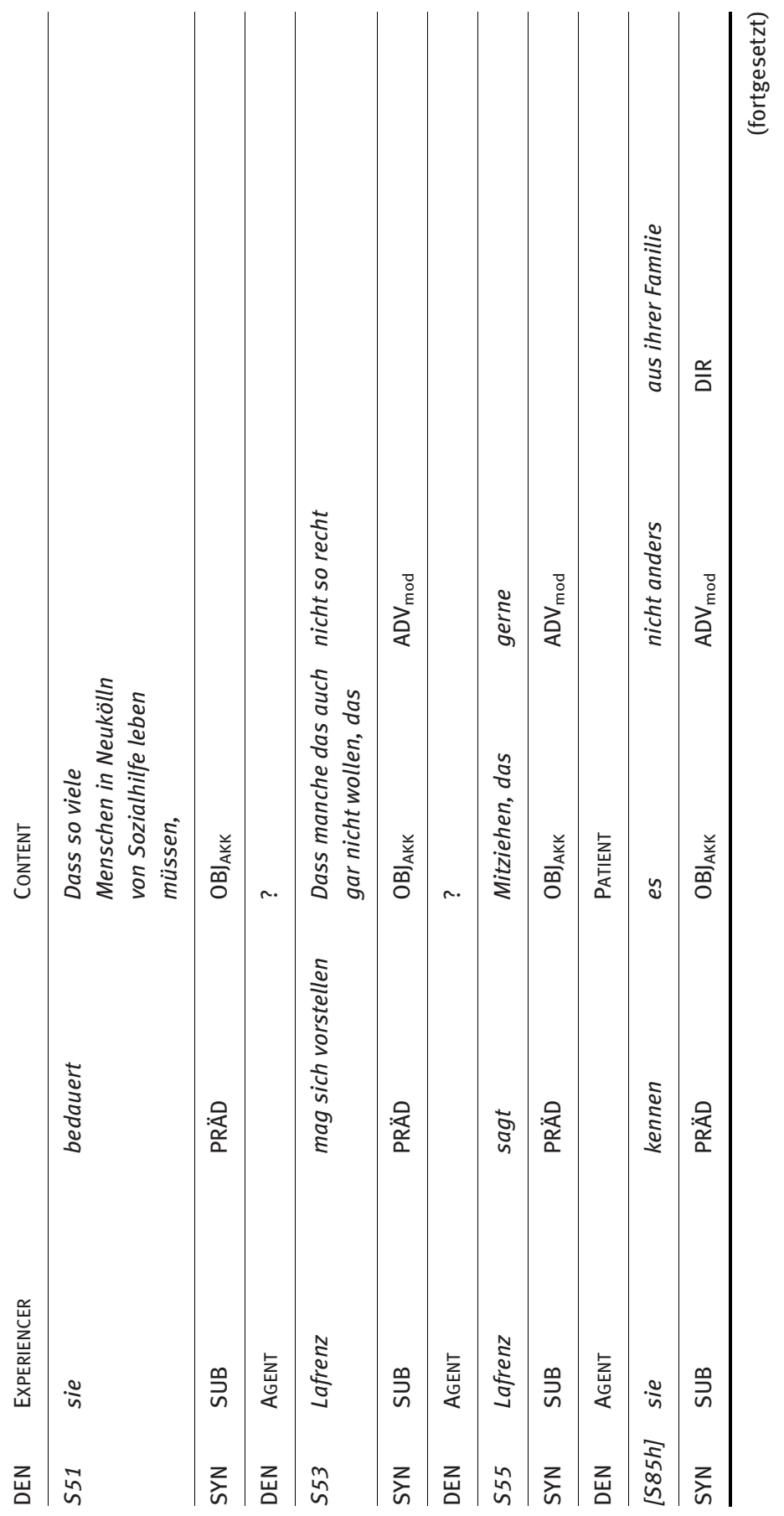




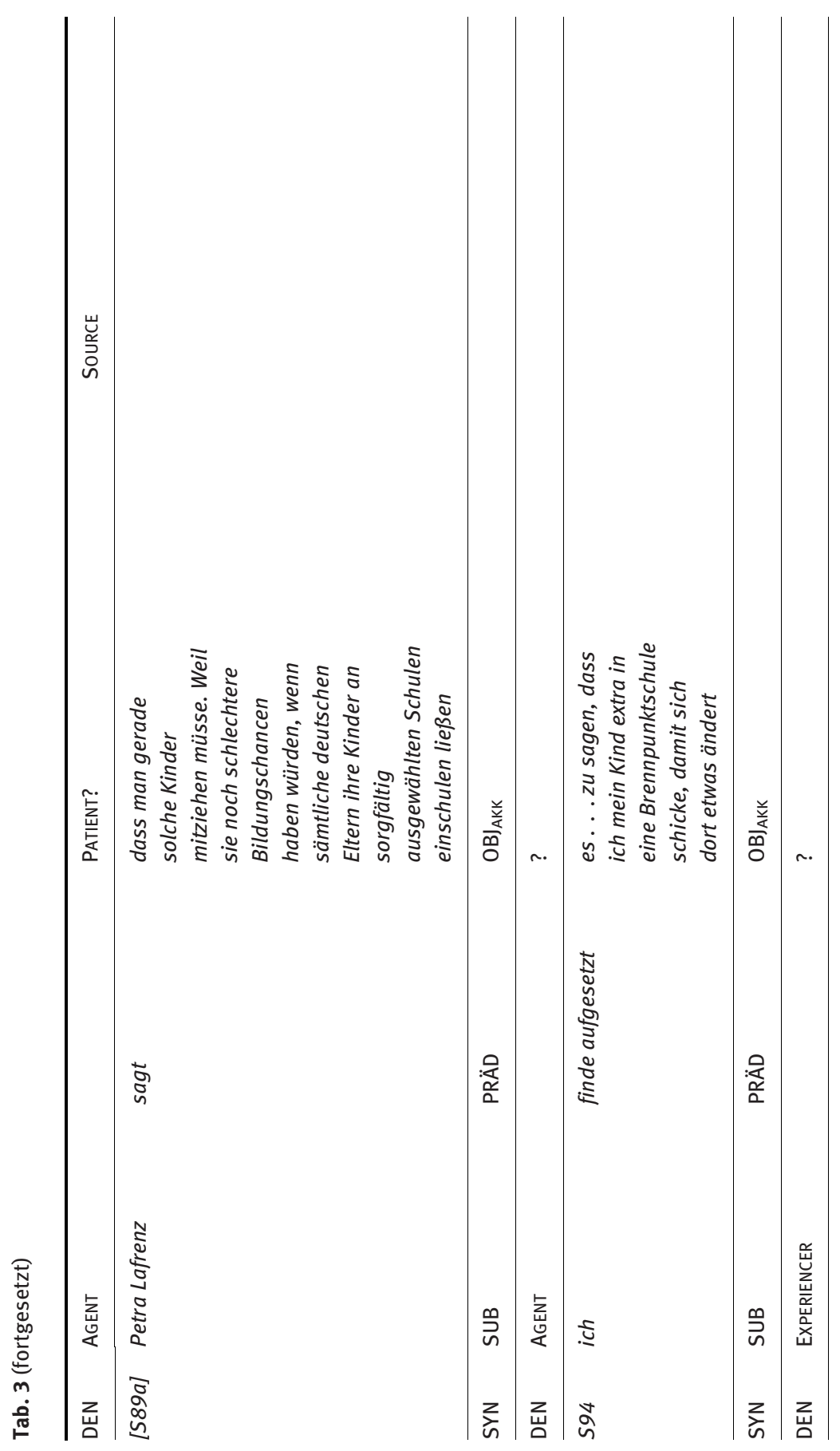




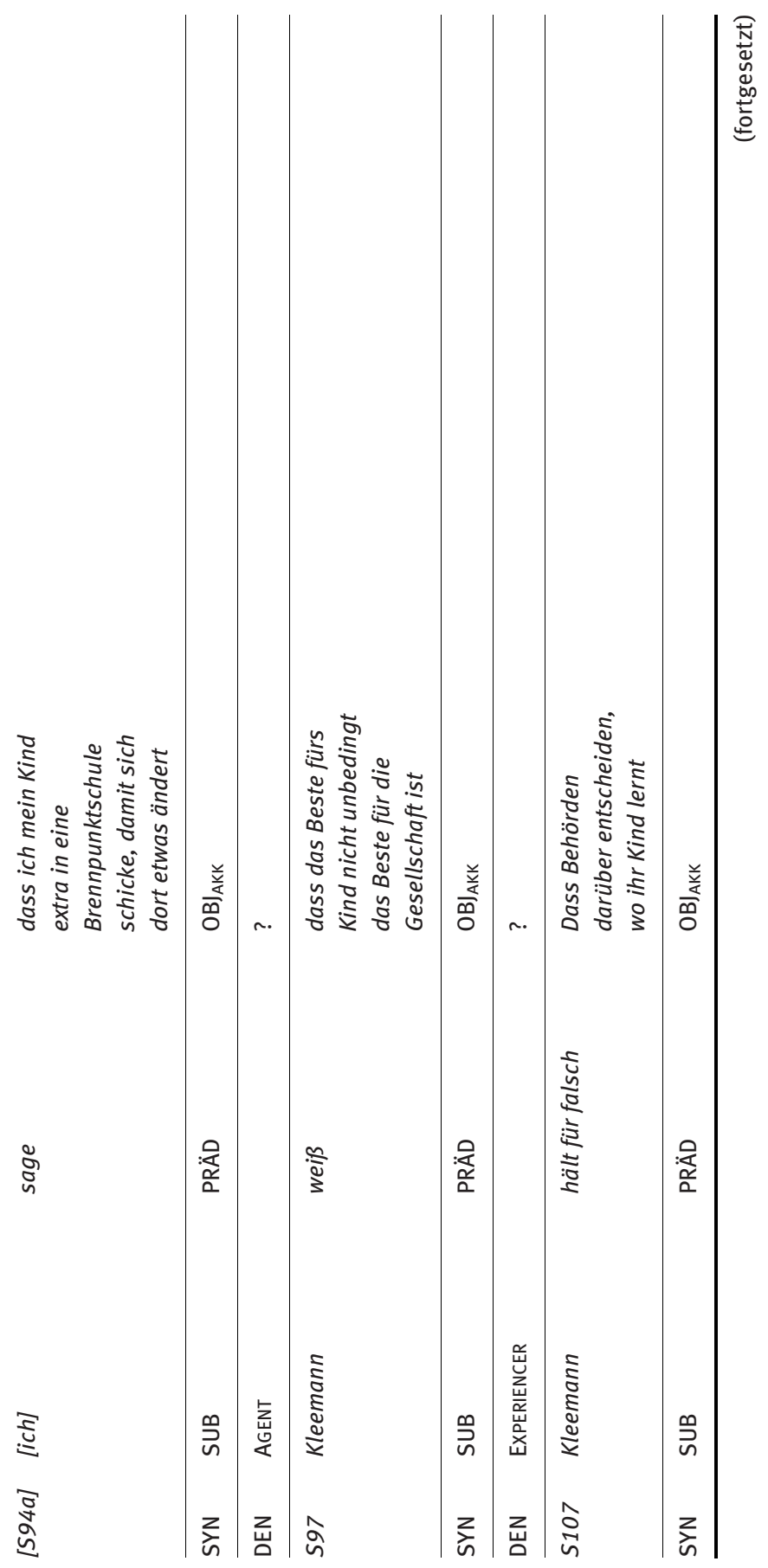




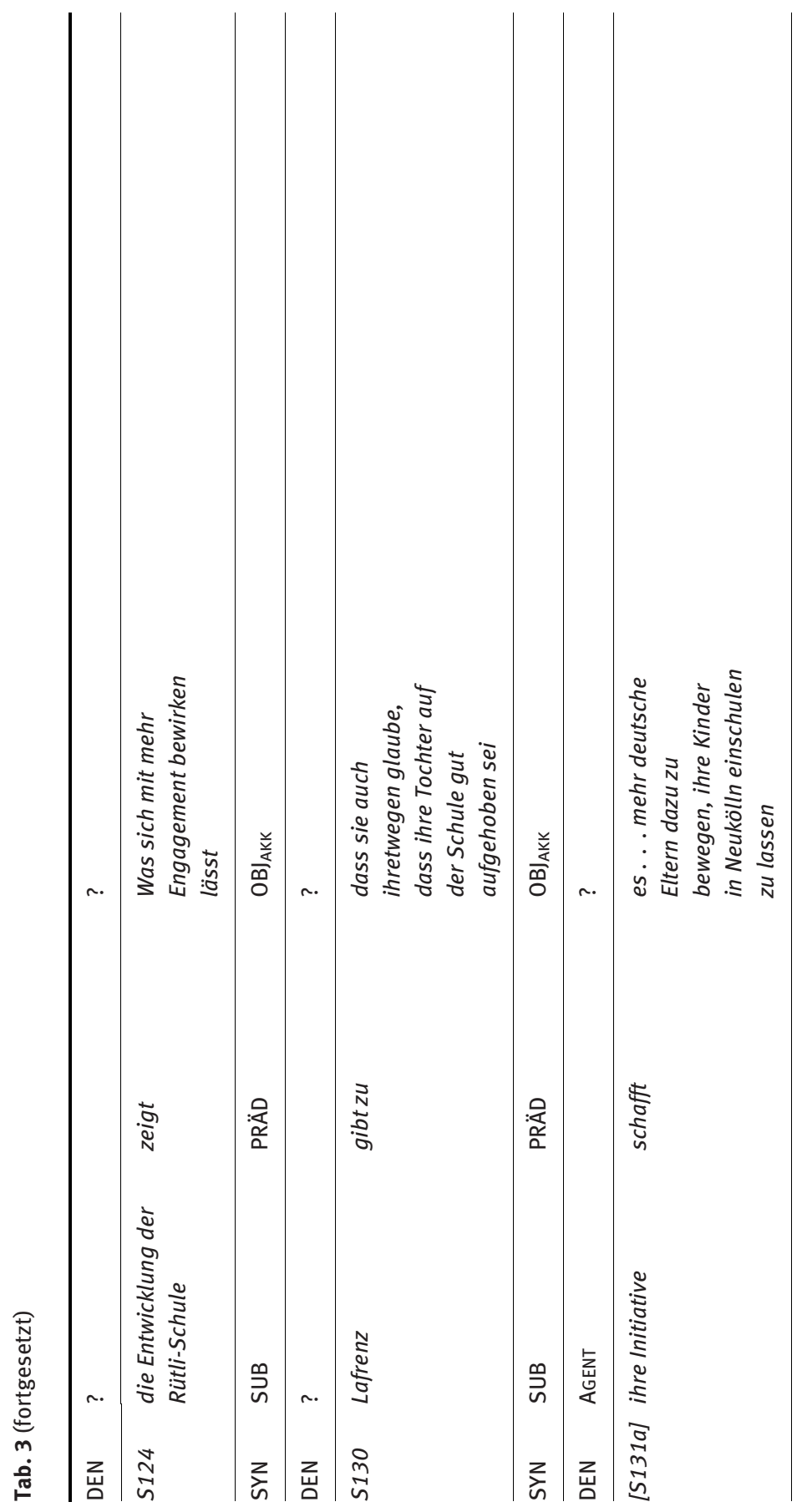




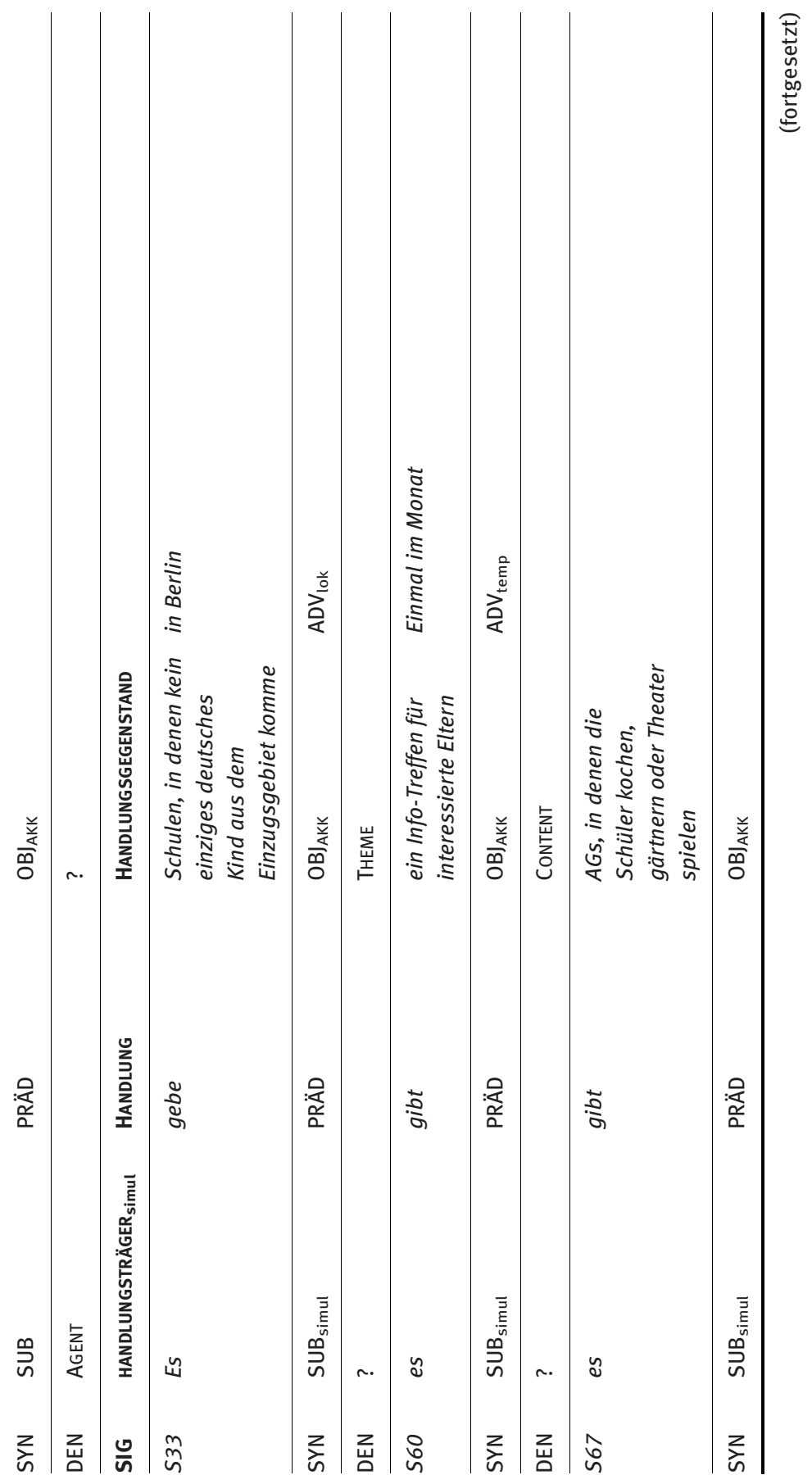




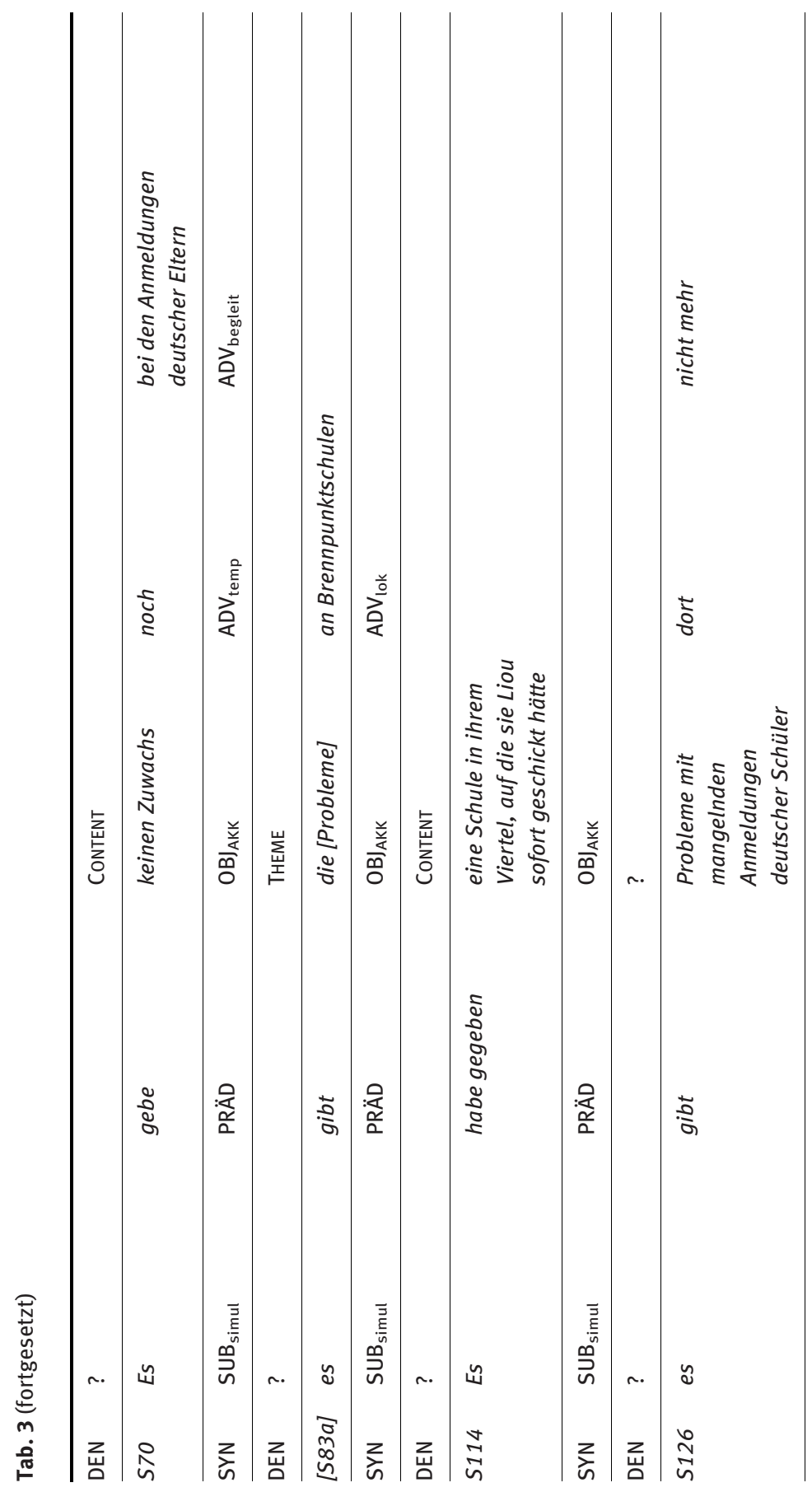




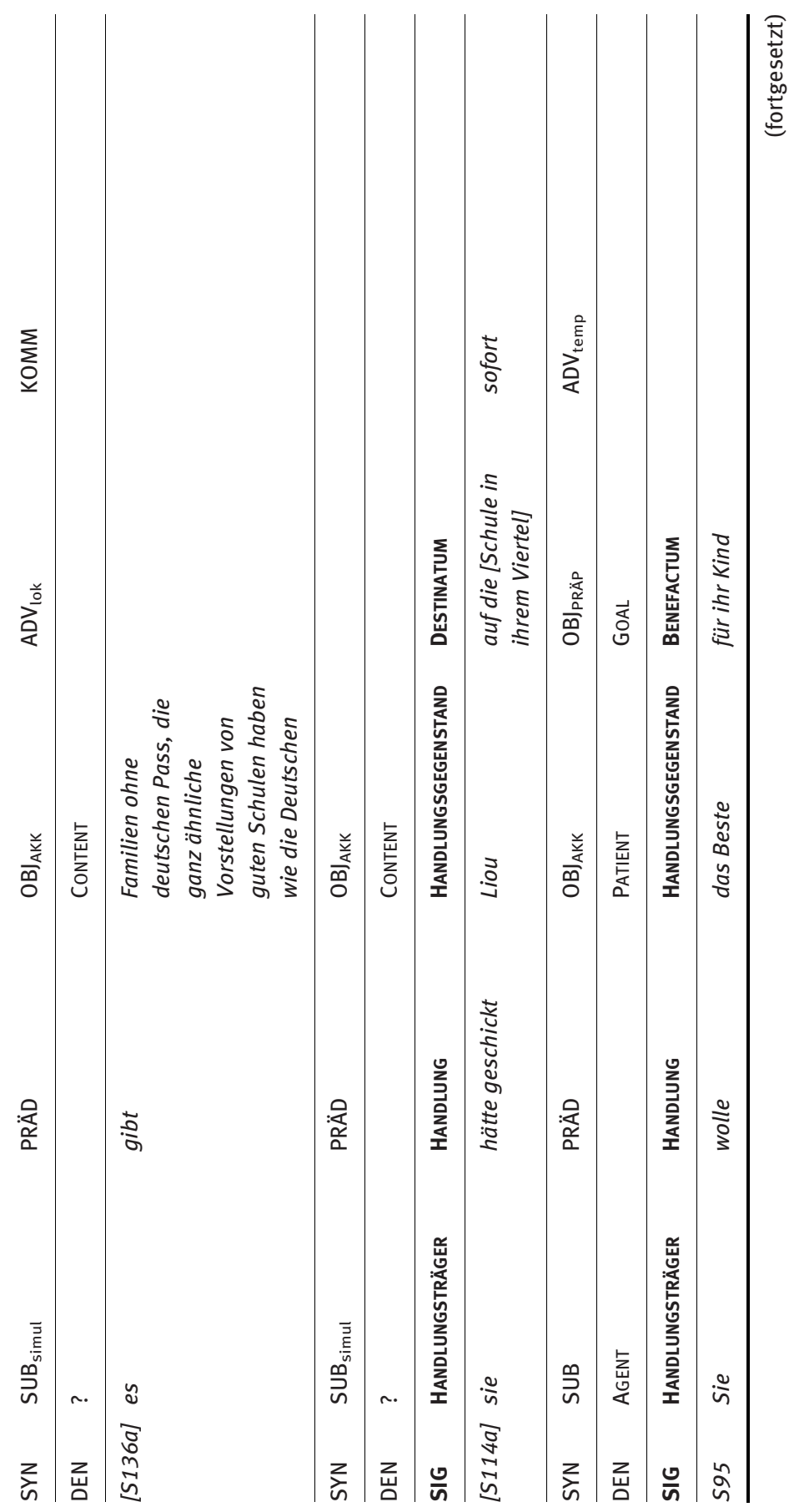




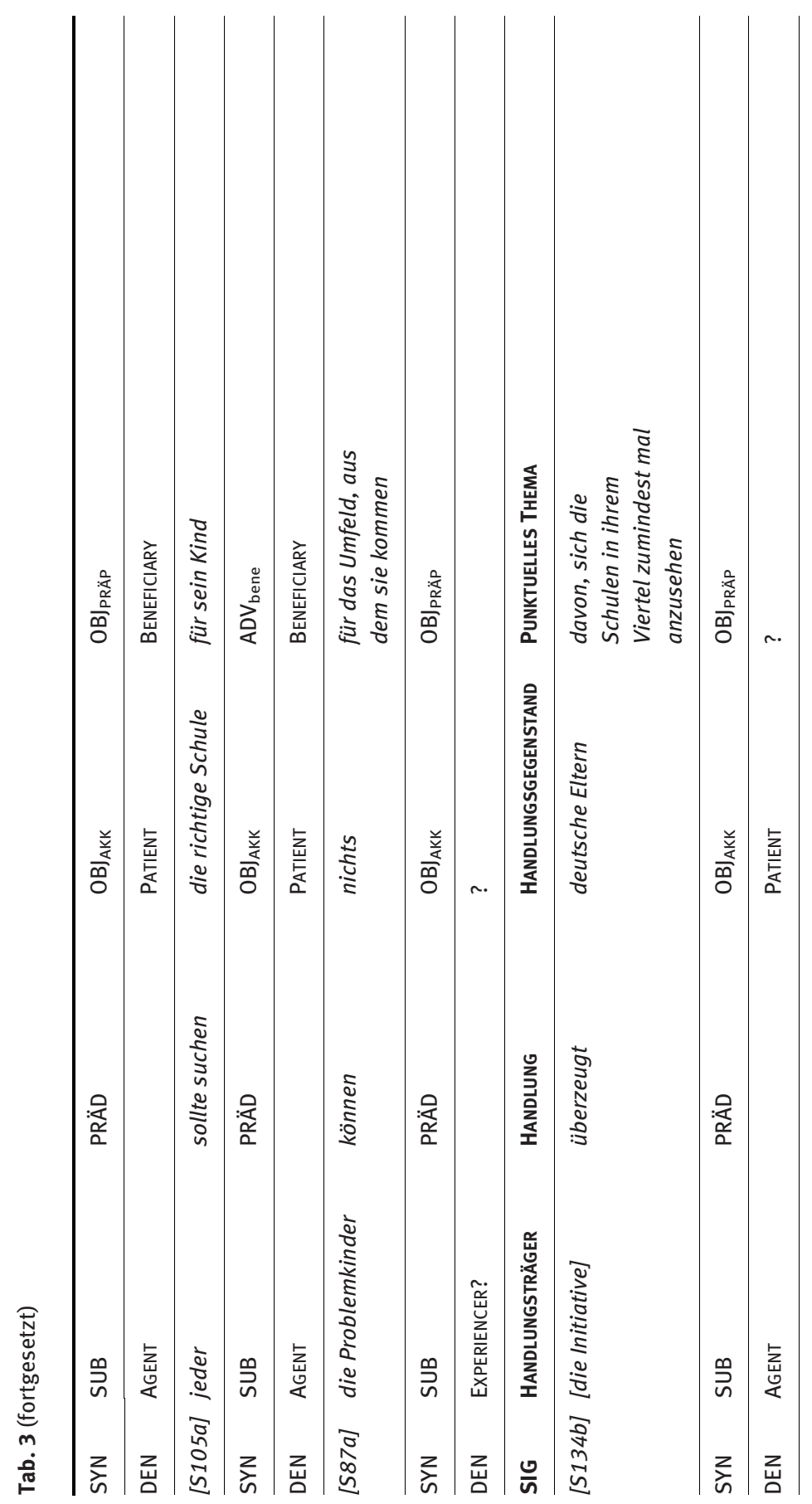




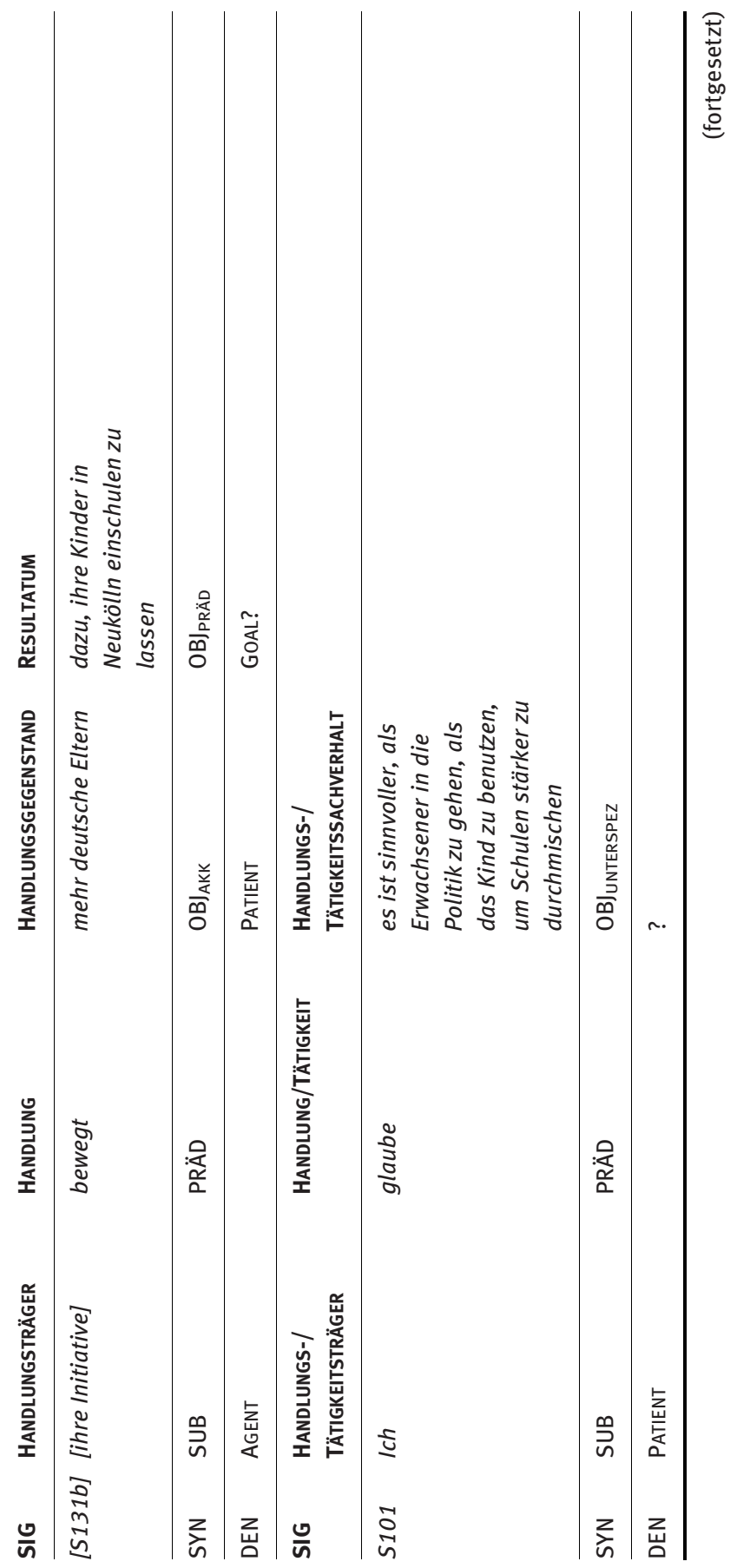




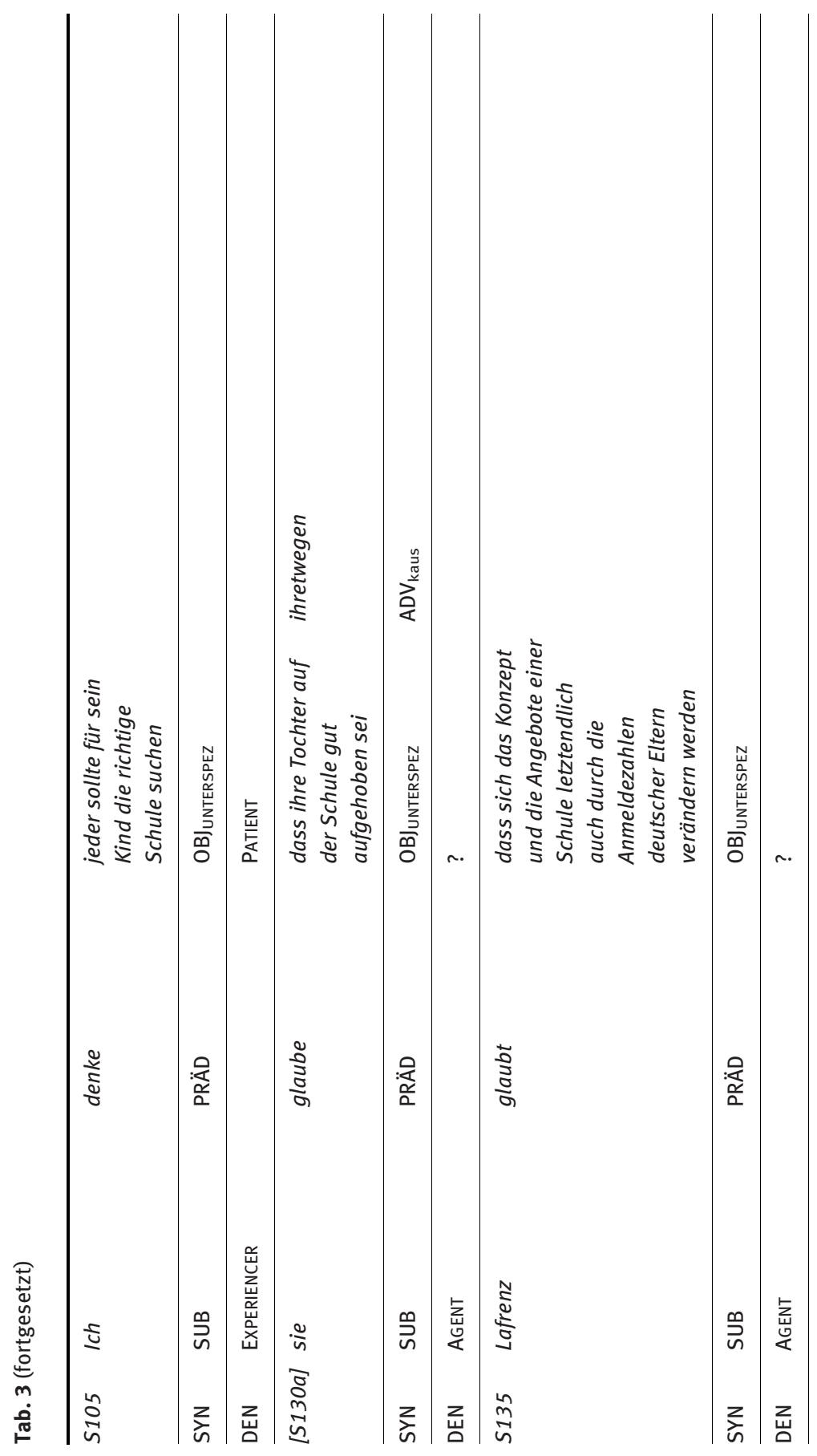




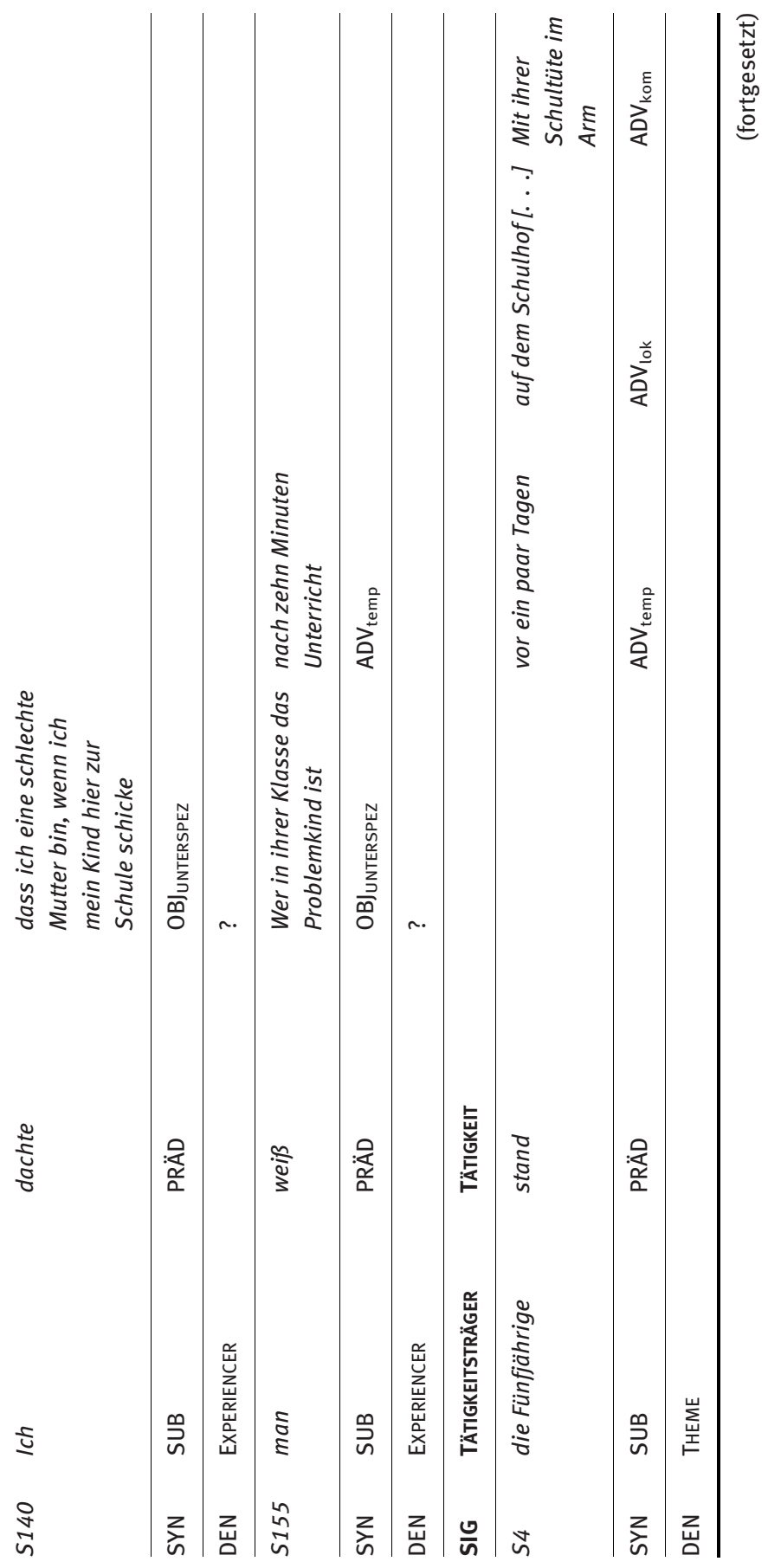




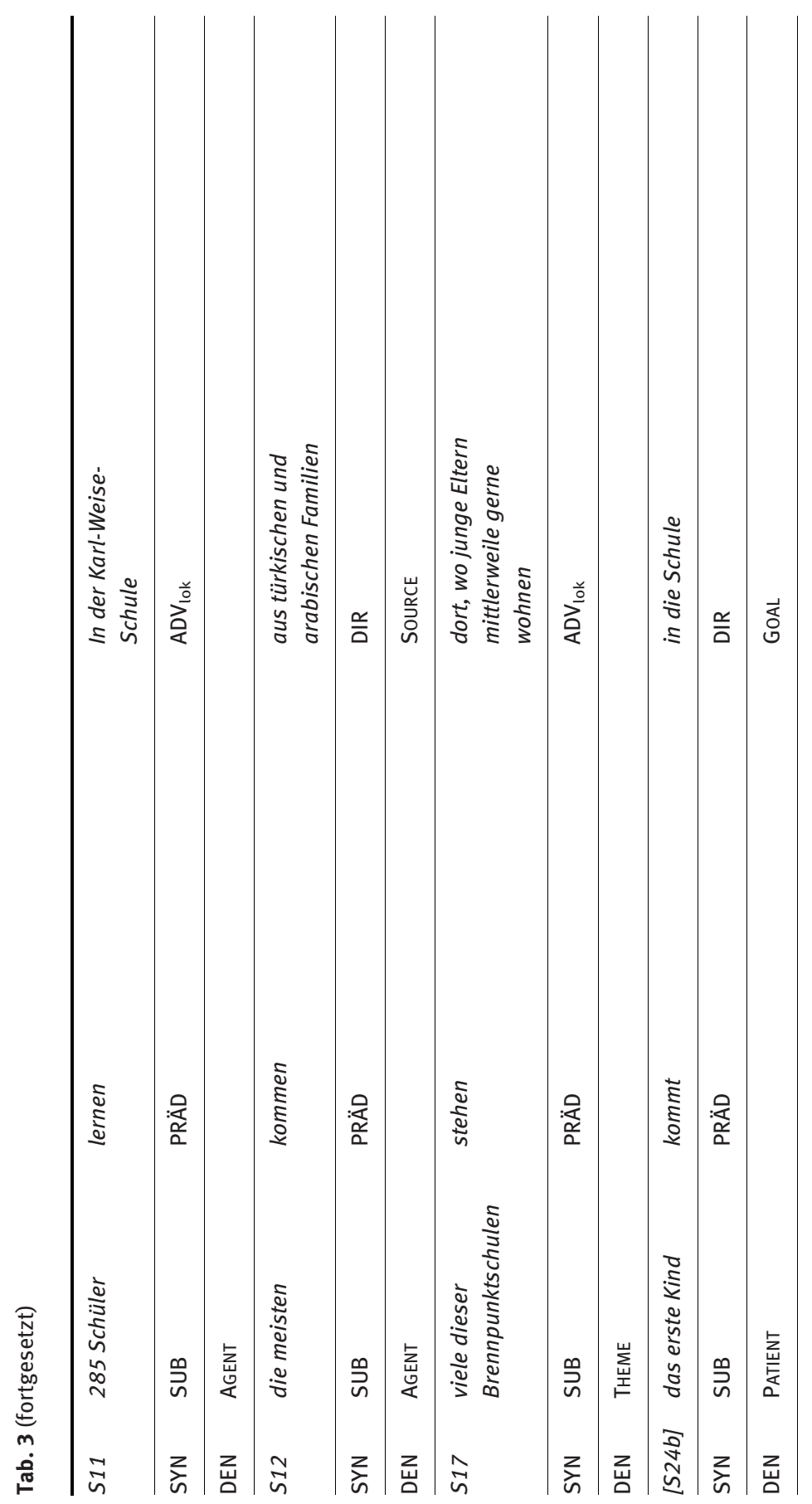




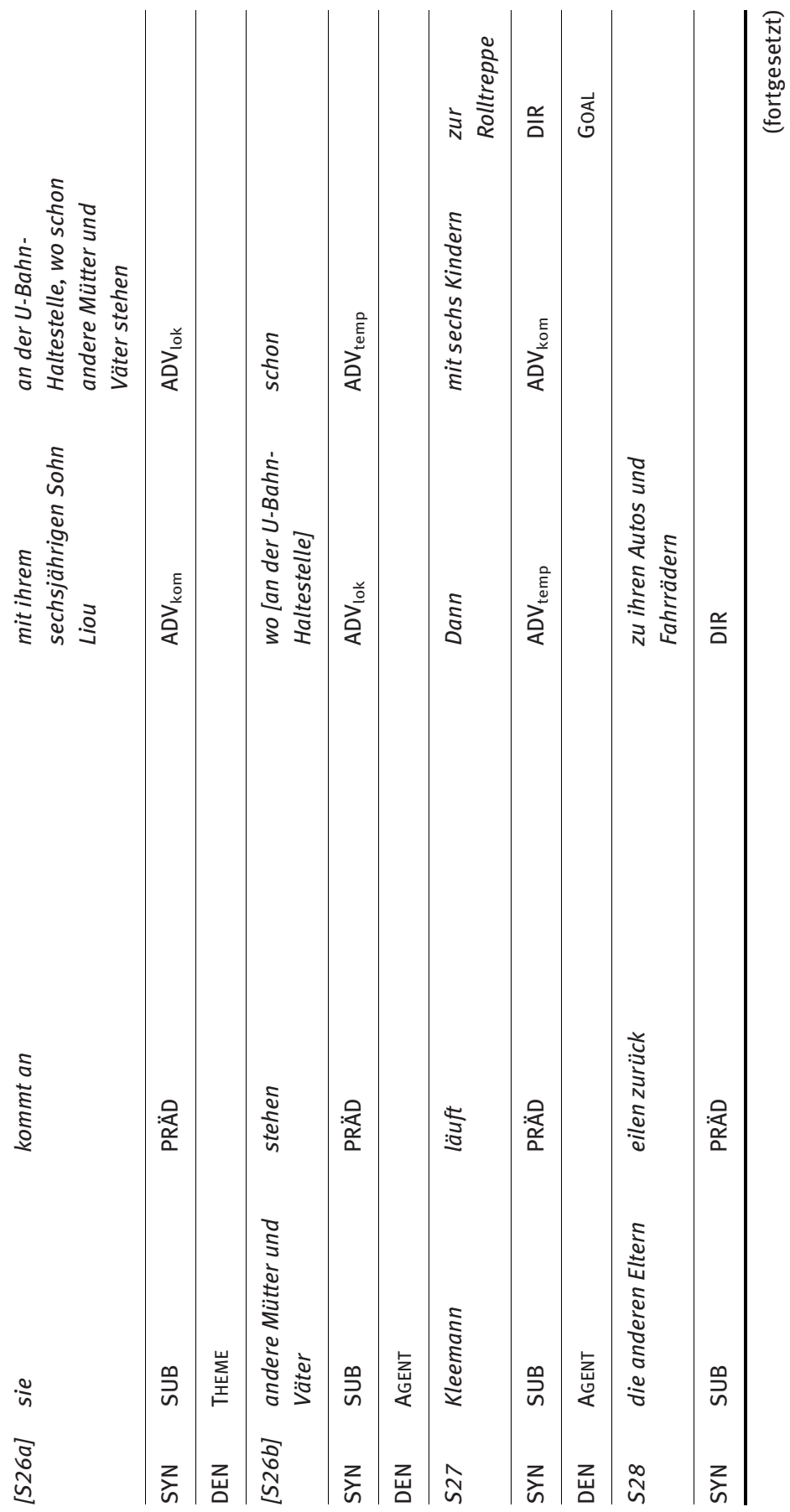




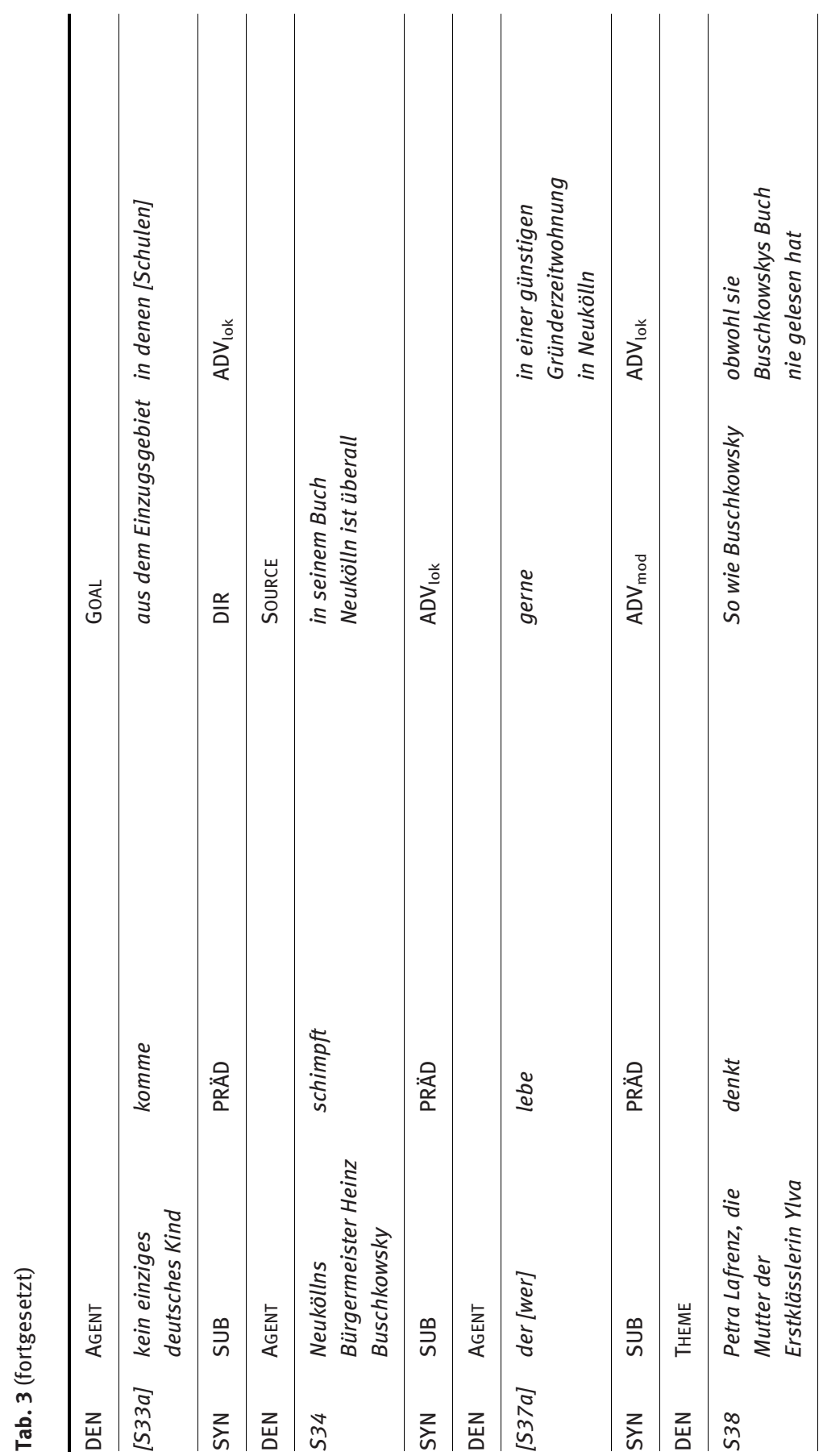



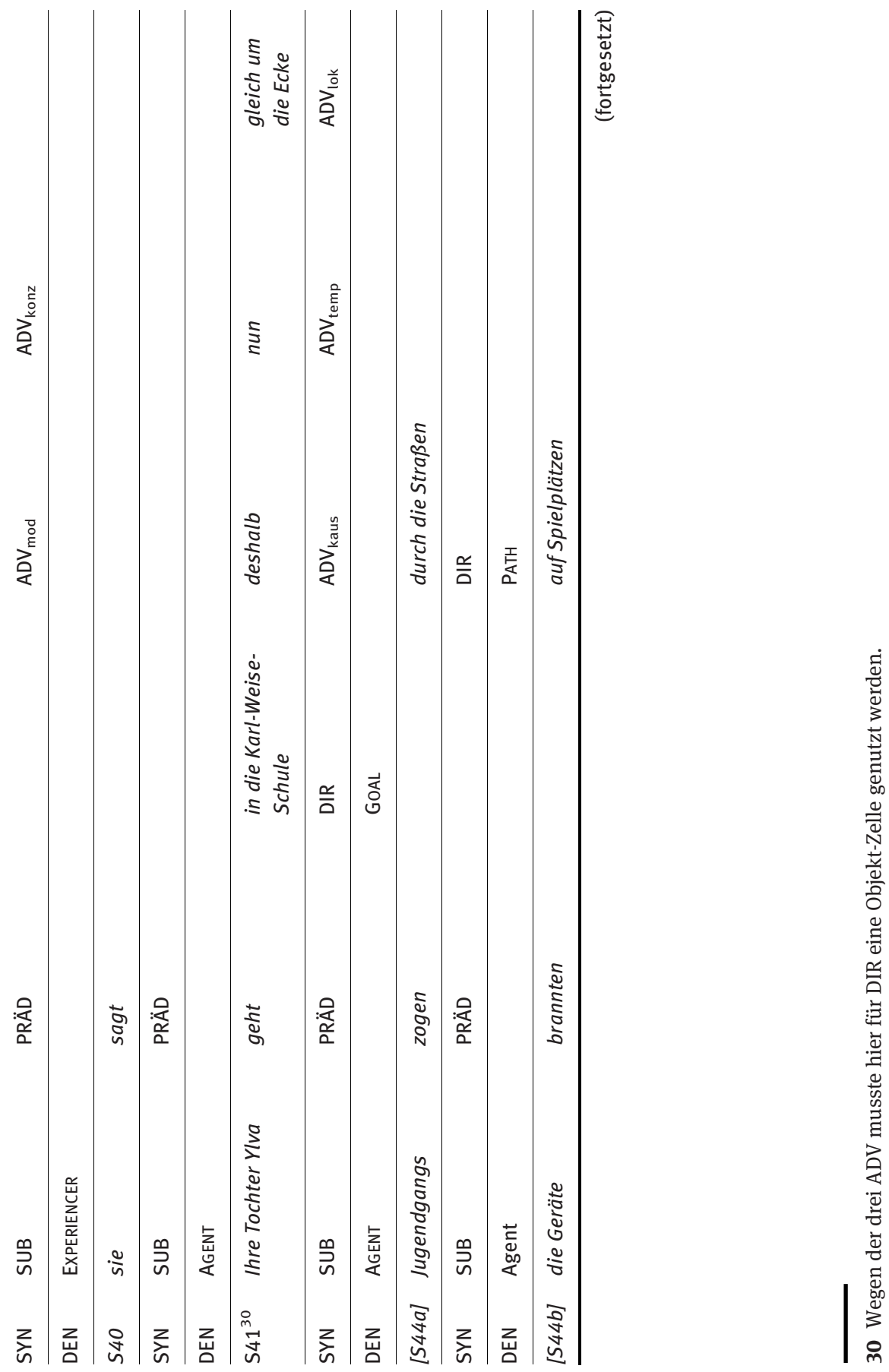


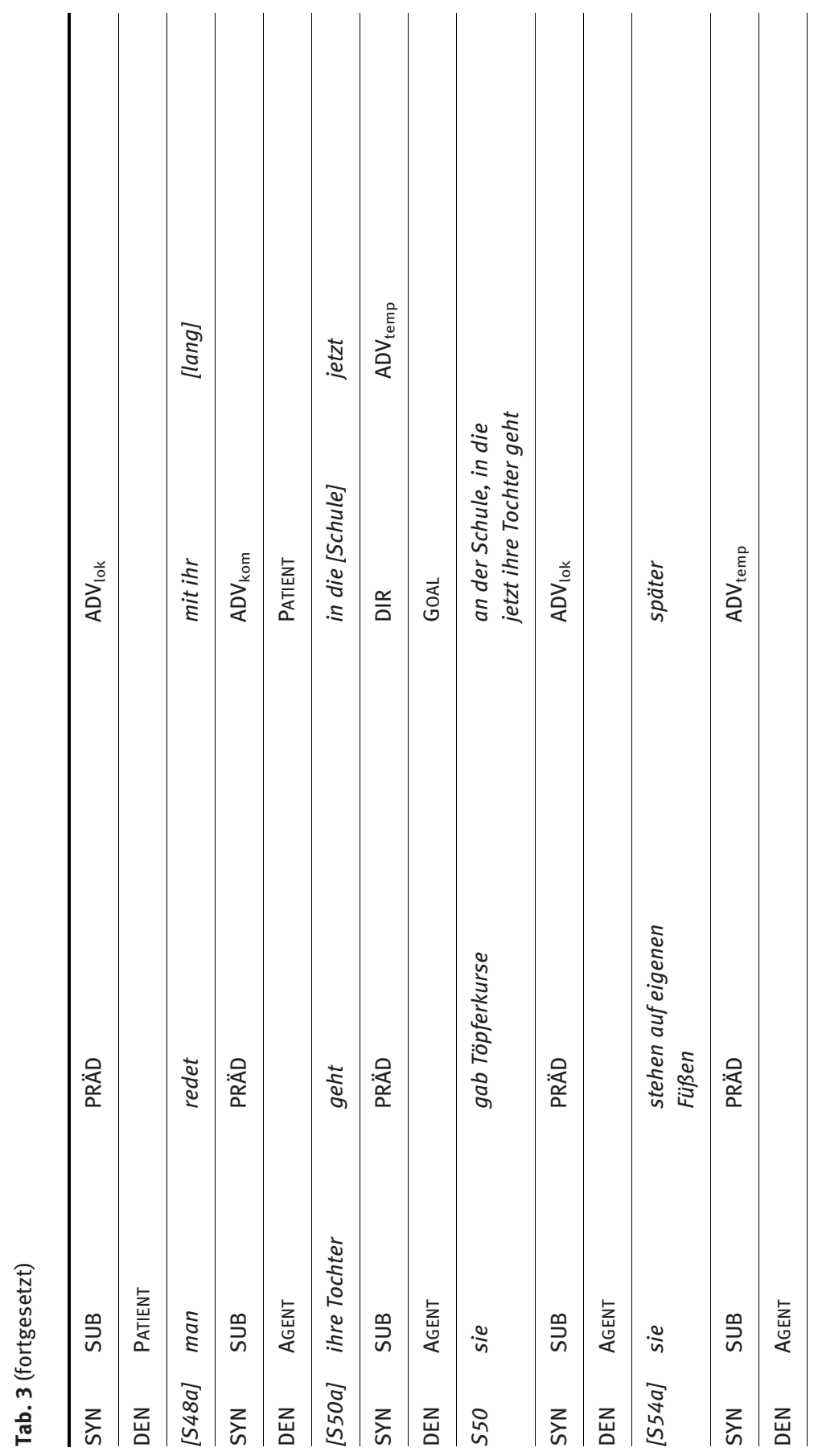




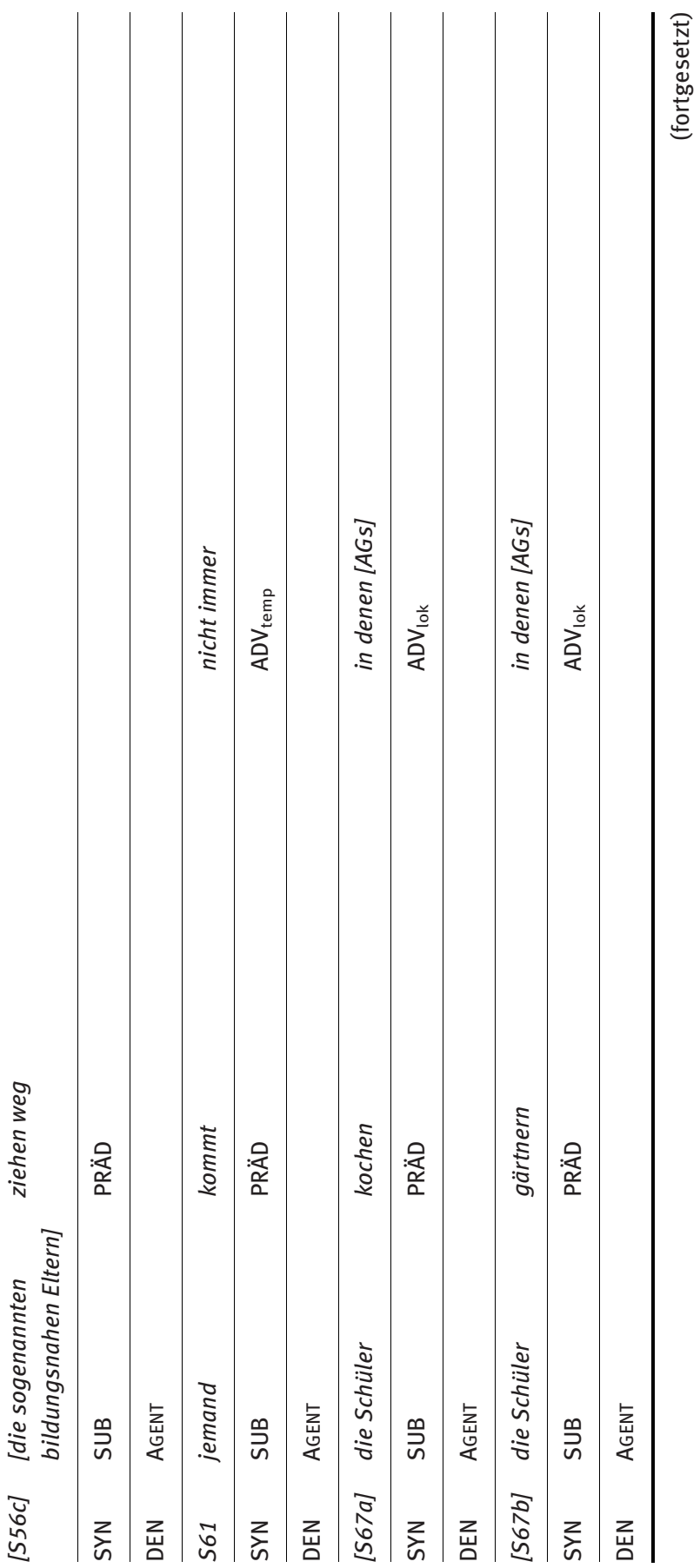




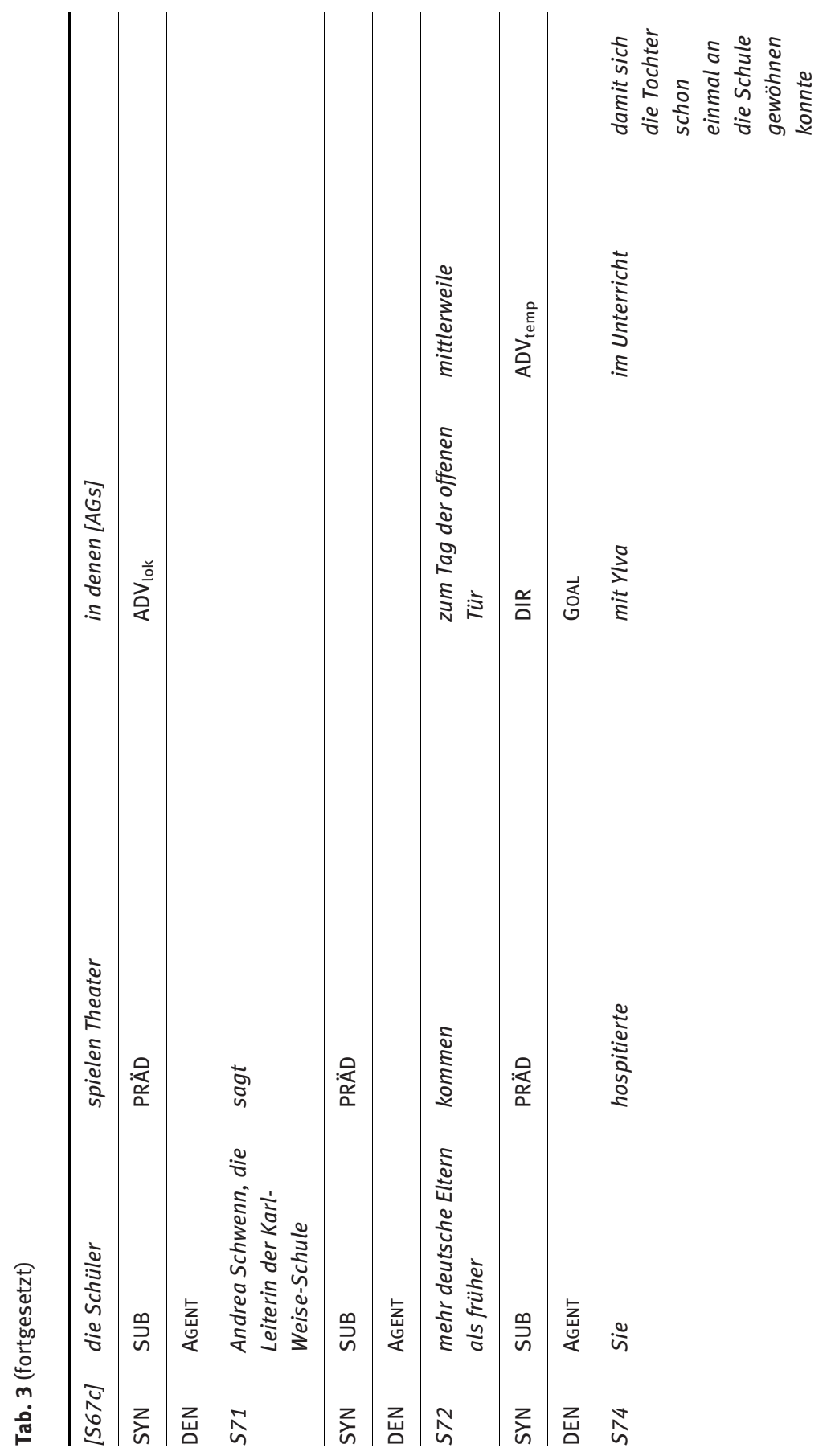




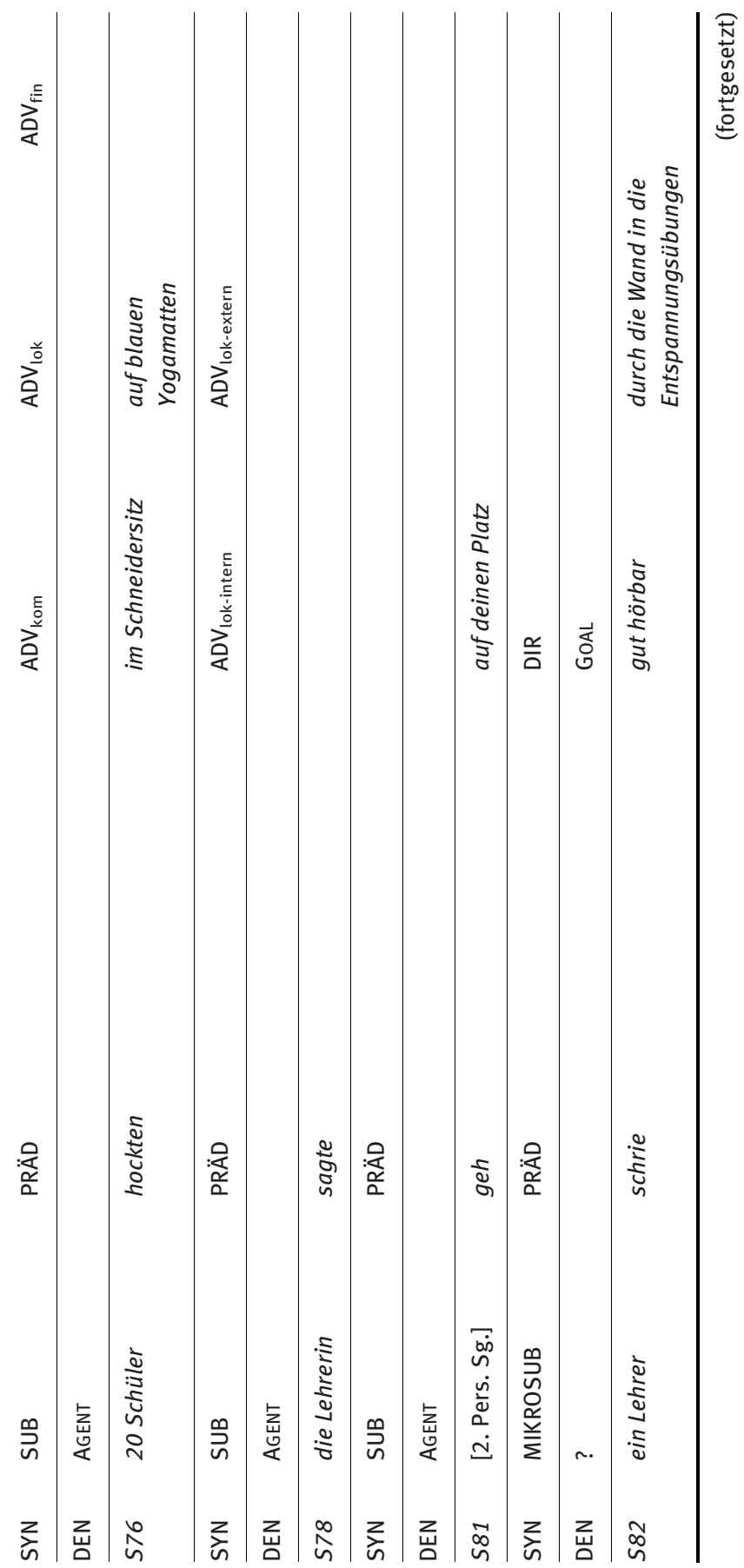




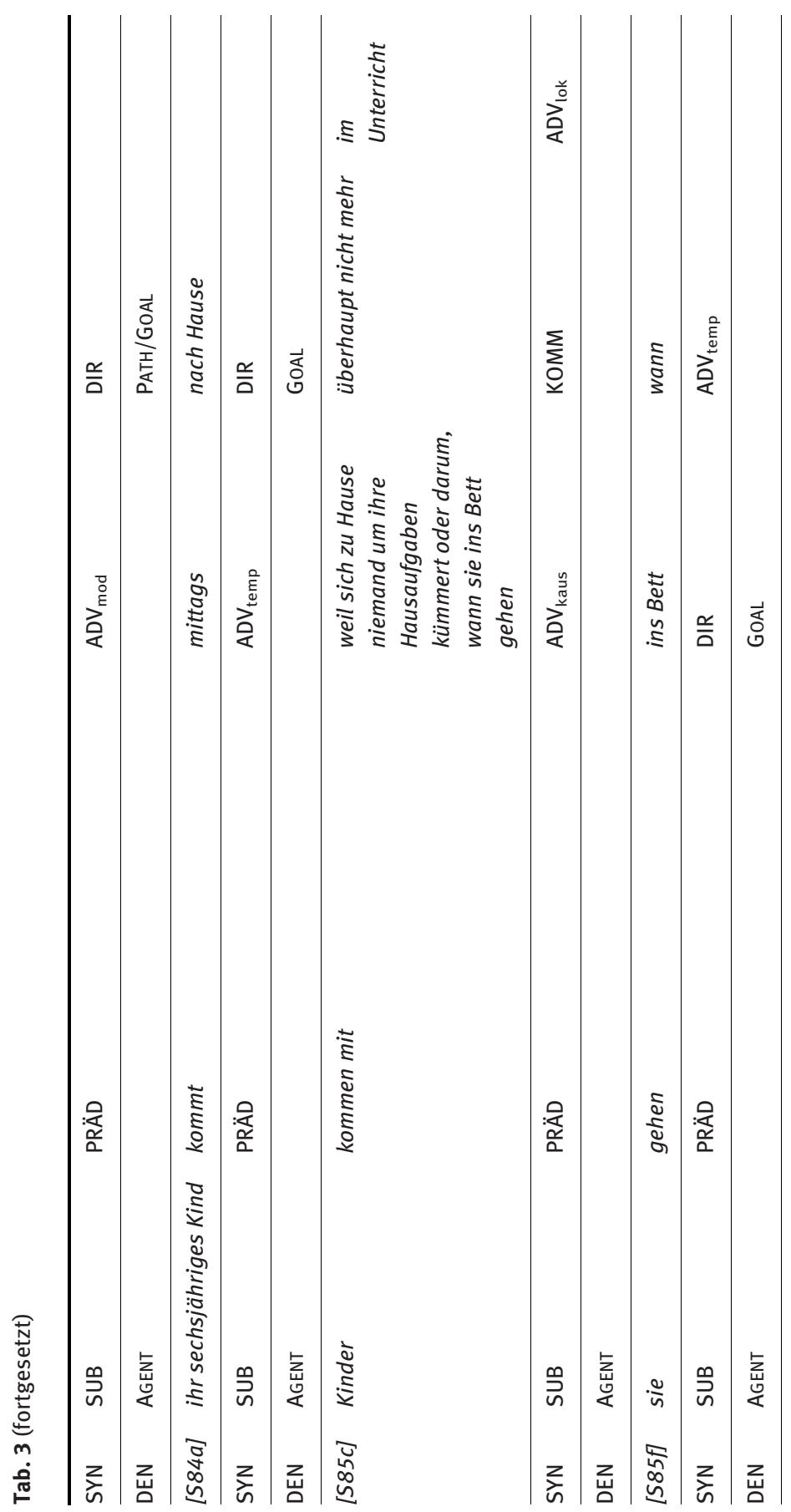




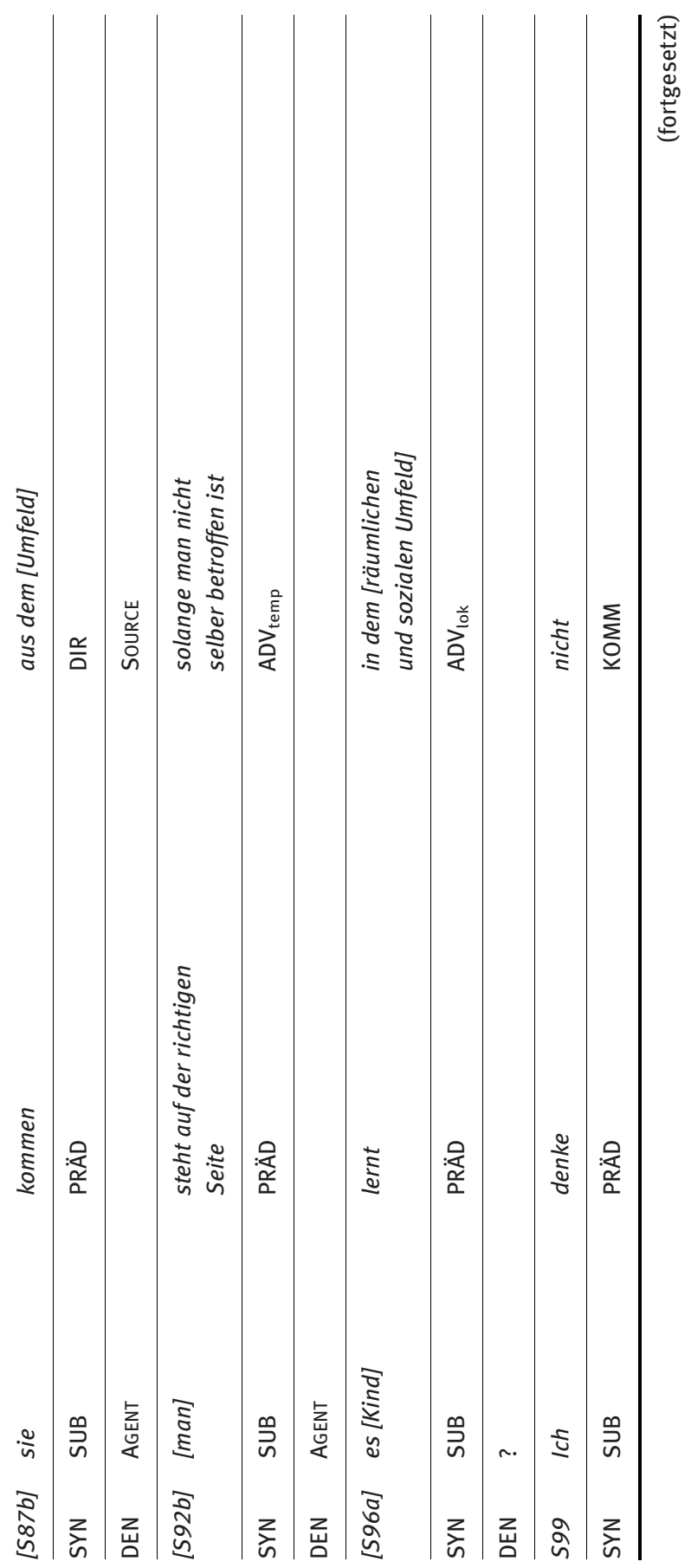




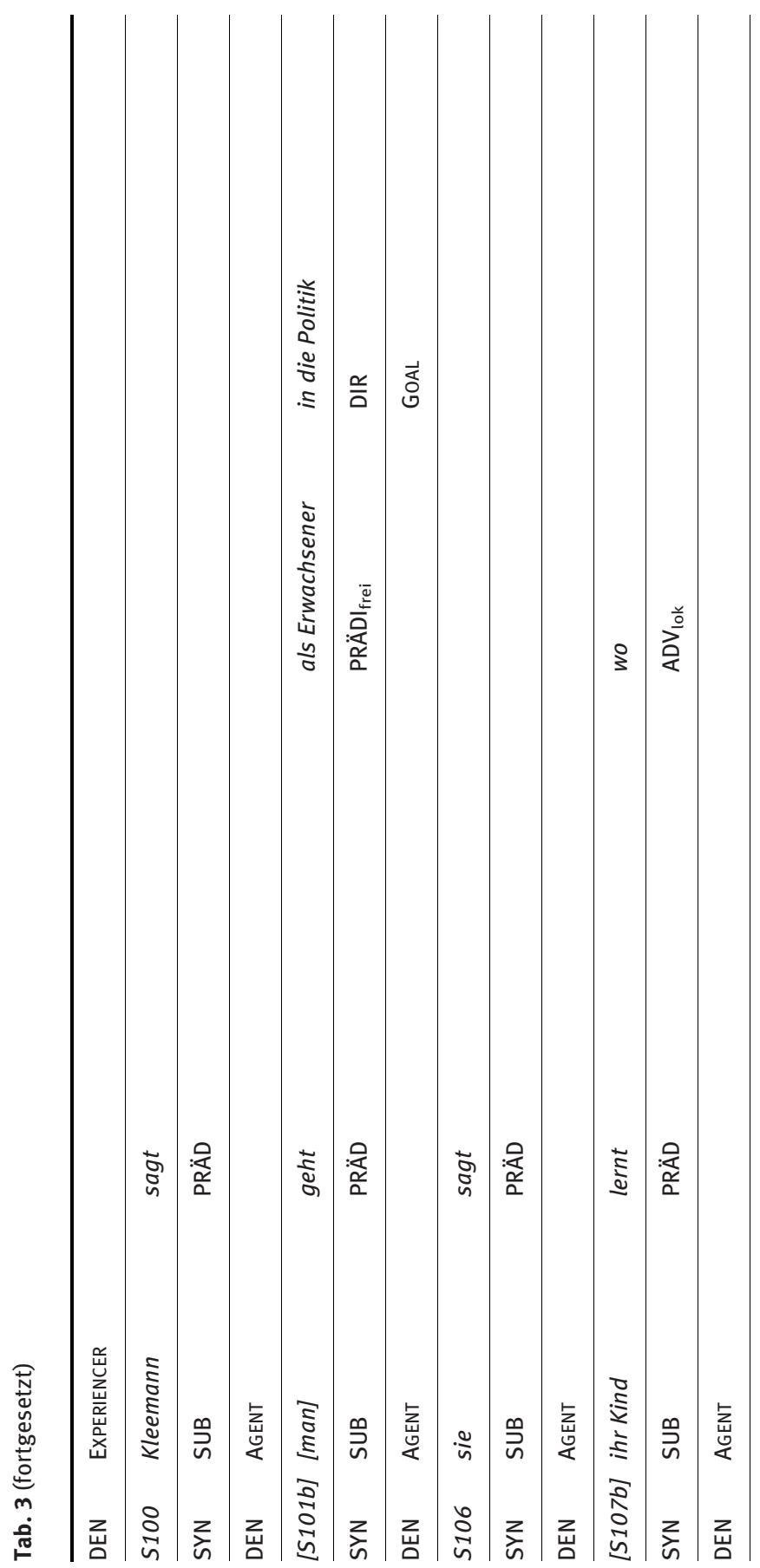



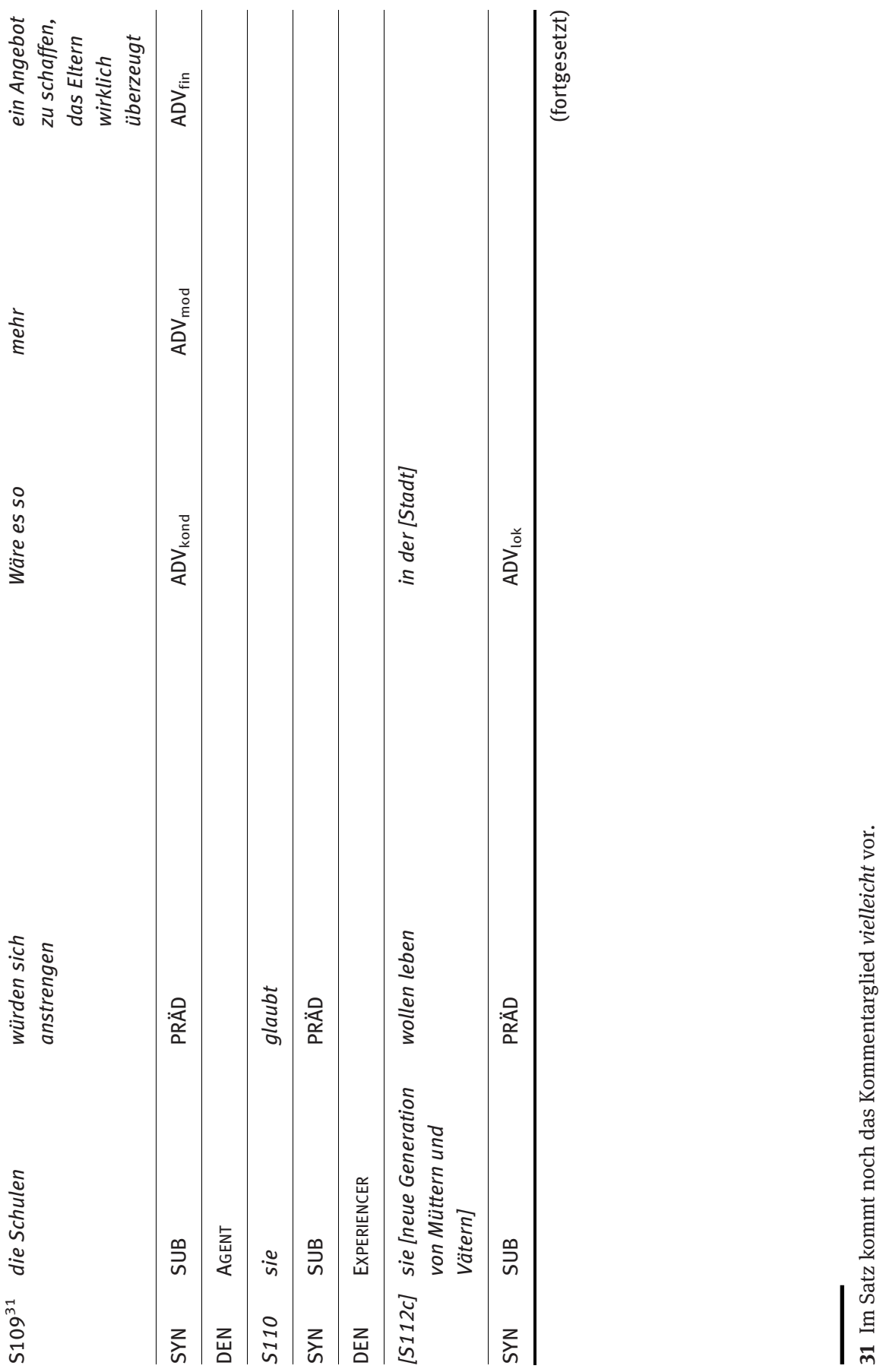


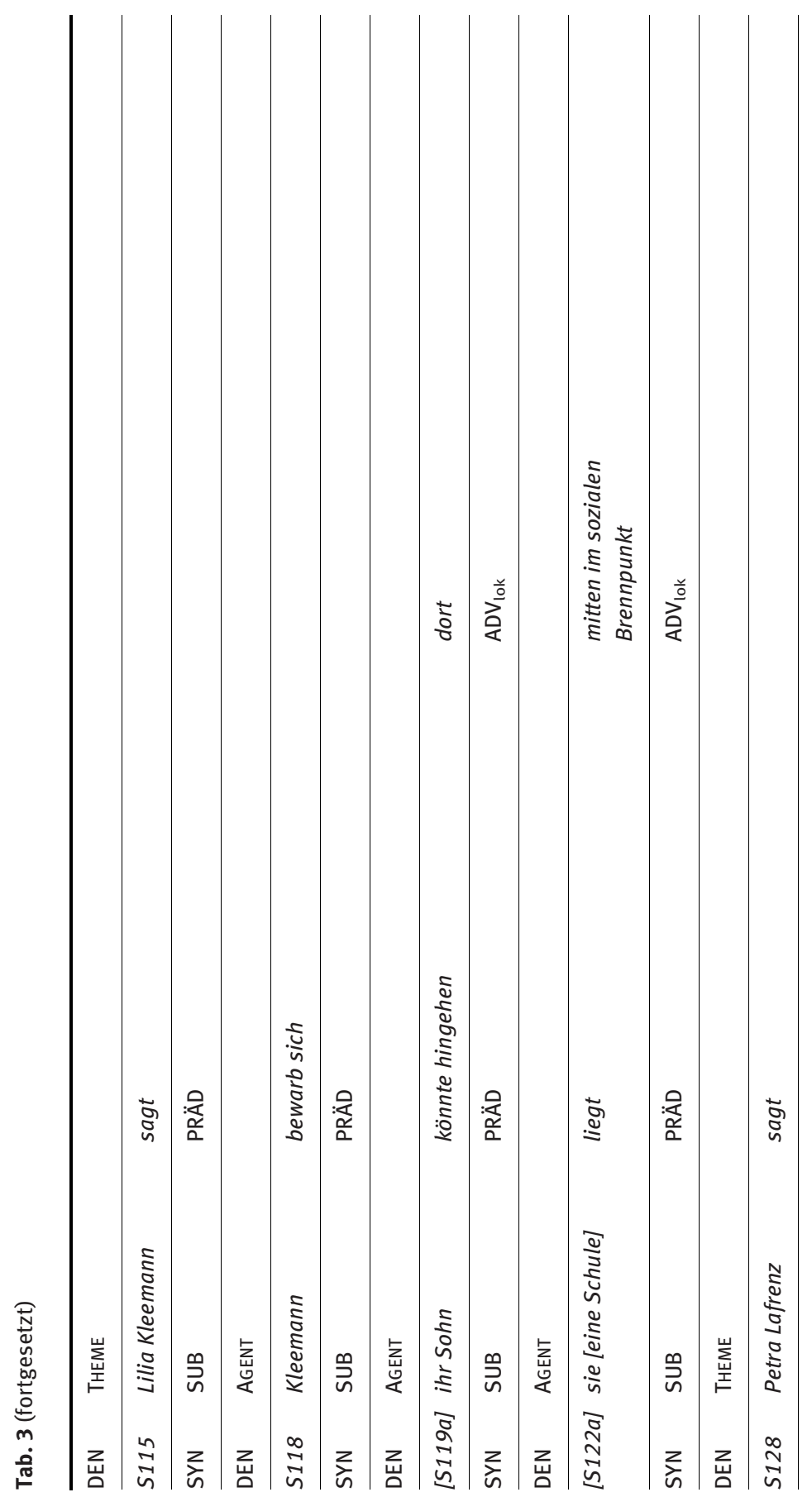




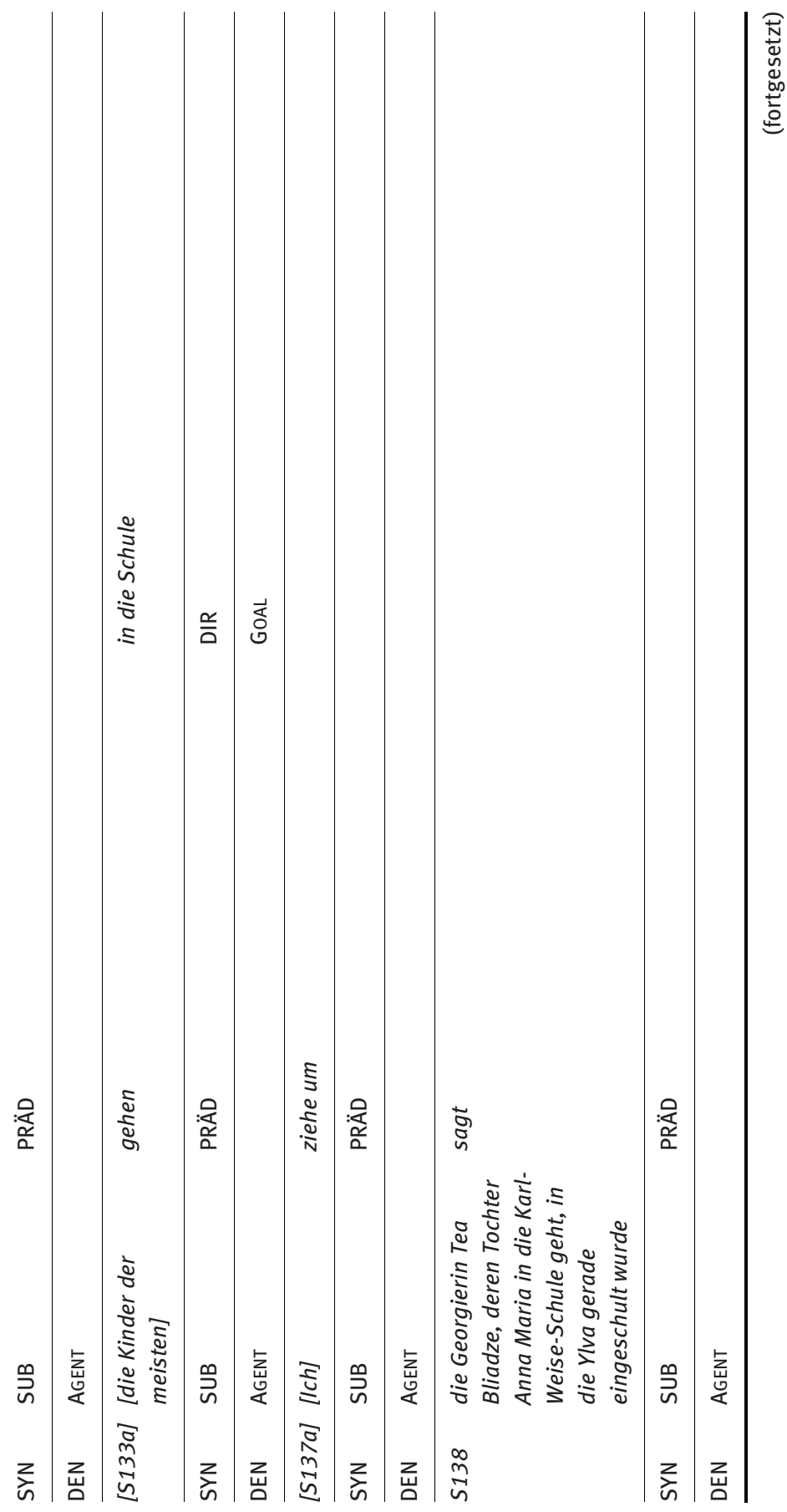




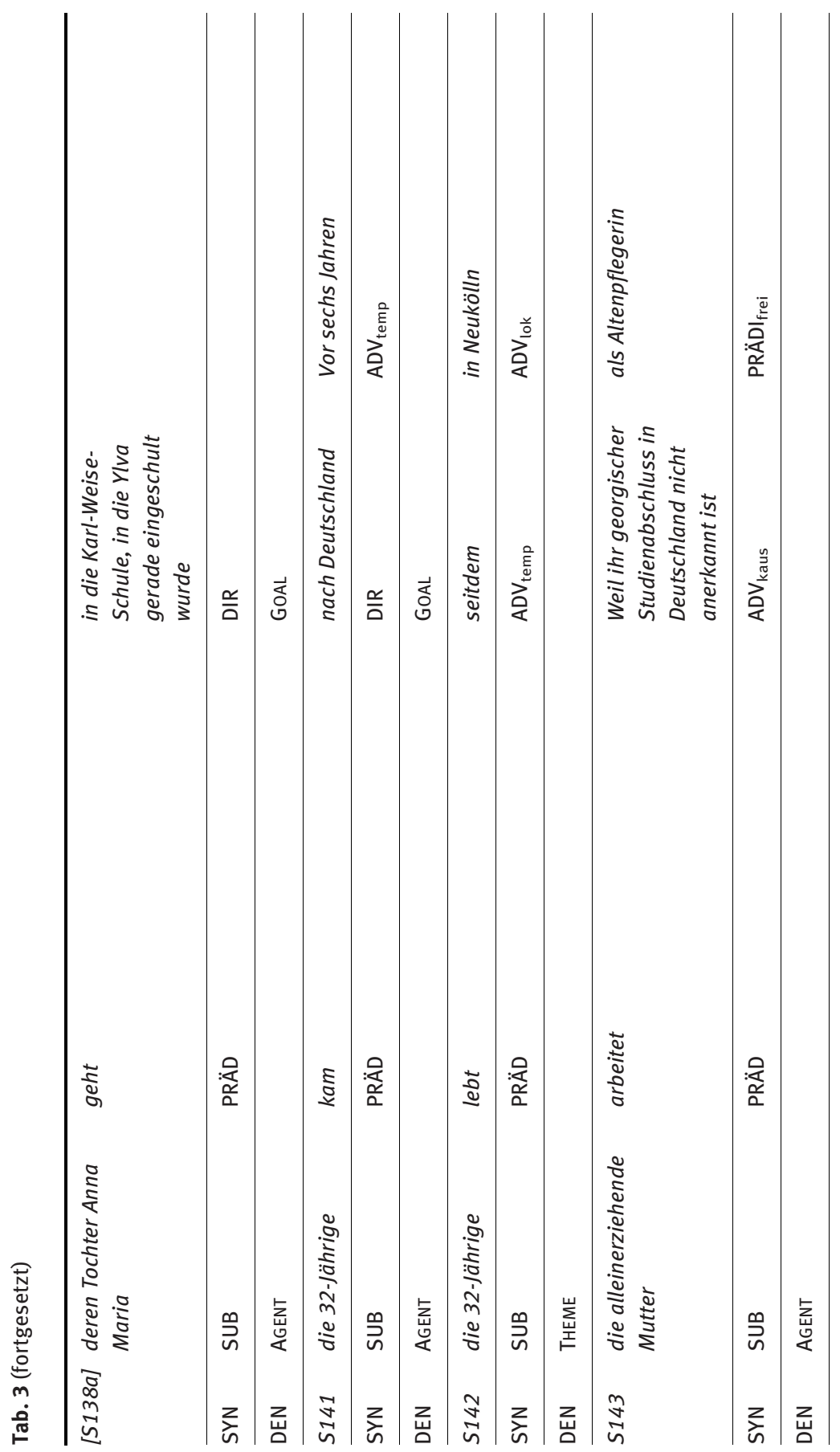




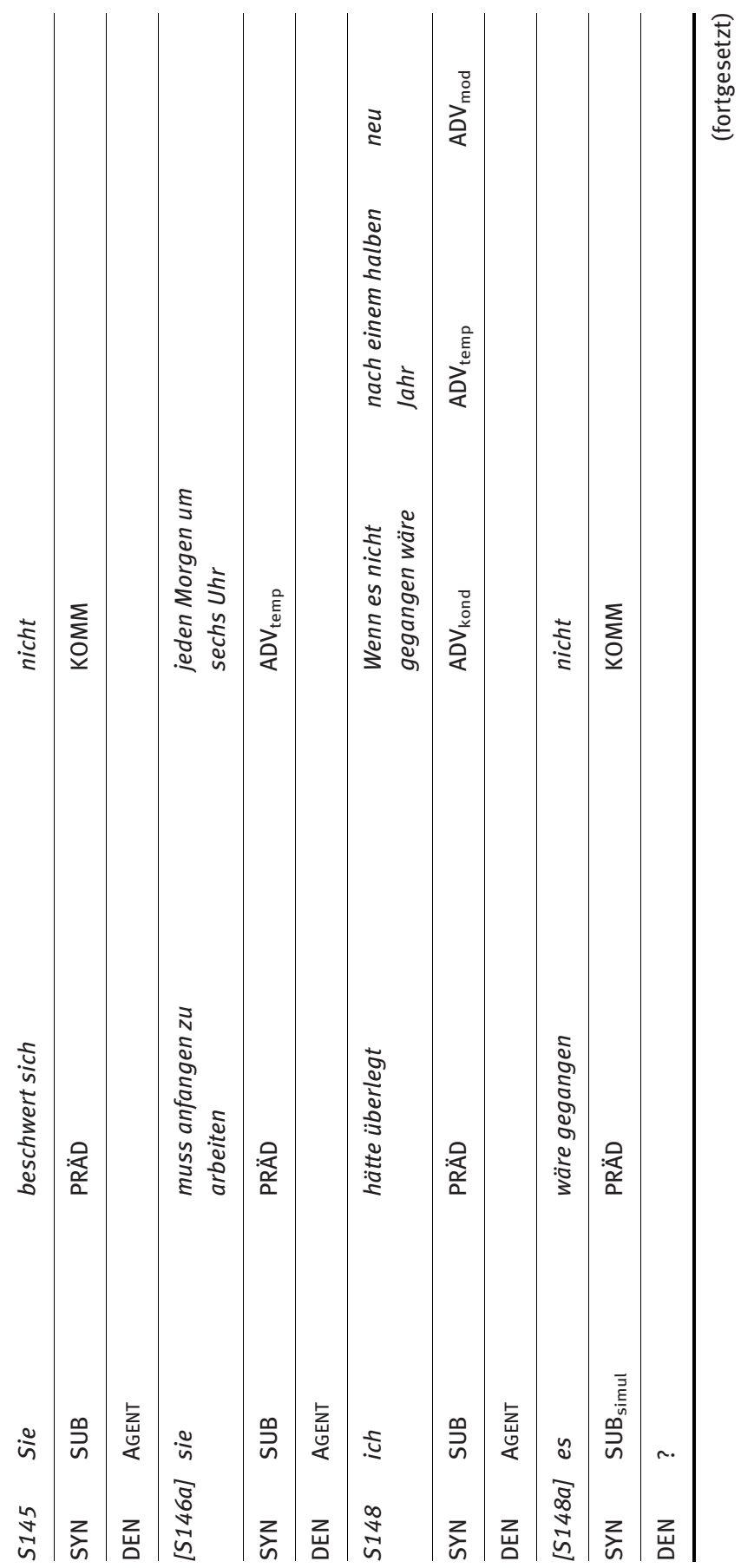




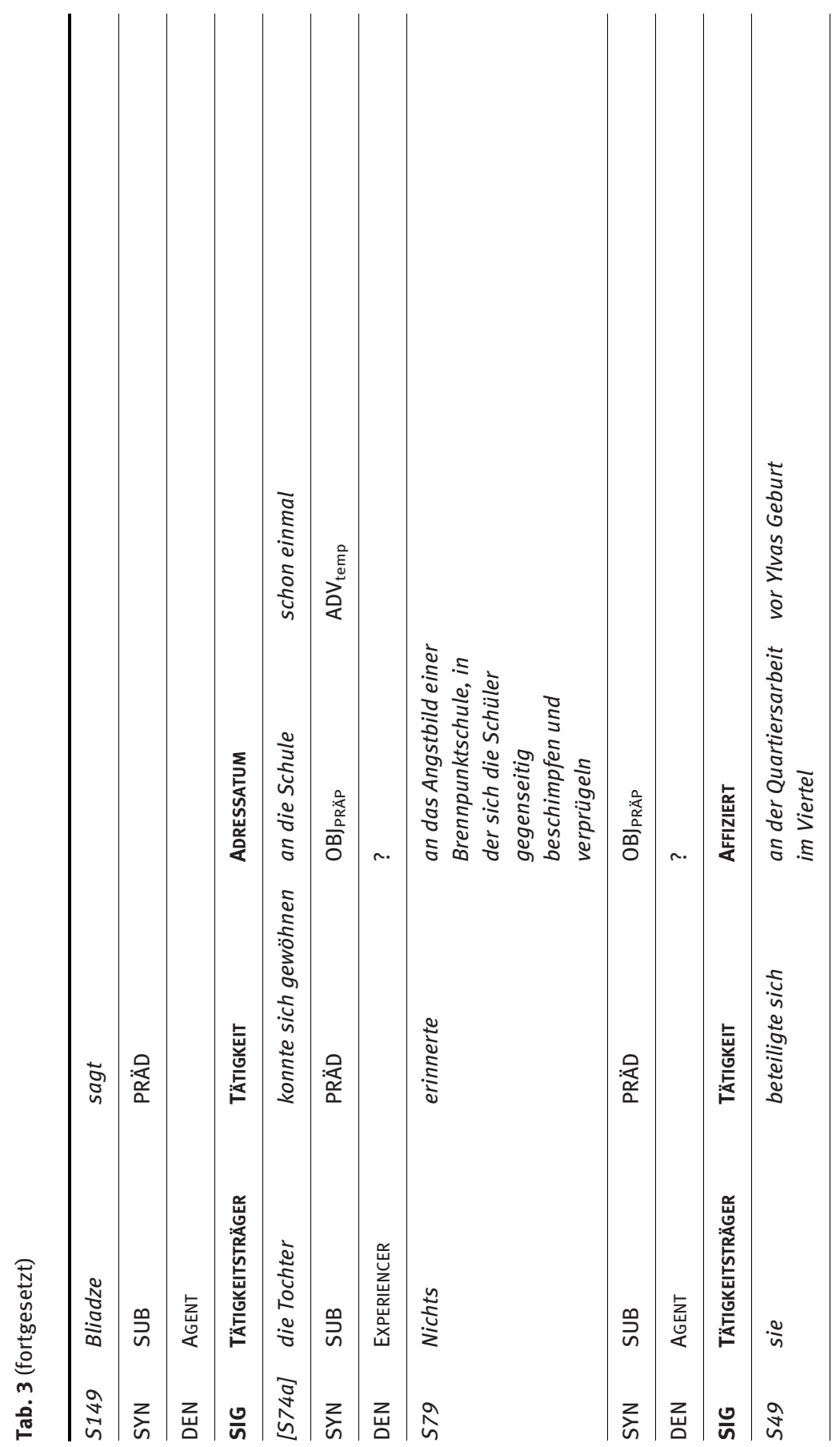




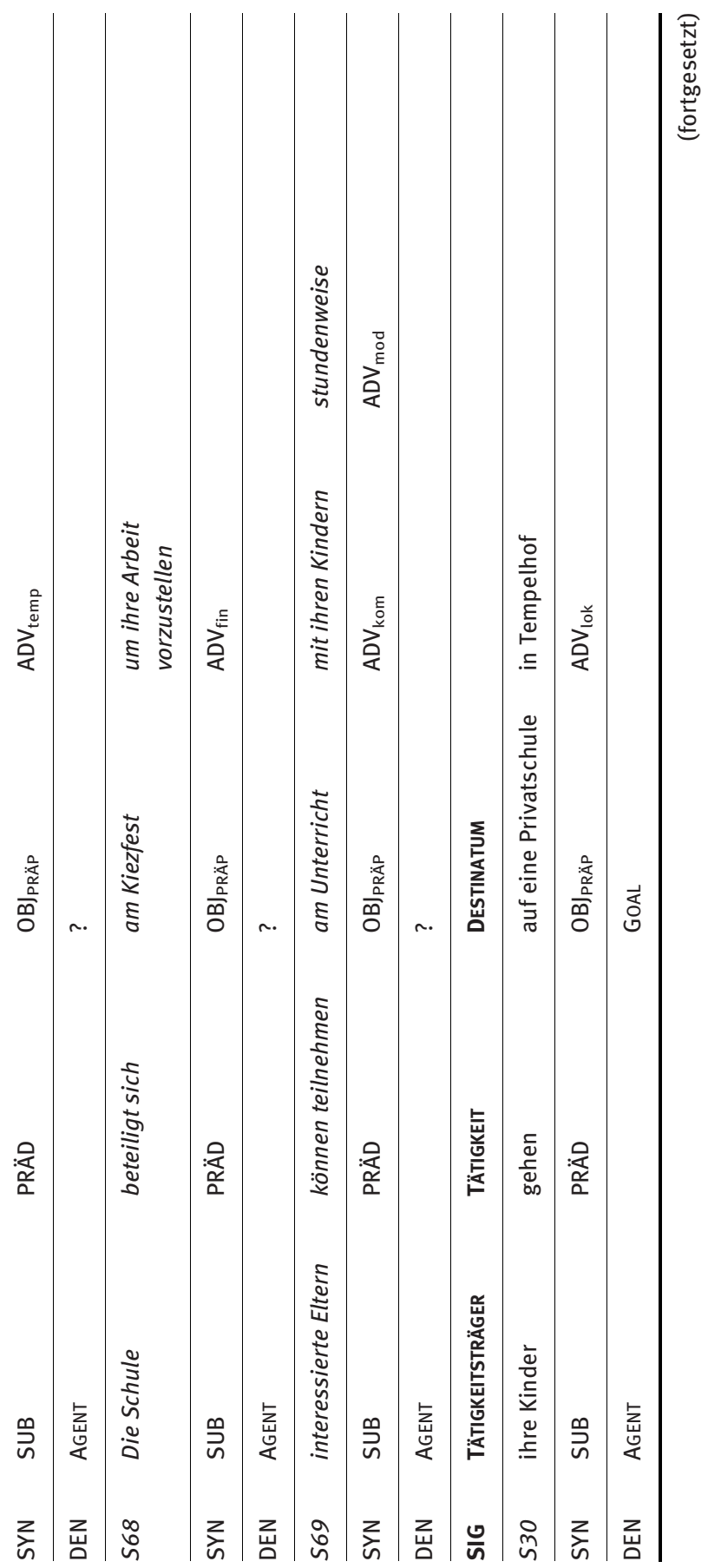




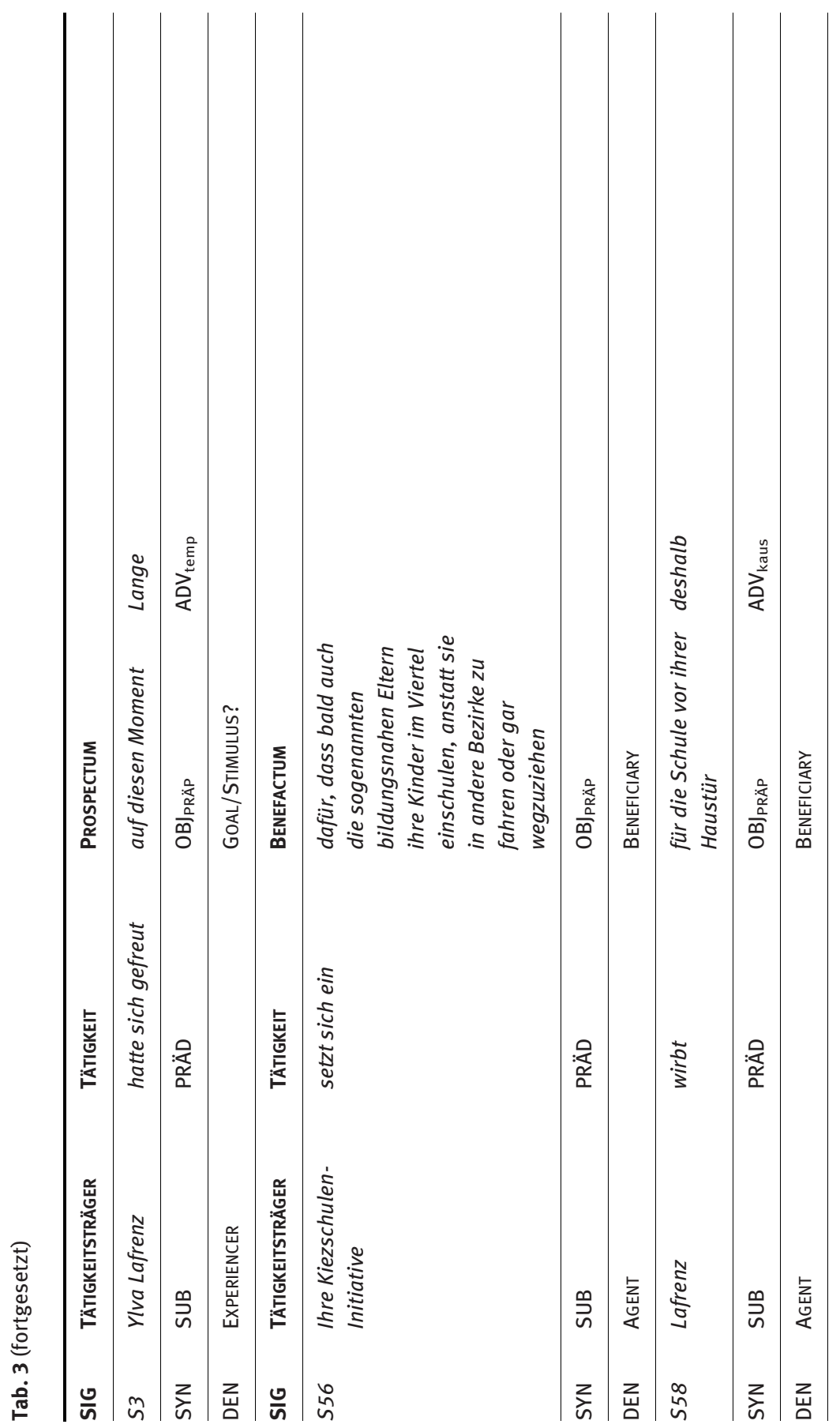




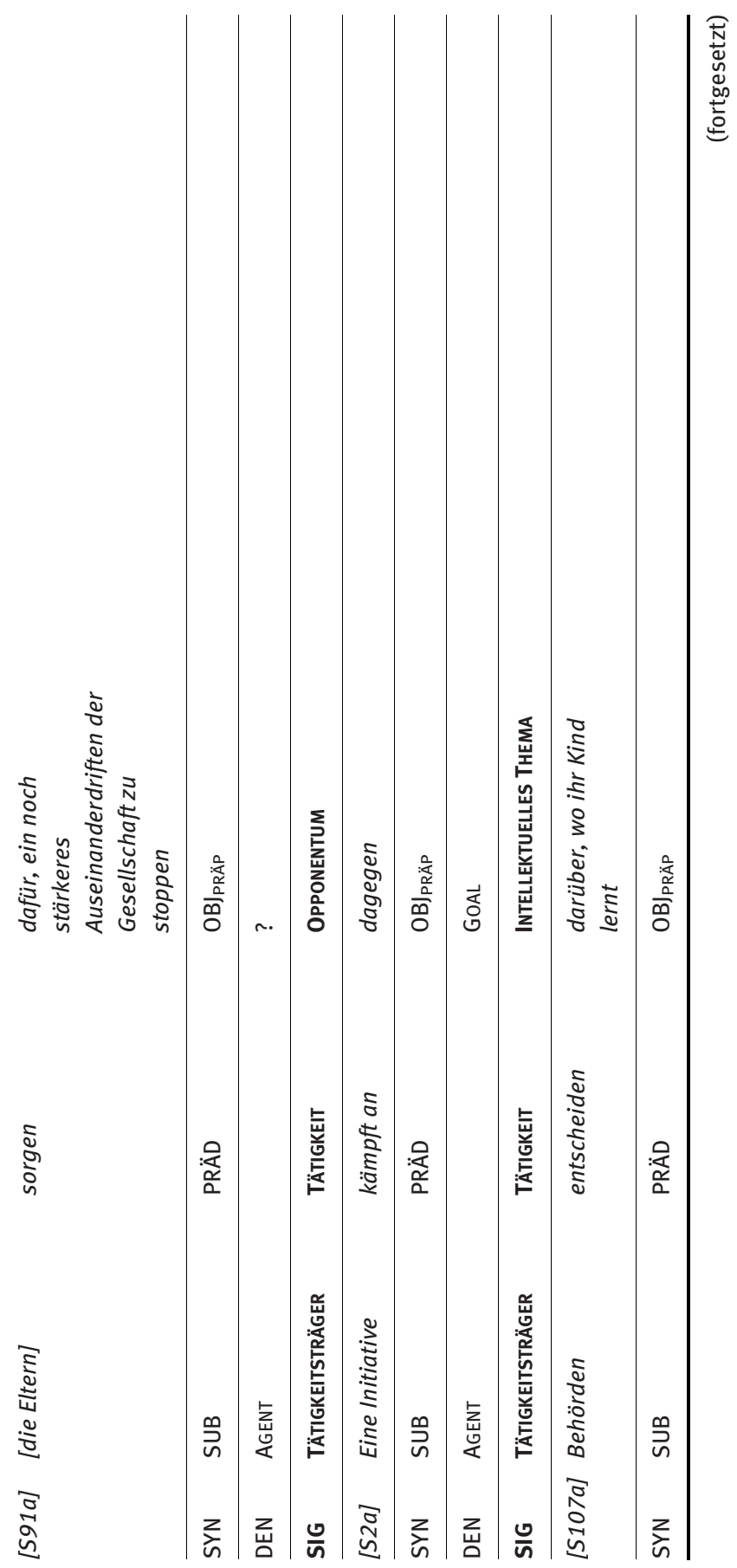




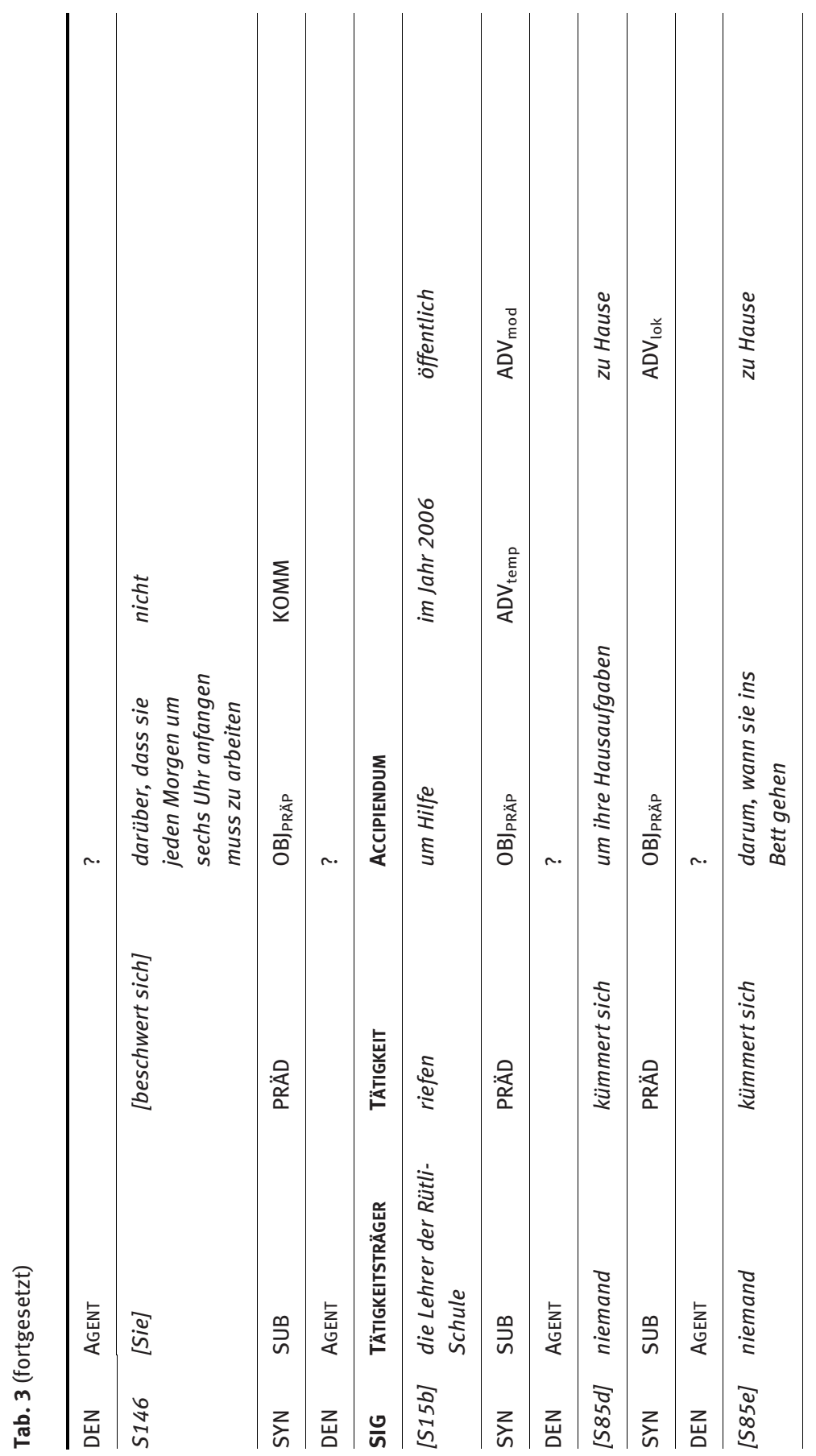




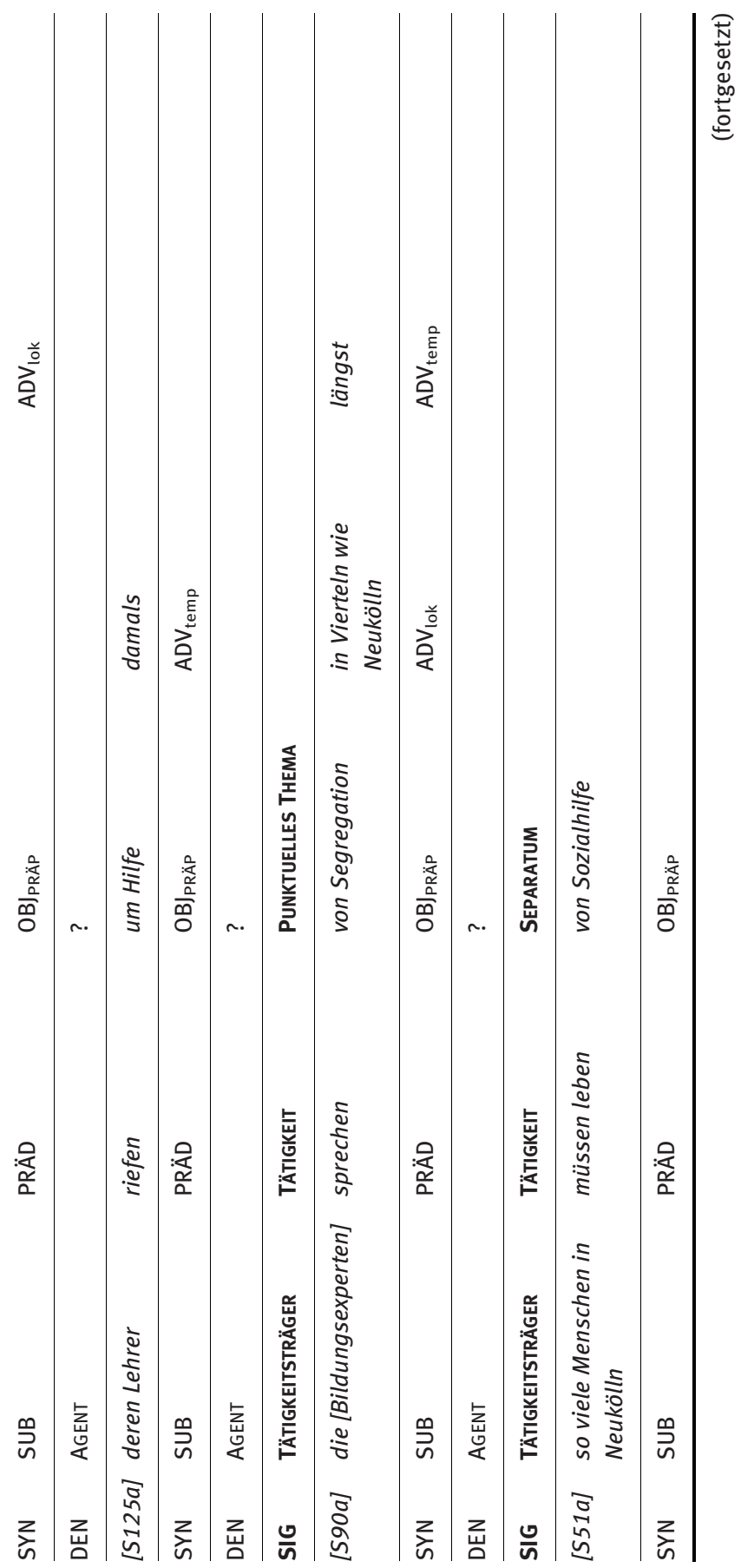




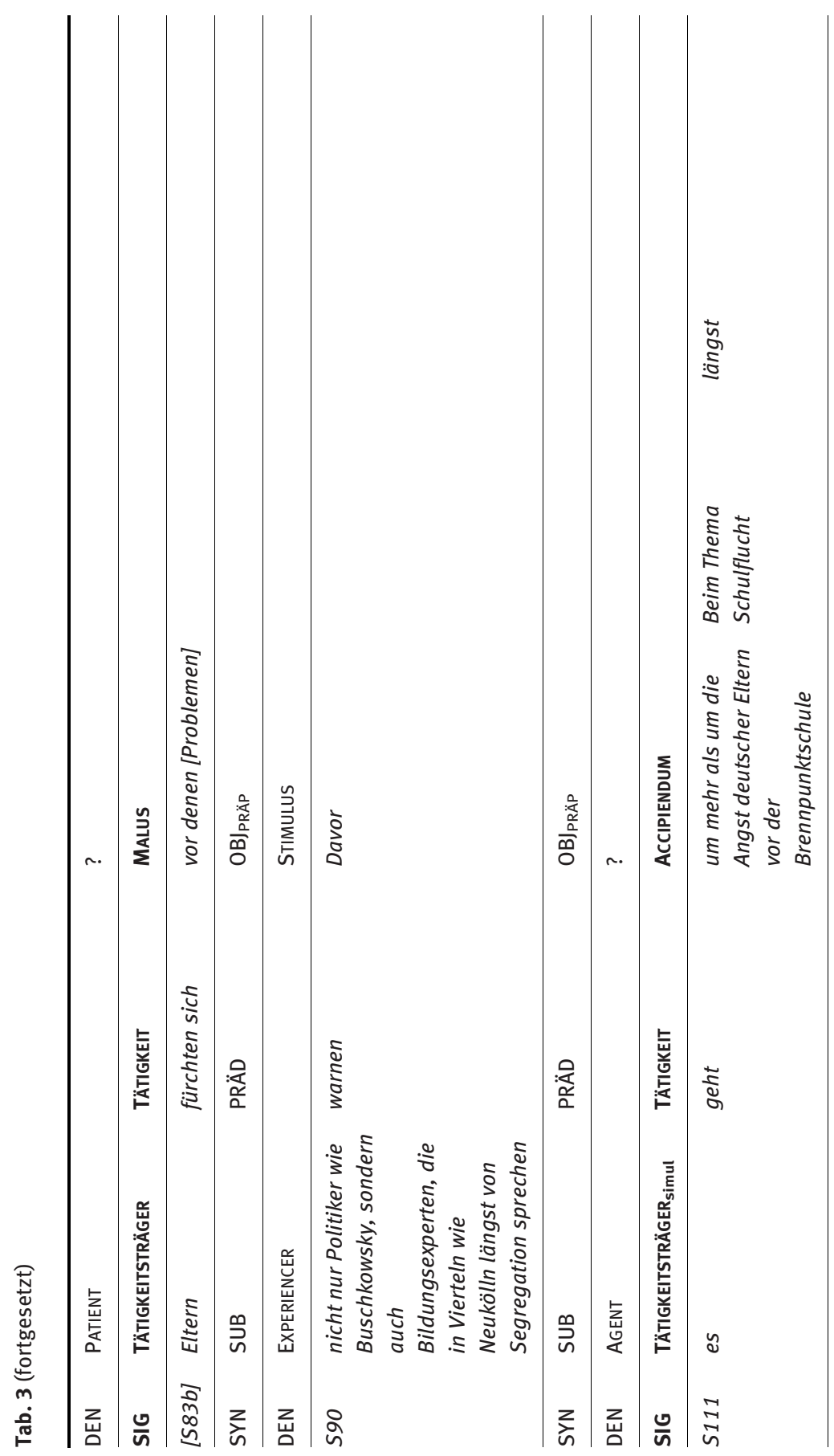




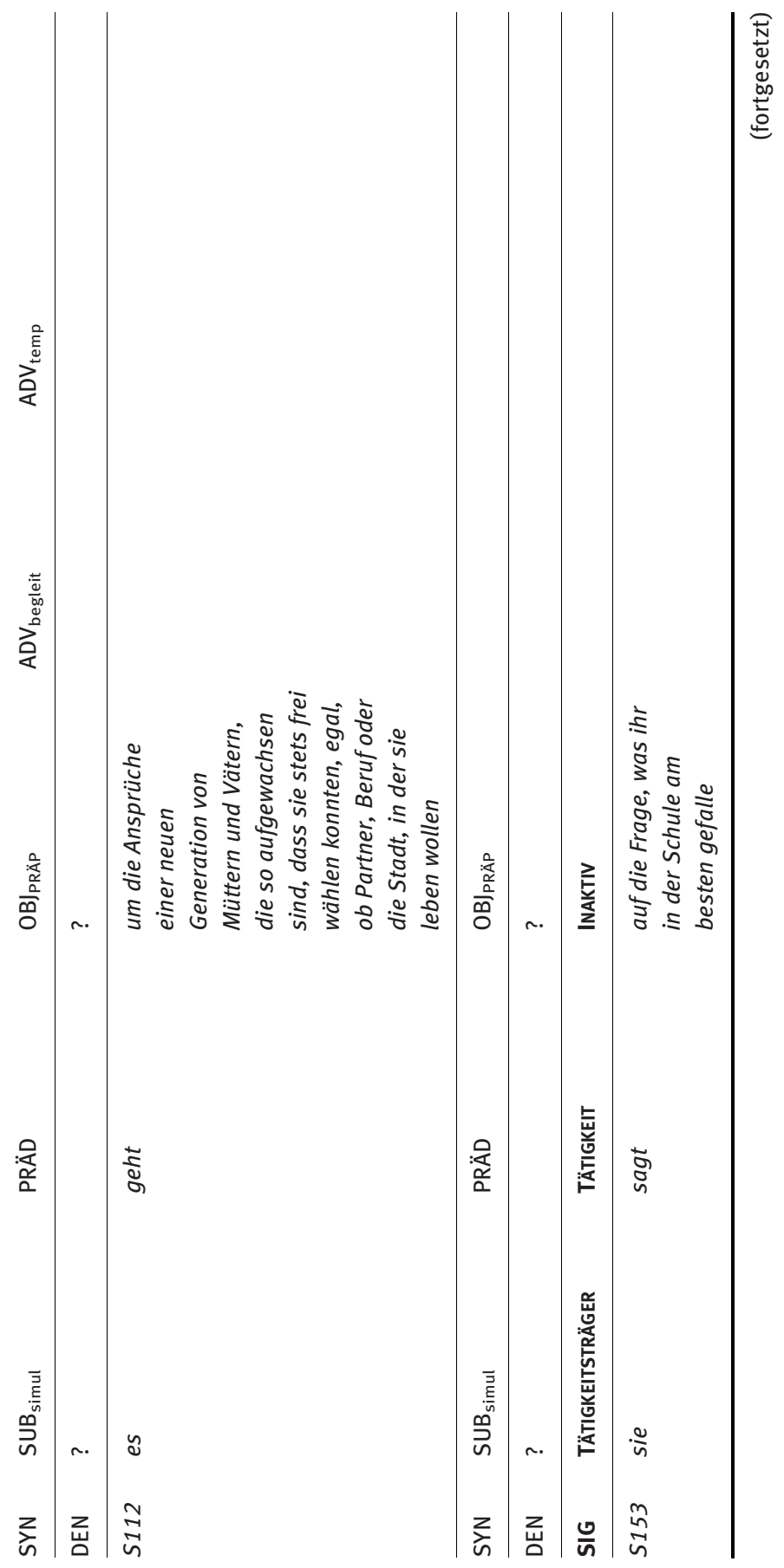




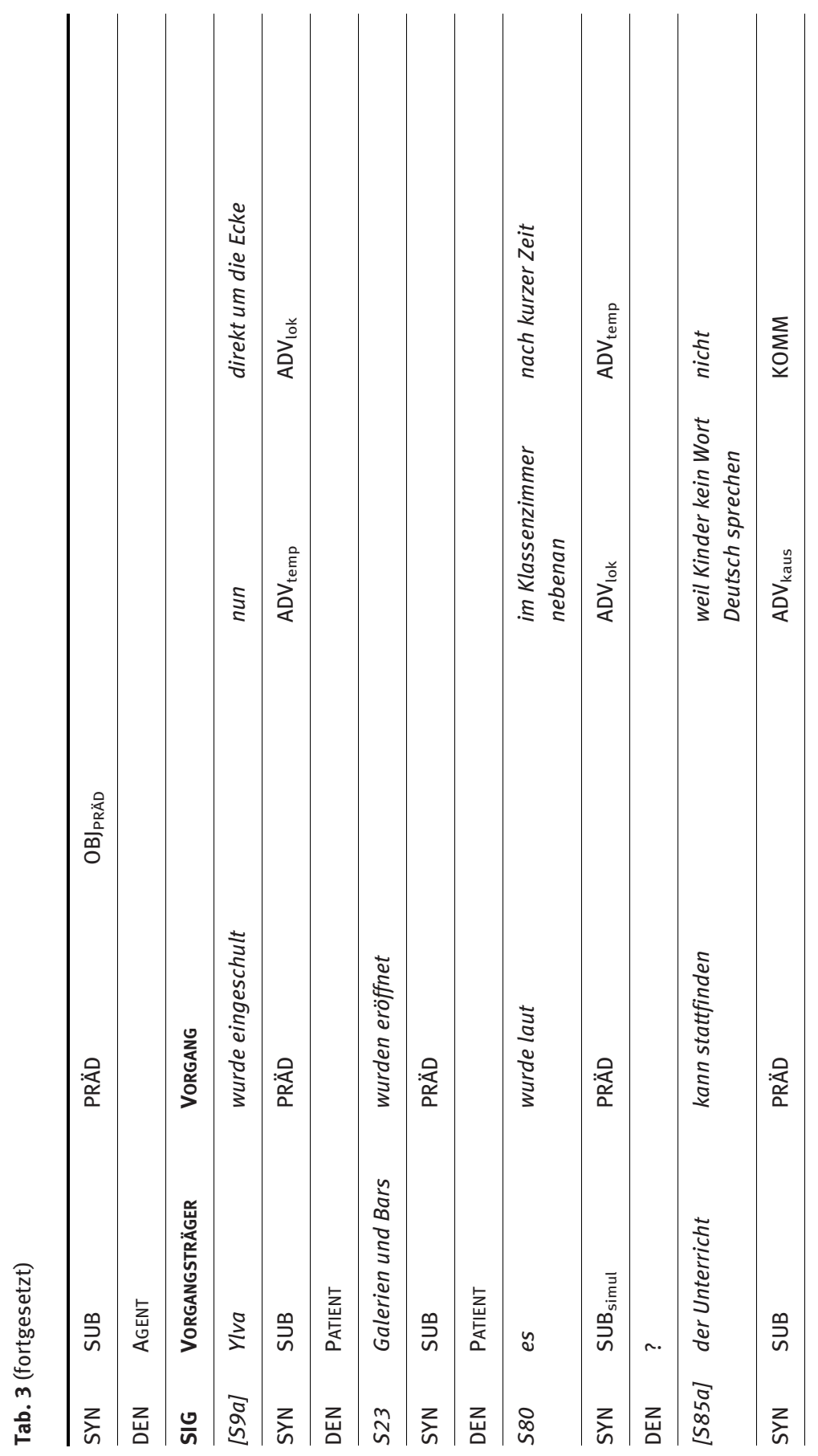




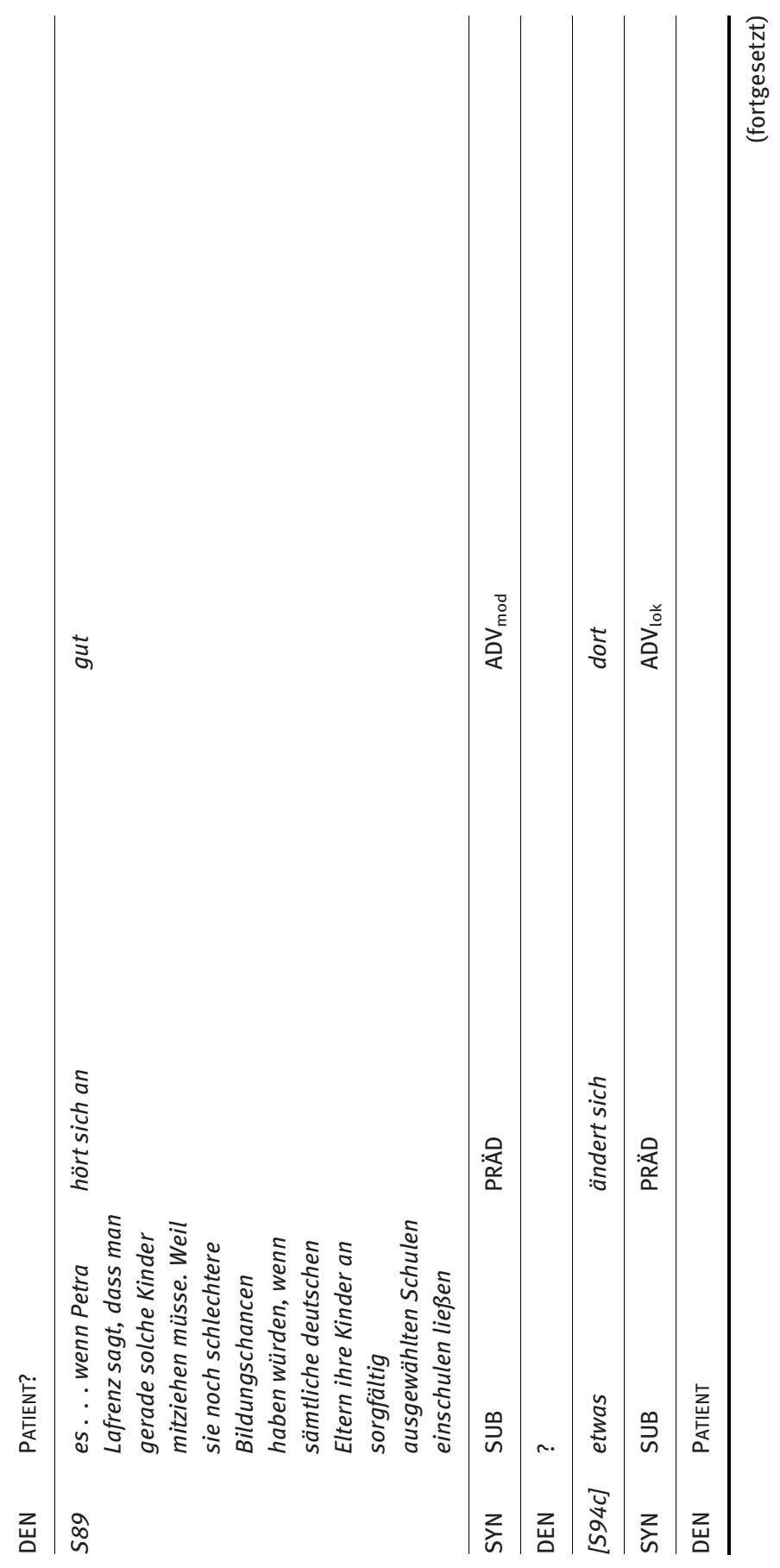




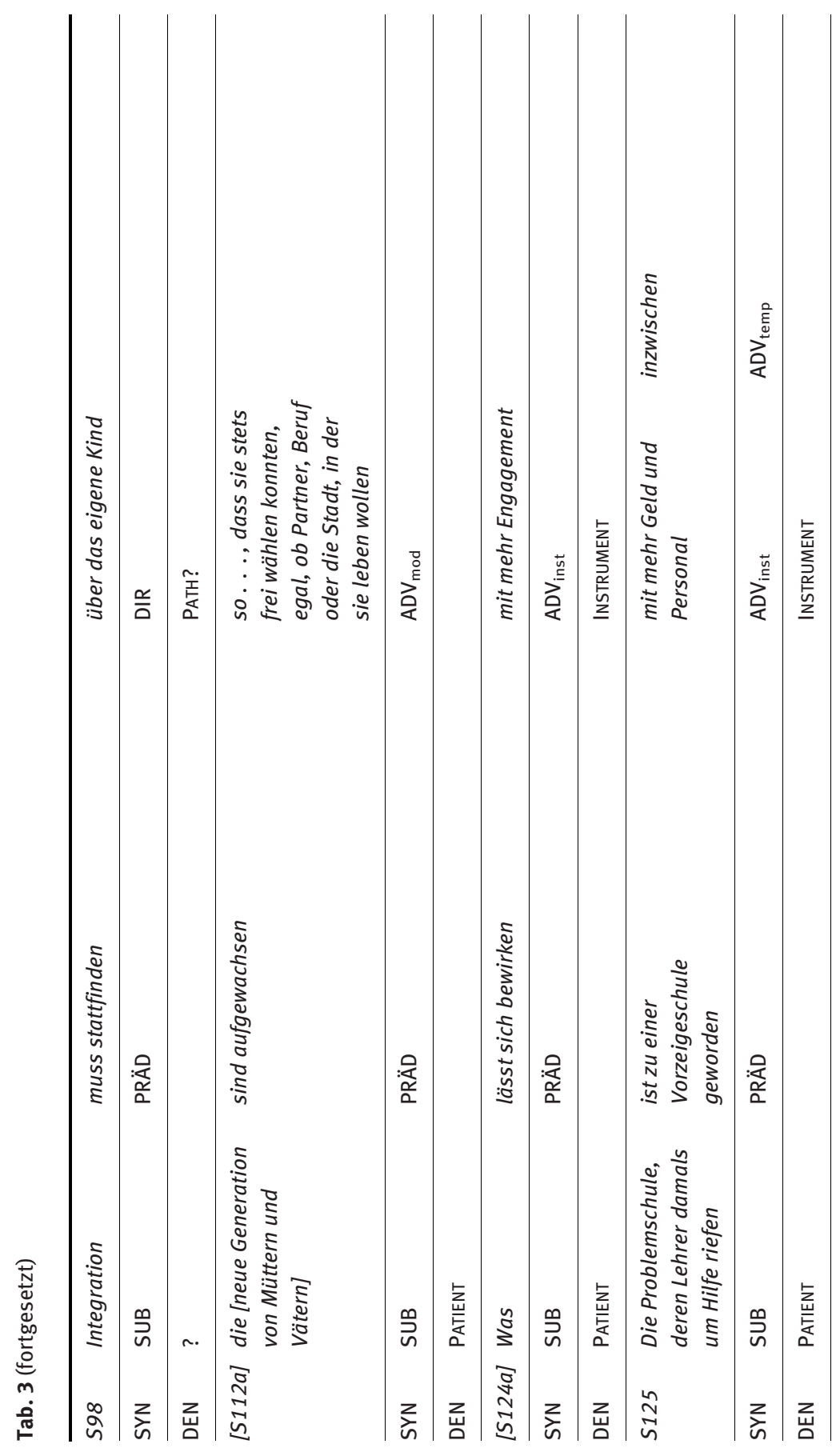




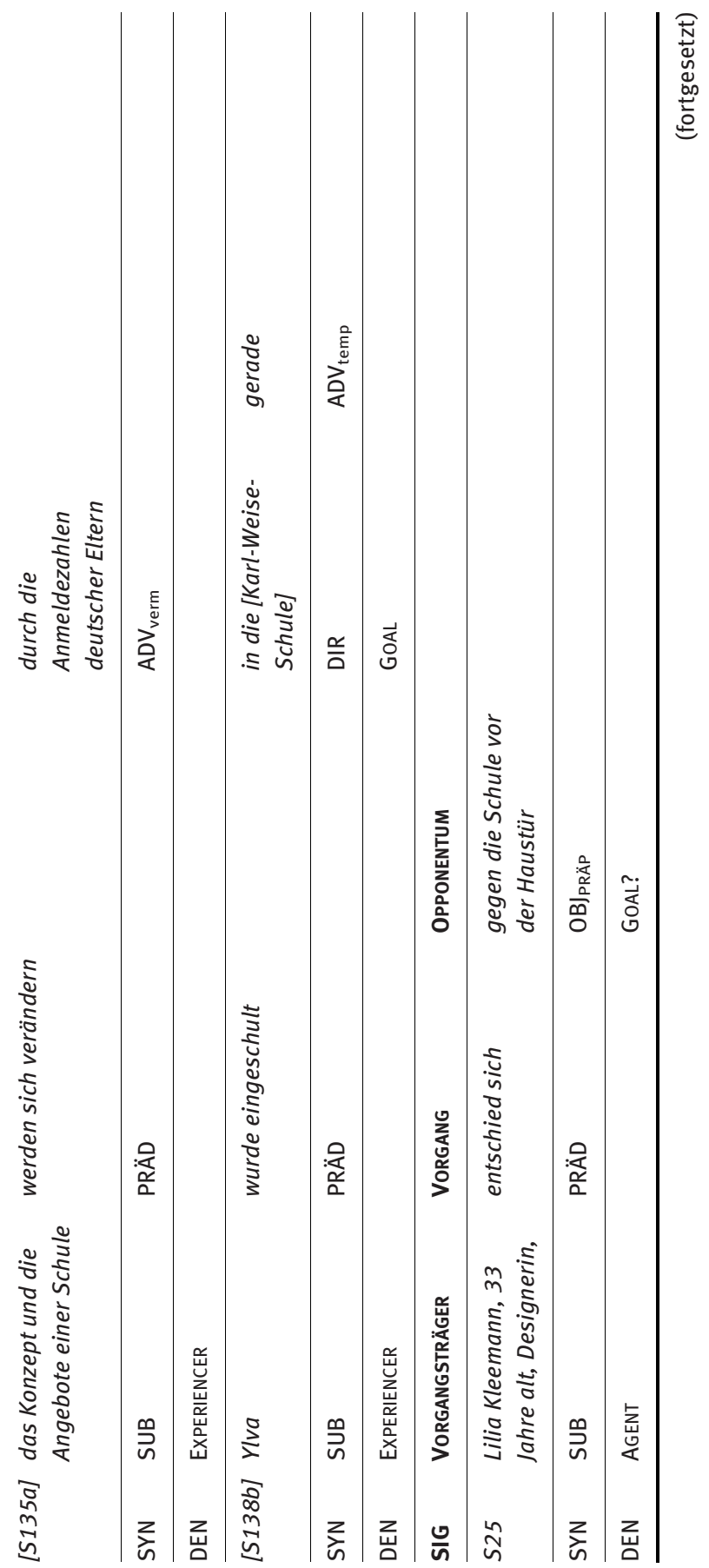




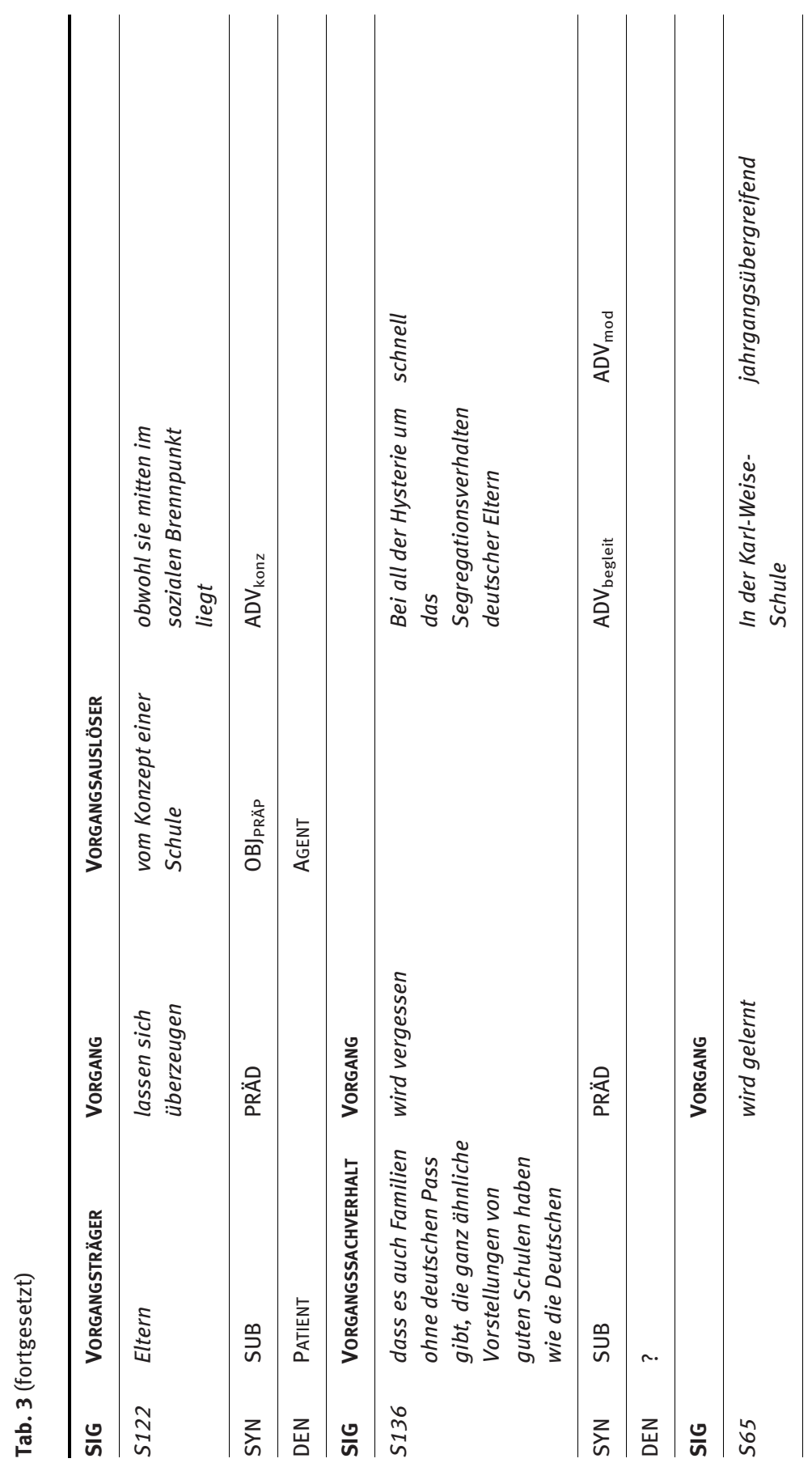




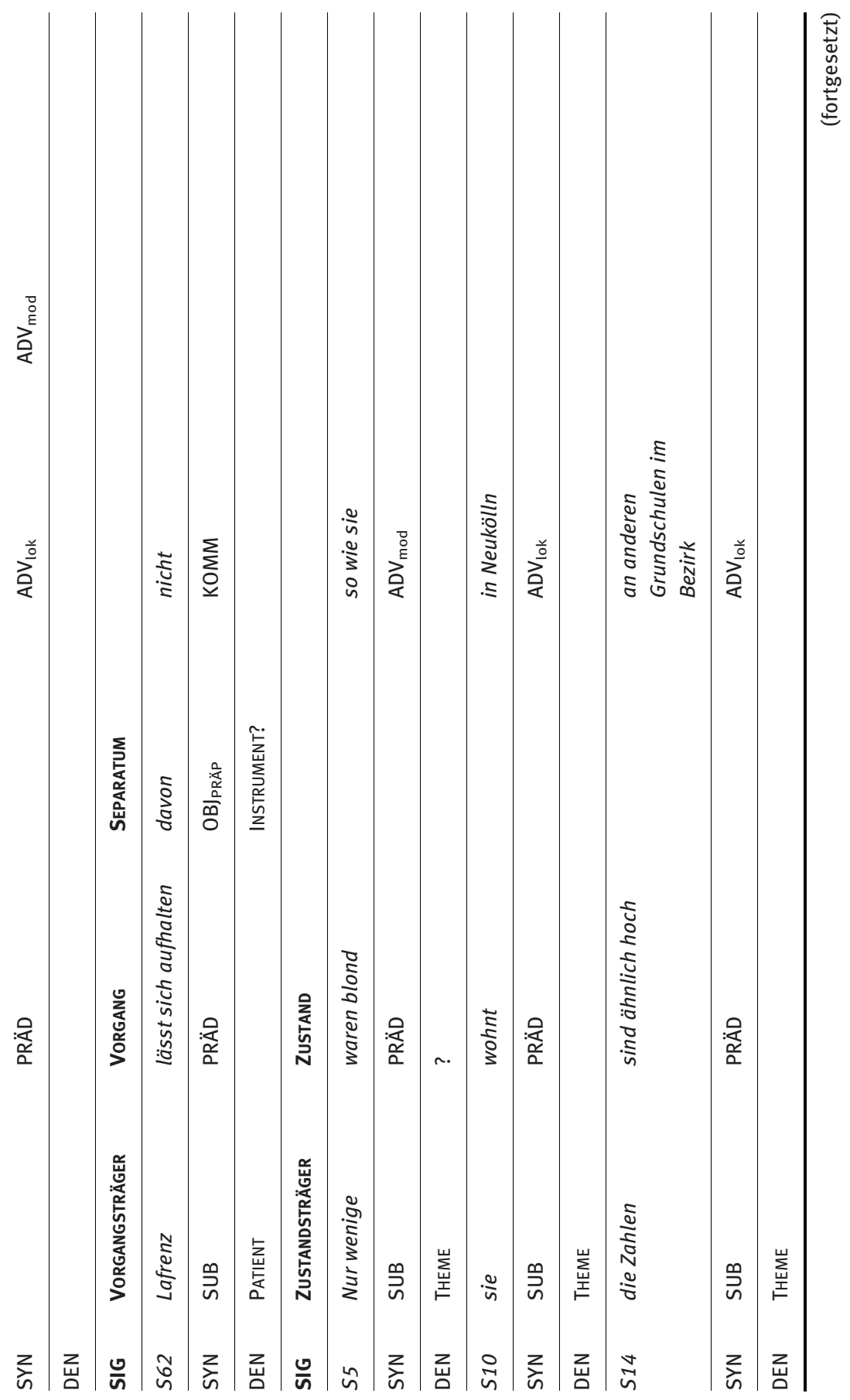




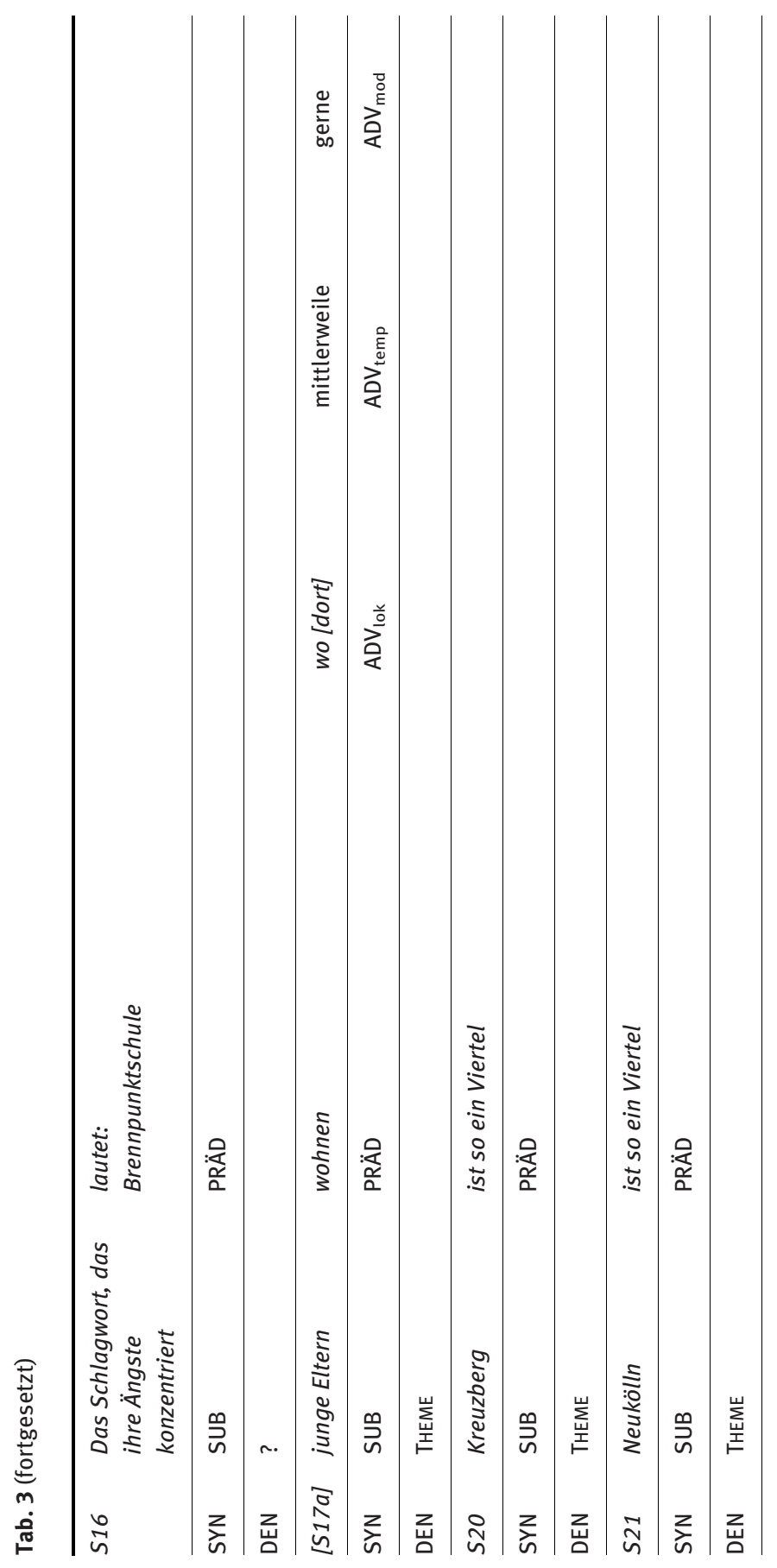




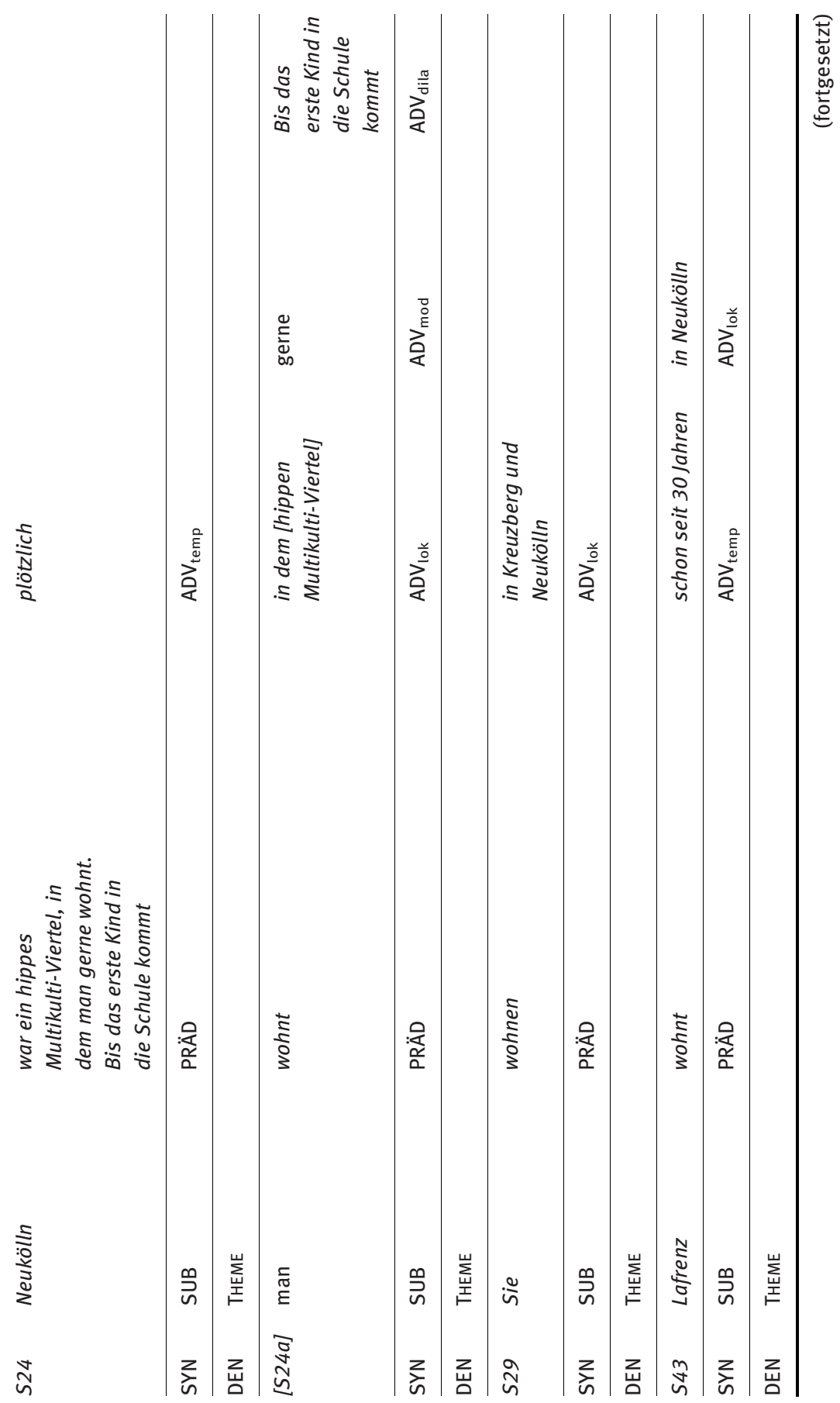




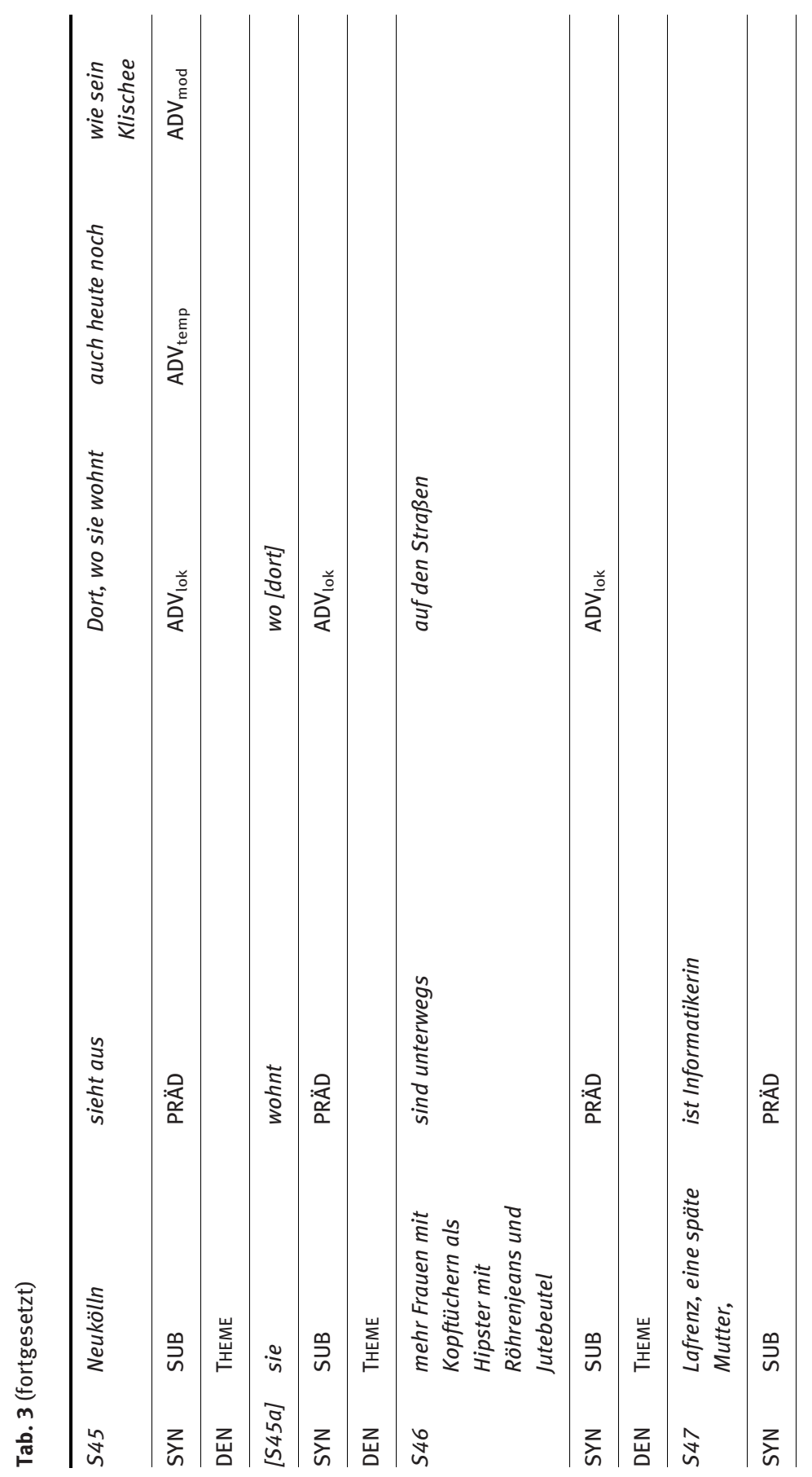




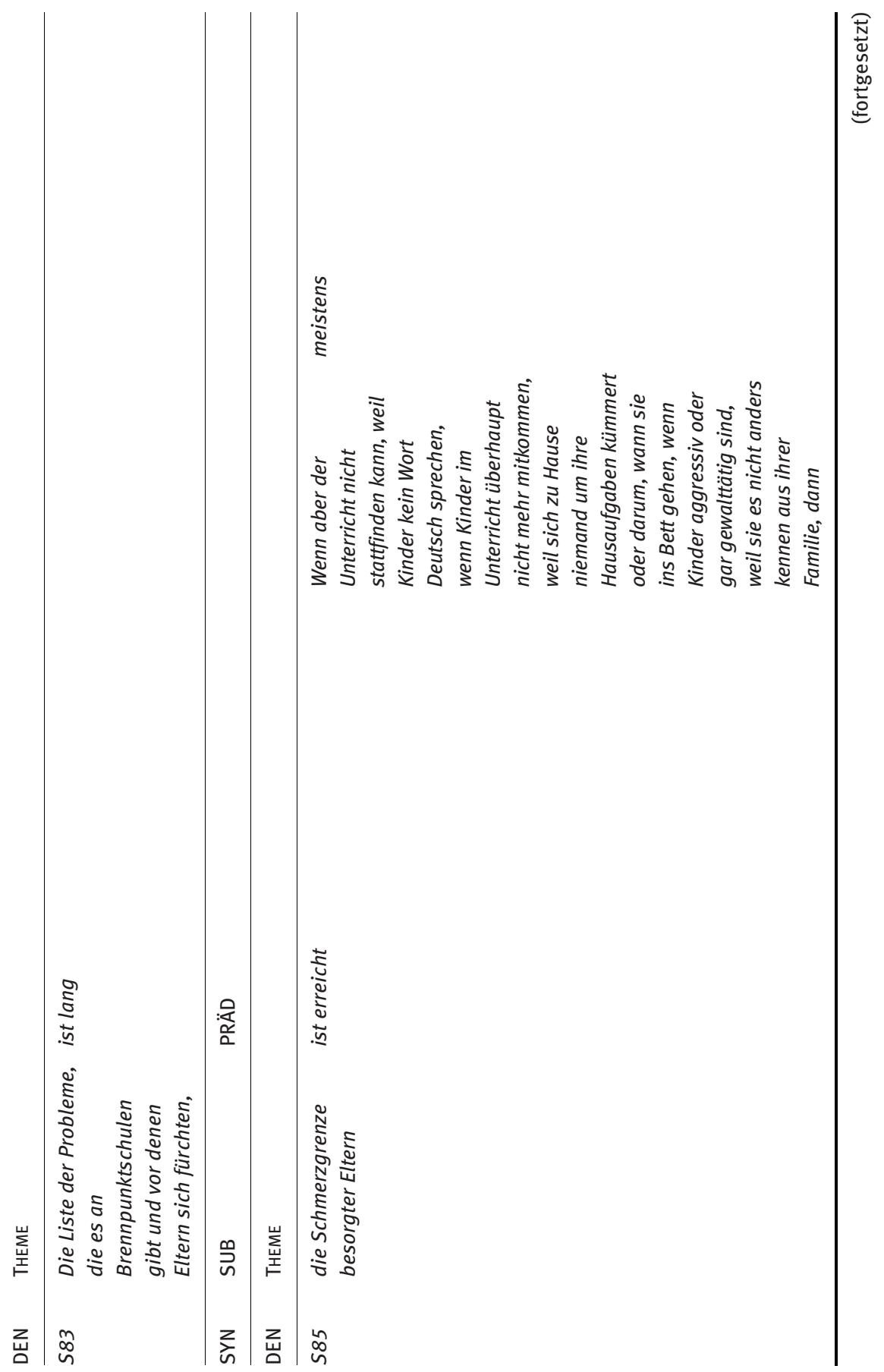


216 Vilmos Ágel \& Dagobert Höllein

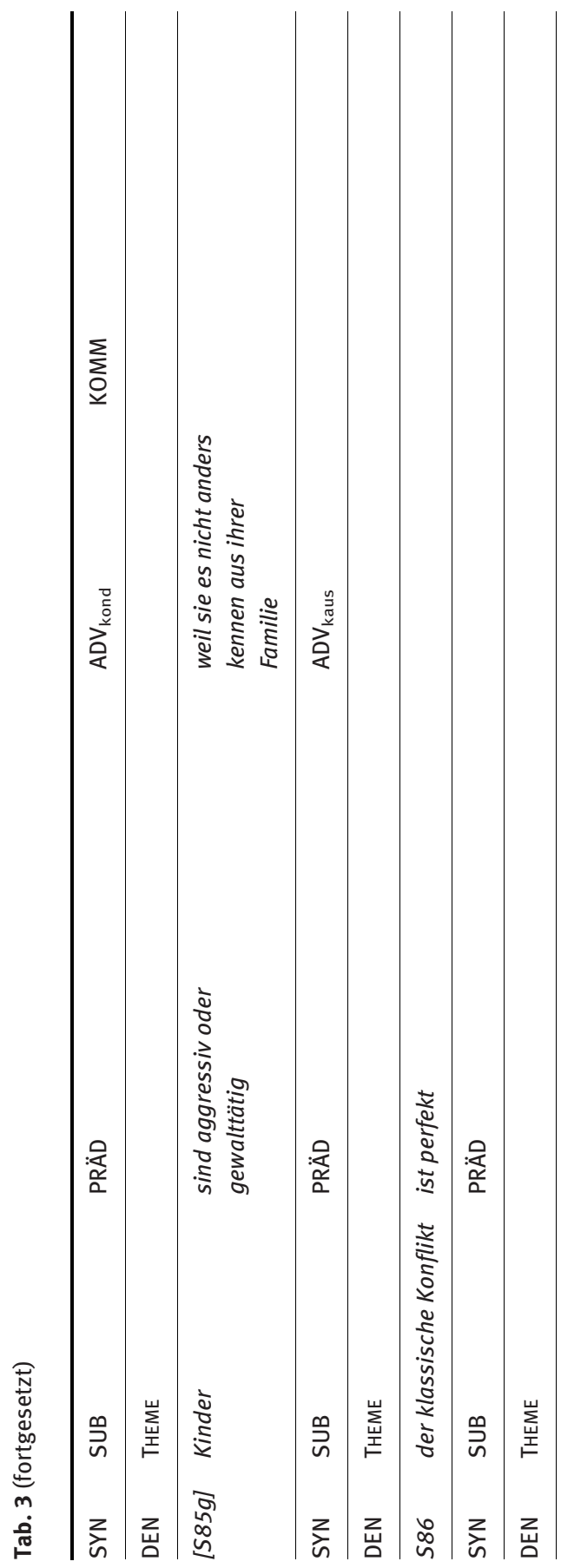




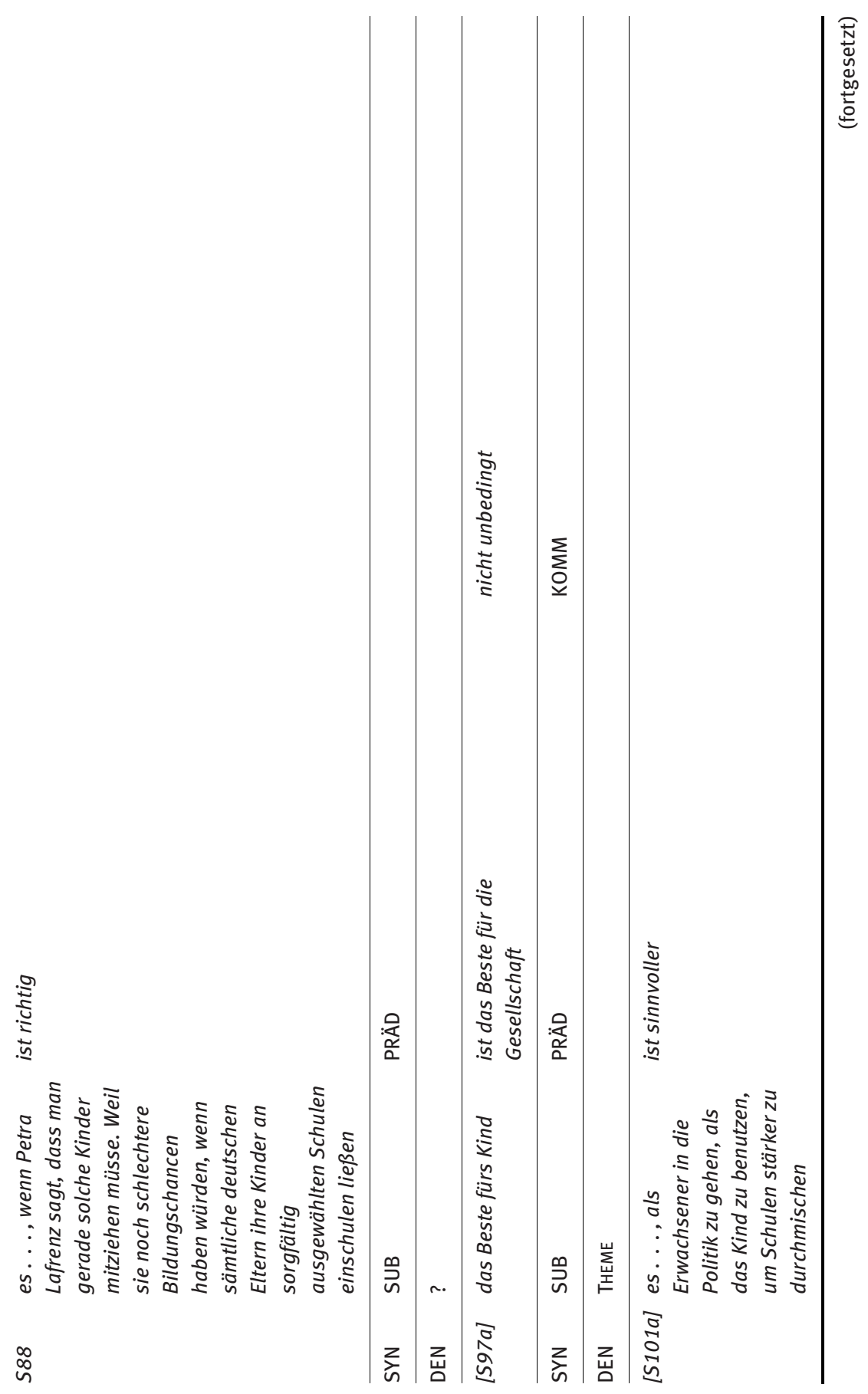




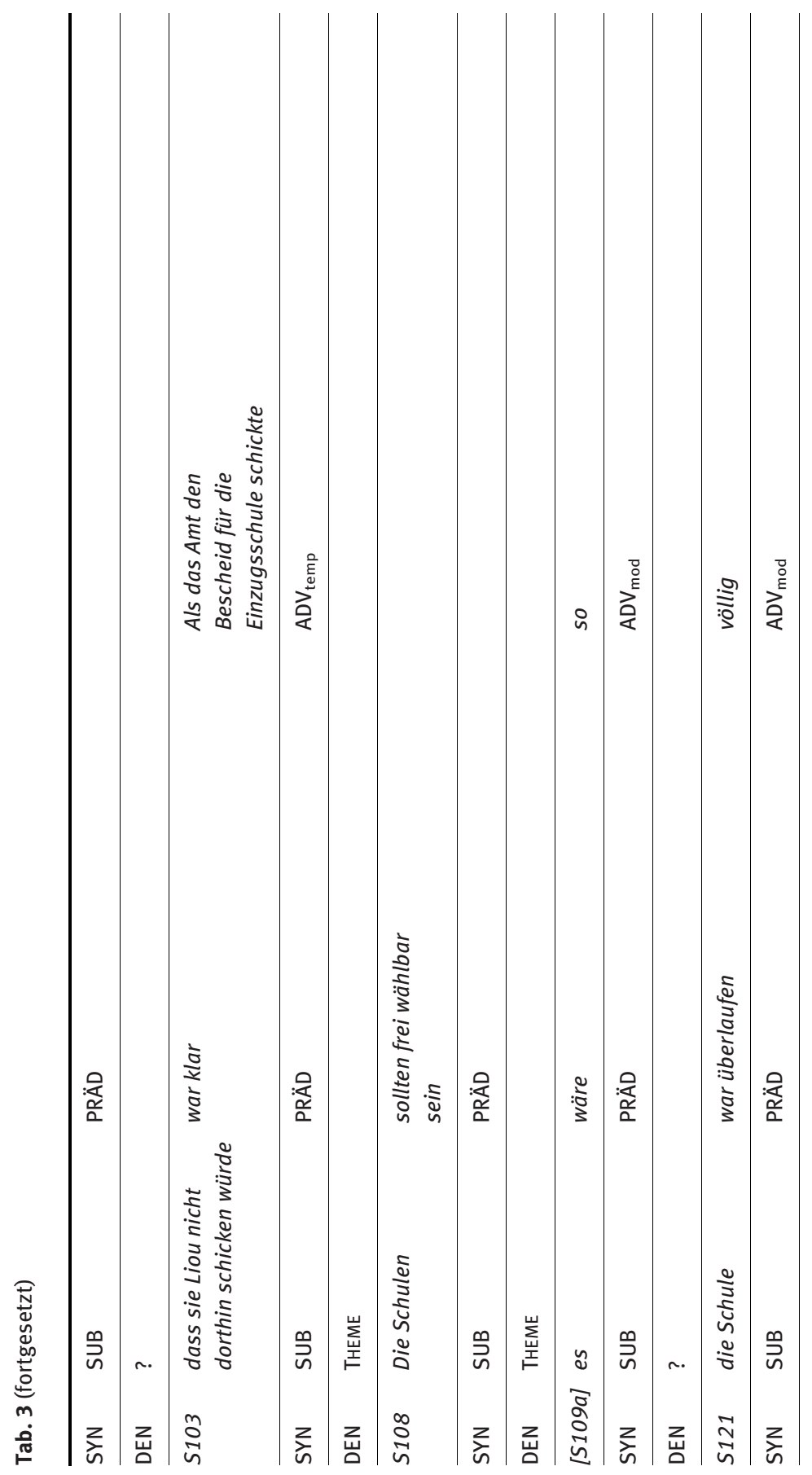




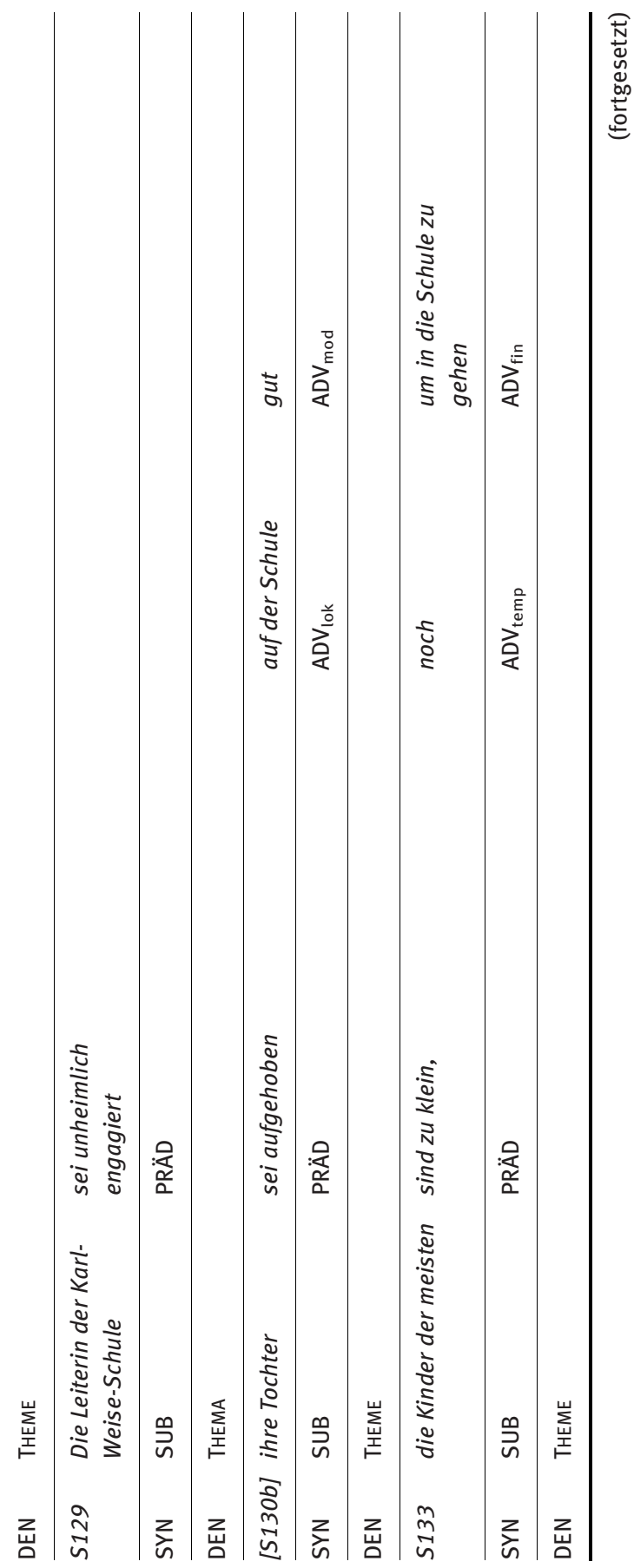




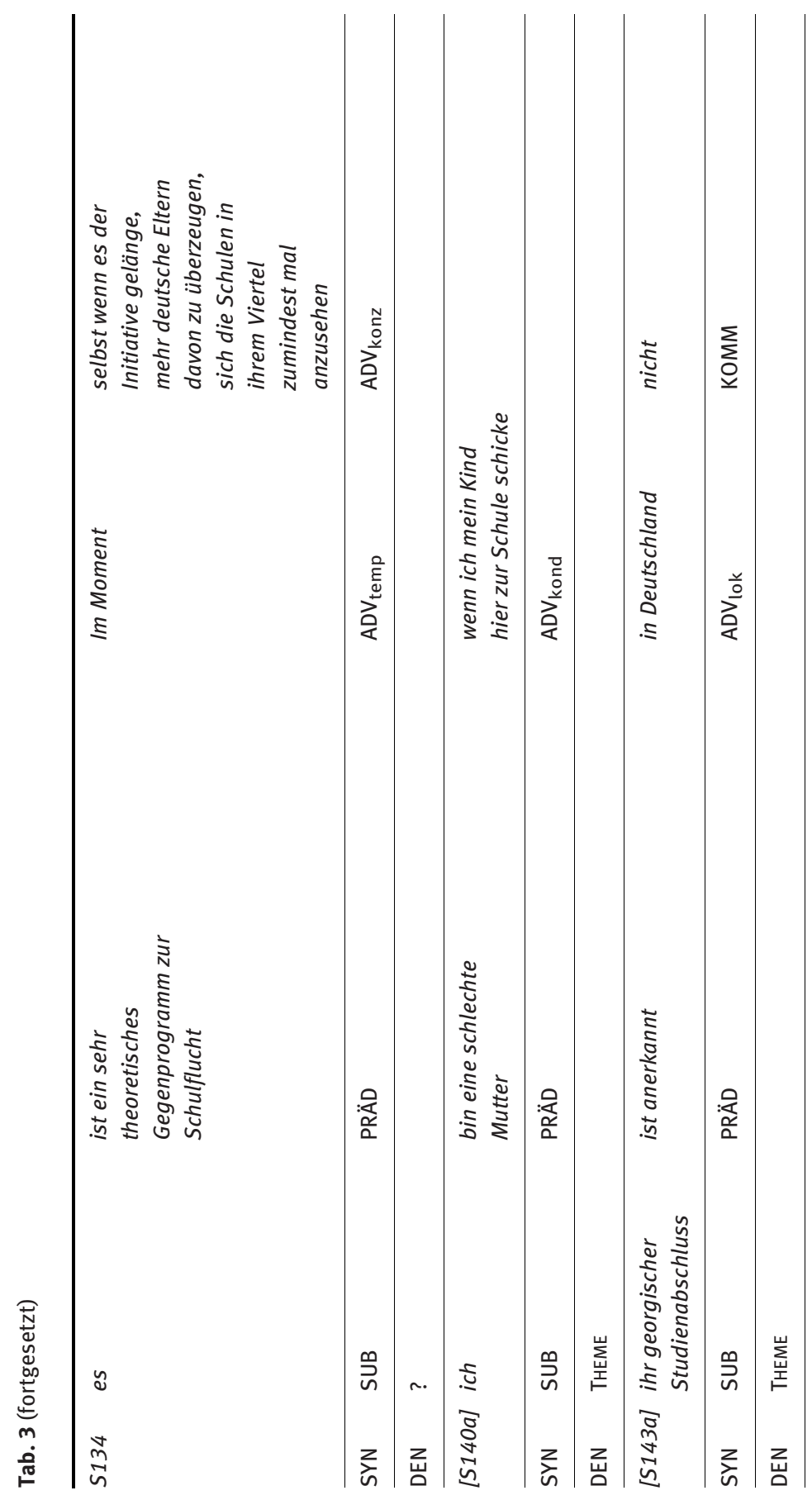




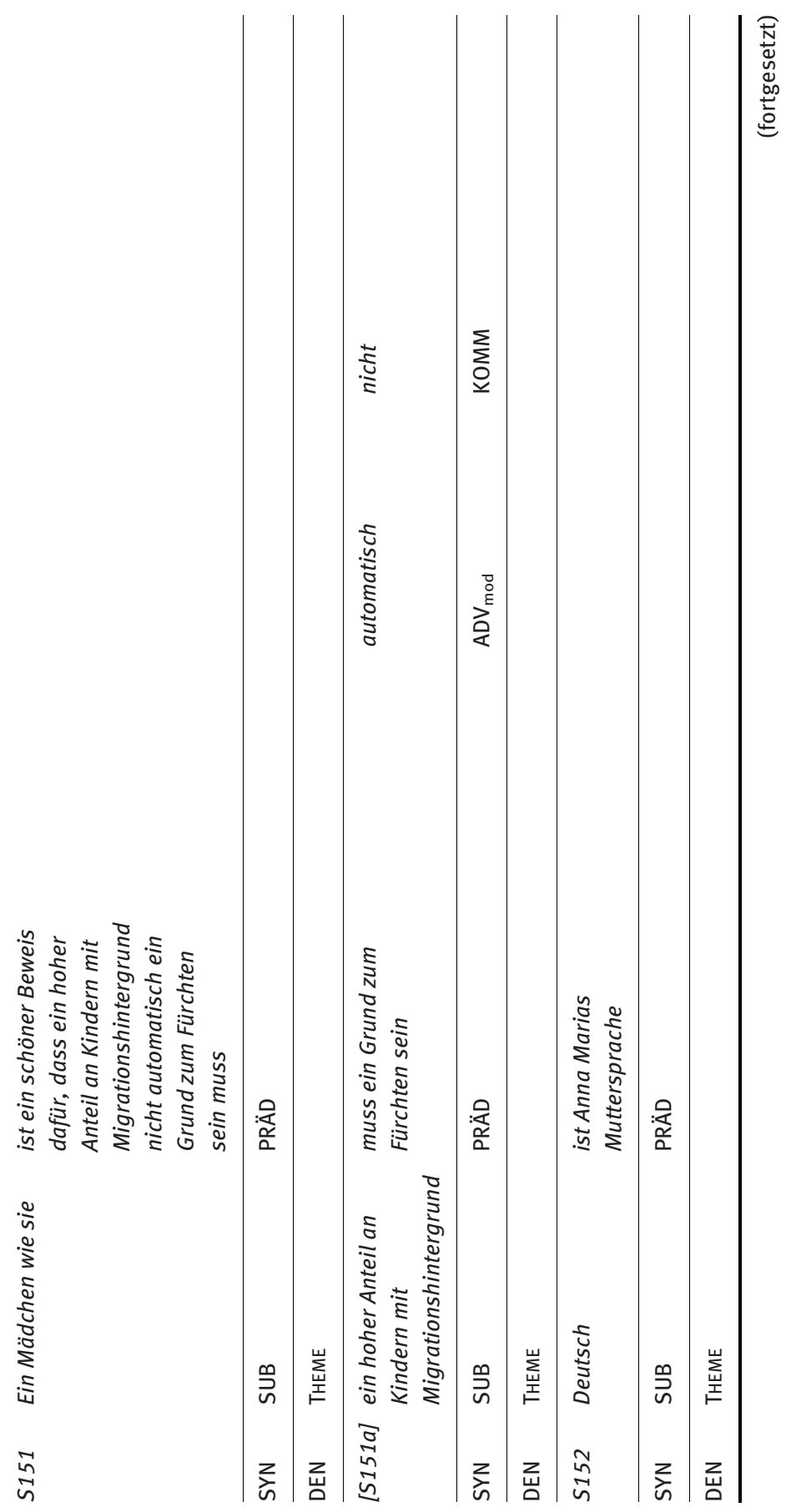




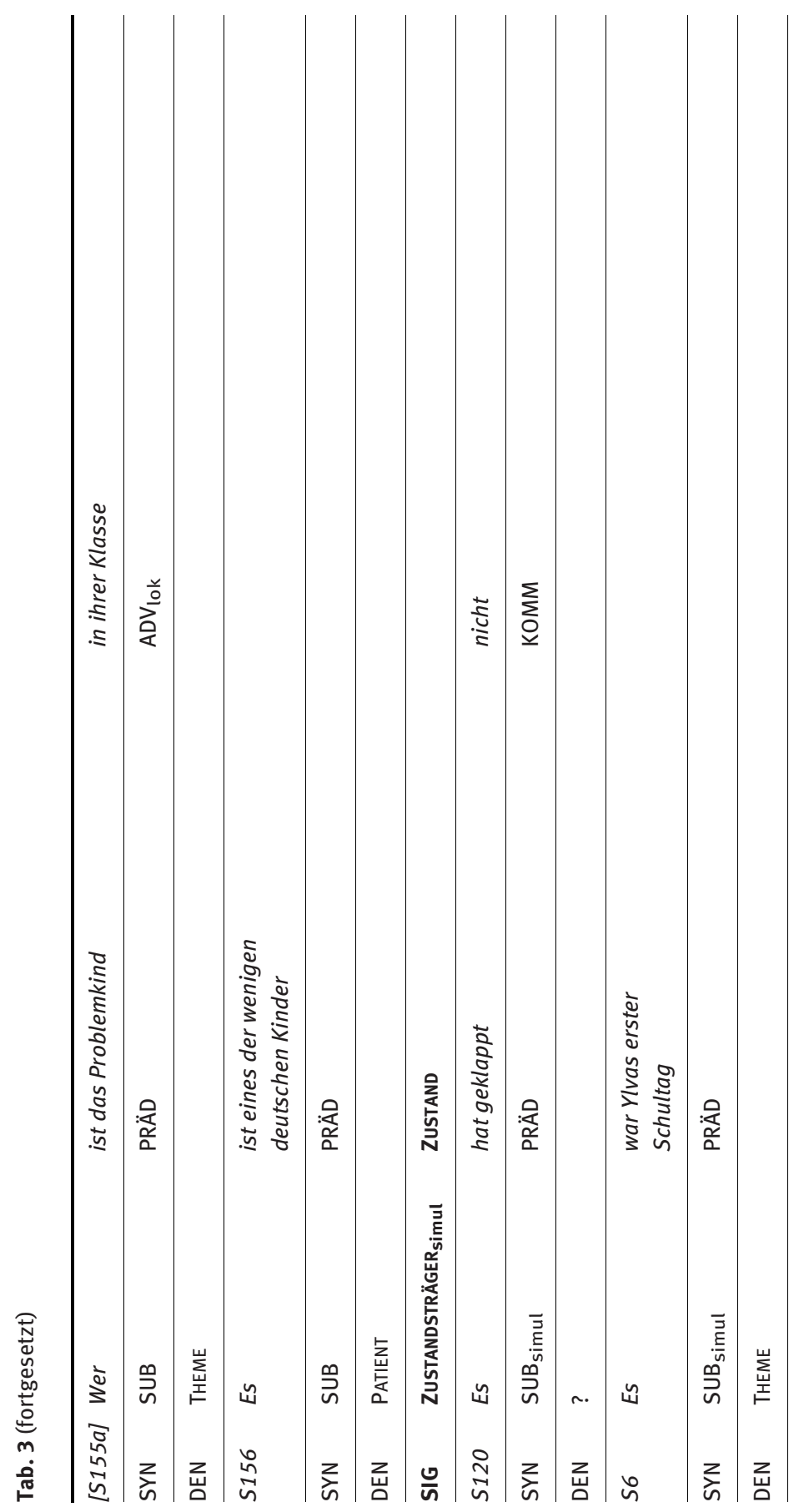




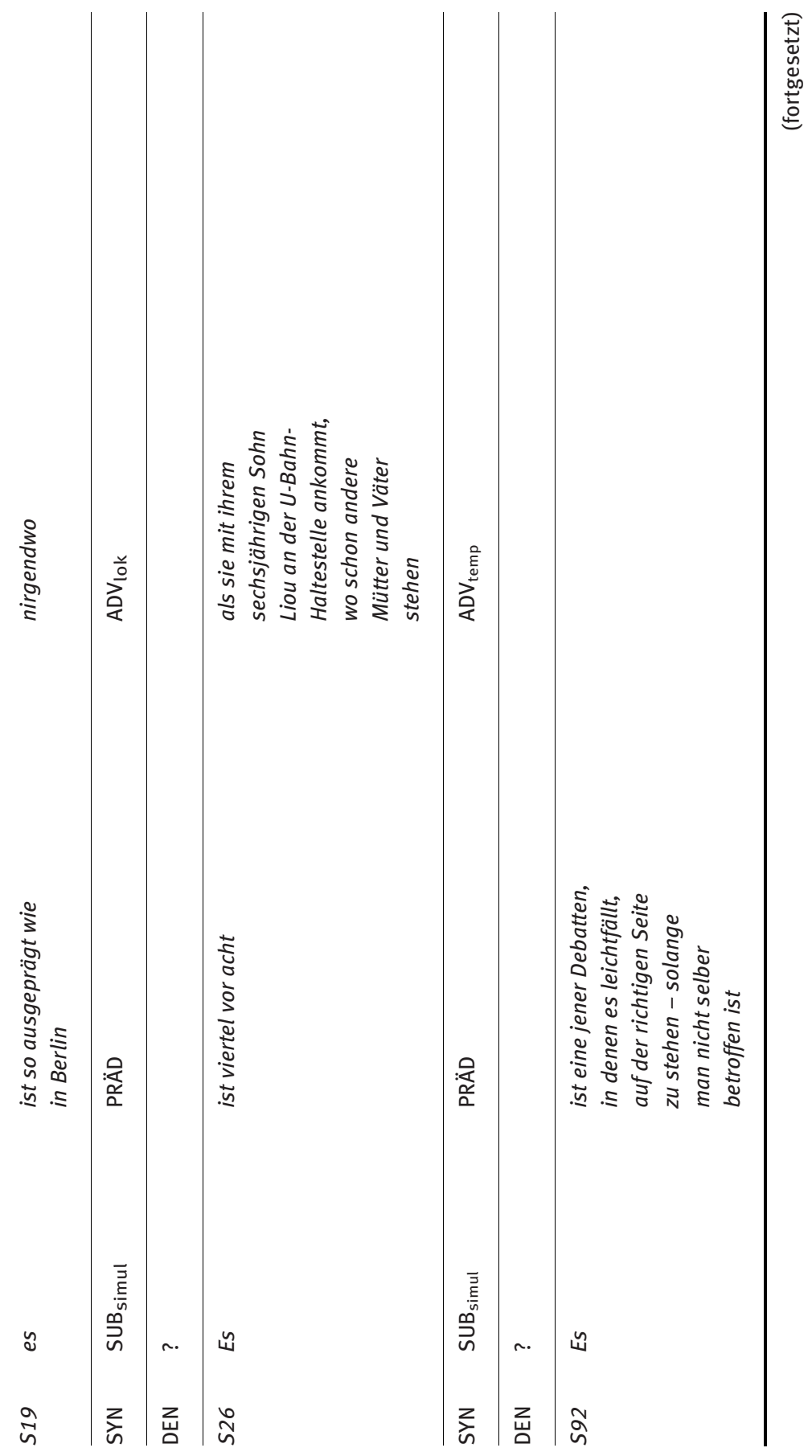




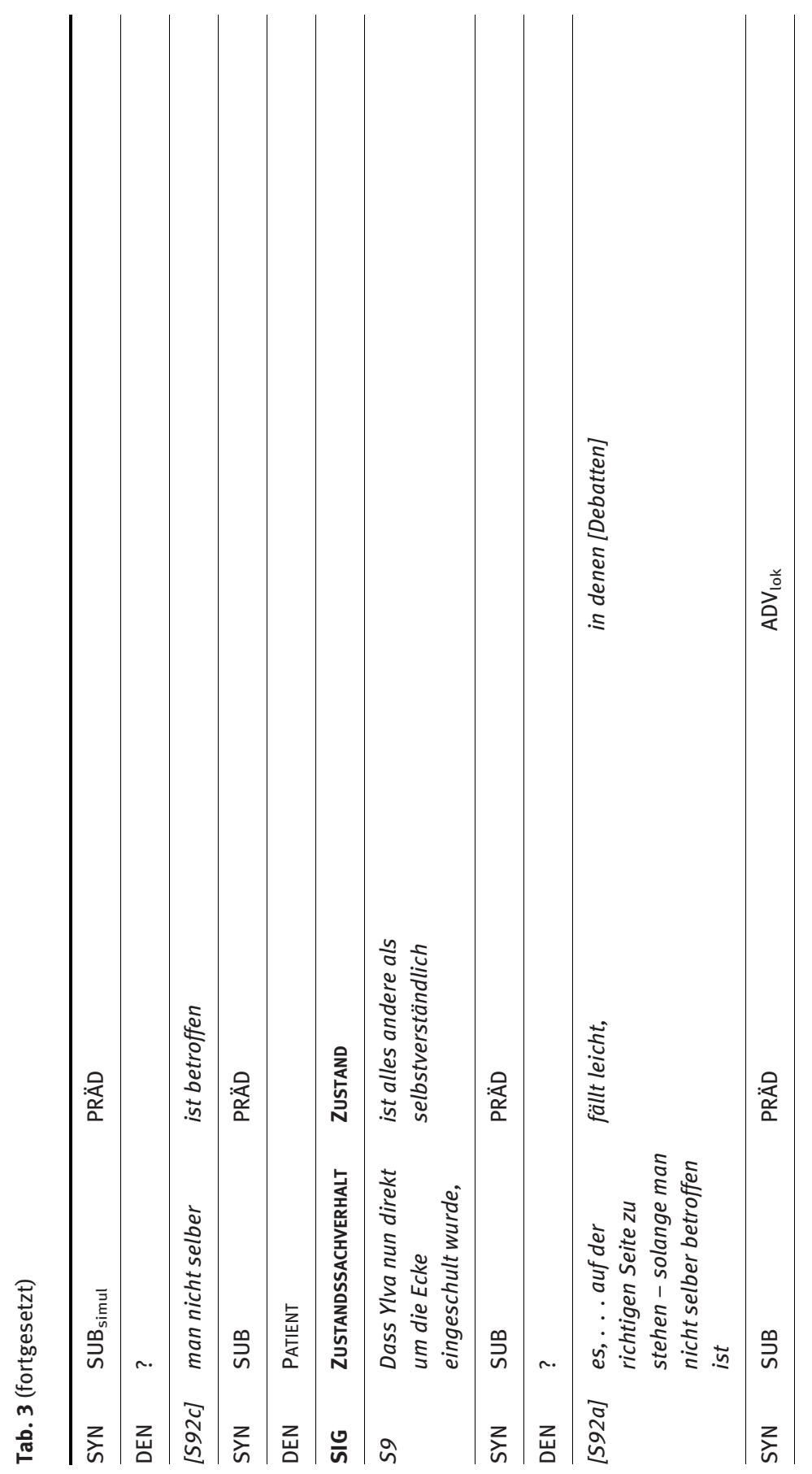




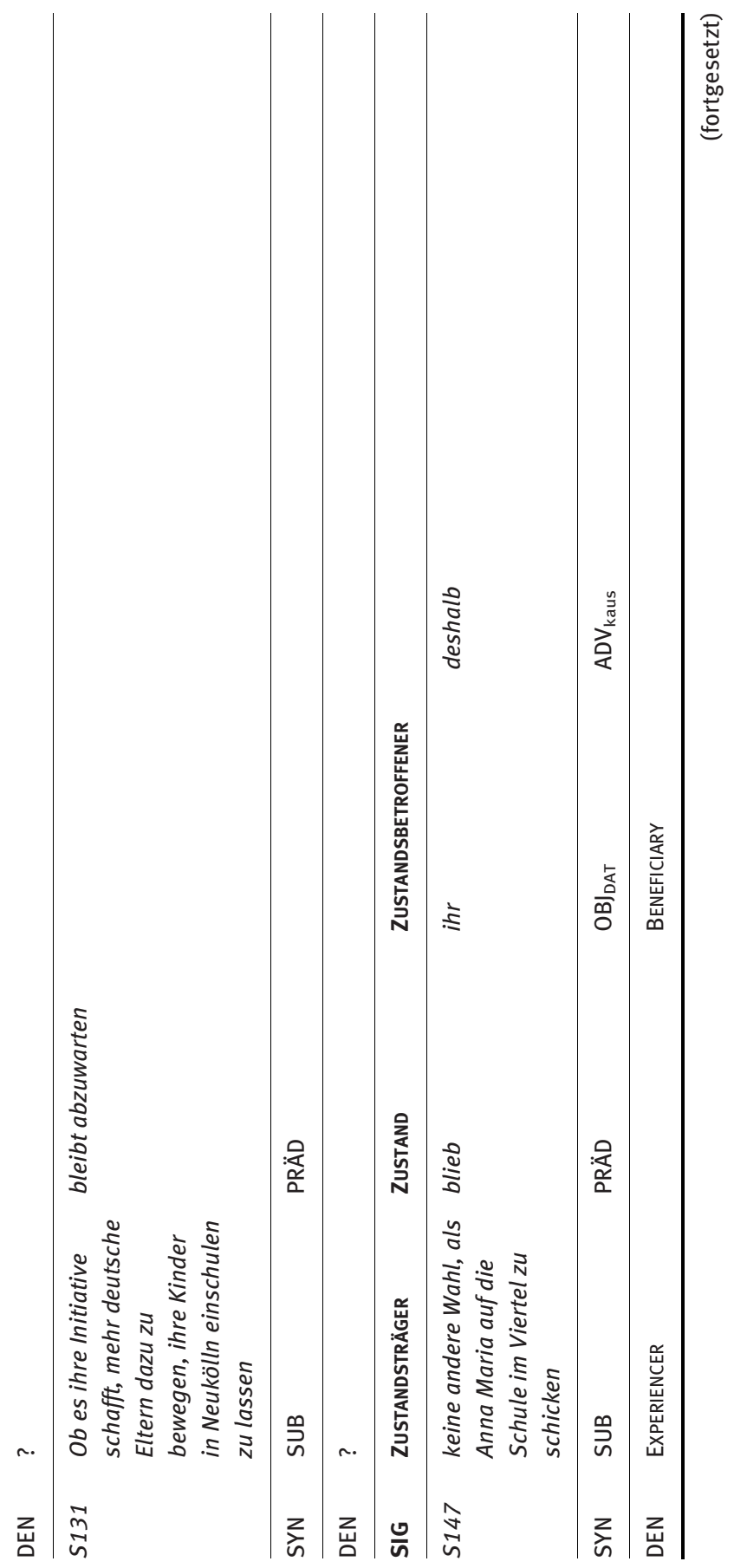




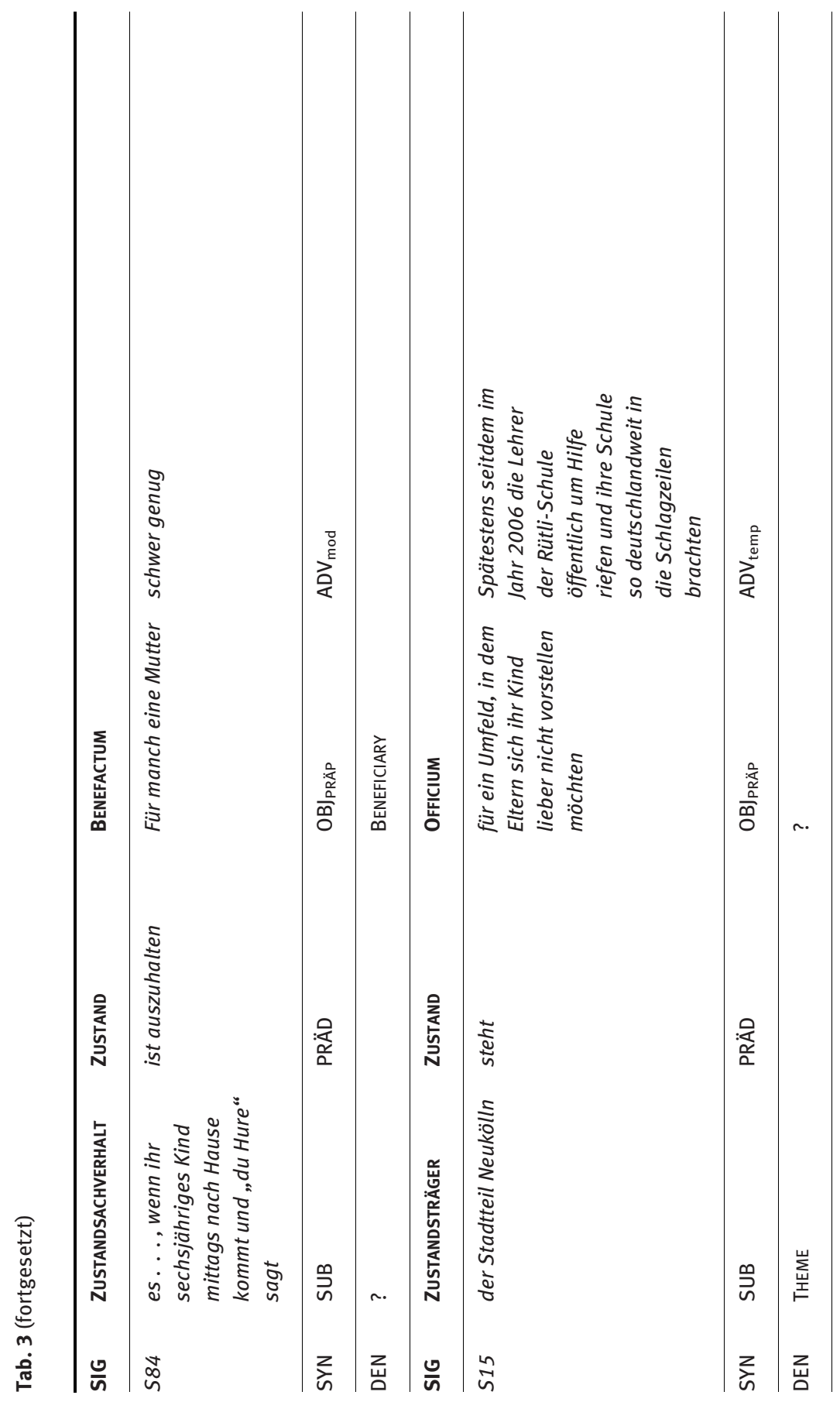




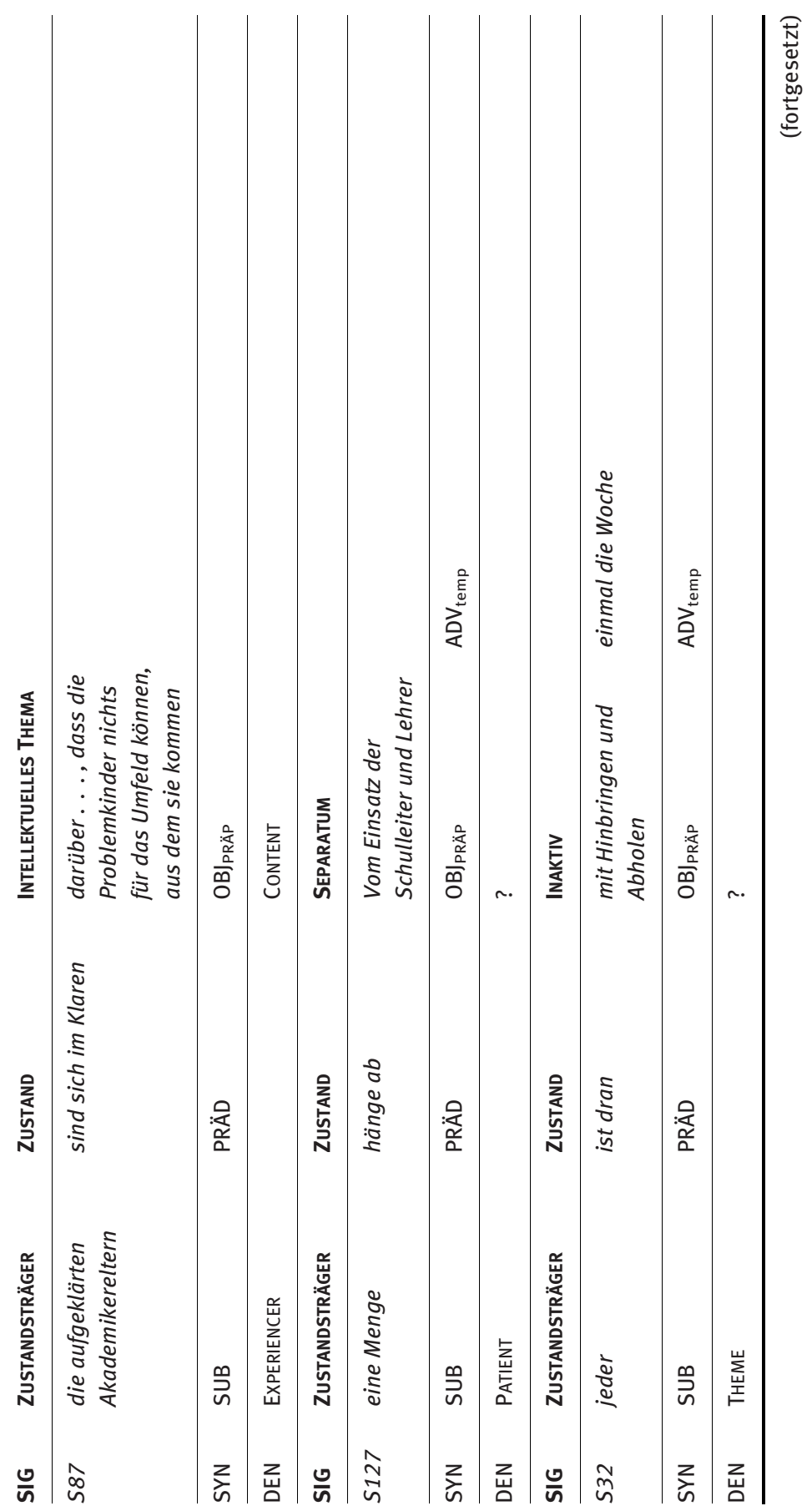




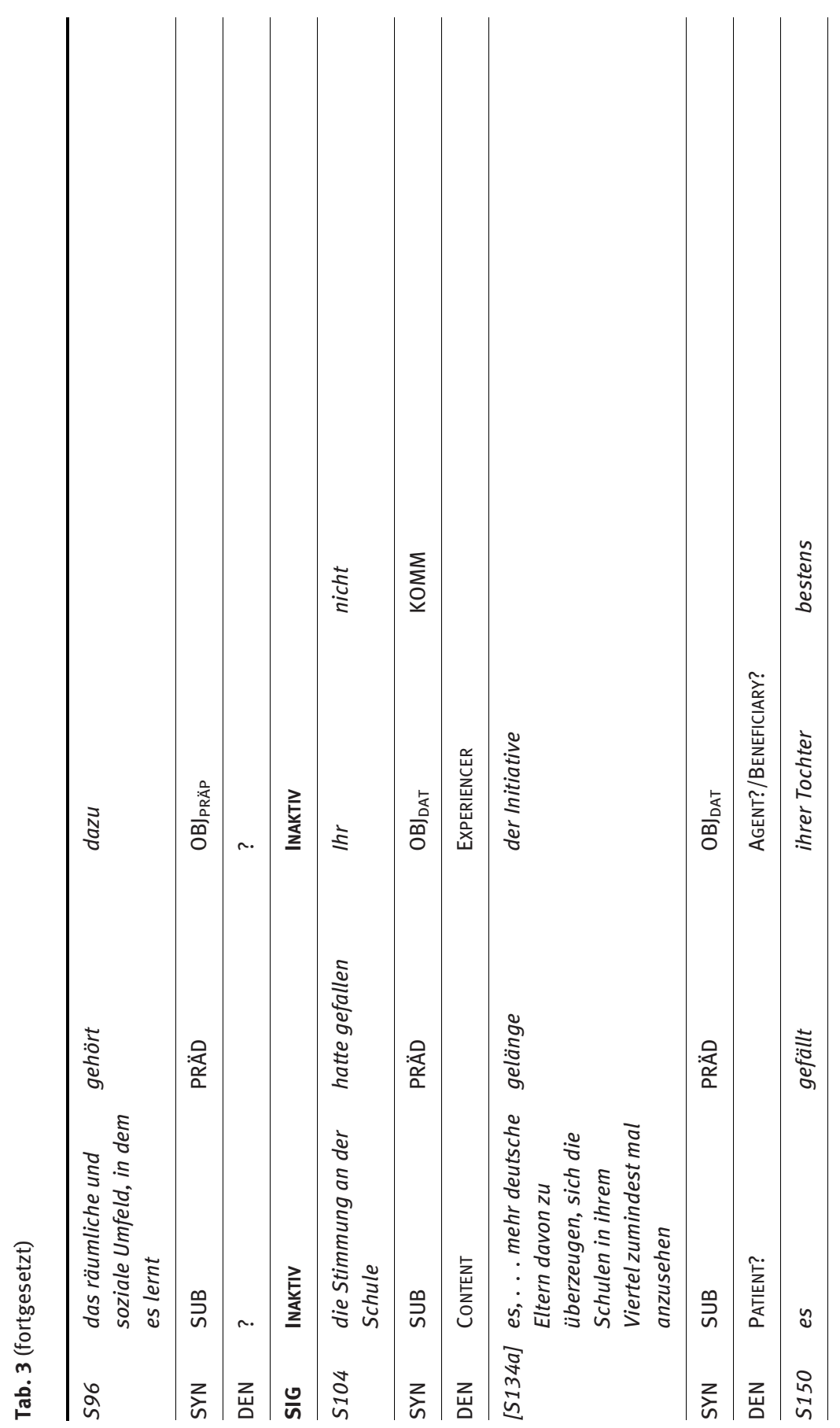




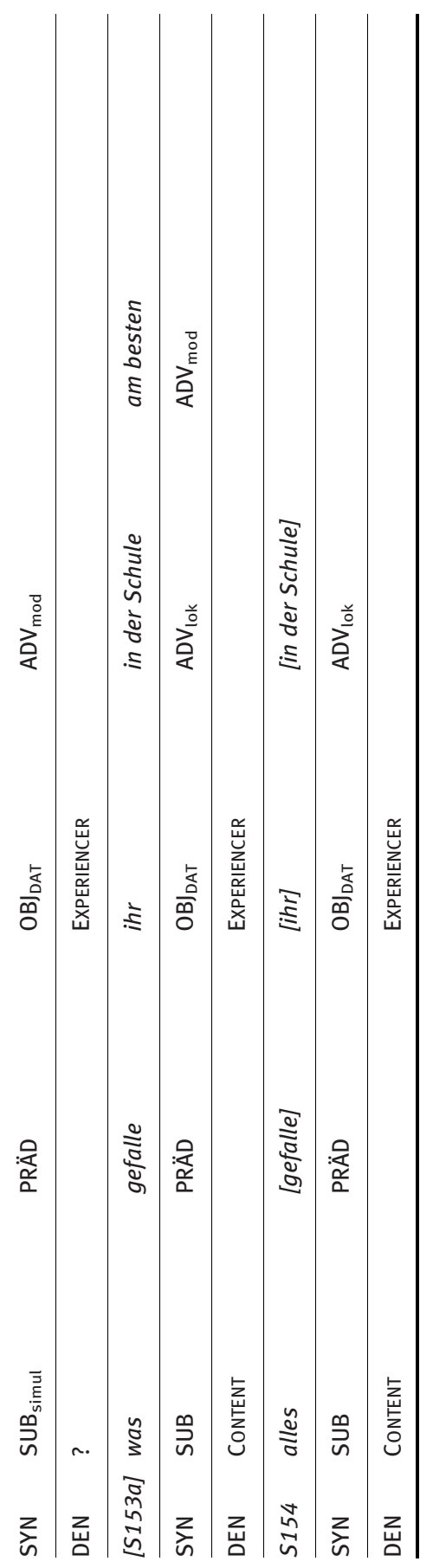


Soweit die theorievergleichende Textanalyse. Diese lässt einerseits konkrete Schlüsse auf die Probleme der denotativen Semantik zu und zeigt, wie sich diese signifikativ-semantisch lösen lassen (Kap. 5.1). Andererseits stellen die jeweiligen Zeilen SYN und SIG gegenwartsdeutsche Satzbauplan(zeichen)realisierungen auf signifikativ-semantischer Grundlage dar. Deren Systematisierung führt zur Modellierung der gegenwartsdeutschen Satzbaupläne (Kap. 5.2).

\section{Ergebnisse}

\subsection{Schlüsse aus der vergleichenden Textanalyse}

Das Verfahren im vorliegenden Kapitel ist das folgende: Zuerst gehen wir auf die Probleme bei der Anwendung des denotativ-semantischen Rollensets auf den Zeitungstext ein. Anschließend wird erläutert, wie sich diese Probleme signifikativ-semantisch lösen lassen.

Die Anwendung des denotativ-semantischen Rollensets ausgehend von den Fillmore'schen Begriffsbestimmungen war alles andere als einfach. Dies lag zum einen daran, dass für bestimmte Phänomentypen keine Rollen vorgesehen sind, zum anderen daran, dass die Zuordnung der Rollen unklar ist:

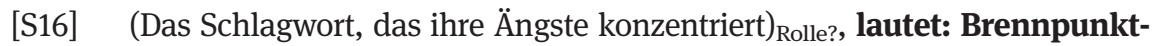
schule.

[S16a] Das [Schlagwort] konzentriert (ihre Ängste) Rolle? $_{\text {Ro }}$

[S2] Eine Initiative versucht (dagegen anzukämpfen) Rolle?

[S85h] sie kennen (es) Rolle? nicht anders aus ihrer Familie

[S2a] Eine Initiative kämpft (dagegen) $)_{\text {Rolle? }}$ an

[S85d] niemand kümmert sich (um ihre Hausaufgaben) Rolle? zu Hause

[S6] (Es) Rolle? war Ylvas erster Schultag

[S123a] Schaff $(\mathbf{t})_{\text {Rolle? }}$ mehr gute Angebote

[S86] Der klassische Konflikt ist (perfekt) Rolle?

[S101] Ich glaube (es ist sinnvoller, als Erwachsener in die Politik zu gehen, als das Kind zu benutzen, um Schulen stärker zu durchmischen) Rolle?

1) Generell fällt uns auf, dass die denotativ-semantische Analyse umso problematischer wird, je höher die sprachliche Abstraktheit der zu analysierenden Größen bzw. Sachverhalte ist: Z. B. sind das Subjekt in [S16] und das Akkusativobjekt in [S16a] so abstrakt, dass keine Rolle zuordenbar ist. Weder die Rollendefinitionen noch die sehr konkreten Beispielsätze helfen bei der Analyse. 
2) U. W. werden Subjekt- bzw. Objektnebensätzen oder Subjekt- bzw. Objektsinfinitivkonstruktionen wie in [S2] denotativ-semantisch keine semantischen Rollen zugewiesen. Das Gleiche gilt für propositionale es-Verwendungen wie in [S85h]. Die entsprechenden Rubriken in der Analyse mussten deshalb leer bleiben.

3) Da es denotativ-semantisch keine Präpositionalobjektsrollen gibt, haben wir beim Typus [S2a] versuchsweise GoAL eingetragen. Bei anderen Präpositionalobjekttypen wie in [S85d] musste die Präpositionalobjekt-Rubrik leer bleiben.

4) In denotativ-semantischen Arbeiten haben wir keine Hinweise darauf gefunden, wie man sog. formale Subjekte wie in [S6] (oder auch formale Objekte) interpretieren soll.

5) Bei Imperativsätzen wie [S123a], die Handlungssätze sind und deren Akkusativobjekt denotativ-semantisch PATIENT ist, ist uns unklar, ob sich AGENT irgendwo eintragen ließe oder ob Fillmore von Subjektlosigkeit ausgehen würde. Letztere Lösung würde implizieren, dass Handlungssätze nicht unbedingt Handelnde (AGENT) brauchen.

6) Eine weitere Schwäche der denotativ-semantischen Analyse wird durch unsere grammatische Analyse verdeckt: Entsprechend der Prädikatsauffassung der GTA (2017: 358-394) bilden die Prädikative zusammen mit den Kopulaverben das Prädikat (siehe auch die Belege (20) und (21) in Kap. 4.1). Denotative Semantikerinnen und Semantiker würden jedoch vermutlich eine formalgrammatische Analyse mit Prädikativen als eigenen Satzgliedern bevorzugen. Daraus folgt, dass denotativ-semantisch Prädikative eigene semantische Rollen bekommen müssten. Was das für Rollen sein könnten, ist uns nicht klar. Besonders problematisch dürfte die Rollenzuweisung bei adjektivischen Prädikativen wie in [S86] sein, die weder Gegenstände noch Sachverhalte indizieren.

7) Im Falle von grammatisch unterspezifizierten Objekten wie in [S101] kann denotativ-semantisch keine Rolle zugewiesen werden.

Was die obigen denotativ-semantischen Problemtypen anbelangt, lassen sie sich signifikativ-semantisch wie folgt lösen:

[S16] (Das Schlagwort, das ihre Ängste konzentriert) ZUSTANDSTRÄGER, lautet: $_{\text {a }}$ Brennpunktschule.

[S16a] Das [Schlagwort] konzentriert (ihre Ängste) HANDLungSGEGENSTAND

[S2] Eine Initiative versucht (dagegen anzukämpfen) HANDLUNGSSACHVERHALT $_{\text {fach }}$ 


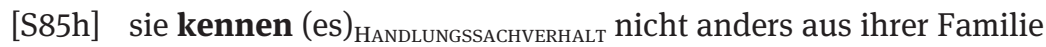

[S2a] Eine Initiative kämpft (dagegen) OPPonentum $_{\text {an }}$

[S85d] niemand kümmert sich (um ihre Hausaufgaben) АсcIPIEndum zu Hause

[S6] (Es) ZuSTANDSTRÄGERSIMULATION $_{\text {war Ylvas erster Schultag }}$

[S123a] Schaff $(\mathbf{t})_{\text {HandLUnGSTRÄGER }}$ mehr gute Angebote

[S86] Der klassische Konflikt (ist perfekt) Zustand

[S101] Ich glaube (es ist sinnvoller, als Erwachsener in die Politik zu gehen, als das Kind zu benutzen, um Schulen stärker zu durchmischen)HandLungs-/ TÄTIGKEITSSACHVERHALT

1) Was [S16] und [S16a] anbelangt, ergeben sich die signifikativ-semantischen Rollen aus der Kombination der jeweiligen Prädikatsrolle (ZuSTAND bzw. HANDLUNG) und der grammatischen Realisierung des jeweiligen Satzglieds (Subjekt bzw. Akkusativobjekt).

2) Signifikativ-semantisch bekommen Subjekt- bzw. Objektnebensätze, Subjektbzw. Objektsinfinitivkonstruktionen bzw. propositionale es-Verwendungen eine eigene Rolle, die sich aus der Prädikatsrolle und dem Sachverhaltscharakter der grammatischen Realisierung ergibt. In [S2] und [S85h] ergibt sich demnach aus HANDLUNG + SACHVERHALT die Rolle HANDLUNGSSACHVERHALT.

3) Signifikativ-semantisch haben alle Präpositionalobjekte eine eigene Rolle. Bei [S2a] ist diese OpPonENTUM, bei [S85d] AcCIPIENDUM. Im Unterschied zu denotativ-semantischen Rollen sind die signifikativen Rollen für genau eine Präpositionalobjektspräposition reserviert (Ausnahme ist einzig der Nischentransit (siehe Höllein 2019: 82-89)). Signifikativ-semantisch können die spezifischen Inhalte von Präpositionalobjekts-Präpositionen erfasst werden - im Falle der Präpositionalobjekts-Rolle ACCIPIENDUM z. B. das abstrakte Erlangenwollen. Weil denotativ-semantische Rollen für Präpositionalobjekte fehlen, müssen in der denotativen Semantik Rollen, die für andere Satzglieder entworfen worden sind, hilfsweise auf Präpositionalobjekte übertragen werden. Dadurch entsteht einerseits funktionale Synonymie, da dieselbe Rolle für verschiedene Satzglieder verwendet werden muss, andererseits ist die inhaltliche Erfassung unpräzise, da unterschiedliche Funktionen mit derselben Rolle beschrieben werden müssen.

4) Sog. formale Subjekte (wie auch formale Objekte) stellen Valenzsimulationen dar (Âgel 2000: 229, Anm. 27): „Valenzsimulationen sind [. . .] als Nachahmungen von Valenzrealisierungsstrukturen ohne realisierte Valenz(potenz) zu definieren.“ Das Es in [S6] simuliert also grammatisch das Subjekt. Signifikativ-semantisch ist es eine ZUSTANDSTRÄGERSIMULATION. 
5) In Imperativsätzen wie [S123a] ist das Subjekt mikrovalenziell realisiert, also ein Mikrosubjekt. Es fehlt also nicht, sondern ist in die Verbform inkorporiert. $^{32}$ Die Verbform vereint also zwei Satzgliedwerte: Prädikat und Subjekt. Signifikativ-semantisch ist das Mikrosubjekt in [S123a] HANDLUNGSTRÄGER. Dieser wird ja auch vom HANDLUNGSGEGENSTAND, der grammatisch Akkusativobjekt ist, vorausgesetzt.

6) Da Prädikative zusammen mit den Kopulaverben das Prädikat bilden, haben sie keine eigene semantische Rolle. Die Kopula ist und das prädikative Adjektiv perfekt tragen in [S86] gemeinsam die Prädikatsrolle ZUSTAND.

7) Bei grammatisch unterspezifizierten Objekten wie in [S101] lässt sich signifikativ-semantisch die grammatische Offenheit der Sachverhaltsdarstellung ikonisch abbilden: Da im Falle von glauben die Interpretation HANDLUNG etw. glauben - oder TÄTIGKEIT - an etw. glauben - offen bleibt, bleiben auch die Rollen offen: HANDLUNGS-/TÄTIGKEITSSACHVERHALT.

\subsection{Satzbaupläne des Gegenwartsdeutschen}

Wie in Kap. 4.3 erwähnt, stellen die jeweiligen Zeilen SYN und SIG der vergleichenden Textanalyse gegenwartsdeutsche Satzbauplan(zeichen)realisierungen auf signifikativ-semantischer Grundlage dar. Um von den Satzbauplanrealisierungen zum System der Satzbaupläne zu kommen und dabei von einem bekannten Archimedischen Punkt auszugehen, haben wir uns bei der nachfolgenden Modellierung der gegenwartsdeutschen Satzbaupläne am Duden (2016: 927-951) orientiert. Eine hundertprozentige Übereinstimmung gibt es trotzdem aus vier Gründen nicht:

1) Wie erwähnt, bilden entsprechend der Prädikatsauffassung der GTA 2017 die Prädikative zusammen mit den Kopulaverben das Prädikat. Dies gilt sowohl für Subjekts- wie auch für Objektsprädikative. Daraus folgt, dass es nach unserer Auffassung keine Subjekts- und Objektsprädikative als eigene Satzglieder gibt. Da die Freien Prädikative keine Komplemente sind, enthalten unsere Satzbaupläne überhaupt keine prädikativen Satzglieder.

32 Zur strukturellen Valenzrealisierung im Allgemeinen und zum Mikrosubjekt im Besonderen siehe Ágel (2000: 215-236). 
2) Nach Höllein (2019) werden zeichenhafte von nichtzeichenhaften Satzbauplänen unterschieden. Während Satzbauplanzeichen, die in der deutlichen Überzahl sind, nur aktive semantische Rollen enthalten, haben nichtzeichenhafte Satzbaupläne mindestens eine inaktive Rolle (siehe Kap. 3). Unsere Klassifikation der Satzbaupläne ist also zweigeteilt: eine umfangreiche Tabelle für den Prototyp, d. h. für die Satzbauplanzeichen, und eine kurze Tabelle für die Peripherie, $d$. $h$. für die nichtzeichenhaften Satzbaupläne.

3) Der Begriff ,Präpositionalobjekt liegt auf derselben begriffslogischen Ebene wie ,Kasusobjekt'. Folglich gibt es genauso wenig ein Satzglied ,Präpositionalobjekt', wie es ein Satzglied ,Kasusobjekt' gibt (GTA 2017: 282-283). Den Kasusobjekt-Satzgliedbegriffen ,Akkusativobjekt', ,Dativobjekt ${ }^{\bullet}$ und ,Genitivobjekt entsprechen 17 verschiedene Präpositionalobjekt-Satzgliedbegriffe wie z.B. ,Präpositional ${ }_{\mathrm{an}+\mathrm{DAT} \text {-objekt', ,Präpositional }}{ }_{\text {in }+A K K}$-objekt ${ }^{\bullet}$

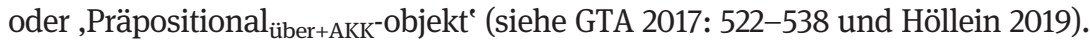
Deshalb wurden unsere Satzbaupläne dort, wo im Duden nur ,Präpositionalobjekt' steht, je nach Art des Präpositionalobjekts und der signifikativ-semantischen Rolle weiter differenziert.

4) Signifikativ-semantisch bilden Passivsätze eigene Satzbaupläne mit eigenen semantischen Rollen (Prädikatsklasse: VoRGANG, siehe z. B. Beleg [S135]).

Aus den genannten vier Gründen stimmen also unsere Satzbauplannummern nicht mehr mit denen im Duden 2016 überein. Wir haben aber - wo es möglich war - die Duden-Beispiele übernommen. Insofern besteht die Möglichkeit, die beiden Modelle - am besten ausgehend von den Beispielsätzen - zu vergleichen. 


\section{Satzbauplanzeichen}

\begin{tabular}{|c|c|c|}
\hline & Satzbauplan & Beispiele \\
\hline \multicolumn{3}{|c|}{ Prädikat ohne Ergänzung und semantische Rolle } \\
\hline 1 & Prädikat (VORGANG) & Auf dem Dach wird gearbeitet. \\
\hline \multicolumn{3}{|c|}{ Prädikat mit einer Ergänzung und einer semantischen Rolle } \\
\hline \multirow[t]{5}{*}{2} & [Subjekt] + Prädikat & \\
\hline & TÄTIGKEITSTRÄGER & [Sie] lacht/steht/stirbt/isst. \\
\hline & VORGANGSTRÄGER & $\begin{array}{l}\text { [Die Tür] öffnet sich/wird geöffnet./[Er] } \\
\text { wird Lehrer. }\end{array}$ \\
\hline & VORGANGSTRÄGERSIMULATION & [Es] regnet. \\
\hline & ZUSTANDSTRÄGER & $\begin{array}{l}\text { [Anna] ist zornig./[Anna] ist Lehrerin./[Der } \\
\text { Graustieltäubling] gilt als guter Speisepilz. }\end{array}$ \\
\hline \multirow[t]{2}{*}{3} & [Dativobjekt] + Prädikat & \\
\hline & ZUSTANDSBETROFFENER & [Mir] ist kalt/schwindlig/langweilig. \\
\hline
\end{tabular}

Prädikat mit zwei Ergänzungen und einer semantischen Rolle (weil eine Ergänzung = DIR/ ADV)

4 [Subjekt $]+[$ Direktivum $]+$ Prädikat

TÄTIGKEITSTRÄGER

[Sie] fährt [nach Hause]./[Die Gäste]

kommen [aus aller Herren Ländern]./[Sie]

zogen [durch die Wüste].

5 [Subjekt $]+[$ Lokaladverbial $]+$ Prädikat

TÄTIGKEITSTRÄGER

[Otto] wohnt [in Köln] .

ZUSTANDSTRÄGER

[Otto] ist [in Köln] wohnhaft.

6 [Subjekt $]+[$ Temporaladverbial $]+$ Prädikat

VORGANGSTRÄGER [Die Versammlung] währte [bis

Mitternacht].

7 [Subjekt $]+[$ Modaladverbial $]+$ Prädikat TÄTIGKEITSTRÄGER

[Die beiden Hunde] benahmen sich

[schlecht].

ZUSTANDSTRÄGER

[Die Luft] war [dreißig Grad] warm.

8 [Subjekt] $+[$ Kausaladverbial] + Prädikat

VORGANGSTRÄGER

[Der Mord] geschah [aus Eifersucht]. 
(fortgesetzt)

\section{Prädikat mit zwei Ergänzungen und zwei semantischen Rollen}

9 [Subjekt $]+[$ Akkusativobjekt $]+$ Prädikat HANDLUNGSTRÄGER - HANDLUNGSGEGENSTAND

HANDLUNSGTRÄGERSIMULATION HANDLUNGSGEGENSTAND
[Wir] bauen/besitzen [ein Haus]./[Er] isst [den Teller] leer./[Klaus] findet [Peter] klug./[Der Polizist] hielt [den Verhafteten] für den gesuchten Mörder./[Die Stadt] betrachtet [die gute Verkehrslage] als Standortvorteil./[Die Polizei] behandelte [die Demonstranten] wie Verbrecher. [Es] gibt [gute und schlechte Bücher] .

10 [Subjekt $]+[$ Dativobjekt $]+$ Prädikat HANDLUNGSTRÄGER - HANDLUNGSBETROFFENER

[Die Freunde] reden [mir] zu. ZUSTANDSTRÄGER - ZUSTANDSBETROFFENER

[Dieses Buch] gehört [mir]./[Dieser Termin] ist [mir] angenehm./[Mir] fehlt [das richtige Verständnis].

11 [Subjekt] + [Präpositionalobjekt] + Prädikat TÄTIGKEITS-/VORGANGS-/ZUSTANDSTRÄGER [jeweilige Präpositionalobjektsrolle 11a - 11y]

11a Präpositional ${ }_{a n+A K K}$-objekt/ADRESSATUM [Er] denkt [an seinen Arbeitskollegen]. ,Zielgröße der Übergabe“

11b Präpositional ${ }_{a n+D A T}$-objekt/AFFIZIERT [Der Hund] nagt [an einem Knochen]. ,partiell Erfasstes“

11c Präpositional ${ }_{\text {auf }+ \text { AKK-objekt/PROSPECTUM }}$ [Sie] wartet [auf die Blumen]. ,zukünftiges Ereignis‘

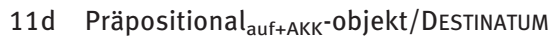

[Er] achtet [auf die Kinder]. ,Fokuspunkt der Aufmerksamkeit`

11e Präpositional ${ }_{\text {auf+AKK-objekt/BASIS }}$

[Die Firma] baut [auf ihre Mitarbeiter]. ,Grundlage des Szenarios‘

$11 \mathrm{f}$ Präpositional ${ }_{\text {auf+DAT}}$-objekt/BASIS ,Grundlage des Szenarios‘

[Das Geheimnis] lastet [auf der jungen Frau].

$11 \mathrm{~g}$ Präpositional ${ }_{\text {aus+DAT-objekt/MATERIAL }}$ ,Grundstoff‘

[Messing] besteht [aus Kupfer und Zink].

11h Präpositional für+AKK-objekt/BENEFACTUM $_{\text {- }}$ ,Nutznießer

[Die Kinder] sammeln [für die Dürreopfer]. 
(fortgesetzt)

11i Präpositional gegen+AKK-objekt/OPPONENTUM ,Gegengröße/Antipode‘

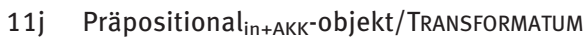
,Ergebnis einer erfolgten Umwandlung/ Metamorphose،
[Er] wendet sich [gegen die Bundesregierung].

[Die Sporthalle] verwandelt sich [in einen Indoorspielplatz].

11k Präpositional ${ }_{\text {in+DAT-objekt/DISZIPLIN }}$ [Er] versucht sich [im Schreiben]. ,abstrakter, im weiteren Sinn der Entwicklung zugehöriger Betätigungsraum‘

11l Präpositional mit+DAT-objekt/IDENTIFICATUM ,hat die Eigenschaft

[Mit Kroos] haben [sie] [einen herausragendenSpieler].

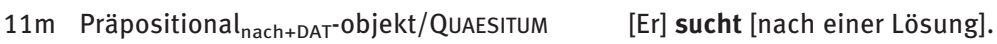
,das Gesuchte،

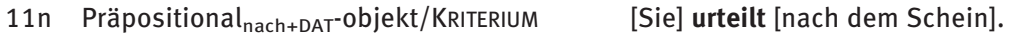
,Maßgröße‘

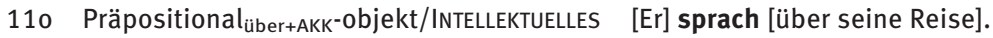
THEMA ,inhaltlicher Gegenstand der intellektuellen Aktivität‘

$11 \mathrm{p}$ Präpositional über+AKK-objekt/INFERIOR [Der König] herrscht [über seine ,ein hierarchisch Niedrigeres“ Untertanen].

11q Präpositional über+DAT-objekt/OKKUPATION $_{\text {- }}$ [Sie] grübelt [über dem Problem]. ,intensive, einnehmende und leicht negativ konnotierte Aktivität‘

11r Präpositional ${ }_{\text {um+AKK-objekt/ACCIPIENDUM }}$ [Sie] kämpfen [um den Comedy Pokal]. ,ein zu Erhaltendes“

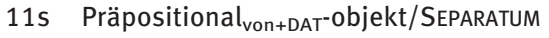

[Operettenhaus] trennt sich [von Gott]. ,das abstrakt Entnommene

11t Präpositional ${ }_{\text {von+DAT-objekt/PunKTUELles }}$ [Er] faselt [von Liebe]. THEMA ,inhaltlicher Gegenstand der oberflächlichen Aktivität‘

11u Präpositional ${ }_{\text {von+DAT-objekt/AUSLÖSER }}$ ,Initiator des Vorgangs“ 
(fortgesetzt)

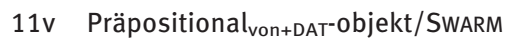

[Der Garten] wimmelt [von Bienen]. ,raumfüllende Entität‘

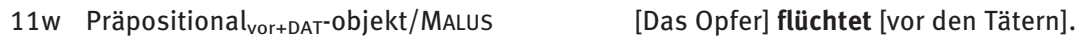
,negativ attribuierte Entität‘

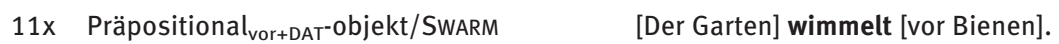
,raumfüllende Entität‘

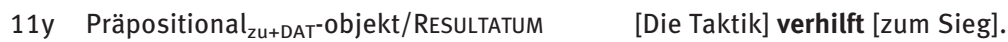
,Ergebnis‘

Prädikat mit drei Ergänzungen und einer semantischen Rolle (weil zwei Ergänzungen = ADV)

12 [Subjekt $]+[$ Lokaladverbial $]+$

[Modaladverbial] + Prädikat

VORGANGSTRÄGERSIMULATION

[Bei dem Fest] ging [es] [harmonisch] zu.

Prädikat mit drei Ergänzungen und zwei semantischen Rollen (weil eine Ergänzung = DIR/ADV)

13 [Subjekt $]+[$ Akkusativobjekt $]+$

[Direktivum] + Prädikat

HANDLUNGSTRÄGER - HANDLUNGSGEGENSTAND [Ich] hänge [das Bild] [an die Wand].

14 [Subjekt $]+[$ Dativobjekt $]+[$ Direktivum $]+$

Prädikat

TÄTIGKEITSTRÄGER - TÄTIGKEITSBETROFFENER [Ich] klopfe [ihm] [auf die Schulter].

15 [Subjekt $]+[$ Dativobjekt $]+$

[Modaladverbial] + Prädikat

ZUSTANDSTRÄGER - ZUSTANDSBETROFFENER

[Das Kleid] steht [seiner Lebensgefährtin] [gut].

$16^{33}$ [Subjekt $]+[$ Komitativadverbial $]+$

[Präpositionalobjekt] + Prädikat

TÄTIGKEITSTRÄGER - ACCIPIENDUM

[Die Spieler] wetten [mit dem Trainer] [um eine Flasche Sekt].

17 [Subjekt $]+[$ Präpositionalobjekt $]+$

[Modaladverbial] + Prädikat

TÄTIGKEITSTRÄGER - AFFIZIERT

[Er] handelte [niederträchtig] [an ihm].

33 Im Duden wird der Satzbauplan [Subjekt] + [Präpositionalobjekt] + [Präpositionalobjekt] angenommen. Wir analysieren die Präpositionalgruppe mit dem Trainer als Komitativadverbial. 
(fortgesetzt)

\section{Prädikat mit drei Ergänzungen und drei semantischen Rollen}

18 [Subjekt $]+[$ Akkusativobjekt $]+$ [Akkusativobjekt] + Prädikat HANDLUNGSTRÄGER - HANDLUNGSGEGENSTAND HANDLUNGSSACHVERHALT [Ich] habe [dich] [etwas/wann du kommst] gefragt!

19 [Subjekt $]+[$ Dativobjekt $]+$ [Akkusativobjekt] + Prädikat HANDLUNGSTRÄGER - HANDLUNGSBETROFFENER - $\quad$ [Der Junge] schenkte [seiner Mutter] HANDLUNGSGEGENSTAND [Rosen]./[Der Friseur] färbt [der Kundin] [die Haare] blond.

20 [Subjekt $]+[$ Dativobjekt $]+$ [Präpositionalobjekt] + Prädikat TÄTIGKEITSTRÄGER - TÄTIGKEITSBETROFFENER [Ich] verhelfe [dem Studenten] [zu einer RESULTATUM Stellung].

21 [Subjekt $]+[$ Akkusativobjekt $]+$ [Präpositionalobjekt] + Prädikat HANDLUNGSTRÄGER - HANDLUngSGEGENSTAND - $\quad$ [Der Intendant] bat [die Zuschauer] [um [jeweilige Präpositionalobjektsrolle 21a- Geduld]. 21y]

21a Präpositional ${ }_{a n+A K K}$-objekt/ADRESSATUM ,Zielgröße der Übergabe‘

[Er] schreibt [einen Brief] [an seinen Arbeitskollegen].

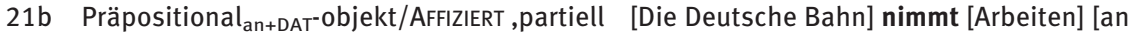
Erfasstes، Gleisen] vor.

21c Präpositional ${ }_{\text {an+DAT}}$-objekt/DETECTUM ,Maßgröße der Klassifikation“

[Major-Labels] messen [den Erfolg] [an Verkaufszahlen].

21d Präpositional auf+AKK-objekt/PROSPECTUM ,zukünftiges Ereignis‘

[Sie] bereitet [ihn] [auf kommende Einsätze] vor.

21e Präpositional ${ }_{\text {auf+AKK-objekt/DESTINATUM }}$ [Anna] sprach [ihn] [auf ihr Auto] an. ,Fokuspunkt der Aufmerksamkeit

$21 \mathrm{f}$ Präpositional ${ }_{\text {auf+AKK-objekt/BASIS }}$ ,Grundlage des Szenarios‘

[Die Firma] stützt [ihre Planung] [auf Prognosen].

$21 \mathrm{~g}$ Präpositional ${ }_{\text {auf+DAT-objekt/BASIS }}$ [Die Forscher] begründen [ihre Theorie] ,Grundlage des Szenarios‘ [auf einfachen Überlegungen]. 
(fortgesetzt)

21h Präpositional ${ }_{\text {aus+DAT-objekt/MATERIAL }}$ ,Grundstoff‘

21i Präpositional für+AKK-objekt/BENEFACTUM $_{\text {- }}$ ,Nutznießer

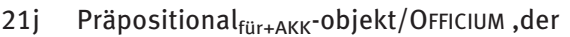
Gegenleistung zugrundeliegende Leistung، Verhalten].

21k Präpositional ${ }_{\text {gegen+AKK-objekt/OPPONENTUM }}$ ,Gegengröße/Antipode‘

21l Präpositional ${ }_{\text {in+AKK-objekt/TRANSFORMATUM }}$ ,Ergebnis einer erfolgten Umwandlung/ Metamorphose“
[Er] bastelt [ein Flugzeug] [aus Pappe].

[Wir] nutzen [unsere Fehler] [für unsere Entwicklung].

[Er] bestraft [die Kinder] [für ihr

[Der Despot] setzt [Panzer] [gegen die Bevölkerung] ein.

[Er] wandelt [die $\mathrm{GmbH}$ ] [in eine Aktiengesellschaft] um.

[Sie] prüft [die Schüler] [in Mathe].

$21 \mathrm{~m}$ Präpositional ${ }_{\text {in+DAT-objekt/DISZIPLIN }}$ ,abstrakter, im weiteren Sinn der Entwicklung zugehöriger Betätigungsraum ‘

21n Präpositional ${ }_{\text {in+DAT-objekt/RESPONS , das }}$ Erkannte“

[Er] erkennt [seinen Bruder] [in dem Fremden].

210 Präpositional mit+DAT-objekt/IDENTIFICATUM ,hat die Eigenschaft"

[Mit seiner neuen Platte] präsentierte [Westernhagen] [ein Meisterwerk].

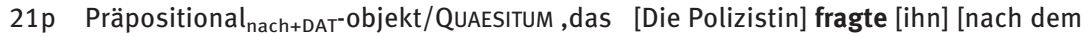
Gesuchte* Ausweis].

21q Präpositional nach+DAT-objekt/KRITERIUM ,Maßgröße“

[Sportjournalisten] beurteilen [Stürmer] [nach Toren].

21r Präpositional über+AKK-objekt/INTELLEKTUELLES THEMA ,inhaltlicher Gegenstand der

[Der Arzt] informiert [die Eltern] [über ihre intellektuellen Aktivität‘

21s Präpositional über+DAT-objekt/OKKUPATION $_{\text {- }}$ ,intensive, einnehmende und leicht negativ [Sie] vergisst [die Arbeit] [über dem konnotierte Aktivität‘ Verletzung].

Gespräch].

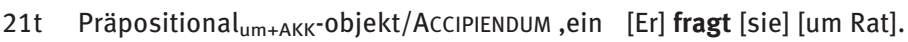
zu Erhaltendes“

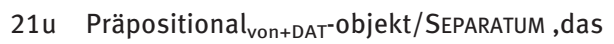
abstrakt Entnommene،

[Die Kontrolleure] entlasteten [den Minister] [von den Vorwürfen]. 
(fortgesetzt)

21v Präpositional von+DAT-objekt/ $_{\text {- }}$

Punktuelles Thema, inhaltlicher

Gegenstand der oberflächlichen

Aktivität ${ }^{*}$

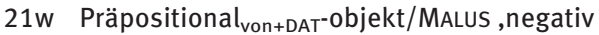
attribuierte Entität

21x Präpositional ${ }_{\text {vor+DAT-objekt/SWARM }}$ ,raumfüllende Entität‘

21y Präpositional ${ }_{\text {zu+DAT}}$-objekt/RESULTATUM ,Ergebnis‘
[Sie] informieren [ihre

Krankenversicherung] [von ihrem

Auslandsaufenthalt].

[Deiche] schützen [die Bürger] [vor den Fluten].

[Er] jagt [sie] [vor Eifersucht].

[Sie] ordnen [die Eindrücke] [zu Mustern].

\section{Prädikat mit vier Ergänzungen und drei semantischen Rollen}

22 [Subjekt] + [Dativobjekt $]+$

[Akkusativobjekt] + [Direktivum] +

Prädikat

HANDLUNGSTRÄGER - HANDLUNGSBETROFFENER

- HANDLUNGSGEGENSTAND

[Er] legt [ihm] [die Hand] [auf die Schulter].

\section{Nichtzeichenhafte Satzbaupläne}

1 Satzbauplan

Beispiele

\section{Prädikat mit einer Ergänzung}

2 [Akkusativobjekt] + Prädikat

[Mich] hungert.

\section{Prädikat mit zwei Ergänzungen}

3 [Subjekt] $+[$ Akkusativobjekt $]+$ Prädikat

[Sie] ist [den Lärm] gewohnt.

4 [Subjekt $]+[$ Genitivobjekt $]+$ Prädikat

[Wir] harrten [der Dinge].

[Er] ist [des Diebstahls] schuldig.

5 [Subjekt $]+[$ Präpositionalobjekt $]+$

[Sie] verzichtet [auf das Geschenk]. Prädikat

6 [Akkusativobjekt]/[Dativobjekt] +

[Mich]/[Mir] ekelt [vor diesem Essen]. [Präpositionalobjekt] + Prädikat

7 [Subjekt] + [Akkusativobjekt] + [Genitivobjekt] + [Die Zeugen] beschuldigen [den Mann] Prädikat [des Diebstahls]. 


\section{Fazit}

Ziel der Studie war zunächst, das bislang existierende Satzbauplankonzept von mehr oder weniger rein ausdrucksseitigen Größen im Sinne der Saussure'schen Semiotik und konstruktionsgrammatischer Argumentstrukturmuster $\mathrm{zu}$ ausdrucks- und inhaltsseitigen Satzbauplanzeichen weiterzuentwickeln. Die so modellierten Strukturen werden hier als Satzbauplanzeichen gefasst. Die Inhaltsseiten der Satzbauplanzeichen werden als semantische Rollen modelliert, wobei sich die angewendete signifikative Semantiktheorie radikal von der Mainstreamtheorie, die als denotative Semantiktheorie bezeichnet wird, unterscheidet. Ein weiteres Ziel war es also, die signifikative Semantik zu präsentieren, bzw. die Leistungsfähigkeit eines vollständigen signifikativsemantischen Rollensets und dessen Überlegenheit gegenüber denotativen Konzeptionen nachzuweisen.

Dieser Nachweis wurde anhand einer Parallelanalyse geführt, bei der sich gezeigt hat, dass die Schwächen der denotativen Semantik insbesondere bei der Analyse abstrakter Strukturen enorm sind. D. h. immer dann, wenn Sachverhalte oder auch nur Satzglieder lexikalisch abstrakter gefüllt sind, wird eine denotativ-semantische Rollenanalyse problematisch, wie die - signifikativ-semantisch völlig unproblematische - Analyse von es-Strukturen illustriert hat. Während die denotativ-semantische Analyse von Kernsatzgliedern wie Subjekt und Akkusativobjekt hier bereits an Grenzen stößt, scheitert sie bei der Analyse von Präpositionalobjekten komplett, da zur semantischen Analyse von Präpositionalobjekten keine denotativ-semantischen Rollen bereitstehen. In Bezug auf die denotativ-semantische Rolle THEME ist aufgefallen, dass sich das verbreitete Verständnis der Rolle als ,Thema' nicht mit der von Fillmore intendierten Bedeutung deckt: „,an entity to which the verb’s meaning assigns a location or a change of location““ (Fillmore 2003: 464). Die Rolle steht damit pars pro toto für die denotative Rollensemantik: Bei Analysen müssen denotative Rollen immer wieder ad hoc an Gegebenheiten angepasst, interpretiert oder umdefiniert werden. Verfestigt sich diese Praktik, kann sie Rollenverständnisse so nachhaltig verändern, wie es bei THEME der Fall ist.

Die präsentierte Parallelanalyse verdeutlicht einerseits, dass die signifikative Semantik mehr als nur eine Alternative zu existierenden Ansätzen ist, da nur sie Texte umfänglich und lückenlos rollensemantisch analysieren kann. Andererseits empfehlen sich signifikativ-semantische Rollen zur Modellierung der Inhaltsseiten von Satzbauplanzeichen bzw. konstruktionsgrammatischer Argumentstrukturmuster, da Letztere noch immer denotativ-semantisch basiert sind. 
Es ist wichtig zu betonen, dass die von uns vorgelegte und empirisch erprobte Liste signifikativ-semantischer Rollen keine bloße Erweiterung bisheriger Listen darstellt, sondern das Ergebnis einer grundsätzlich anderen Herangehensweise ist, bei der Präzisierungen und Korrekturen durchaus angebracht und erwartbar sind, aber Beliebigkeit ausgeschlossen ist. Während nämlich Denotativität auf Bezeichnung basiert und somit wegen der Unendlichkeit der Welt nie abschließbar ist, basiert Signifikativität auf einzelsprachlichen Strukturen und Bedeutungen, die zwar Modelle sind, aber gewiss nicht beliebig erweitert und modifiziert werden können. ${ }^{34}$

\section{Literatur}

\section{Quellen}

Freud Moses = Freud, Sigmund (1914): Der Moses des Michelangelo. In Anna Freud (Hrsg.)

(1981), Sigmund Freud: Gesammelte Werke. Werke aus den Jahren 1913-1917. Bd. X, 172-

201. Frankfurt a. M: Fischer.

Haas Silentium = Haas, Wolf (1999): Silentium. Reinbek bei Hamburg: Rowohlt (rororo 23822).

Strittmatter Bienkopp = Strittmatter, Erwin (1963/2003): Ole Bienkopp. Roman. Leipzig: Faber \& Faber.

\section{Sekundärliteratur}

Admoni, Wladimir G. (1974): Die Satzmodelle und die logisch-grammatischen Typen des

Satzes. Deutsch als Fremdsprache 11, 34-42.

Admoni, Wladimir G. (1982): Der deutsche Sprachbau. München: Beck.

Ágel, Vilmos (2000): Valenztheorie. Tübingen: Narr.

Ágel, Vilmos (2015): Brisante Gegenstände. Zur valenztheoretischen Integrierbarkeit von Konstruktionen. In Stefan Engelberg, Meike Meliss, Kristel Proost \& Edeltraud Winkler (Hrsg.), Argumentstruktur zwischen Valenz und Konstruktionen. Empirie - Theorie Anwendung. Tagung an der Universität Santiago de Compostela 26.-28. September 2012, 61-87. Tübingen: Narr.

34 Unser Beitrag ist im Rahmen des DFG-Langfristprojekts Syntaktische Grundstrukturen des Neuhochdeutschen. Zur grammatischen Fundierung eines Referenzkorpus Neuhochdeutsch (https://gieskane.com) entstanden. In diesem Projekt wird das präsentierte System signifikativ-semantischer Rollen seit 2016 erfolgreich auf ein Korpus neuhochdeutscher Texte angewendet, indem alle grammatischen Sätze des Korpus auch semantisch annotiert werden. Wir danken den Bandherausgeberinnen und den anonymen GutachterInnen für Vorschläge und Anregungen sowie Maria Gallinat und Nina Reichenbach für ein sehr präzises und wertvolles Korrekturlesen. 
Ágel, Vilmos (2019): Grammatische Textanalyse (GTA) - eine deszendente Syntax des Deutschen. In Ludwig M. Eichinger \& Albrecht Plewnia (Hrsg.), Neues vom heutigen Deutsch. Empirisch - methodisch - theoretisch. Jahrbuch des Instituts für Deutsche Sprache 2018, 265-291. Berlin, Boston: De Gruyter.

Allwood, Jens, Lars-Gunnar Andersson \& Östen Dahl (1973): Logik für Linguisten. Tübingen: Niemeyer (= Romanistische Arbeitshefte 8).

Bossong, Georg (1992): Zum Begriff des Subjekts in Sprachtypologie und Universalienforschung. In Susanne R. Anschütz (Hrsg.), Texte, Sätze, Wörter und Moneme. Festschrift für Klaus Heger zum 65. Geburtstag, 105-122. Heidelberg: Orient.

Brinkmann, Hennig (1971): Die deutsche Sprache. Gestalt und Leistung. Düsseldorf: Schwann. Chomsky, Noam (1993): Lectures on government and binding. The Pisa Lectures. Berlin, New York: De Gruyter (= Studies in Generative Grammar).

Coseriu, Eugenio (1970): Bedeutung und Bezeichnung im Lichte der strukturellen Semantik. In Peter Hartmann \& Henri Vernay (Hrsg.), Sprachwissenschaft und Übersetzen. Symposion an der Universität Heidelberg. 24. 2.-26.2.1969, 1-18. München: Hueber.

Croft, William (2001): Radical construction grammar. Syntactic theory in typological perspective. Oxford: University Press.

Dowty, David R. (1991): Thematic proto-roles and argument selection. Language 67, 547-619. Duden 2016 = Wöllstein, Angelika \& die Dudenredaktion (Hrsg.), Duden. Die Grammatik. 9., vollständig überarbeitete und aktualisierte Aufl. Berlin: Dudenverlag (= Der Duden 4).

Engel, Ulrich (1970): Die deutschen Satzbaupläne. Wirkendes Wort 20, 361-392.

Engelen, Bernhard (1975/1-2): Untersuchungen zu Satzbauplan und Wortfeld in der geschriebenen deutschen Sprache der Gegenwart. 2 Teilbände. München: Hueber (= Heutiges Deutsch I/3.1-2).

Erben, Johannes (1980): Deutsche Grammatik. Ein Abriß. München: Hueber.

Feilke, Helmuth (1996): Sprache als soziale Gestalt. Ausdruck, Prägung und die Ordnung der sprachlichen Typik. Frankfurt a. M.: Suhrkamp.

Fillmore, Charles J. (1968): The case for case. In Emmon W. Bach, E. \& Robert T. Harms (Hrsg.), Universals in linguistic theory, 21-119. New York: Rinehart and Winston.

Fillmore, Charles J. (2003): Valency and semantic roles. The concept of deep structure case. In Vilmos Ágel, Ludwig M. Eichinger, Hans-Werner Eroms, Peter Hellwig, Hans Jürgen Heringer \& Henning Lobin (Hrsg.), Dependenz und Valenz. Ein internationales Handbuch der zeitgenössischen Forschung. Band 1, 457-475. Berlin, New York: De Gruyter.

Fillmore, Charles J. (2006): Frame semantics. In Keith Brown (Hrsg.), Encyclopedia of Language \& Linguistics, 613-620. Amsterdam: Elsevier.

Fischer, Klaus (2003): Verb, Aussage, Valenzdefinition und Valenzrealisierung: auf dem Weg zu einer typologisch adäquaten Valenztheorie. In Klaas Willems, Ann Coene \& Jeroen van Pottelberge (Hrsg.), Valenztheorie. Neuere Perspektiven, 14-64. Gent: Academia Press (= Studia Germanica Gandensia 2003.2).

Fleischmann, Eberhard (1985): Kasustheorie und Translationslinguistik. Dissertation zur Promotion B. Leipzig.

Goldberg, Adele (1995): Constructions: A construction grammar approach to argument structure. Chicago, London: University of Chicago Press.

Götze, Lutz (1979): Valenzstrukturen deutscher Verben und Adjektive. Eine didaktische Darstellung für das Fach Deutsch als Fremdsprache. München: Hueber (= Heutiges Deutsch III.3). 
GTA 2017 = Ágel, Vilmos (2017): Grammatische Textanalyse. Textglieder, Satzglieder, Wortgruppenglieder. Berlin, Boston: De Gruyter.

Haspelmath, Martin (1993): More on the typology of inchoative/causative alternations. In Bernard Comrie \& Maria Polinsky (Hrsg.), Causatives and transitivity, 87-120. Amsterdam, Philadelphia: John Benjamins (= Studies in Language Companion Series 23). Helbig, Gerhard (1979): Zum Status der Valenz und der semantischen Kasus. Deutsch als Fremdsprache 16, 65-78.

Helbig, Gerhard (1982): Valenz - Satzglieder - semantische Kasus - Satzmodelle. Leipzig: Enzyklopädie (= Zur Theorie und Praxis des Deutschunterrichts für Ausländer).

Helbig, Gerhard (1992): Probleme der Valenz- und Kasustheorie. Tübingen: Niemeyer (= Konzepte der Sprach- und Literaturwissenschaft 51).

Höllein, Dagobert (2017): Gibt es Handlungs-, Tätigkeits-, Vorgangs- und Zustandsverben? Ein Vorschlag für signifikativ-semantische Rollen von Prädikaten. Zeitschrift für germanistische Linguistik 45 (2), 286-305.

Höllein, Dagobert (2019): Präpositionalobjekt vs. Adverbial. Die semantischen Rollen der Präpositionalobjekte. Berlin, Boston: De Gruyter (= Linguistik - Impulse und Tendenzen 82).

Ickler, Irene (1990): Kasusrahmen und Perspektive. Zur Kodierung von semantischen Rollen. Deutsche Sprache 18, 1-37.

Köpcke, Klaus-Michael \& Christian Hinze (2011): Präpositionalobjekt und Präpositionaladverbial. Vom Nutzen der Prototypentheorie für den Grammatikunterricht. In Klaus-Michael Köpcke (Hrsg.), Sprachliche Strukturen thematisieren. Sprachunterricht in Zeiten der Bildungsstandards, 60-83. Baltmannsweiler: Schneider Hohengehren.

KVL 1978 = Engel, Ulrich \& Helmut Schumacher (1978): Kleines Valenzlexikon deutscher Verben. Tübingen: Narr (= Forschungsberichte des IdS 31).

Lasch, Alexander (2016): Nonagentive Konstruktionen des Deutschen. Berlin, Boston: De Gruyter (= Sprache und Wissen 25).

Lerot, Jacques (1982): Die verbregierten Präpositionen in Präpositionalobjekten. In Werner Abraham (Hrsg.), Satzglieder im Deutschen. Vorschläge zur syntaktischen, semantischen und pragmatischen Fundierung, 261-291. Tübingen: Narr.

Levin, Beth (1999): Objecthood. An event structure perspective. CLS 35 (1), 223-247.

Levin, Beth \& Malka Rappaport Hovav (2008): Argument realization. Cambridge: Cambridge University Press (= Research Surveys in Linguistics).

Löbner, Sebastian (2018): Barsalou-Frames in Wort- und Satzsemantik. In Stefan Engelberg, Hennig Lobin, Kathrin Steyer \& Sascha Wolfer (Hrsg.), Wortschätze. Dynamik, Muster, Komplexität, 189-212. Berlin, Boston: De Gruyter (= Jahrbuch des Instituts für Deutsche Sprache XXIV).

Müller, Wolfgang (2012): Wörterbuch deutscher Präpositionen. Die Verwendung als Anschluss an Verben, Substantive, Adjektive und Adverbien. Berlin, New York: De Gruyter.

Polenz von, Peter (2008): Deutsche Satzsemantik. Grundbegriffe des Zwischen-den-ZeilenLesens. 3. Aufl. Berlin, New York: De Gruyter (= De Gruyter Studienbuch).

Primus, Beatrice (1999): Cases and thematic roles. Ergative, accusative and active. Tübingen: Niemeyer (= Linguistische Arbeiten 393).

Primus, Beatrice (2004): Protorollen und Verbtyp. Kasusvariation bei psychischen Verben. In Rolf Kailuweit \& Martin Hummel (Hrsg.), Semantische Rollen, 377-401. Tübingen: Narr.

Primus, Beatrice (2012): Semantische Rollen. Heidelberg: Winter (= Kurze Einführungen in die germanistische Linguistik 12). 
Rostila, Jouni P. (2005): Zur Grammatikalisierung bei Präpositionalobjekten. In Torsten Leuschner, Tanja Mortelmans \& Sarah de Groodt (Hrsg.), Grammatikalisierung im Deutschen, 135-168. Berlin, New York: De Gruyter.

Rostila, Jouni P. (2007): Konstruktionsansätze zur Argumentmarkierung im Deutschen. Tampere: Tampere University Press.

Sauermann, Eberhard (1985): Zu Valenzverstößen in poetischer Sprache. Befremdende Transitivierungen bei Georg Trakl. In Erwin Koller \& Hans Moser (Hrsg.), Studien zur deutschen Grammatik. Johannes Erben zum 60. Geburtstag, 335-356. Innsbruck (= Innsbrucker Beiträge zur Kulturwissenschaft: Germanistische Reihe 25).

Saussure, Ferdinand de (2003): Wissenschaft der Sprache. Neue Texte aus dem Nachlass. Frankfurt a. M.: Suhrkamp (= Suhrkamp Taschenbuch Wissenschaft 1677).

Schneider, Jan Georg (2014): In welchem Sinne sind Konstruktionen Zeichen? Zum Begriff der Konstruktion aus semiologischer und medialitätstheoretischer Perspektive. In Alexander Lasch \& Alexander Ziem (Hrsg.), Grammatik als Inventar von Konstruktionen?, 357-374. Berlin, Boston: De Gruyter (= Sprache und Wissen 15).

Schumacher, Helmut (1975): Zum Problem der Satzmodelle. In Ulrich Engel \& Paul Grebe (Hrsg.), Sprachsystem und Sprachgebrauch. Festschrift für Hugo Moser, 360-372. Teil 2. Düsseldorf: Schwann (= Sprache der Gegenwart 34).

Starosta, Stanley (1978): The One per Sent Solution. In Werner Abraham (Hrsg.), Valence, semantic case, and grammatical relations, 439-576. Amsterdam: John Benjamins.

Starosta, Stanley (1988): The case for lexicase. An outline of lexicase grammatical theory. London, New York: Pinter (= Open Linguistics Series).

Tarvainen, Kalevi (1981): Einführung in die Dependenzgrammatik. Tübingen: Niemeyer (= Reihe Germanistische Linguistik 35).

Ullmann, Stephen (1973): Semantik. Eine Einführung in die Bedeutungslehre. Frankfurt a. M.: Fischer (= Conditio humana).

Vuillaume, Marcel (2003): Valenz und Satzbauplan. In Vilmos Ágel, Ludwig M. Eichinger, Hans-Werner Eroms, Peter Hellwig, Hans Jürgen Heringer \& Henning Lobin (Hrsg.), Dependenz und Valenz. Ein internationales Handbuch der zeitgenössischen Forschung. Band 1, 484-498. Berlin, New York: De Gruyter (= HSK 24.1).

Welke, Klaus (1988): Einführung in die Valenz- und Kasustheorie. Leipzig: Bibliographisches Institut.

Welke, Klaus (1994): Thematische Relationen. Sind thematische Relationen semantisch, syntaktisch oder/und pragmatisch zu definieren? In Deutsche Sprache 22, 1-18.

Welke, Klaus (1995): Dependenz, Valenz und Konstituenz. In Ludwig M. Eichinger \& Hans-Werner Eroms (Hrsg.), Dependenz und Valenz, 163-175. Hamburg: Buske (= Beiträge zur germanistischen Sprachwissenschaft 10).

Welke, Klaus (2005): Deutsche Syntax funktional. Perspektiviertheit syntaktischer Strukturen. 2. Aufl. Tübingen: Stauffenburg (= Stauffenburg Linguistik 22).

Welke, Klaus (2011): Valenzgrammatik des Deutschen. Eine Einführung. Berlin, New York: De Gruyter.

Welke, Klaus (2019): Konstruktionsgrammatik des Deutschen. Ein sprachgebrauchsbezogener Ansatz. Berlin, Boston: De Gruyter (= Linguistik - Impulse \& Tendenzen 77).

Ziem, Alexander \& Alexander Lasch (2013): Konstruktionsgrammatik. Konzepte und Grundlagen gebrauchsbasierter Ansätze. Berlin: De Gruyter (= Germanistische Arbeitshefte 44). 


\section{Anhang: der analysierte Text}

Vorsicht, Brennpunkt! Viele Eltern aus Berlin-Neukölln schulen ihre Kinder lieber in anderen Vierteln ein. Eine Initiative versucht nun, dagegen anzukämpfen

von Nicola Meier

DIE ZEIT № 35/2013, 30. August 2013 14:56 Uhr

Lange hatte sich Ylva Lafrenz auf diesen Moment gefreut. Mit ihrer Schultüte im Arm stand die Fünfjährige vor ein paar Tagen auf dem Schulhof der Karl-Weise-Schule in Berlin inmitten vieler unbekannter Kinder. Nur wenige waren so blond wie sie. Es war Ylvas erster Schultag.

Gerade mal fünf Minuten braucht die Erstklässlerin von ihrem Zuhause bis in die neue Schule. Viel näher kann man es in einer Großstadt wie Berlin nicht haben. Dass Ylva nun direkt um die Ecke eingeschult wurde, ist alles andere als selbstverständlich, denn sie wohnt in Neukölln. In der Karl-Weise-Schule lernen 285 Schüler, die meisten kommen aus türkischen und arabischen Familien. Insgesamt haben 80 Prozent der Kinder einen Migrationshintergrund, an anderen Grundschulen im Bezirk sind die Zahlen ähnlich hoch.

Spätestens seitdem im Jahr 2006 die Lehrer der Rütli-Schule öffentlich um Hilfe riefen und ihre Schule so deutschlandweit in die Schlagzeilen brachten, steht der Stadtteil Neukölln für ein Umfeld, in dem Eltern sich ihr Kind lieber nicht vorstellen möchten. Das Schlagwort, das ihre Ängste konzentriert, lautet: Brennpunktschule.

Nun stehen viele dieser Brennpunktschulen ausgerechnet dort, wo junge Eltern mittlerweile gerne wohnen. Dieses Dilemma gibt es in den meisten Großstädten, aber nirgendwo ist es so ausgeprägt wie in Berlin. Kreuzberg ist so ein Viertel, aber auch Neukölln, ein Stadtteil im Süden Berlins, so groß wie Bielefeld, als Problembezirk bekannt und lange als Ausländerhochburg und HartzIV-Ghetto verschrien. Dann zogen die niedrigen Mieten immer mehr Künstler und Studenten an, Galerien und Bars wurden eröffnet, und plötzlich war Neukölln ein hippes Multikulti-Viertel, in dem man gerne wohnt. Bis das erste Kind in die Schule kommt.

Lilia Kleemann, 33 Jahre alt, Designerin, entschied sich gegen die Schule vor der Haustür. Es ist viertel vor acht, als sie mit ihrem sechsjährigen Sohn Liou an der U-Bahn-Haltestelle ankommt, wo schon andere Mütter und Väter stehen. Großes Hallo, Umarmungen. Dann läuft Kleemann mit sechs Kindern zur Rolltreppe, die anderen Eltern eilen zurück zu ihren Autos und Fahrrädern. Sie wohnen in Kreuzberg und Neukölln, aber ihre Kinder gehen in Tempelhof auf eine Privatschule. Zwei U-Bahn-Stationen mit der U6, umsteigen, sieben Stationen mit der U7, noch mal fünf Minuten Fußweg. Gute 40 Minuten Schulweg, morgens 
hin, nachmittags zurück. Die Eltern haben eine Fahrgemeinschaft gegründet, jeder ist einmal die Woche mit Hinbringen und Abholen dran.

Es gebe Schulen in Berlin, in denen kein einziges deutsches Kind aus dem Einzugsgebiet komme, schimpft Neuköllns Bürgermeister Heinz Buschkowsky in seinem Buch Neukölln ist überall. Schulflucht nennt er es, wenn Eltern ihre Kinder außerhalb ihrer Wohnbezirke einschulen, und kritisiert das scharf. Wer gerne in einer günstigen Gründerzeitwohnung in Neukölln lebe, der möge doch bitte auch sein Kind dort in die Schule schicken. So wie Buschkowsky denkt auch Petra Lafrenz, die Mutter der Erstklässlerin Ylva, obwohl sie Buschkowskys Buch nie gelesen hat. „Eltern, die ihre Kinder außerhalb einschulen, verhindern die Integration“, sagt sie. Ihre Tochter Ylva geht deshalb nun in die Karl-Weise-Schule gleich um die Ecke. Zusammen mit anderen Eltern hat Lafrenz die Initiative „Kiezschule für alle“ gegründet, eine Art Gegenprogramm zur Schulflucht.

Lafrenz wohnt schon seit 30 Jahren in Neukölln, sie hat erlebt, wie Jugendgangs durch die Straßen zogen und auf Spielplätzen die Geräte brannten. Dort, wo sie wohnt, sieht Neukölln auch heute noch aus wie sein Klischee: Call-Shops neben Ein-Euro-Läden, auf den Straßen sind mehr Frauen mit Kopftüchern unterwegs als Hipster mit Röhrenjeans und Jutebeutel. Lafrenz, eine späte Mutter, ist Informatikerin, aber je länger man mit ihr redet, desto eher hält man sie für eine Sozialpädagogin, gutgläubig und immer auf der Seite derer, die im Nachteil sind. Schon vor Ylvas Geburt beteiligte sie sich an der Quartiersarbeit im Viertel, gab Töpferkurse an der Schule, in die jetzt ihre Tochter geht. Dass so viele Menschen in Neukölln von Sozialhilfe leben müssen, bedauert sie, schließlich könnten sie aufgrund ihrer fehlenden Ausbildung keinen Job finden. Dass manche das auch gar nicht wollen, das mag Lafrenz sich nicht so recht vorstellen. Und wenn schon: „Deren Kinder muss man mitziehen, damit sie später auf eigenen Füßen stehen!“ Mitziehen, das sagt Lafrenz gerne.

Ihre Kiezschulen-Initiative setzt sich dafür ein, dass bald auch die sogenannten bildungsnahen Eltern ihre Kinder im Viertel einschulen, anstatt sie in andere Bezirke zu fahren oder gar wegzuziehen. „Viele Eltern gucken sich die Schulen in ihrem Viertel nicht mal an." Lafrenz wirbt deshalb für die Schule vor ihrer Haustür. An den Tagen der offenen Tür verteilt sie dort Flyer, genauso wie in Kitas und Bars. Einmal im Monat gibt es ein Info-Treffen für interessierte Eltern, nicht immer kommt jemand. Aber davon lässt sich Lafrenz nicht aufhalten, sie spricht auch mal Eltern mit Kind auf der Straße an, sie hat jetzt eine Mission.

In der Karl-Weise-Schule wird jahrgangsübergreifend gelernt, Erzieher unterstützen die Lehrer bei der Betreuung, es gibt AGs, in denen die Schüler kochen, gärtnern oder Theater spielen. Die Schule beteiligt sich am Kiezfest, um 
ihre Arbeit vorzustellen, interessierte Eltern können mit ihren Kindern stundenweise am Unterricht teilnehmen. Bisher vergeblich. Es gebe noch keinen Zuwachs bei den Anmeldungen deutscher Eltern, sagt Andrea Schwenn, die Leiterin der Karl-Weise-Schule. „Aber zum Tag der offenen Tür kommen mittlerweile schon mehr deutsche Eltern als früher.“

Petra Lafrenz hat den Schulbeginn ihrer Tochter sorgfältig vorbereitet. Sie hospitierte mit Ylva im Unterricht, damit sich die Tochter schon einmal an die Schule gewöhnen konnte. Eine friedliche Yogastunde hat Lafrenz damals im Bewegungsraum der Schule erlebt. 20 Schüler hockten im Schneidersitz auf blauen Yogamatten und hielten ihre Hände vor der Brust zusammen. „Herzlich willkommen“, sagte die Lehrerin, „Namaste“. Nichts erinnerte an das Angstbild einer Brennpunktschule, in der sich die Schüler gegenseitig beschimpfen und verprügeln. Nur im Klassenzimmer nebenan wurde es nach kurzer Zeit laut: „Geh auf deinen Platz!“, schrie ein Lehrer gut hörbar durch die Wand in die Entspannungsübungen. „Auf deinen Platz!“

Die Liste der Probleme, die es an Brennpunktschulen gibt und vor denen Eltern sich fürchten, ist lang. Für manch eine Mutter ist es schon schwer genug auszuhalten, wenn ihr sechsjähriges Kind mittags nach Hause kommt und „du Hure“ sagt. Wenn aber der Unterricht nicht stattfinden kann, weil Kinder kein Wort Deutsch sprechen, wenn Kinder im Unterricht überhaupt nicht mehr mitkommen, weil sich zu Hause niemand um ihre Hausaufgaben kümmert oder darum, wann sie ins Bett gehen, wenn Kinder aggressiv oder gar gewalttätig sind, weil sie es nicht anders kennen aus ihrer Familie, dann ist die Schmerzgrenze besorgter Eltern meistens erreicht - und der klassische Konflikt perfekt. Denn natürlich sind sich gerade die aufgeklärten Akademikereltern darüber im Klaren, dass die Problemkinder nichts für das Umfeld können, aus dem sie kommen, und natürlich ist es richtig und hört sich gut an, wenn Petra Lafrenz sagt, dass man gerade solche Kinder mitziehen müsse. Weil sie noch schlechtere Bildungschancen haben würden, wenn sämtliche deutschen Eltern ihre Kinder an sorgfältig ausgewählten Schulen einschulen ließen. Davor warnen nicht nur Politiker wie Buschkowsky, sondern auch Bildungsexperten, die in Vierteln wie Neukölln längst von Segregation sprechen, einer Entmischung. Sie sehen die Eltern in der Pflicht, dafür zu sorgen, ein noch stärkeres Auseinanderdriften der Gesellschaft zu stoppen.

Es ist eine jener Debatten, in denen es leichtfällt, auf der richtigen Seite zu stehen - solange man nicht selber betroffen ist.

Lilia Kleemann, die ihren Sohn außerhalb eingeschult hat, kann Kritik an ihrer Entscheidung zwar verstehen. „Aber ich finde es aufgesetzt, zu sagen, dass ich mein Kind extra in eine Brennpunktschule schicke, damit sich dort etwas ändert.“ Sie wolle nun einmal das Beste für ihr Kind. „Und dazu gehört 
das räumliche und soziale Umfeld, in dem es lernt.“ Auch Kleemann weiß, dass das Beste fürs Kind nicht unbedingt das Beste für die Gesellschaft ist. Aber muss Integration über das eigene Kind stattfinden? „Ich denke nicht“, sagt Kleemann. „Ich glaube, es ist sinnvoller, als Erwachsener in die Politik zu gehen, als das Kind zu benutzen, um Schulen stärker zu durchmischen.“

Sie habe sich vor einem Jahr viele Schulen in Neukölln angesehen. Als das Amt den Bescheid für die Einzugsschule schickte, war klar, dass sie Liou nicht dorthin schicken würde. Ihr hatte die Stimmung an der Schule nicht gefallen. „Ich denke, jeder sollte für sein Kind die richtige Schule suchen“, sagt sie. Dass Behörden darüber entscheiden, wo ihr Kind lernt, hält Kleemann für falsch. „Die Schulen sollten frei wählbar sein.“ Wäre es so, glaubt sie, würden sich die Schulen vielleicht auch mehr anstrengen, ein Angebot zu schaffen, das Eltern wirklich überzeugt.

Beim Thema Schulflucht geht es längst um mehr als um die Angst deutscher Eltern vor der Brennpunktschule. Es geht um die Ansprüche einer neuen Generation von Müttern und Vätern, die so aufgewachsen sind, dass sie stets frei wählen konnten, egal, ob Partner, Beruf oder die Stadt, in der sie leben wollen. Mit den starren Strukturen des deutschen Bildungssystems haben sie gleichermaßen Probleme wie mit den Zuweisungen ihrer Kinder auf bestimmte Schulen in Wohnortnähe.

Es habe eine Schule in ihrem Viertel gegeben, auf die sie Liou sofort geschickt hätte, sagt Lilia Kleemann. Die habe auch ein schwieriges Einzugsgebiet. Vor allem habe sie aber ein Konzept, das sie überzeugt habe. Kleemann bewarb sich und versuchte alles, damit ihr Sohn dort hingehen könnte. Es hat aber nicht geklappt, die Schule war völlig überlaufen. Eltern lassen sich also durchaus überzeugen vom Konzept einer Schule, obwohl sie mitten im sozialen Brennpunkt liegt. Auch deshalb fordern die Eltern: Schafft mehr gute Angebote! Beeindruckt uns! Was sich mit mehr Engagement bewirken lässt, zeigt die Entwicklung der Rütli-Schule. Die Problemschule, deren Lehrer damals um Hilfe riefen, ist mit mehr Geld und Personal inzwischen quasi zu einer Vorzeigeschule geworden. Probleme mit mangelnden Anmeldungen deutscher Schüler gibt es dort jedenfalls nicht mehr.

Vom Einsatz der Schulleiter und Lehrer hänge eine Menge ab, sagt Petra Lafrenz. Die Leiterin der Karl-Weise-Schule sei unheimlich engagiert. Lafrenz gibt zu, dass sie auch ihretwegen glaube, dass ihre Tochter auf der Schule gut aufgehoben sei. Ob es ihre Initiative schafft, mehr deutsche Eltern dazu zu bewegen, ihre Kinder in Neukölln einschulen zu lassen, bleibt abzuwarten. Mittlerweile hat sie 40 Unterstützer. Aber die Kinder der meisten sind noch zu klein, um in die Schule zu gehen. Im Moment ist es also ein sehr theoretisches Gegenprogramm zur Schulflucht - selbst wenn es der Initiative gelänge, mehr 
deutsche Eltern davon zu überzeugen, sich die Schulen in ihrem Viertel zumindest mal anzusehen. Lafrenz glaubt, dass sich das Konzept und die Angebote einer Schule letztendlich auch durch die Anmeldezahlen deutscher Eltern verändern werden.

Bei all der Hysterie um das Segregationsverhalten deutscher Eltern wird schnell vergessen, dass es auch Familien ohne deutschen Pass gibt, die ganz ähnliche Vorstellungen von guten Schulen haben wie die Deutschen. „Ich habe überlegt umzuziehen“, sagt die Georgierin Tea Bliadze, deren Tochter Anna Maria in die Karl-Weise-Schule geht, in die Ylva gerade eingeschult wurde. Bliadze hörte die üblichen Geschichten von Neuköllner Schulen, von Prügeleien und Kindern, die kein Wort Deutsch sprächen. „Ich dachte, dass ich eine schlechte Mutter bin, wenn ich mein Kind hier zur Schule schicke.“

Vor sechs Jahren kam die 32-Jährige nach Deutschland und lebt seitdem in Neukölln. Weil ihr georgischer Studienabschluss in Deutschland nicht anerkannt ist, arbeitet die alleinerziehende Mutter als Altenpflegerin. Sie nimmt das hin und beschwert sich nicht. Auch nicht darüber, dass sie jeden Morgen um sechs Uhr anfangen muss zu arbeiten. Vor allem deshalb blieb ihr gar keine andere Wahl, als Anna Maria auf die Schule im Viertel zu schicken. „Wenn es nicht gegangen wäre, hätte ich nach einem halben Jahr neu überlegt“, sagt Bliadze. Aber ihrer Tochter gefällt es bestens. Ein Mädchen wie sie ist ein schöner Beweis dafür, dass ein hoher Anteil an Kindern mit Migrationshintergrund nicht automatisch ein Grund zum Fürchten sein muss. Anna Marias Muttersprache ist Deutsch, und auf die Frage, was ihr in der Schule am besten gefalle, sagt sie: „alles“. Wer in ihrer Klasse das Problemkind ist, weiß man nach zehn Minuten Unterricht. Es ist eines der wenigen deutschen Kinder. 
